\author{
MÉMOIRE \\ PRÉSENTÉ À \\ L'UNIVERSITÉ DU QUÉBEC À CHICOUTIMI \\ COMME EXIGENCE PARTIELLE \\ DE LA MAÎTRISE EN ÉDUCATION (M.A.)
}

\author{
PAR \\ JOSIANE GAGNON \\ BACHELIÈRE EN SCIENCE DE LA NUTRITION (B.Sc.)
}

Introduction d'une séquence d'aventure au programme de formation de l'école québécoise comme outil d'éducation à la santé, au troisième cycle du primaire 


\section{AVANT-PROPOS}

Le système d'éducation québécois, tout comme le système de santé, vit présentement plus d'une réforme. Bien que le renouveau pédagogique possède un potentiel de réussite, car il vise une formation globale, diversifiée et ouverte sur le monde, il connaît aussi plusieurs ratées. À ce jour, l'actualité trace un portrait plus qu'inquiétant de la santé des jeunes. L'activité virtuelle aurait pris la place de l'activité physique, et la soif de sensations se manifeste, particulièrement chez les jeunes, de façon de plus en plus malsaine (drogues, piercing, hypersexualisation...).

L'Homme qui utilisait ses sens pour survivre et se reproduire a vu sa machine humaine se dérégler, à vitesse Grand $V$ au cours des dernières décennies. Le plaisir, le goût de vivre et de bien se sentir s'actualisent de façon barbare, notamment par l'utilisation de succédanés ou l'adoption de comportements déviants. Mais où se cache le plaisir d'accomplir? Dans l'action? Dans l'effort corporel? Dans la réalisation? 
L'être que nous sommes vient et vit de la nature. La terre répond à l'ensemble de nos besoins de base et, pour s'émanciper, tout être humain doit éprouver des sentiments positifs qui favorisent la consolidation de son estime personnelle: sentiments d'identité, sentiments de sécurité, sentiments d'appartenance, mais surtout il doit se sentir compétent et que sa compétence doit être reconnue.

Les jeunes sont en développement. Leur corps est leur premier outil et il change... Le connaître, théoriquement parlant, est une chose, mais l'utiliser à bon escient en est une autre qui, à notre avis, est beaucoup plus significative et formatrice, car son activité génère, à court, moyen et long terme des sensations et du plaisir. Vivre l'expérience éducative permet l'émancipation du jeune et favorise son bien-être; en ce sens, nous croyons que l'éducation par l'aventure pourrait devenir un outil de choix pour le milieu scolaire.

L'aventure, c'est la découverte de soi, des autres et de son environnement. L'éducation permet de comprendre les phénomènes afin de mieux gérer l'aventure de la vie.

De la Hongrie, au Pérou en passant par le Nunavik, ce mémoire est empreint d'expériences vécues, d'aventures et de réflexions liées à l'émancipation de l'être humain. 
Nous posons ainsi un regard sur 1'aventure en milieu scolaire et traçons un portrait quant aux impacts probables sur le développement du jeune. L'introduction planifiée d'ateliers éducatifs par l'aventure en plein air, dans le Programme de formation de l'école québécoise, contribue à épicer les apprentissages de plaisir. Ce plaisir générerait la réussite et contribuerait, à notre avis, à améliorer la santé de la génération montante.

Je tiens à remercier tout spécialement ma directrice de maîtrise Diane Gauthier, professeure au Département des sciences de l'éducation de l'Université du Québec à Chicoutimi, qui a su me soutenir à travers les épreuves de ma vie, ainsi que dans la réalisation de ce mémoire. Par ses précieux conseils, son ouverture relativement à ma façon de voir la vie, ses encouragements, sa patience ainsi que ses interventions clés lors des moments de découragement. Merci! À mon codirecteur, Mario Bilodeau, professeur au Département de tourisme et d'aventure en plein air de 1'Université du Québec à Chicoutimi qui m'a inspirée et qui, avec un brin d'humour, a su nuancer mes envolées tout au long de ce travail truffé de joies, de doutes, d'espoirs, de certitudes et d'obstacles. Merci, à vous deux, pour vos commentaires, votre temps et vos nombreux conseils qui ont permis d'établir un pont entre l'intervention et la rédaction. 
Merci à la direction de l'école pilote ainsi qu'aux deux enseignantes collaboratrices (et à leur suppléante), dont je dois traire les noms afin de respecter les normes éthiques en vigueur dans la présentation de ce présent mémoire. Je les remercie d'avoir accepté de sortir de leur zone de confort en jumelant l'aventure plein air au renouveau pédagogique, mais surtout d'avoir participé étroitement à la réalisation de ce projet. Merci aux parents des élèves qui ont permis le bon déroulement en nous confiant leur progéniture malgré les incertitudes. Merci aussi aux membres de la communauté qui m'ont apporté leur soutien et leur expertise.

J'aimerais aussi mentionner qu'au fil de cette longue expédition, tous les gens que j'ai côtoyés ont laissé une empreinte dans mon carnet de route : les jeunes, mes collègues de travail et d'étude, mes frères, mes amis et connaissances... Merci à ma mère et à Jocelyne pour leur disponibilité et leur dévouement, à Bianka pour la touche finale.

Finalement, merci à mon compagnon de vie pour ses nombreux encouragements, sa patience et sa compréhension suivant principalement les moments de tempêtes. Merci à mes deux petits voyageurs de me permettre de vivre des moments exceptionnels jour après jour. Jèkim, Benédict et Nanouk, je vous dédie tout ce travail, pour vos rires, votre joie de vivre et surtout votre esprit d'AVENTURE!

Bonne lecture! 


\section{RÉSUMÉ}

La présente étude est inspirée des philosophies propres à l'éducation à la santé (Godin, 2002; Tones \& Green, 2004) à l'éducation en plein air (Neill \& Richard, 1998; Ford, 1986; Bilodeau, 1980), à l'éducation par l'aventure (Rabiola \& O'Keefe, 1999; Horwood, 1999; Cheryl \& Lupton, 1999) et à l'apprentissage expérientiel en plein air (Kraft, 1999). L'éducation par l'aventure en plein air est à notre avis un outil de choix qui offre aux jeunes l'occasion de se percevoir comme ils sont, des êtres uniques, indispensables les uns aux autres, évoluant dans un environnement commun : la nature.

Le défi réside dans la construction d'une intervention efficace en matière de promotion de la santé qui propose aux participants un climat propice aux apprentissages et des pistes de solutions pour favoriser leur bien-être et maintenir leur santé.

Les interventions vécues lors des ateliers éducatifs par l'aventure ont été adaptées au programme de formation de l'école québécoise, avec la collaboration des titulaires de classe. L'approche utilisée est globale et tient compte du développement d'habiletés sociales et psychomotrices, ainsi que de l'acquisition de connaissances de soi et de son environnement (Tones \& Green, 2004).

La conception d'une stratégie d'intervention active en promotion de la santé, au cœur du système scolaire, produit plusieurs connaissances. Les instruments utilisés pour la cueillette de données ont été multiples : observations actives et réflexives, fiches de retour avec question d'objectivation, questionnaires à l'intention de la direction et des parents et entrevues semi-dirigées avec les intervenants scolaires ${ }^{1}$. L'analyse effectuée est de type qualitative/interprétative (Savoie-Zajc, 2001) en lien avec le domaine général de formation Santé et bien-être et les compétences transversales d'ordre personnel et social ( 7 et 8 ). Pour faciliter 1'orchestration de 1'analyse, le vocable utilisé dans les documents émis par le comité École en santé (Martin \& Arcand, 2005) est observé. La dernière partie de l'analyse

\footnotetext{
${ }^{1}$ Pour l'ensemble du document, l'appellation « intervenants scolaires » inclut tous les acteurs du milieu scolaire ayant participé de près ou de loin à la réalisation du projet.
} 
pose un regard sur la transversalité et la pluridisciplinarité, autrement dit sur l'inclusion de ce mode d'intervention en milieu scolaire au cœur du programme de formation de l'école québécoise quant à son potentiel d'implantation.

Les principaux résultats dévoilent le développement d'une conscientisation en matière de santé, la manifestation positive de l'estime de soi chez les jeunes, ainsi que le potentiel de la séquence d'aventure pour la pluridisciplinarité des apprentissages. Ce modèle d'intervention exige une planification et une collaboration de plusieurs acteurs pédagogiques. Cette situation, selon Manidi et Dafflon-Arvanitou (2000), constitue un aspect limitatif à l'implantation de la séquence d'aventure et d'apprentissage dans le milieu scolaire.

Les données recueillies ont permis de dégager un modèle graphique (schémas 5.6.1) qui expose l'évolution anticipée des compétences personnelles et sociales au cours de l'année scolaire. Ce modèle expose plusieurs possibilités pour induire chez les jeunes une démarche réflexive en matière de Santé et bien-être en créant des situations d'apprentissage authentiques qui respectent les assises du renouveau pédagogique (2001) et permet le développement des compétences personnelles et sociales (Arcand, Daigle, Lapointe, Moreau, \& Rodrigue, 1998; Martin \& Arcand, 2005) des jeunes.

La présente étude cible un rapprochement entre les milieux scolaires et la santé par des actions concrètes et concertées. L'éducation par l'aventure en plein air est un outil d'intérêt qui réunit les efforts de l'ensemble des acteurs scolaires tout en offrant aux jeunes l'occasion de se percevoir comme des êtres authentiques, indispensables les uns aux autres et évoluant tous dans un environnement commun, c'est-à-dire la nature. 


\section{TABLE DES MATIÈRES}

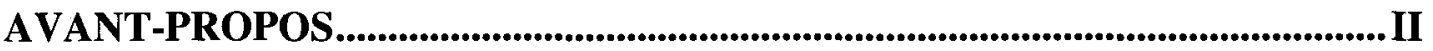

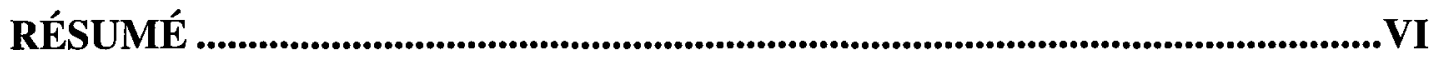

TABLE DES MATIÈRES............................................................................. VIII

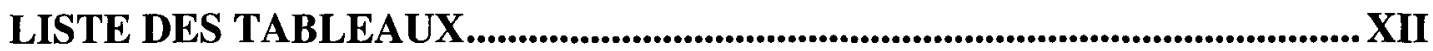

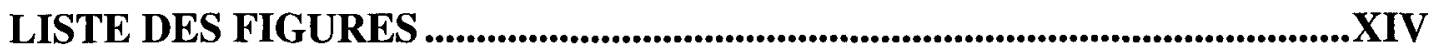

LISTE DES SCHÉMAS .............................................................................. XV

LISTE DES ACRONYMES......................................................................................................

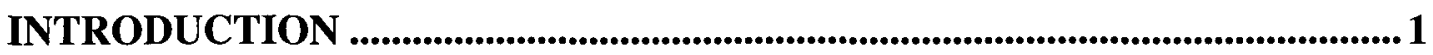

CHAPITRE 1 PROBLÉMATIQUE ................................................................4

1.1 Survol de la situation en matière de santé chez les jeunes ...........................5

1.1.1 Les comportements alimentaires et la pratique d'activité physique...... 7

1.1.2 La clientèle cible des programmes de santé............................................. 8

1.1.3 Un programme d'éducation à la santé selon les intérêts des jeunes .... 10

1.2 L'école comme lieu d'intervention en matière de santé ............................12

1.2.1 La santé à l'école... un brin d'histoire.................................................. 14

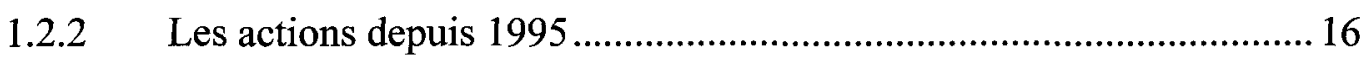

1.2.2.1 L'approche « École en santé »............................................................ 19

1.2.3 La place de la santé dans le virage vers le succès..................................20

1.3 Développer un mode d'intervention viable visant les axes de développement du DGF Santé et bien-être et le développement des compétences personnelles et sociales......................................................................................................224

1.3.1 L'estime de soi : facteur de protection en santé et en éducation ..........26

1.4 L'aventure éducative en plein air : un atout pour le MELS....................28

1.5 L'intérêt et l'originalité de cette recherche ..................................................30

1.6 Question de recherche et objectifs............................................................. 31

CHAPITRE 2 CADRE CONCEPTUEL ................................................................... 33

2.1 Définition de la santé ......................................................................................34 2.1.1 La préadolescence, une cible importante en matière de promotion de la santé 
2.1.2 Une vision de la santé et du bien-être selon Maslow.............................37

2.1.2.1 Un parallèle de la théorie de Maslow avec le DGF Santé et bien-être

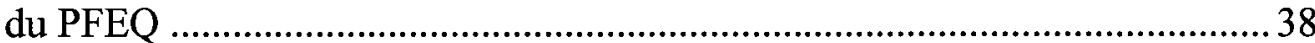

2.1.3 Une définition de la promotion de la santé en lien avec l'éducation à la

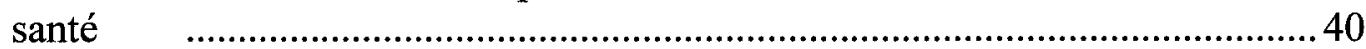

2.1.4 Les modèles et théories en matière d'éducation à la santé .................... 41

2.2 La santé à travers le renouveau pédagogique .............................................49

2.2.1 Le domaine du développement personnel du jeune............................. 51

2.2.2 L'éducation à la santé à partir du domaine général de formation Santé

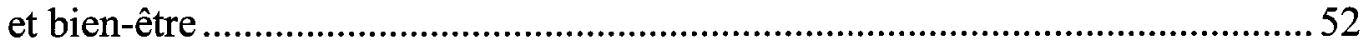

2.2.2.1 Rehausser l'estime de soi en renforçant les compétences

transversales d'ordre personnel et social ............................................................53

2.3 Les visées de l'estime de soi : définition....................................................55

2.3.1 La dimension physique du concept de soi et l'estime de soi ................57

2.3.2 Les compétences sociales (le soi social)..............................................59

2.3.3 Une stratégie d'intervention afin d'orienter positivement l'estime de soi du jeune et les compétences sociales ................................................................ 60

\subsection{L'éducation par l'aventure en plein air : un pas vers la santé! Les} retombées des modèles expérimentaux ......................................................................62 62

2.4.1 L'éducation en plein air (outdoor education) ........................................65

2.4.2 L'éducation par l'aventure (adventure education)...................................66

2.4.3 L'apprentissage expérientiel en plein air (experientiel learning) .........70

2.5 Les modèles expérimentaux en lien avec les théories de l'éducation .... 72 2.5.1 Un survol des liens existants entre l'aventure éducative en plein air et les théories de l'apprentissage ..............................................................................74

2.5.2 Intégrer la séquence d'aventure à la séquence d'apprentissage.............78

CHAPITRE 3 MÉTHODOLOGIE ................................................................83

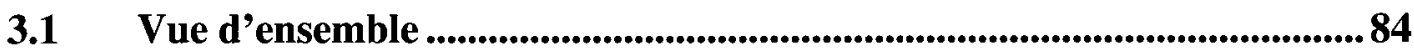

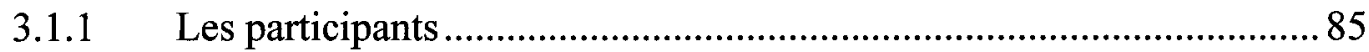

3.1.2 Mise en place de la recherche-action...................................................... 86

3.2 La planification du programme d'aventure éducative en milieu scolaire

3.2.1 La synthèse des ateliers selon le mode d'intervention adopté .................89

3.3 Les ateliers sous la loupe du PFEQ en lien avec l'éducation à la santé 92 3.3.1 Domaines d'apprentissage : éducation physique et à la santé, science et technologie, français............................................................................................ 95

3.4 Description de la séquence d'aventure et d'apprentissage en plein air vécue par les élèves du troisième cycle de l'école pilote...........................................97

3.4.1 Activités d'apprentissage ..................................................................98

3.4.2 Ateliers d'une demi-journée classe (A1 - A2 - A3 - A5 - A6 - A7 -

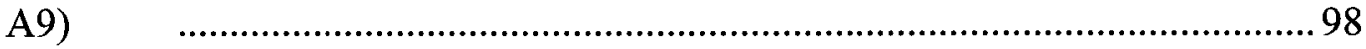

3.4.3 Excursions et expédition (A4 - A8 - A10)......................................... 99

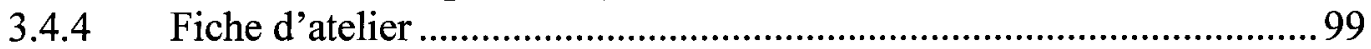


3.5 Les outils de collecte de données......................................................................... 100

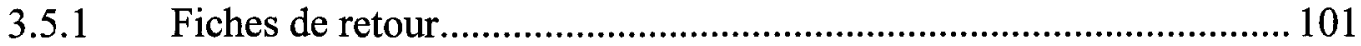

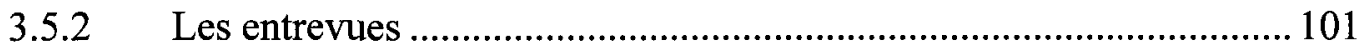

3.5.2.1 Enseignantes collaboratrices......................................................... 102

3.5.2.2 Enseignante d'éducation physique et à la santé............................... 102

3.5.3 Questionnaires à l'intention de la direction .......................................... 103

3.5.4 Questionnaires à l'intention des parents ............................................... 103

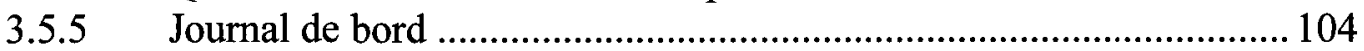

3.6 Déontologie ........................................................................................................... 105

3.6.1 Lettre de consentement destinée aux parents.................................... 105

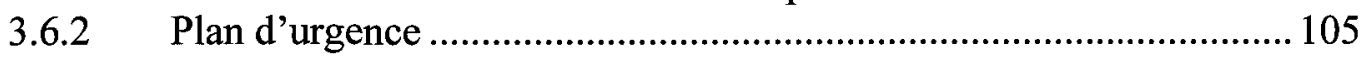

3.7 Analyse du contenu ............................................................................................ 106

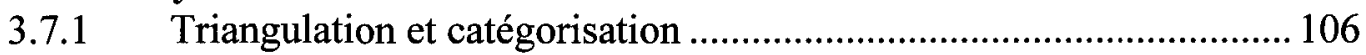

3.7.2 Examen phénoménologique et analyse thématique ............................ 112

CHAPITRE 4 RÉSULTATS ET ANALYSE ....................................................116

4.1 Les visées éducatives en matière de santé et de bien-être et les cibles atteintes pour chaque atelier ................................................................................................. 117

4.1.1 L'émergence de la connaissance en matière de Santé et bien-être

auprès des élèves .............................................................................................. 118

4.2 L'éducation à la santé en milieu scolaire favorisant le développement de

l'estime de soi ........................................................................................................... 123

4.2.1 Conscience corporelle..................................................................... 123

4.2.2 Modification des repères.................................................................... 126

4.2.3 Prise de conscience sur leur rapport avec la nature conduisant vers le

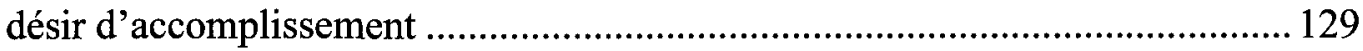

4.2.3.1 Manifestation de l'estime de soi ..................................................... 134

4.3 Structurer son identité et coopérer ............................................................... 137

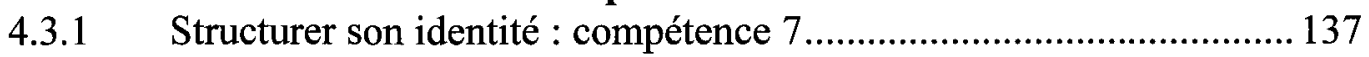

4.3.2 Coopérer : compétence 8 …………………….............................. 143

4.4 Habitudes de vie et comportements sécuritaires....................................149

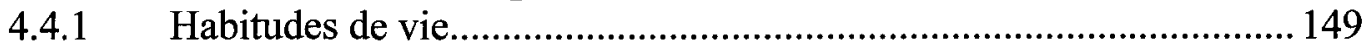

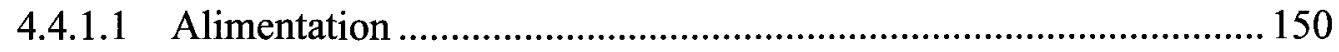

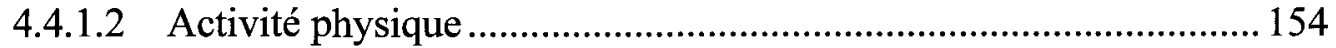

4.4.1.3 Bien-être psychologique et habileté à gérer son stress et ses émotions

4.4.2 Comportements sains et sécuritaires............................................... 158

4.4.2.1 Hygiène et sécurité ......................................................................... 158

4.5 Synthèse des résultats de la réalisation de la séquence d'aventure et les retombées à court et moyen terme................................................................................. 163

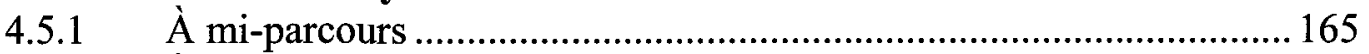

4.5.2 À la fin du projet.............................................................................. 165

4.5.3 Deux semaines après l'expédition finale, de la séquence d'aventure et

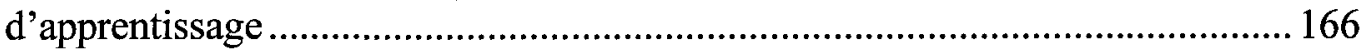


4.6 La transversalité et la pluridisciplinarité .................................................167

4.6.1 La séquence d'éducation par l'aventure : une pédagogie intuitive.... 170

4.7 Les commentaires généraux des différents adultes impliqués à la suite

de réalisation de la séquence ..................................................................................... 173

4.7.1 La direction de l'école ................................................................... 174

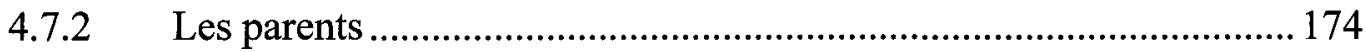

CHAPITRE 5 DISCUSSION................................................................................... 177

5.1 Une vision systémique de la séquence d'ateliers ...................................... 179

5.2 Les portées pédagogiques de la séquence d'aventure............................... 184

5.3 Un modèle d'éducation à la santé................................................................... 187

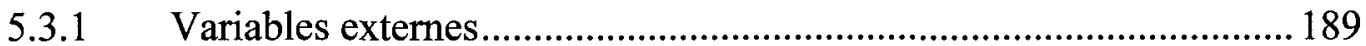

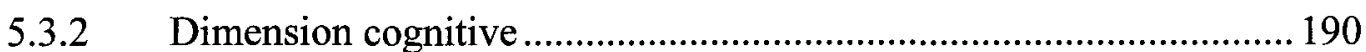

5.3.3 L'environnement naturel et son impact sur l'estime des jeunes......... 191

5.4 Compétences sociales et habiletés physiques.......................................... 193

5.4.1 Relations des élèves avec les différents intervenants impliqués......... 194

5.4.2 La relation avec les pairs .................................................................. 195

5.5 L'impact des ateliers sur les facteurs clés du développement du jeune en matière de Santé et bien-être ........................................................................................ 196

5.5.1 Comportements sains et sécuritaires en matière de santé dans la pratique d'activités de plein air

5.6 La séquence d'aventure, l'évolution des défis éducatifs : le modèle imaginé .......................................................................................................................... 198

5.7 Les pistes d'implantation ................................................................................ 202

5.8 Les obstacles à l'intervention................................................................203

5.9 Les forces et les limites de la recherche ....................................................... 205

5.10 Des pistes pour de futures recherches........................................................206

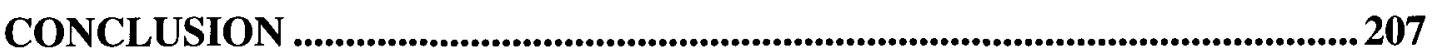

BIBLIOGRAPHIE......................................................................................................... 210

ANNEXES ..........................................................................................................................224 


\section{LISTE DES TABLEAUX}

Tableau 1.1.1 : Statistiques en matière de masse corporelle chez les jeunes .................. 6 Tableau 2.4.1 : Résumé des impacts perçus lors d'évaluations de modèles expérimentaux d'aventure éducative en plein air.

Tableau 3.2.1 : Ateliers d'aventure éducative en plein air suivis par les élèves du troisième cycle du primaire

Tableau 3.3.1 : Les sous-axes du domaine général de formation Santé et bien-être concernés par les ateliers d'éducation par l'aventure proposés dans le projet « Nature et Santé ».

Tableau 3.3.2 : Compétences transversales d'ordre personnel et social (7 et 8) concernées par les ateliers d'éducation par l'aventure proposés dans le projet « Nature et Santé ».

Tableau 3.3.3 : Domaines d'apprentissage et leurs composantes étroitement cernés par les ateliers d'éducation par l'aventure proposés dans le projet « Nature et Santé » 97

Tableau 3.3.4 : Exemple de relevé des thèmes pour le DGF Santé et bien-être 109

Tableau 3.3.5 : Exemple de relevé des thèmes pour la compétence transversale Structurer son identité

Tableau 3.3.6 : Exemple de relevé des thèmes la compétence transversale Coopérer.. 111

Tableau 4.1.1 : Intentions éducatives et cibles atteintes en matière de Santé et bien-être pour les ateliers 1 à 5

Tableau 4.1.2 : Intentions éducatives et cibles atteintes en matière de Santé et bien-être pour les ateliers 6 à 8

Tableau 4.1.3 : Intentions éducatives et cibles atteintes en matière de Santé et bien-être pour les ateliers 9 et 10

Tableau 4.2.1 : Besoins physiques

Tableau 4.2.2 : Besoin de sécurité

Tableau 4.2.3 : Besoin d'acceptation et d'épanouissement comme garçon ou fille...... 129

Tableau 4.2.4 : Besoin d'actualisation de soi

Tableau 4.2.5 : Estime de soi - Extraits du journal de bord de la chercheure-acteure et citations (ou écrits) des jeunes

Tableau 4.2.6: Facettes prédominantes des ateliers relativement à la conscience de soi et au développement de l'estime de soi . 
Tableau 4.3.1 : S'ouvrir aux stimulations environnantes : exemples et manifestations 139 Tableau 4.3.2 : Prendre conscience de sa place parmi les autres : manifestations et propos

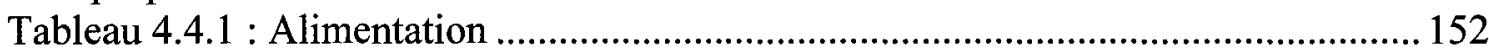

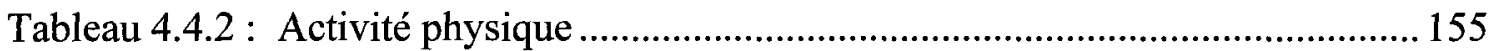

Tableau 4.4.3 : Relaxation, gestion du stress, des émotions..................................... 157

Tableau 4.4.4 : Hygiène et conduite sécuritaire en toutes circonstances...................... 160

Tableau 4.4.5 : Activité physique intégrée en classe, à l'école, dans la famille et dans d'autres milieux

Tableau 4.5.1: Synthèse des réponses recueillies à mi-parcours et au retour de l'expédition finale : «Est-ce que le projet Nature et santé leur a permis de découvrir (d'apprendre) de nouvelles choses à l'école?»

Tableau 5.2.1 : Éléments émergents des ateliers d'aventure et de plein air, réalisés par les jeunes du troisième cycle du primaire $\left(5^{\mathrm{e}}\right.$ et $6^{\mathrm{e}}$ année $)$ 186

École Fréchette de l'Anse-Saint-Jean. 


\section{LISTE DES FIGURES}

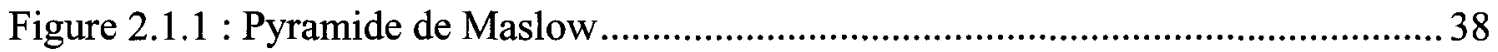

Figure 2.1.2 : Modèle intégrateur de Godin (2002) en lien avec les différents modèles et théories de l'éducation à la santé privilégiés en Amérique du Nord (Turcotte, 2006,

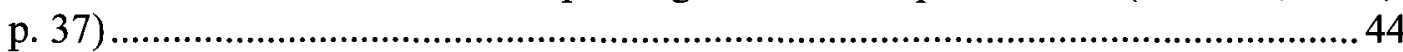

Figure 2.1.3 : Health Action Model- HAM. (Tones \& Green 2004) ............................... 48

Figure 2.4.1 : « Dépasser ses limites pour évoluer " ...................................................69 Figure 2.5.1 : Illustration de la structure d'une tâche en conformité avec la formation par

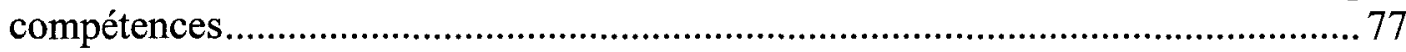

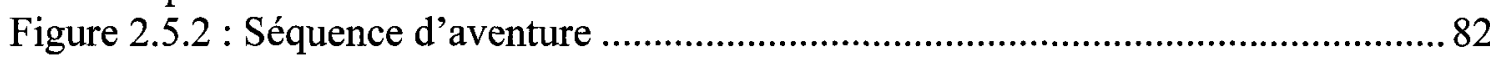

Figure 5.3.1 : Modèle intégrateur de Godin (2002) en lien avec les différents modèles et théories de l'éducation à la santé privilégiés en Amérique du Nord (Turcotte 2006) adapté selon les résultats d'analyse de la présente étude. 


\section{LISTE DES SCHÉMAS}

Schéma 1.2.1: Représentation schématique du Programme de formation de l'école québécoise (PFEQ, 2001) ................................................................................22

Schéma 2.1.1 : Dimensions de la santé (Naidoo \& Wills, 1994) .....................................35

Schéma 5.1.1 : Modèle descriptif de l'éducation par l'aventure et le plein air dans le PFEQ

Schéma 5.6.1 : L'évolution anticipée des compétences personnelles et sociales au cours de l'année scolaire, lors de la mise en place d'une séquence d'aventure et d'apprentissage adoptant une approche holistique d'éducation à la santé. 


\title{
LISTE DES ACRONYMES
}

\author{
AES L'approche École en santé \\ AQESSS Association québécoise d'établissements de santé et de services sociaux \\ CT Compétences transversales \\ CAAO Collectif action alternative en obésité \\ DD Domaines d'apprentissage \\ DGF Domaines généraux de formation \\ HAM Health Action Model / modèle de la santé par l'action \\ INSPQ Institut national de santé publique du Québec \\ MELS Ministère de l'Éducation, du Loisir et du Sport \\ MEQ Ministère de l'Éducation du Québec \\ MSSS Ministère de la Santé et des Services sociaux \\ OMS Organisation mondiale de la santé \\ PFEQ Programme de formation de l'école québécoise \\ SAE Situation d'apprentissage et d'évaluation \\ TCNPP Table de concertation nationale en promotion et en prévention
}




\section{INTRODUCTION}

En 1996, la Commission des États généraux sur l'éducation recommande de viser plus largement le développement et le plein épanouissement de la personne, ce qui se traduit notamment dans le PFEQ, par une plus grande place octroyée à la santé (MEQ, 1996). Dans ce renouveau pédagogique, les modèles pédagogiques changent, les stratégies d'enseignement se modifient et les enseignants sont désormais perçus comme des guides favorisant le cheminement des apprenants. Chaque établissement d'enseignement, tout en respectant un cadre prédéterminé dans le PFEQ, établit maintenant ses orientations de même que les moyens pour les atteindre (PFEQ, 2001). Désormais, tel que le mentionne littéralement le PFEQ : « cette responsabilité déborde largement le cadre des seules interventions propres au programme d'éducation physique et à la santé; elle requiert une action concertée de l'ensemble du personnel " (PFEQ, 2001, p. 44). Chaque milieu scolaire cherche des façons novatrices de favoriser l'épanouissement global des jeunes à l'école.

En 1997, dans un article intitulé Adventure Education and Outward Bound: Out-of-Class Experiences that Make a Lasting Différence, Hattie, Marsh, Neill et Richard, rapportent que les expériences en plein air développent la santé corporelle 
et soutiennent la santé de l'esprit. Ainsi, l'éducation par l'aventure et le plein air revêtirait plusieurs atouts pour améliorer la santé et la réussite scolaire des jeunes.

En 2001, l'Enquête sociale et de santé auprès des enfants et des adolescents québécois 1999 rend publics des constats inquiétants en matière de santé chez les jeunes. Outre la problématique reliée à l'usage du tabac, de l'alcool et de la drogue, le taux de suicide, de détresse psychologique, l'obésité et la préoccupation à l'égard de l'image corporelle sont en hausse chez les jeunes. Aussi, la même enquête note que le nombre d'heures consacrées à la pratique d'activités physiques chez les jeunes est en dégringolade et ce, tant chez les garçons que chez les filles.

Ainsi en 2003, le ministère de la Santé et des Services sociaux (MSSS) et le ministère de l'Éducation du Québec (MEQ) s'allient officiellement, en créant l'approche École en santé, afin de favoriser l'épanouissement d'une génération montante éduquée et en santé. Dans le but de soutenir cette action, nous croyons fermement que l'aventure éducative en plein air, riche en potentiel tant du côté de la santé que de l'éducation, aurait sa place au cœur du Programme de formation de l'école québécoise (PFEQ).

Dans l'optique du renouveau pédagogique, nous considérons le milieu scolaire comme un endroit idéal pour construire des références durables en matière de santé. Développer chez les jeunes le goût et l'habitude de faire de l'activité physique (Thibault, 2001), de bien s'alimenter et d'adopter des comportements sains et 
sécuritaires en matière de santé exige, à notre avis, un mode d'intervention actif tenant compte de l'intérêt des jeunes.

À titre d'exemple, l'éducation par l'aventure en plein air semble réputée pour rehausser l'estime et le concept de soi des jeunes participant à ce type d'activité (Gass, 1993; Neill, 2000; Neill \& Richard, 1998; Ann Klint, 1999). Ce facteur de protection, en matière de santé, est inscrit comme un «apprentissage commun » du domaine du développement personnel du PFEQ (2001) et fait partie des facteurs clés à considérer pour améliorer la santé des populations (Martin \& Arcand, 2005), tout comme le développement de saines habitudes de vie. De Koninck (2004) explique qu'une habitude se déploie au fil d'exercices et se consolide par l'acquisition de connaissances. Ainsi, le milieu scolaire est un milieu privilégié pour introduire une éducation à la santé qui utilise des stratégies d'apprentissage actives, développant ainsi de saines habitudes de vie.

Dans la présente étude, nous tenterons de démontrer la faisabilité d'un tel mode d'intervention en milieu scolaire et nous identifierons les retombées potentiellement positives sur la santé et la réussite des jeunes qui auront participé à l'ensemble de la séquence d'aventure et d'apprentissage. L'école a aujourd'hui une mission de prévention et de promotion en matière de santé (Martin \& Arcand, 2005); le présent mémoire présente donc une stratégie d'intervention novatrice qui actualise les pratiques d'éducation à la santé en milieu scolaire, tout en favorisant la réussite scolaire de cette génération montante. 


\section{CHAPITRE 1}

\section{Problématique}

Ce chapitre trace, dans un premier temps, un portrait de la situation en matière de santé chez les jeunes, pose un regard historique sur la place de la santé en milieu scolaire, puis identifie la clientèle cible ainsi que l'intérêt des jeunes en matière de pratique d'activité physique. Dans un deuxième temps, ce chapitre met en lumière les conditions favorables à la mise en place d'un programme d'éducation à la santé dans le système scolaire, ainsi que les angles d'action à définir pour actualiser des situations d'apprentissage et d'évaluation $(S A E)$ et permettre à l'ensemble de l'équipe-école de participer au développement de compétences favorisant la santé et le bien-être (MEQ, 2003) des jeunes. 


\subsection{Survol de la situation en matière de santé chez les jeunes}

Les huitièmes Journées annuelles de santé publique, tenues à Montréal à l'automne 2004 (Institut national de santé publique du Québec, 2004), ont révélé que le Québec est marqué par une prévalence croissante des problèmes de santé chez les jeunes : obésité embonpoint, sédentarisation, alimentation inadéquate, diabète de type 2, hypertension et maladie cardiovasculaire pour ne nommer que ceux-là. Ces problèmes engendrent une faible estime de soi conduisant à des difficultés d'apprentissage et d'adaptation sociale (Hamel, 2001; Duclos, 2000). De Koninck (2004) croit que notre civilisation est en perte grave de valeur tactile et ce, principalement dans l'éducation de nos enfants. Cette privation de sensations tactile, gustative, olfactive et auditive a selon lui des effets sur le développement psychique des enfants.

L'enquête québécoise d'envergure menée en 1999 auprès de 3700 enfants et adolescents de 9,13 et 16 ans, sous la direction de Jacinthe Aubin, révèle des chiffres inquiétants. Déjà à l'âge de 13 ans, $11 \%$ des jeunes consomment à l'occasion une grande quantité d'alcool soit cinq verres ou plus (Morissette, Dedobbeleer \& Rojas Viger, 2002). Par ailleurs, la prévalence de fumeurs actuels se chiffre à $5 \%$, chez les élèves de première année du secondaire. Dans un autre ordre d'idées, des études concernant la prévalence à l'égard de l'obésité démontrent que, dans tous les pays du monde, l'obésité infantile augmente sans cesse (Caflish \& 
Paris, 2001; Schaefer \& Mongeau, 2000). L'Enquête sociale sur la santé des enfants et des adolescents québécois 1999 (Tableau 1.1.1) porte un nouveau regard et mentionne qu'il y a actuellement un double problème à l'égard du poids chez les jeunes. D'une part, la prévalence de l'obésité se situe à $3 \%$ ou $4 \%$, selon l'âge, et autour de $11 \%$ à $13 \%$ pour l'embonpoint; d'autre part, le taux d'insatisfaction à l'égard de l'image corporelle est de $45 \%$ chez les jeunes de 9 ans et d'environ $60 \%$ chez ceux de 13 et 16 ans (Ledoux, Mongeau \& Rivard, 2002).

Tableau 1.1.1 : Statistiques en matière de masse corporelle chez les jeunes ${ }^{2}$

\begin{tabular}{|l|l|l|l|l|l|l|l|}
\hline \multirow{2}{*}{} & \multicolumn{2}{|c|}{9 ans } & \multicolumn{3}{c|}{13 ans } & \multicolumn{2}{c|}{16 ans $^{3}$} \\
\cline { 2 - 8 } & garçons & filles & garçons & filles & garçons & filles \\
\cline { 2 - 8 } & \multicolumn{3}{|c|}{$\%$} & \multicolumn{3}{|c|}{} \\
\hline Obésité & 4,2 & 3,5 & 3,2 & 3,5 & 3,3 & 3,2 \\
\hline Embonpoint & 10,9 & 11,1 & 11,8 & 11,2 & 12,2 & 12,6 \\
\hline Insatisfaction à l'égard de l'image corporelle & 43,7 & 45,5 & 59,9 & 56,6 & 58,6 & 61,4 \\
\hline
\end{tabular}

Par l'observation des comportements axés sur la modification de la masse corporelle, soit un gain ou une perte, ces auteurs remarquent que même si $80 \%$ des enfants de 9 ans présentent une masse corporelle normale, $45 \%$ d'entre eux s'en préoccupent. D'autre part, les statistiques démontrent qu'environ $8 \%$ des enfants de 9 ans rapportent avoir des idées suicidaires sérieuses (Breton, Légaré, Laverdure \& D’Amours, 2002) et dans ce dossier, depuis le début des années 1990, la moyenne des Québécois de 10 à 24 ans est supérieure à celle des jeunes Canadiens. Toujours en santé mentale, les chercheurs associent la détresse psychologique à une faible estime de soi. Breton et al. (2002) suggèrent notamment qu'une bonne estime de soi

\footnotetext{
${ }^{2}$ Ce tableau n'indique pas les coefficients de variation. Source : Ledoux et al., 2002.

${ }^{3}$ Exclut les jeunes de 16 ans qui ne fréquentent pas une école secondaire.
} 
constitue un facteur de protection contre la détresse psychologique, cette dernière étant étroitement en lien avec la consommation de substances psychoactives (alcool et drogues) et le suicide. L'estime de soi ou la perception positive de soi constitue l'un des facteurs clés afin de préserver une bonne santé globale.

\subsubsection{Les comportements alimentaires et la pratique d'activité physique}

Souvent perçus comme éléments d'ancrage en matière de santé, les comportements alimentaires et la pratique d'activité physique des jeunes préoccupent de plus en plus les responsables de la santé publique (Hamel, 2001; Barnett \& Hamel, 2002; Brassard \& Mongeau, 2004). Les auteurs de l'Enquête sociale sur la santé des enfants et des adolescents québécois 1999 rapportent que $20 \%$ des enfants de 9 ans ne mangent rien et ne boivent pas avant le début des activités (Bertrand, Nadeau, Stan \& Paquette, 2002) et ce, quotidiennement. Chez les enfants et les adolescents, ce comportement peut altérer des fonctions cognitives comme la mémoire, la capacité de traiter l'information et le processus d'apprentissage.

$\mathrm{Au}$ chapitre de la pratique d'activité physique, Thibault (2000) remarque qu'une baisse de l'activité physique se manifeste graduellement entre le début et la fin du secondaire. Barnett et Hamel (2002) révèlent que la majorité des enfants et des adolescents québécois n'ont pas une pratique d'activité suffisante et que la pratique des garçons est plus importante que celle des filles. Pourtant, la pratique régulière de 
l'activité physique dès l'enfance réduit le risque de maladies chroniques (Sallis \& Owen, 1999), diminue les symptômes associés à la dépression (Thibault, 2000) et est associée à une meilleure estime de soi en plus de favoriser aussi la réussite scolaire (Martin \& Arcand, 2005).

\subsubsection{La clientèle cible des programmes de santé}

Dans les programmes de prévention, les préadolescents sont une clientèle cible, notamment en ce qui a trait à la consommation et à l'abus d'alcools et de drogues (Morissette $e t$ al., 2002). D'autres problèmes de santé en émergence dans la société sont étroitement liés à la difficulté qu'ont les jeunes à traverser cette période de transition. Claes (1994) soutient que le jeune de 10 à 12 ans commence un processus progressif qui modifiera, à travers les expériences vécues, sa représentation de soi et d'autrui.

Chez les filles, l'augmentation de la quantité de tissus adipeux est l'un des facteurs déterminants de l'insatisfaction envers l'image corporelle. Brassard \& Mongeau (2001) remarquent que cette insatisfaction se traduit par des tentatives de perte de poids, alors que chez les garçons les comportements adoptés sont plutôt l'excès d'exercice et la consommation de produits ergogéniques. Harter (1990), Herry et Worth Gavin (1996) et Claes (1994) notent que vers l'âge de huit ans, la manifestation du concept de soi global s'amorce à la baisse, c'est-à-dire tend à

diminuer. À cet âge, l'enfant commence à se comparer aux autres, il utilise alors les 
réactions de ses pairs ainsi que leurs comportements pour ajuster son concept de soi, notamment sous la dimension sociale (Harter, 1990 dans Herry \& Worth Gavin, 1996).

D'autres auteurs (Hamel, 2001; Barnett \& Hamel, 2002; Breton et al., 2002 et Bernier \& Brochu, 2002) soulignent l'importance d'intervenir tôt et croient que, dans une perspective de prévention en santé, les efforts devraient être mis sur le développement de l'estime de soi au cours de l'enfance et dès le début de l'adolescence. Famose (2001) considère que l'amélioration de l'estime de soi, de l'image corporelle et des compétences sociales doit être vue comme un objectif fondamental en éducation. La préadolescence est donc une période déterminante car, l'insatisfaction à l'égard de l'image corporelle et la diminution de la pratique d'activité physique se manifestent (Brassard \& Mongeau, 2004; Thibault, 2000). Ces deux paramètres, ainsi que plusieurs comportements néfastes pour la santé, sont étroitement liés au degré d'estime de soi du jeune. Ouellet et Stan (2002) notent que, dès l'âge de 13 ans, les jeunes qui ont une estime de soi élevée aspirent, dans une proportion plus grande que ceux dont l'estime de soi est faible, à poursuivre des études postsecondaires.

Dans le même ordre d'idées, les conférenciers invités lors des huitièmes Journées annuelles de santé publique ont relevé la présence de liens entre l'adoption de saines habitudes de vie, l'estime de soi et les difficultés d'apprentissage et d'adaptation sociale (Ayotte, 1995; INSPQ, 2004). 
Pour inciter les enfants à adopter des comportements sains et sécuritaires en matière de Santé et bien-être, les stratégies pédagogiques utilisées doivent rejoindre leurs intérêts et favoriser le développement des compétences personnelles et sociales, ainsi que le rehaussement de l'estime de soi (Martin \& Arcand, 2005).

\subsubsection{Un programme d'éducation à la santé selon les intérêts des jeunes}

Actuellement, il existe peu de moyens de promouvoir une santé globale chez les jeunes (INSPQ, 2009). Les acteurs œuvrant dans les secteurs de la santé et des services sociaux déploient des efforts pour instaurer des actions, des concepts et des programmes de promotion et d'éducation en santé auprès des jeunes. Tel que le souligne Bastien (2001), ces actions sont souvent dispersées et incongrues, et ont peu de retombées bénéfiques pour les élèves. Il faut donc trouver un moyen de consolider les efforts de l'ensemble des acteurs du milieu scolaire que ce soient ceux du concierge, du travailleur social, des enseignants ou de la direction.

De 1995 à 2000, pendant que le ministère de l'Éducation réforme le système scolaire, plusieurs enquêtes sont menées afin de mieux comprendre la place de l'activité physique chez les jeunes. Le rapport, Les filles, c'est pas pareill', publié par Kino-Québec (Dugual, 1999), rapporte que dès l'âge de 11 ans, les filles sont moins nombreuses à être actives. L'auteure de cette enquête note que tout comme les garçons, les filles font de l'activité physique pour le plaisir et pour la camaraderie. Généralement, les jeunes sont d'accord avec la mixité des cours d'éducation 
physique, cependant les filles préfèrent les activités de type participatif axées sur le plaisir (Dugual, 1999) telles que les activités collectives, expressives, de plein air et individuelles. Une autre étude effectuée dans la région des Laurentides (Lapointe, Dubois \& Lapointe 2000), concernant la pratique de l'activité physique chez les jeunes de sixième année du primaire et de première année du secondaire, mentionne que parmi une liste de 23 activités, les jeunes s'intéressent davantage à des activités organisées telles que l'escalade, la planche à neige, le canot-camping, la randonnée pédestre, la randonnée à vélo et le soccer. Les auteurs de l'étude se sont aussi intéressés aux lieux où les jeunes préfèrent pratiquer des activités physiques : le plein air, les centres sportifs et la maison sont les lieux que les jeunes privilégient, tandis que l'école et le parc se classent bons derniers.

Barnett et Hamel (2002) soutiennent que la participation au cours d'éducation physique diminue avec l'âge. Aussi, selon l'étude de Beunard (1999), l'opinion des jeunes à l'égard du cours d'éducation physique serait en chute libre. Pour renforcer la participation des jeunes au cours d'éducation physique, le rapport de Kino-Québec réalisé par Dugual (1999) suggère une alternance de mixité et de non-mixité dans la classe d'éducation physique ainsi que la présence d'un enseignant dynamique, qui démontre les exercices et participe aux activités proposées.

Généralement responsable du développement de saines habitudes de vie, le cours d'éducation physique propose la plupart du temps des activités suivant une logique plus linéaire de la performance et de la compétition, tandis que les jeunes 
souhaitent une pratique axée sur la logique de type dilettante, privilégiant la découverte, l'émotion, le plaisir et la convivialité (Beunard, 1999; Dugual, 1999; Lapointe et $a l ., 2000)$. Déjà en 1933, Dewey, le père de l'apprentissage expérientiel, encourageait le questionnement du jeune par l'aventure et l'expérimentation (Jasper \& Wurdinger, 1999) mettant l'accent sur la découverte, l'émotion induite par la prise de risques et l'aspect social de l'apprentissage. Dugual (1999) et Lapointe et al. (2000) indiquent que les jeunes préfèrent un type d'activité physique en plein air qui leur offre l'occasion d'effectuer des apprentissages de groupe. Ainsi, tout comme le souligne Bastien (2001), les pratiques proposées en matière de santé dans les milieux sociaux, y compris l'école, sont à repenser.

Suzuki (2001) estime que la connaissance de l'environnement naturel pourrait grandement contribuer à l'amélioration de la santé et de la qualité de vie des jeunes. Si nous considérons les visées du PFEQ (2001) et les objectifs mis en place par la santé publique afin de promouvoir la santé des jeunes, l'éducation par l'aventure en plein air serait, à notre avis, un complément de choix.

\subsection{L'école comme lieu d'intervention en matière de santé}

Milieu privilégié, l'école possède plusieurs atouts permettant de mettre de l'avant des stratégies promotionnelles durables en santé, favorisant le plein développement du potentiel des individus. À cet égard, le groupe de travail interministériel (Arcand et al., 1998, p.1) sur les curriculums, volet Santé et bien-être 
a déclaré : «L'éducation contribue au maintien de la santé; la santé maintient les conditions nécessaires à l'apprentissage ».

Bien que la prévention et la promotion en matière de santé soient des phénomènes connus par les milieux sociaux tels que l'école, ils sont encore peu documentés. Bastien (2001) rapporte que deux dimensions en matière de promotion de la santé demeurent encore fortement négligées par la recherche sociale: la construction des savoirs préventifs en milieu scolaire ainsi que l'étude des interactions entre les acteurs pédagogiques (enseignants, infirmiers, psychoéducateurs). Pour développer des connaissances (Bastien, 2001, p. 3) et des pratiques en matière de santé, l'école représente une plateforme de recherche inestimable. Le milieu scolaire a pour mission depuis près d'un siècle d'offrir des savoirs préventifs et sanitaires en direction des élèves (Ball, 1993). L'Institut national de la santé et de la recherche médicale (2001), situé en France, ajoute que l'école est un lieu privilégié d'éducation et de promotion de la santé en raison des interactions importantes entre la santé et l'éducation, mais aussi parce qu'elle permet d'interpeller la quasi-totalité des enfants.

En ce sens, le rapport Brassard et Mongeau (2004) le démontre, l'école est un endroit propice pour l'insertion d'une multitude de programmes de prévention et de promotion de la santé. Elle permet de rejoindre une très grande proportion d'enfants et d'adolescents captivés et habitués à apprendre. Bastien (2001) et Brassard et 
Mongeau (2004) reconnaissent que l'école possède des attraits pour la planification de programmes en santé.

\subsubsection{La santé à l'école... un brin d'histoire}

Au Québec, il existe encore peu d'interventions efficaces en matière de promotion de la santé en milieu scolaire (INSQP, 2009). Pourtant, le système scolaire québécois en parle depuis le milieu des années 1960. Orientée vers une formation strictement scolaire au cours de cette décennie, l'école dispense aujourd'hui une formation dite intégrée. Considérée comme un milieu d'échanges et d'apprentissages, l'école a maintenant pour mission d'instruire, de socialiser et de qualifier (PFEQ, 2001) dans le but principal de préparer les jeunes à contribuer à l'essor d'une société voulue démocratique.

Pendant les années 1960, la Révolution tranquille amène une démocratisation

de l'éducation. À l'époque, l'enjeu est de faire entrer le Québec dans la modernité en éduquant sa population. En 1964, le rapport Parent énonce clairement que l'école : « de par sa structure, contraint les élèves à se tenir courbés sur leurs livres, sans leur accorder par ailleurs, l'occasion et le temps d'utiliser leurs facultés corporelles. Au lieu de la protéger et de la raffermir, comme elle devrait le faire, l'école elle-même porte atteinte à la santé des enfants » (Parent, Munroe, Filion, Lapointe, LaRoque, McIlhone, Rocher, De Rome \& Tremblay, 1964. p. 198). Dès lors, des actions sont 
prises en ce sens, un cours à saveur didactique s'installe (l'éducation physique) et le courant humaniste devient un élément important (Turcotte, 2006).

En 1979, un énoncé politique du gouvernement du Québec déclare que l'école québécoise doit viser à développer la personne dans toutes ses dimensions: physique, intellectuelle, affective, sociale, morale et spirituelle (École québécoise : énoncé de politique et plan d'action) et ce, au même titre que le recommandent les théoriciens en promotion de la santé (Naidoo \& Wills, 1994; Chamberland et al., 1996) par le modèle écologique.

En 1981, le ministère de l'Éducation juge que l'une des finalités de l'école québécoise est de développer un individu humaniste, c'est-à-dire une personne profondément enracinée dans son milieu et engagée dans la poursuite des valeurs humaines les plus élevées, mais aussi une personne à la recherche permanente de son bien-être physique et mental, consciente de ses propres valeurs et respectueuse de la dignité des autres (MEQ, 1981, p. 8). Pour y parvenir, le ministère de l'Éducation mise principalement sur le programme d'éducation physique. Ce cours considère l'élève comme un tout intégré. Ainsi, par ses actions, le jeune doit préserver la structure de son corps et établir des relations harmonieuses avec les différents environnements dans lesquels il évolue (MEQ, 1981). Bien que l'éducation physique partage les mêmes préoccupations que la physique (force, espace, temps), la chimie (échanges gazeux), les mathématiques (distance, trajectoire, forme), l'écologie (plein air) et qu'il existe une filiation intéressante avec d'autres domaines ayant pour thème 
central l'expression (MEQ, 1981), la cohésion des matières se heurte à un obstacle majeur de «type organisationnel ». C'est-à-dire que les liens entre les disciplines s'articulent difficilement; aussi, la préparation professionnelle (formations initiale et continue) et le manque de ressources matérielles semblent des obstacles. Turcotte (2006) soulève que le temps d'enseignement en santé semble toujours insuffisant et la collaboration entre les intervenants scolaires semble difficile.

\subsubsection{Les actions depuis 1995}

En 1995, les États généraux sur l'éducation ${ }^{4}$ ont pour but de faire le point sur l'état de la situation et de définir les orientations et les besoins du système d'éducation pour les prochaines années. L'école doit se recentrer sur sa mission. Le ministère de l'Éducation entreprend donc de revoir le système et rend publiques, en octobre 1996, les grandes orientations de la réforme qui s'intitule Prendre le virage du succès (MEQ, 1996). Dans le nouveau programme de formation, la santé du jeune est une priorité. Le PFEQ (2001) ouvre enfin une porte à la transversalité des disciplines par la mise en place de domaines généraux de formation (DGF) tels que Santé et bien-être.

En 1997, le ministère de la Santé et des Services sociaux rédige un document portant sur les priorités nationales de santé publique. Ce document recommande que, d'ici 2002, $60 \%$ des écoles disposent d'une programmation intégrée dans le

\footnotetext{
${ }^{4}$ Présidés par monsieur Paul Inchauspé. Source : MEQ (1996).
} 
domaine de la santé et des services sociaux visant le renforcement des aptitudes personnelles et sociales des jeunes et préconisant un environnement favorable à l'adoption de saines habitudes de vie (MSSS, 1997, tiré de Ouellet \& Stan, 2002). Or, à ce jour, la programmation intégrée dans ce domaine est à peine perceptible.

En 1999, lors de la Conférence des régies régionales de la Santé et des Services sociaux, le milieu scolaire est ciblé en tant que lieu d'intervention. Dans l'avis intitulé « Agir ensemble pour la santé et le bien-être des enfants et des adolescents », les auteurs formulent l'objectif suivant : « que les écoles offrent aux enfants et aux adolescents un milieu de vie favorable à leur développement et au maintien de leur santé et de leur bien-être » (Ouellet \& Stan, 2002, p. 109). Ceci rejoint les idéologies du PFEQ qui considère la santé et le bien-être comme central. Il mandate toute l'équipe-école pour y participer (PFEQ, 2001).

En 2004, lors des huitièmes Journées annuelles de la santé publique, trois ministères ${ }^{5}$ proposent d'agir en partenariat et lancent l'approche École en santé (AES), dans le but de contrer la prévalence croissante des problèmes liés à l'obésité et à l'embonpoint chez les jeunes. Les stratégies multiples élaborées dans l'approche proposent, entre autres, la formation d'une équipe multicommunautaire chargée de

\footnotetext{
${ }^{5}$ Collaboration interministérielle entre monsieur Alain Poirier, directeur national de santé publique et sous-ministre adjoint au ministère de la Santé, monsieur Robert Bisaillon, sous-ministre adjoint, Éducation préscolaire, enseignement primaire et secondaire au ministère de l'Éducation et monsieur Jean-Pierre Bastien, sous-ministre adjoint au ministère des Affaires municipales, du Sport et du Loisir. Préparé par un comité national d'orientation composé de représentant de l'Institut national de santé publique du Québec (INSPQ), du ministère de l'Éducation, du Loisir et du Sport (MELS) du ministère de la Santé et des Services sociaux(MSSS), de l'Association québécoise d'établissements de santé et de services sociaux et de la Table de concertation nationale en promotion et en prévention (TCNPP)
} 
mettre en place des actions visant à la fois les individus et leur environnement ((Promotion de la santé), 1986; INSPQ, 2004). L'approche École en santé est orientée selon un modèle hautement préconisé en matière de santé (Naidoo \& Wills, 1994, Chamberland et al., 1996), le modèle écologique, tout en cadrant avec les priorités du milieu scolaire.

S'articulant autour du développement humain, le concept École en santé met l'accent sur le développement de compétences, notamment l'habileté à réussir dans des contextes spécifiques (CAAO, 1997), en tenant compte de la société et de l'environnement. Le MSSS et le ministère de l'Éducation, du Loisir et du Sport (MELS) (Martin \& Arcand, 2005) partagent désormais un but commun, celui de rendre le jeune apte à prendre des décisions qui lui conviennent afin de maintenir sa santé, tout en respectant l'environnement dans lequel il évolue. À ce sujet, en 1938, Dewey mettait l'accent sur la richesse qu'apportent des apprentissages conjoints en salle de classe (Kraft, 1999) et au sommet d'une montagne par exemple. Ainsi, comme le mentionne Kraft (1999) en s'appuyant sur les propos de Dewey, la croissance du jeune sera physique, morale et non simplement intellectuelle : «(...) the active nature of all learning, that children learn best from concrete experience." (p.184). 


\subsubsection{L'approche «École en santé »}

À l'automne 2005, le gouvernement du Québec publie par l'intermédiaire du MELS un guide à l'intention du milieu scolaire et de ses partenaires : Pour la réussite éducative, la santé et le bien-être des jeunes (Martin \& Arcand, 2005). L'approche École en santé (AES) suggère de promouvoir la santé et le bien-être des enfants et des adolescents dans les écoles du Québec, notamment par la formation d'un comité composé, idéalement, de parents, de membres du personnel enseignant, de la direction de l'école et du personnel non enseignant, de représentants du conseil d'établissement ainsi que d'intervenants du CLSC et de la communauté dans l'école. L'AES prévoit aussi la mise en cuvre d'actions qui couvrent sept volets : les services de santé, les services sociaux, l'éducation à la santé, la formation et le soutien du personnel scolaire, le soutien aux parents, le soutien social aux enfants et l'environnement social, ainsi que l'environnement physique.

Bien que l'idée soit noble, les investissements demandés aux ressources du milieu pour la mise en place de ce type d'approche sont très énergivores (Bastien, 2001) puisqu'ils exigent la mobilisation de plusieurs acteurs. Chabot (2004) explique qu'une intervention directement au cœur du PFEQ (2001) doit être encadrée par un coordonnateur de projet qui assure la convergence des champs d'activité dans la direction des paramètres établis. L'annexe 1 résume le contexte de mise en œuvre de l'AES. 


\subsubsection{La place de la santé dans le virage vers le succès}

La littérature semble démontrer que l'une des plus grandes difficultés en éducation à la santé réside dans la coordination des matières disciplinaires entre elles (Bastien, 2001; Turcotte, 2006; Motta, 1998). À cet égard, Motta (1998) souligne clairement que l'un des obstacles d'intégration des pratiques d'intervention en éducation à la santé au contenu du curriculum scolaire réside dans la pratique des démarches traditionnelles des enseignants disciplinaires. Or, le PFEQ mise sur le développement de projets globaux et intégrateurs basés sur des sujets préoccupant les jeunes tels que la santé et le bien-être. Ces sujets dépassent amplement les frontières des savoirs disciplinaires. Toutefois pour bien développer les compétences menant au bien-être, l'acquisition de connaissances est essentielle (Tones \& Green, 2004).

Dans les pays occidentaux, la tendance actuelle serait de consolider l'inclusion de l'éducation à la santé en milieu scolaire puisqu'il semble clair qu'un individu en santé possède de meilleures aptitudes cognitives. Or, Manidi et Dafflon-Arvanitou (2000) voient une problématique importante dans le fait que la santé relève de plusieurs disciplines ou domaines d'enseignement puisque le mariage entre les enseignants et l'interdisciplinarité semble difficile (Turcotte, 2006). Ainsi, l'intégration d'une séquence d'activités d'aventure en milieu scolaire propose une occasion d'alliance avec les services de santé, tout en encourageant la collaboration entre intervenants scolaires. 
Le Programme de formation de l'école québécoise (PFEQ, 2001) s'inscrit dans la continuité de l'ancien programme et se caractérise par le développement des compétences, en portant une attention à la démarche pédagogique utilisée. Selon le PFEQ (2001), un parcours scolaire réussi doit faire en sorte que les connaissances acquises et le développement de compétences par l'élève le guident dans ses actions et lui servent à comprendre le monde dans lequel il évolue.

Pour y arriver, le MELS propose trois paliers constructifs :

1. Les compétences transversales (CT).

2. Les domaines généraux de formation (DGF).

3. Les domaines d'apprentissage (DA).

Les compétences transversales ont un caractère générique. Elles se déploient à travers les divers domaines d'apprentissage et doivent être promues par tout le personnel de l'école. Les domaines généraux de formation, quant à eux, rapprochent les savoirs disciplinaires des préoccupations quotidiennes de l'élève. Enfin, les domaines d'apprentissage comprennent 14 disciplines regroupées dans 5 domaines : des langues; de la mathématique, de la science et la technologie; de l'univers social; des arts et; du développement personnel (PFEQ, 2001). Le schéma 1.2.1, représente schématiquement le Programme de formation de l'école québécoise et résume l'idéologie du MELS relativement à enseignement offert aux élèves pour qu'ils développent une vision juste du monde qui les entoure. L'ensemble des activités se 
déroulant à l'école doit tenir compte de ce principe de cohérence entre les trois éléments constructifs du programme.

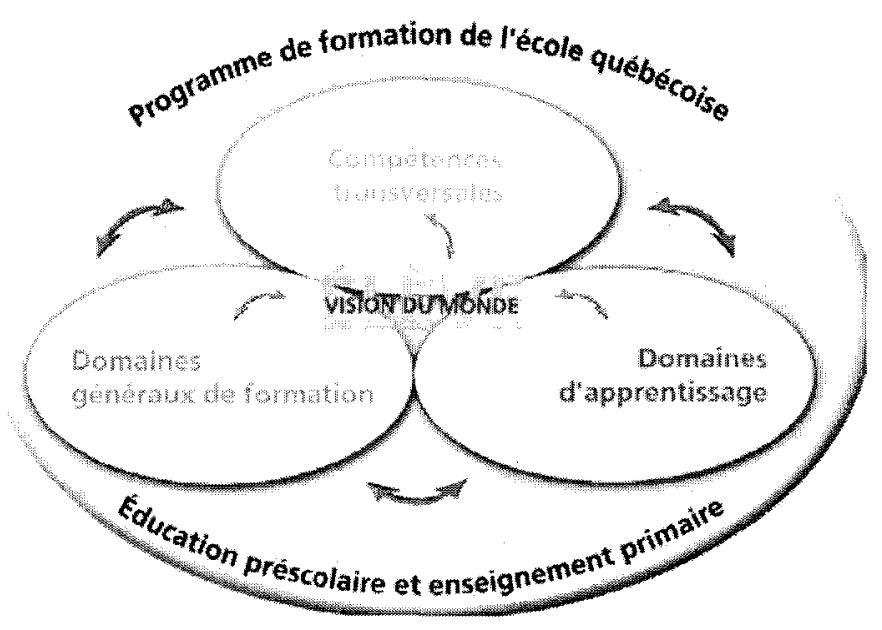

Schéma 1.2.1 : Représentation schématique du Programme de formation de l'école québécoise (PFEQ, 2001)

Ainsi, la place de la santé se situe au niveau des trois paliers. Premièrement, deux compétences transversales d'ordre personnel et social (7 et 8 ) touchent particulièrement le développement de la personnalité du jeune. Deuxièmement, un domaine général de formation, soit Santé et bien-être, s'intéresse directement à cette dimension de la vie du jeune et troisièmement, afin de préciser les savoirs essentiels dans le domaine, le programme de formation établit deux domaines d'apprentissage qui abordent les thématiques liées à la santé : le domaine de la mathématique, de la science et de la technologie et le domaine du développement personnel qui comprend l'éducation à la santé. «Plus que jamais, l'école joue un rôle important 
dans la compréhension des enjeux liés à la santé et au bien-être ainsi qu'à l'adoption de saines habitudes de vie »(PFEQ, 2001, p. 44).

Le 18 mai 1999, un communiqué de presse envoyé par le cabinet du ministre d'État à l'Éducation et à la Jeunesse mentionne qu'au primaire, le temps consacré à l'enseignement des arts et de l'éducation physique peut aller jusqu'à 5,5 heures par semaine, au premier cycle, et jusqu'à 9,5 heures aux deuxième et troisième cycles. Cela s'inscrit dans le même ordre d'idées voulant que la responsabilité liée à la santé et au bien-être des jeunes déborde largement le cadre des seules interventions propres au programme d'éducation physique et à la santé (PFEQ, 2001). Toutefois, une étude québécoise menée par Dale, Corbin et Dale (2000) soutient que malgré l'instauration du renouveau pédagogique, la diminution du temps consacré au cours d'éducation physique n'est toujours pas compensée par une augmentation de la pratique d'activités physiques en dehors des heures de cours.

Smith (1940, in Raiola et al., 1999), Neill et Richard (1998) et Horwood (1999) considèrent que les activités de plein air, tout comme l'éducation physique (MEQ, 1981), offrent de belles occasions pour explorer sous un angle différent plusieurs domaines d'apprentissage. À cet effet, plusieurs types de programmes ont déjà été expérimentés aux Etats-Unis, en Australie et dans l'ouest du Canada (Miles, 1999). Ces programmes empruntent différentes philosophies celles de l'éducation en plein air (outdoor education), des programmes d'aventure (adventure education) et de l'apprentissage par l'expérience (experiential learning). 
Dans l'optique de favoriser une éducation à la santé viable en milieu scolaire, il semble primordial de définir et de comprendre chacune des philosophies afin d'établir un mode d'intervention adapté aux exigences du PFEQ.

\subsection{Développer un mode d'intervention viable visant les axes de développement du DGF Santé et bien-être et le développement des compétences personnelles et sociales}

À ce jour, la littérature rapporte peu d'interventions ou de programmes en milieu scolaire ayant permis de rehausser ou maintenir la santé des jeunes (Ayotte, 1995, p. 18; Motta, 1998). Le PFEQ (2001) propose par l'intermédiaire des domaines généraux de formation d'aborder la Santé et bien-être comme une des grandes problématiques à laquelle s'exposent (ou sont exposés) les jeunes puisqu'elle touche aux dimensions de la vie contemporaine (PFEQ, 2001), tout comme l'exploration du monde médiatique, l'environnement et la consommation.

La thèse de Turcotte (2006) met en lumière les conditions favorables à la mise en œuvre d'un programme d'éducation à la santé dans le système scolaire. L'Institut national de la santé et de recherche médicale (2001) a identifié plusieurs de ces conditions qui sont souvent absentes des préoccupations du milieu scolaire. Les principales sont présentées ci-dessous :

- Le temps alloué doit être suffisant pour intervenir efficacement; 
- l'information ne suffit pas puisque l'amélioration des connaissances ne conduit pas nécessairement à des changements de comportements;

- les méthodes doivent être diversifiées et solliciter la participation active et interactive des élèves pour les impliquer dans leurs apprentissages;

- la clarification des valeurs et des attitudes ainsi que le développement de l'estime de soi constituent des facteurs éthiques, mais aussi des facteurs d'efficacité.

Il existe plusieurs façons d'intervenir en milieu scolaire selon qu'on travaille sur des attitudes ou des comportements (Brassard \& Mongeau, 2004; Bastien, 2001; Godin, 2002). Ainsi, l'option à envisager pour modifier les conduites antagonistes en matière de santé est de structurer l'action autour d'éléments psychosociaux et de déterminants de la santé (Hamel, 2001) qui reposent sur des facteurs clés du développement individuel et environnemental (Martin \& Arcand, 2005) et l'adoption de saines habitudes de vie.

Bien que plusieurs approches soient proposées pour développer des programmes en promotion de la santé, le choix d'un mode d'intervention doit d'abord s'appuyer sur l'identification du ou des facteurs de risque ou de protection qui expliquent le comportement de la population à l'étude (Godin, 2002).

Une des assises importantes pour permettre aux jeunes de prendre des décisions éclairées et de refuser de s'engager dans des comportements destructeurs 
pour eux et pour la société se fonde sur la perception positive d'eux-mêmes, de leurs habiletés et de leurs compétences (Chamberland et al., 1996).

Insel, Roth, Peterson, Rollins et Peterson (1997) évoquent que maintenir un haut degré d'estime de soi et savoir gérer son stress de façon efficace représentent deux éléments primordiaux afin de conserver une bonne santé. Plus récemment, dans l'élaboration de l'AES, Martin et Arcand (2005) ont exposé, dans un graphique, les facteurs clés du développement des jeunes (annexe 1) sous les angles individuel et environnemental. Parmi les facteurs individuels s'inscrivent l'estime de soi, les compétences sociales, les habitudes de vie et les comportements sains et sécuritaires. Ces facteurs convergent notamment vers les intentions éducatives du DGF Santé et bien-être et les composantes des compétences transversales 7 et 8 .

\subsubsection{L'estime de soi : facteur de protection en santé et en éducation}

Parmi les facteurs de protection répertoriés, Hamel (2001) et Duclos (2001) évoquent qu'il y a peu de domaines de la santé et du bien-être qui ne soient pas réputés avoir un lien avec le concept de soi ou l'estime de soi. Par exemple, l'image corporelle est un facteur de modélisation du concept de soi (CAAO, 2003); une modification à l'égard de ce paramètre, par exemple, sous forme de distorsion cognitive, influence 1'autoévaluation et par conséquent, l'estime de soi (Eide, 1982; Taggart, 1977, tiré de Ledoux et al., 2002, chapitre 14). Aussi, selon Viau (1999), l'estime de soi influence la motivation des jeunes envers l'école, de même qu'envers 
la pratique d'activité physique (Famose, 2001). Or, Breton et al. (2002) soulignent que les activités d'apprentissage deviennent plus significatives lorsque l'enfant trouve une motivation dans l'activité pratiquée.

Les auteurs sont sans équivoque quant à l'intérêt de travailler au développement de l'estime de soi au cours de l'enfance ainsi qu'au début de l'adolescence afin de favoriser l'adaptation sociale et de prévenir la détresse psychologique à l'adolescence (Breton et al., 2002). Plusieurs auteurs soulèvent que le degré d'estime de soi d'un individu influence sa santé mentale, sa santé physique et son bien-être (Ayotte, 1995; Breton et al., 2002; Neill \& Richard, 1998; Hamel, 2001).

Par conséquent, il semble exister une relation causale entre l'estime de soi et la prise en charge de sa santé et de son cheminement scolaire. Être en santé ne se résume pas uniquement à bouger, bien manger et à ne pas fumer, il faut aussi savoir composer avec ses sentiments personnels de façon positive et optimiste.

Dans la même visée que le PFEQ (2001) Breton et al. (2002) déclarent que le niveau de compétence dans les domaines jugés importants par l'enfant ou l'adolescent constitue un déterminant essentiel à la consolidation de son estime personnelle. 


\subsection{L'aventure éducative en plein air : un atout pour le MELS}

Par le renouveau pédagogique, le MELS désire influencer positivement la réussite et le bien-être des jeunes à l'école. L'enquête sociale sur la santé des jeunes effectuée en 1999 note que la réforme permet à chaque école, sous la supervision du conseil d'établissement, de concevoir la grille horaire. Barnett et Hamel (2002) soulignent qu'il est désormais possible pour chaque établissement d'enseignement de décider du temps dévolu à l'éducation physique et à la santé. De plus, la responsabilité d'amener les élèves à être actifs tous les jours et à adopter de saines habitudes de vie incombe maintenant à toute l'équipe-école (PFEQ, 2001). Ainsi, dans le but de favoriser la santé et la réussite des jeunes, le renouveau pédagogique laisse place à l'élaboration de projets adaptés au milieu et impliquant l'ensemble de l'équipe-école.

C'est dans cette optique que l'idée d'utiliser l'éducation à l'aventure en plein air sera exploitée. Horwood (1999) et Priest et Gass (1997) ont fait valoir que ce type d'intervention favorise l'action globale et renforce les capacités individuelles par le développement de compétences intrapersonnelles (développement émotionnel) et interpersonnelles (développement social) chez les jeunes.

L'éducation par l'aventure s'inscrirait donc directement dans les visées du PFEQ en proposant une plateforme propice aux apprentissages de toutes sortes. Les modèles expérimentaux démontrent que les activités éducatives en plein air recèlent 
un grand potentiel d'application concernant notamment les éléments éthique, mathématique et scientifique (Hopkins \& Putnam, 1997; Neill \& Richard, 1998).

L'intervention éducative par l'aventure en plein air, selon Neill (2000), propose une occasion de relever des défis, d'expérimenter le succès et d'accroître son estime personnelle. Plus un individu connaît son potentiel et ses limites, plus il s'aventurera dans des expériences lui demandant d'exploiter ses habiletés et ses capacités en tant qu'être humain. L'aventure éducative en plein air offre aux jeunes un exotisme qui favorise l'épanouissement, suggère l'exploration de son potentiel (Ann Klint, 1999) et contribue à enrichir les relations avec les pairs et l'autorité, et particulièrement avec les enseignants.

L'introduction dans le milieu scolaire d'activités éducatives en plein air présente plusieurs atouts puisque ce mode d'intervention recèle d'innombrables possibilités sur les plans personnel et social et permet l'actualisation des domaines d'apprentissage et ce, quel que soit le niveau scolaire. Plusieurs auteurs (Neill \& Richard, 1998; Hopkins \& Putnam, 1997) considèrent que l'aventure éducative en plein air permet au jeune d'explorer ses aptitudes physiques et mentales, l'amenant à se différencier de ses pairs et à se façonner une identité (Reasoner, 1982). Dans la théorie éducative de Dewey (Houssaye, 1994), le concept d'intérêt est fondamental aux apprentissages. Ainsi, l'école se doit de trouver des façons qui solliciteront la curiosité naturelle de l'enfant. 


\subsection{L'intérêt et l'originalité de cette recherche}

Au Québec, à ce jour, il existe très peu d'interventions efficaces en matière de santé auprès des jeunes de 10 à 12 ans (Ayotte, 1995; Bastien, 2001). Turcotte (2006) soulève aussi que l'inclusion de l'éducation à la santé en milieu scolaire est loin d'être acquise par l'ensemble de l'équipe-école. Toutefois, à plusieurs reprises dans le PFEQ, il est mentionné que la santé est aussi l'affaire des titulaires de classe. L'éducation par l'aventure en plein air présente, à notre avis, un enrichissement pour le curriculum, de même qu'une fenêtre ouverte sur l'éducation à la santé en milieu scolaire. Il semble évident que ce mode d'intervention exigera une réelle collaboration de la part des titulaires de classe et de l'ensemble de l'équipe-école (PFEQ, 2001). Toutefois, les situations d'apprentissage développées au cours de la séquence d'aventure pourraient sans doute devenir des outils utiles à l'acquisition de connaissances, et du développement des compétences et ce, tant pour l'apprenant que pour l'enseignant. Le plein air sollicite les sens, éveille la curiosité et surtout offre aux jeunes la pratique d'activité physique sous un angle plus ludique, privilégiant la découverte, le plaisir et la convivialité (Beunard, 1999; Dugual, 1999; Lapointe et al., 2000).

La littérature rapporte que les retombées des modèles expérimentaux sont multiples. À titre d'exemple, Neill et Richard (1998), Chabonneau (2001) et Kraft (1999) mentionnent que les activités d'exploration et d'aventure éducative en plein air invitent l'apprenant à découvrir ses habiletés physiques, à utiliser ses aptitudes 
mentales et à interagir avec ses pairs dans un environnement naturel. Selon Ann Klint (1999) et Neill (2000), ce type d'activités contribuerait à bonifier l'état de santé globale en augmentant la souplesse, en améliorant l'endurance cardiovasculaire et musculaire, et en favorisant l'éclosion d'un sentiment de bien-être.

Ce mode d'intervention peut s'adresser à une clientèle préadolescente, perçue par plusieurs auteurs comme une clientèle à risque en matière de santé (Ouellet \& Stan, 2002; Morissette et al., 2002; Barnett \& Hamel, 2002; Breton et al., 2002).

\subsection{Question de recherche et objectifs}

L'introduction d'ateliers éducatifs par l'aventure en plein air dans le programme de formation de l'école québécoise contribue-t-elle à développer une conscientisation en matière de santé chez un groupe d'élèves du troisième cycle du primaire?

La question de recherche étant exposée, nous avons formulé les trois objectifs suivants :

1. Mettre en place une séquence d'éducation par l'aventure en plein air en accord avec les assises du PFEQ dans le but de développer une conscientisation en matière de santé chez un groupe d'élèves du troisième cycle du primaire.

2. Identifier les retombées, potentiellement positives, d'un tel mode d'intervention sur la santé globale des jeunes en utilisant les assises du DGF Santé et bien-être 
et deux compétences transversales d'ordre personnel et social, soit structurer son identité et coopérer.

3. Explorer les retombées de la séquence d'aventure et d'apprentissage sur les facteurs de développement individuel du jeune. 


\section{CHAPITRE 2}

\section{Cadre conceptuel}

Lorsqu'il est question de promotion ou d'éducation à la santé, les théoriciens considèrent l'individu dans son intégralité. En 1986, deux ans après que l'Organisation mondiale de la santé (OMS) ait statué sur une définition de la promotion de la santé, la Charte d'Ottawa donne naissance au concept de promotion de la santé qui se définit comme "un processus qui confère aux populations les moyens d'assurer un plus grand contrôle sur leur propre santé, et d'améliorer celleci ». ((Promotion de la santé), 1986, p. 233). Pour y arriver, plusieurs stratégies sont imaginées : publicités, programmes d'éducation à la santé, actions politiques et communautaires, etc.

Le présent chapitre, dans un premier temps, propose des définitions concernant la promotion et l'éducation à la santé, puis expose les éléments clés du PFEQ (2001) ayant le potentiel de renforcer positivement les facteurs clés de développement en matière de santé. Dans un deuxième temps, dans le but de développer une stratégie d'intervention en accord avec les assises du PFEQ, nous porterons un regard sur des 
pratiques d'intervention éducatives potentielles, tel l'apprentissage expérientiel en plein air, qui seront mis en parallèle avec certaines théories éducatives.

\subsection{Définition de la santé}

Dans le but de s'engager dans un processus d'éducation à la santé, il est essentiel d'explorer la signification de la santé dans son ensemble. Le Petit Robert (Rey, Rey-Debove \& Cottez, 1990) définit la santé comme «un bon état physiologique, un fonctionnement régulier et harmonieux de l'organisme pendant une période appréciable ». Le MEQ (2001), dans le PFEQ, mentionne qu'être en bonne santé, c'est réunir les conditions physiques et psychologiques nécessaires à la satisfaction de ses besoins et à la réalisation de projet. En promotion et en éducation à la santé, les théoriciens considèrent l'individu dans sa globalité à travers les six dimensions suivantes: physique, mentale, émotionnelle, sociale, spirituelle et sexuelle (Naidoo \& Wills, 1994). Précisément, l'aspect physique concerne le corps, l'aspect mental concerne la capacité de jugement, le côté émotionnel s'intéresse à la reconnaissance (perception) des émotions, l'aspect social porte sur la place de l'individu dans ses relations avec autrui, la santé spirituelle reconnaît la capacité de mettre en pratique une croyance quelconque (divinité, superstition, adage..) et la santé sexuelle s'intéresse à l'expression sexuelle de l'individu concerné. La société et l'environnement agissent comme des infrastructures essentielles au développement et au maintien de la santé des individus et, par conséquent, des 
populations. Elles sont représentées, au schéma 2.1.1, par deux cercles concentriques situés autour du noyau formé des six dimensions.

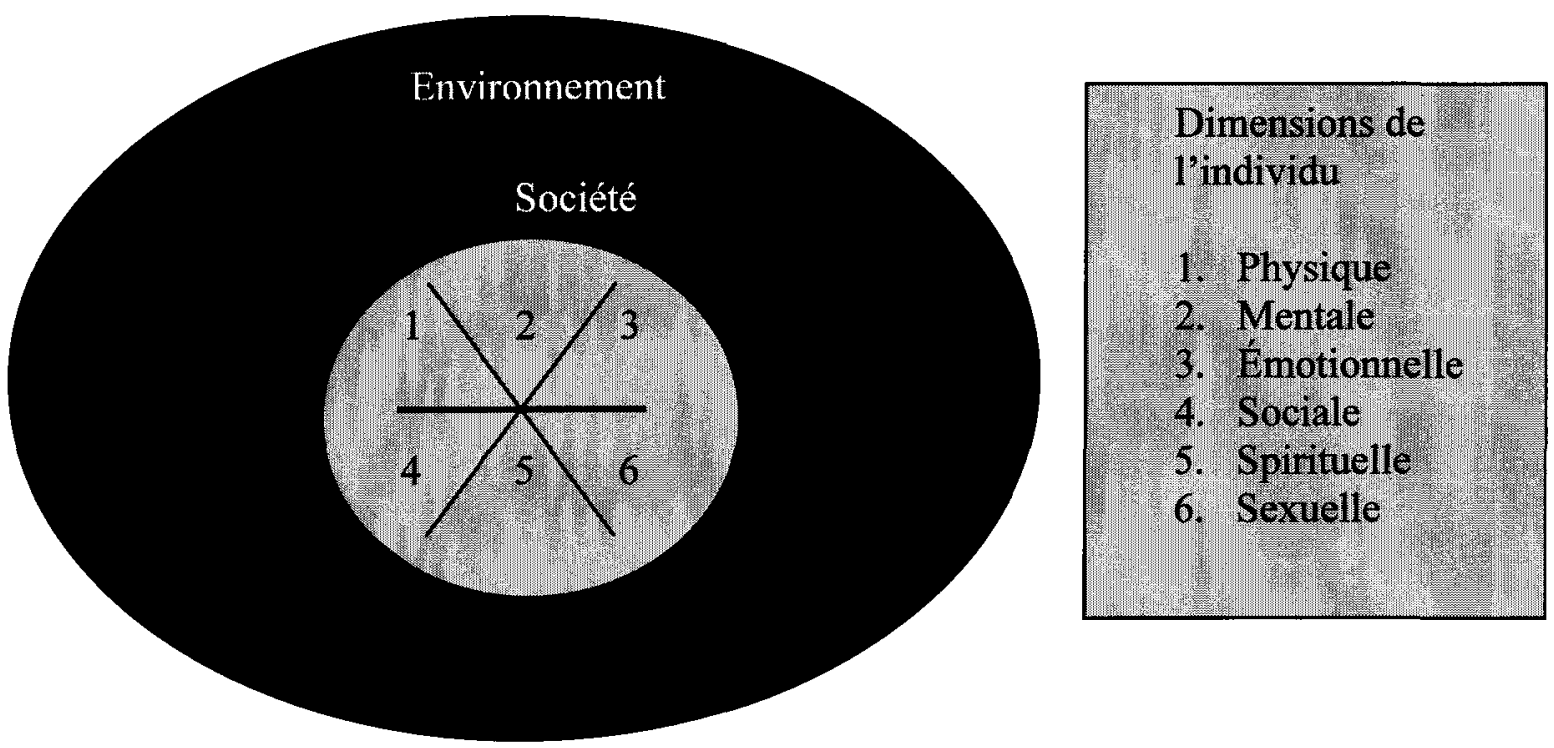

Schéma 2.1.1 : Dimensions de la santé (Naidoo \& Wills, 1994)

Dans une perspective globale de la santé, les stratégies déployées doivent être soutenues par la société et viser un environnement sain. Ce concept multifactoriel conduit la démarche de la présente recherche vers l'utilisation d'une approche holistique, utilisant un cadre naturaliste.

La santé est un large concept qui inclut la notion des habitudes de vie. Selon Deschênes (2001), la notion d'habitudes de vie est vague, mais semble comprise de tous. Bourdieu (1992), sous une vision plus épistémologique de l'action sociale définit 1'expression « habitude de vie » directement à partir de la racine latine du mot « habitus », soit un système qui est socialement constitué de structures et de 
dispositions structurantes qui sont apprises à partir des pratiques (Brassard \& Mongeau, 2004). De Koninck (2004) mentionne que le développement d'une habitude exige un apprentissage (raison) en harmonie avec la nature, car «sans apprentissage la nature est aveugle, dit-il, cependant que l'apprentissage sans nature est imparfait et la pratique sans les deux ne donne rien (p. 22)». Il poursuit en mentionnant qu'à la suite de la compréhension de l'exercice par la pratique, l'individu en vient à poursuivre et à développer l'habitude. Ainsi, plus l'individu commence tôt son apprentissage de la pratique, plus les gestes exécutés deviendront des habitudes. Comme les habitudes s'acquièrent principalement au cours de l'enfance et de l'adolescence, la préadolescence est donc une période charnière, puisque les pairs prennent de l'importance, le jeune se trouve en quête d'autonomie et redéfinit les relations qu'il entretient avec ses parents (Claes, 1994; Hamel, 2001).

\subsubsection{La préadolescence, une cible importante en matière de promotion de la santé}

Les jeunes de 10 et 12 ans se trouvent devant de nombreux choix au regard de leur avenir, notamment en ce qui a trait aux habitudes de vie et à la consommation de substances psychoactives (Claes, 1994; Morissette et al., 2002).

Sur le plan physique, les changements corporels mettent à rude épreuve le concept de soi physique qui inclut notamment l'estime de soi, l'image corporelle et par conséquent, la pratique d'activité physique (Famose, 2001). La conscience de soi

devient une dimension centrale à la croissance psychique des jeunes (Brassard \& 
Mongeau, 2004). Comme le mentionne Hamel (2001), c'est à la fin de l'adolescence que le soi devient un système organisé. Les choix personnels et moraux émergent alors des croyances et des valeurs intégrées. Par conséquent, la clientèle préadolescente semble être celle à privilégier pour agir en amont sur le développement de plusieurs comportements néfastes en matière de santé.

\subsubsection{Une vision de la santé et du bien-être selon Maslow}

En 1972, Maslow perçoit la santé comme une déficience demandant à être comblée afin d'atteindre un état de bien-être (Deschênes, 2001). Sa théorie propose une hiérarchie des besoins, qu'il représente sous forme d'une pyramide. Maslow (1972) considère que le bien-être de la personne passe indéniablement par la satisfaction de ses besoins classifiés en cinq paliers. Au premier plan de la pyramide (figure 2.1.1), Maslow place la survie qu'il explique comme étant les besoins physiologiques tels que manger, dormir, respirer et bouger, etc. Au second plan, réside le besoin de sécurité, tel se loger. Maslow (2004) affirme que le besoin de sécurité trouve aussi souvent une expression spécifique dans la quête d'un protecteur. Au troisième palier, il situe le besoin de socialiser qui inclut le besoin d'amour et le sentiment d'appartenance (Reasoner, 1982). Le quatrième niveau constitue le besoin d'estime dans sa globalité puis, finalement, au sommet de la pyramide, trône le besoin d'actualisation, d'autoréalisation ou d'accomplissement. Ce besoin est comblé lorsque l'individu « fait ce pour quoi il est compétent, doué ». 
Par exemple, pour s'actualiser (s'accomplir) un musicien doit jouer de la musique (Maslow, 2004).

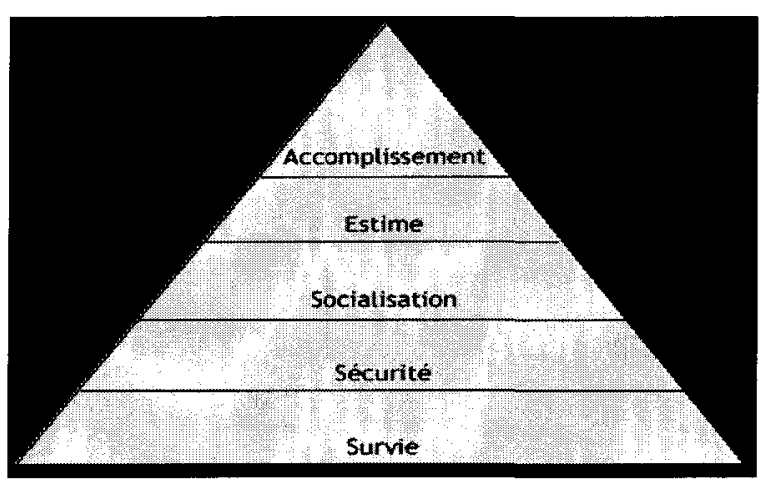

Figure 2.1.1 : Pyramide de Maslow

Ainsi, pour que l'individu se réalise pleinement, Maslow croit que l'ensemble des besoins présentés à la figure 2.1.1 doit être satisfait de façon convenable en suivant la hiérarchie proposée.

\subsubsection{Un parallèle de la théorie de Maslow avec le DGF Santé et bien-être du PFEQ}

En pratique, la théorie de la pyramide de Maslow se retrouve dans le PFEQ au travers du domaine général de formation (DGF) Santé et bien-être. Par exemple, le premier axe du DGF Santé et bien-être aborde la conscience de soi des besoins fondamentaux. C'est-à-dire les besoins physiques (physiologiques), le besoin de sécurité, le besoin d'acceptation (socialisation), le besoin d'épanouissement (estime) et finalement le besoin d'actualisation qualifié par Maslow comme le besoin 
d'accomplissement. Deschênes (2001) note dans son mémoire qu'un étudiant qui a faim, soif ou froid ou encore qui se sent triste sera incapable d'offrir un plein rendement scolaire, ce qui empêchera sa réalisation personnelle. Il va de soi qu'avant de combler ses besoins quels qu'ils soient, l'individu doit en premier lieu apprendre à les percevoir, à les identifier, à en prendre conscience (De Koninck, 2004).

En 1998, le groupe interministériel sur les curriculums, volet Santé et bien-être dirigé par Arcand et al. (1998, p. 2), définit la santé comme « une mesure dans laquelle une personne ou un groupe peut d'une part, réaliser ses ambitions et satisfaire ses besoins et d'autre part, évoluer dans son milieu ou s'adapter à celuici. "). Dans le même ordre d'idées, Bilodeau (2000) explique que la nature et l'aventure encouragent les participants à développer des habiletés de survie, à réaliser des actions favorisant leur sécurité, par exemple, la construction d'un campement, à accomplir des tâches, seuls ou en groupe, qui exigent un dépassement personnel, par exemple l'ascension d'une montagne. Toutes ces expériences contribueraient à l'essor de multiples compétences associées à combler l'ensemble des besoins inscrits dans la pyramide de Maslow.

Cette perspective chemine ainsi vers une vision holistique de la santé qui met en valeur l'ensemble des ressources sociales et personnelles des individus, puis rejoint sur plusieurs plans les assises du PFEQ. 


\subsubsection{Une définition de la promotion de la santé en lien avec l'éducation à la santé}

En 1974, l'expression "promotion de la santé » est utilisée pour la première fois par Marc Lalonde, alors ministre canadien de la Santé et du Bien-être social. Par la suite, en 1984, l'Organisation mondiale de la santé (OMS) statue sur une définition. La promotion de la santé est un concept unifié qui reconnaît le besoin de changement des conditions de vie. La promotion de la santé représente une stratégie médiane entre les gens et leur environnement qui est teintée de choix personnels et de responsabilités sociales, afin de créer un futur en santé.

En 1986, cette définition est formalisée lors de la première conférence internationale pour la promotion de la santé (Institut national de la santé et de la recherche médicale, 2001).

Naidoo et Wills (1994) considèrent l'éducation à la santé comme une partie du concept de promotion. La promotion de la santé couvre l'ensemble des aspects ou des activités visant l'amélioration de l'état de santé de l'individu et de la communauté. Brassard et Mongeau (2001) soutiennent que l'éducation à la santé se distingue principalement par sa volonté à rendre l'individu autonome dans l'ensemble de ses actions, notamment grâce à une forte estime personnelle (French, 1990 dans Naidoo \& Wills, 1994). 
L'état actuel des connaissances sur la santé et le bien-être des jeunes est très avancé. À titre d'exemple, Arcand et ses collaborateurs (1998) expliquent que certains facteurs ont un effet positif sur la santé et le développement du jeune. Ces facteurs sont appelés facteurs de robustesse, facteurs de protection (Ledoux, 2002) et déterminants positifs (Jutras \& Bisson, 1994). Arcand et al. (1998) estiment que ces facteurs jouent un rôle positif tant dans le maintien de la santé qu'en matière de réussite scolaire. Avoir de saines habitudes de vie, adopter des comportements sécuritaires, bénéficier d'une estime de soi positive, avoir confiance en soi, obtenir un soutien social de qualité et avoir des rapports sociaux équitables entre les garçons et les filles sont des exemples de facteurs et de déterminants réputés pour avoir un impact positif tant sur le bien-être que sur la réussite scolaire (Arcand et al., 1998; Ledoux, 2002; Jutras \& Bisson, 1994; Hamel, 2001; Martin \& Arcand, 2005). Turcotte (2006) rappelle que la tendance principale de l'éducation à la santé demeure centrée sur les comportements. Toutefois, selon Naidoo et Wills (1994) et Chamberland et al. (1996), il est primordial de respecter les normes culturelles ainsi que les contraintes économiques et sociales dans lesquelles évoluent les jeunes puisque ces facteurs influencent indéniablement l'orientation des choix en matière de santé.

\subsubsection{Les modèles et théories en matière d'éducation à la santé}

Selon l'organisme français l'Inserm (2001), l'éducation à la santé est influencée par plus de 20 théories et modèles principalement axés sur la 
compréhension des comportements en matière de santé. L'Inserm (2001) regroupe les modèles en huit catégories distinctes, passant du modèle biomédical aux théories de l'apprentissage social, des représentations sociales et des modèles intégrateurs (Turcotte, 2006). Godin (2002) estime, à la suite d'une analyse approfondie de ces modèles et théories, que seulement cinq modèles représentent des références valables pour permettre un choix éclairé quant à la méthode d'intervention.

C'est au cours des années 1950 que le modèle des croyances relatives à la santé (Health Belief Model, HBM), développé aux États-Unis, fait son apparition (Rosenstock 1974, dans Turcotte, 2006). En 1975, Fishbein et Ajzen mettent en place la théorie de l'action raisonnée et, deux ans plus tard, au même moment que Bandura qui propose les théories sociales cognitives, Triande suggère la théorie des comportements interpersonnels (Bastien, 2001; Turcotte, 2006). Tous ces modèles dominants en éducation à la santé reposent largement sur le développement de compétences personnelles, l'intention et les habitudes développées.

Les modèles et théories en matière d'éducation à la santé (Bastien, 2001, Turcotte 2006) sont présentés ci-dessous :

- Le modèle de croyances relatives à la santé (Health Belief Model, HBM) pose comme prémisse qu'un individu possédant des connaissances minimales en matière de santé, et considérant celles-ci comme une dimension importante de sa vie, entreprendra les actions jugées pertinentes pour la conserver; 
- la théorie de l'action raisonnée va dans le même sens en insistant sur l'intention de la personne à poser ou non une action donnée. Ici, la composante attitude porte sur les conséquences positives ou non à adopter un comportement donné, et la composante normative insiste sur la motivation à se conformer à la pression de l'entourage;

- le modèle des théories sociales cognitives met en lumière la croyance en l'efficacité personnelle à adopter un comportement social ou relié à la santé. La croyance en l'efficacité personnelle est un bon indice du taux de réussite et de persévérance.

- La théorie des comportements interpersonnels soulève que plusieurs comportements devenus par la pratique plus ou moins automatiques constituent un facteur appréciable de prédiction. L'habitude est une composante extrêmement importante, car la plupart des comportements sociaux réalisés quotidiennement sont sous le contrôle des habitudes.

Toutes ces théories vont dans le même sens et perçoivent les comportements reliés à la santé sous un angle social et comportemental, plutôt que sous l'angle exclusif de leur lien avec la santé et la maladie.

Ainsi dans le but d'avoir une vision plus holistique des théories présentées précédemment, nous utiliserons le modèle de Godin (2002) qui intègre ces principales théories. Ce modèle, présenté à la figure 2.1.2, repose sur l'importance de l'intention, c'est-à-dire la motivation à adopter un comportement. Toutes les variables du modèle sont, quant à elles, des facteurs qui influencent l'intention. Ces 
facteurs sont classés en trois catégories : les attitudes, les normes perçues et le contrôle perçu sur le comportement. Les variables externes, quant à elles, exercent une influence sur le comportement par l'entremise de l'une ou l'autre des trois variables précédentes.

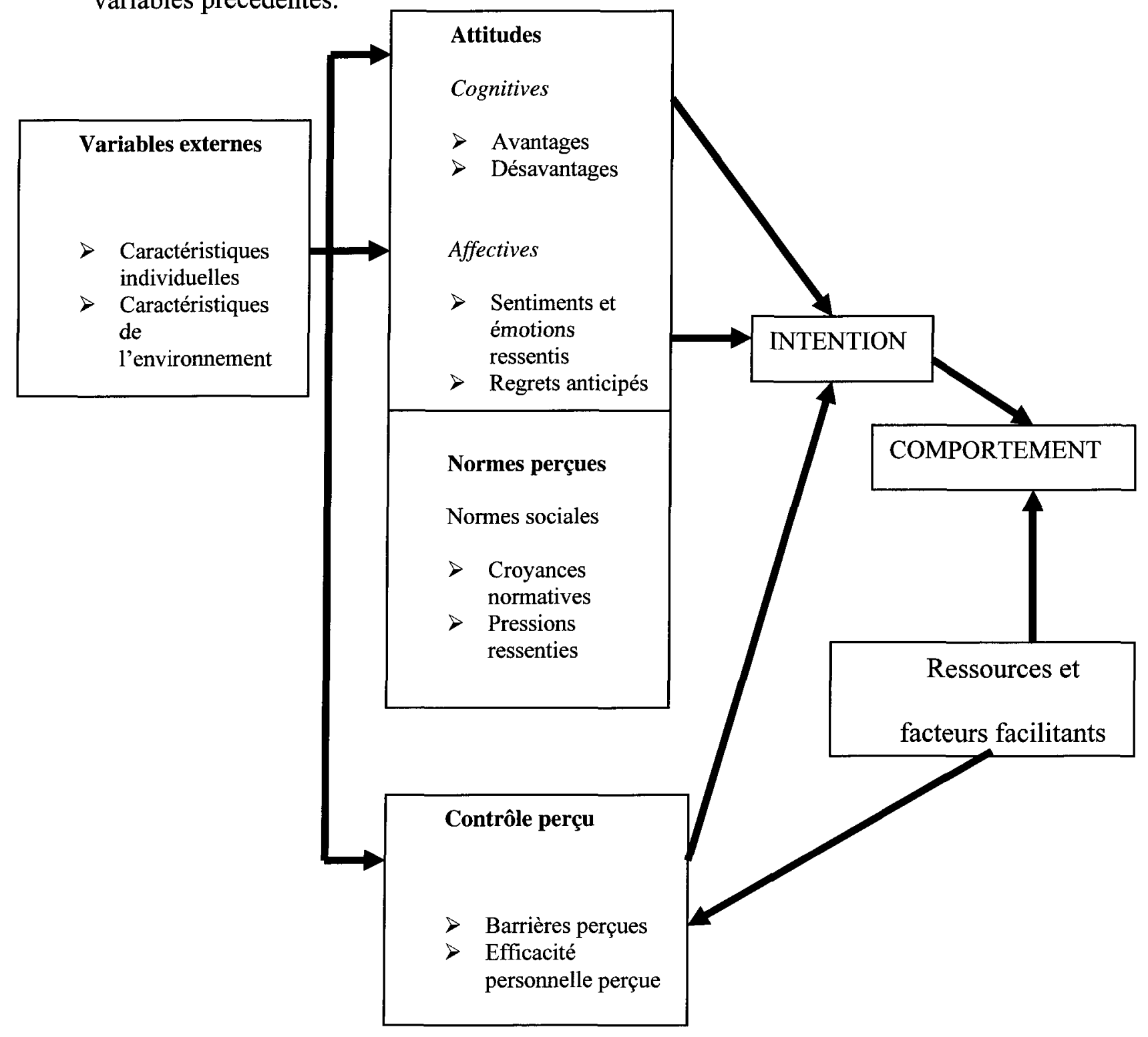

Figure 2.1.2: Modèle intégrateur de Godin (2002) en lien avec les différents modèles et théories de l'éducation à la santé privilégiés en Amérique du Nord (Turcotte, 2006, p. 37) 
Développer l'habitude d'accomplir des actions gagnantes en matière de santé, travailler sur l'efficacité personnelle et les compétences individuelles sont des facteurs essentiels à considérer dans l'élaboration d'action (stratégie d'intervention) en matière de promotion de la santé.

Turcotte (2006) juge que le comportement serait prédit par l'intention, pourvu que les conditions soient favorables à la concrétisation de cette dernière. La responsabilité individuelle par rapport aux événements qui agissent sur la santé demeure un paramètre important. À ce propos, Parcel, Kelder, et Basen-Engquist (2000) présentent l'empowerment, traduit dans ce document par l'autonomisation, comme un modèle en émergence. Ce modèle propose un processus de changement axé sur l'initiative des élèves et non sur celle des enseignants au même titre que le PFEQ qui met l'élève au cœur de ses apprentissages. Pour favoriser les habiletés d'autonomisation chez les jeunes, il est primordial de développer, selon Tones et Green (2004), les habiletés cognitives, c'est-à-dire d'accroître, à la suite d'une analyse de la situation, les connaissances et les capacités à prendre des décisions favorisant la santé, le bien-être et la réussite. Pour représenter l'autonomisation, nous avons choisi d'utiliser le modèle de santé par l'action (Health Action Model) présenté à la figure 2.1.3, car il rejoint les idéologies des concepteurs du PFEQ. En effet, ce modèle intègre les principes de démarche réflexive dans le développement de comportements sains. 
Tones et Green (2004) expliquent que la personnalité de l'individu résulte d'un discernement spontané propre à chacun à l'égard des différents systèmes (croyances, normes et motivation) qui sont tous sous l'influence de son environnement physique, socio-économique et culturel. Ensemble, ces quatre systèmes (croyances, normes, motivation et le moi) déterminent la probabilité que l'individu développe une action saine ou non en matière de santé. Ceci dépendra du nombre de facteurs facilitants, et cela inclut les connaissances et les habiletés nécessaires au choix d'actions.

L'individu adopte donc des comportements sains, à la suite de l'acquisition de connaissances et à la suite du développement de diverses habiletés psychomotrices et sociales. La conscience de soi et des conséquences de ses choix personnels sur sa santé et son bien-être se confirment par la perception positive des actions qui encouragent la réussite et l'épanouissement de l'individu.

La figure 2.1.3 présente le modèle de la santé par l'action (HAM). C'est donc à partir de ce dernier modèle que nous allons construire la stratégie en gardant en tête l'importance de favoriser l'acquisition de connaissances et le développement de compétences personnelles, dont les habiletés psychomotrices et sociales. Des écrits en matière d'éducation à la santé expliquent que la connaissance de phénomènes et la compréhension de l'action posée favorisent la capacité d'affirmation de l'individu et développent son esprit critique, notamment à l'égard de son environnement (physique, socio-économique et socioculturel) (Tones \& Green, 2004; Inserm, 2001). 
Le modèle de santé par l'action identifie les clés psychologique, sociale et environnementale qui influencent l'individu dans l'adoption de comportements favorisant la santé et le bien-être ou de comportements déviants en matière de santé. Il comprend deux sections majeures, c'est-à-dire les systèmes qui contribuent à modifier les intentions behaviorales et les facteurs qui déterminent la probabilité que celles-ci soient modifiées dans la pratique. L'autonomisation devient alors centrale à la première de ces deux sections, car l'interaction des quatre systèmes, c'est-à-dire les croyances, la motivation, l'influence des normes et la personnalité, détermine la probabilité qu'un individu développe une intention et adopte une action santé. Ce phénomène se confirme lorsque certains facteurs facilitants tels que les connaissances et les habiletés sont présents et que l'environnement est aidant. Le modèle de santé par l'action reconnaît la différence entre une action ponctuelle et routinière (Tones \& Green, 2004). 


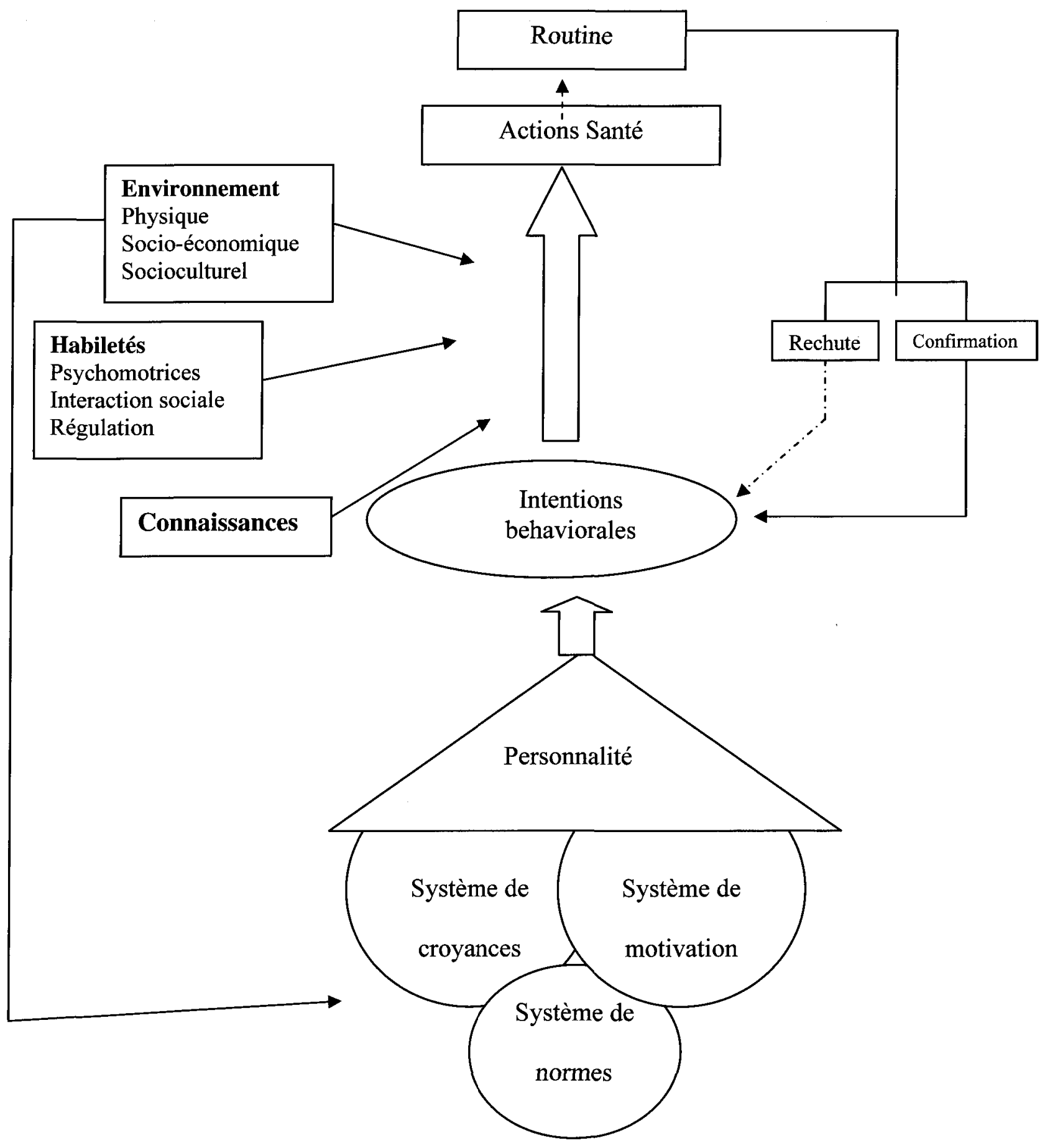

Figure 2.1.3 : Health Action Model- HAM. (Tones \& Green 2004) 
Afin d'exercer un contrôle sur eux-mêmes, et en relation avec leur bien-être, les jeunes doivent croire en leurs capacités individuelles et en leurs compétences (French, 1990 dans Naidoo \& Wills, 1994; Famose, 2001). Pour ce, il est nécessaire que les jeunes vivent des expériences leur permettant d'accroître leurs connaissances en matière de santé afin d'arriver à développer à long terme des comportements et actions dites santé.

\subsection{La santé à travers le renouveau pédagogique}

Intentionnellement ou non, c'est dans la foulée des pratiques éducatives en promotion de la santé que les concepteurs du PFEQ ont établi des assises dans le DGF Santé et bien-être, en priorisant notamment la démarche réflexive.

Pour la première fois dans l'histoire de l'éducation au Québec la santé et le bien-être se trouvent en toile de fond du programme de formation. Le Domaine général de formation Santé et bien-être sert de point d'ancrage et invite l'ensemble de l'équipe-école à offrir des activités d'éducation à la santé. Le but consiste à amener l'élève à adopter une démarche réflexive dans le développement de saines habitudes de vie sur les plans de la santé, du bien-être, de la sexualité et de la sécurité. Aussi, les activités d'apprentissage doivent permettre le développement de compétences sociales auxquelles des compétences disciplinaires se greffent afin d'optimiser la démarche (PFEQ, 2001). 
À titre d'information, le DGF Santé et bien-être se compose de trois axes de développement : la conscience de soi et de ses besoins fondamentaux, la conscience des conséquences sur sa santé et son bien-être dans ses choix personnels, et l'adoption d'un mode de vie actif et d'une conduite sécuritaire (PFEQ, 2001). Quel que soit le moyen utilisé, les assises du PFEQ cherchent à conscientiser le jeune par rapport à ses actions sur sa santé, son bien-être et sa sécurité. Compte tenu de l'importance accordée au mot «conscience» dans les écrits du programme de formation, nous avons cru bon d'en fournir une définition.

Selon le petit Robert, le mot «conscience » signifie faculté qu'a l'homme de connaître sa propre réalité et de la juger (Rey et $a l ., 1990$ ), c'est donc une perception plus ou moins claire que chacun peut avoir de son existence. Pour leur part, Shavelson, Hubner et Stanton (1976) associent le concept de soi à la perception qu'une personne a d'elle-même et constitue un élément central de la personnalité d'un individu qui détermine son comportement (Herry \& Worth Gavin, 1996).

$\mathrm{Au}$ même titre que les autres domaines généraux de formation, celui de la Santé et bien-être sert de point d'ancrage au développement des compétences disciplinaires. Par leur exploration, notamment à travers la réalisation de projet, les domaines généraux de formation rapprochent les savoirs disciplinaires des préoccupations quotidiennes de l'élève et donnent aux jeunes une plus grande emprise sur leur réalité (PFEQ, 2001; Lasnier, 2000), leur santé, leur environnement et par conséquent, leur vie. 


\subsubsection{Le domaine du développement personnel du jeune}

Le programme comporte un domaine d'apprentissage se rattachant précisément au développement personnel du jeune. Celui-ci comprend l'éducation physique et l'enseignement moral et religieux, protestant ou catholique. Les grandes lignes du domaine d'apprentissage de l'éducation physique et à la santé expliquent que cette discipline est à la fois différente et complémentaire des autres disciplines. D'une part, tout comme chacune des disciplines du programme de formation, elle amène l'élève à cerner et à intégrer dans l'action des valeurs telles que l'engagement, la solidarité, l'égalité, la dignité et le respect de soi, des autres et de l'environnement (PFEQ, 2001). D'autre part, le programme d'éducation physique et à la santé aux niveaux préscolaire, primaire et secondaire vise trois compétences. Les deux premières, Agir et interagir dans divers contextes de pratique d'activités physiques, proviennent des contenus disciplinaires de l'ancien curriculum en éducation physique (Turcotte, 2006). La troisième compétence, Adopter un mode de vie sain et actif, s'ajoute au curriculum de l'enseignant d'éducation physique. L'acquisition des savoirs essentiels ciblés par cette compétence vise l'amélioration des habitudes de vie au regard de sa santé et de son bien-être, et toute l'équipe-école doit y participer. Un partenariat doit aussi s'établir entre l'ensemble de l'équipe-école, la famille et la communauté.

Ainsi, le programme de formation se doit de vivre par et à travers l'ensemble des domaines d'apprentissage. Afin d'assurer la continuité des interventions 
éducatives en santé, le PFEQ (2001) suggère notamment l'utilisation des compétences dites transversales d'ordre personnel et social. Structurer l'identité du jeune (compétence 7) et coopérer (compétence 8) constituent les deux compétences transversales exposant l'évolution du développement d'une santé globale. En ce sens, plus l'individu se connaît, reconnaît ses besoins et les partage, selon le cas, avec son entourage, plus ceux-ci seront comblés (section 2.1.2) et par conséquent, la santé et le bien-être de l'individu en question s'en porteront mieux.

Le PFEQ (2001) et le groupe interministériel sur les curriculums, volet Santé et bien-être (Arcand et al., 1998) ainsi que d'autres études (Jutras \& Bisson, 1994; Naidoo \& Wills 1994), indiquent que le développement des compétences chez l'enfant contribue à rehausser son estime personnelle. Nous observerons donc l'évolution de ce facteur de protection, notamment à travers les manifestations des compétences transversales d'ordre personnel et social (7 et 8).

\subsubsection{L'éducation à la santé à partir du domaine général de formation Santé et bien- être}

L'intégration de l'éducation à la santé en milieu scolaire se présente sous plusieurs formes : soit une discipline unique, soit un domaine général de formation, soit un thème transversal en inclusion dans d'autres disciplines (Motta, 1998). Les récentes recherches dans le domaine (Turcotte, 2006; Motta, 1998) estiment que l'inclusion de l'éducation à la santé devrait transcender le programme de formation 
et outrepasser le contenu disciplinaire du domaine de l'éducation physique et à la santé. Toute 1'équipe-école doit devenir porteuse du message et mettre en place des projets, des tâches intégratrices et des activités d'apprentissage en lien étroit avec le domaine général de formation Santé et bien-être tout en développant les compétences transversales d'ordre personnel et social.

Lorsque les apprentissages s'explorent directement dans la vie réelle (domaines généraux de formation), ceci a un effet positif sur la motivation de l'élève (Lasnier, 2000).

2.2.2.1 Rehausser l'estime de soi en renforçant les compétences transversales d'ordre personnel et social

Pour que les compétences transversales prennent sens dans la formation du jeune, elles doivent être juxtaposées aux compétences disciplinaires dans des tâches intégratrices (Lasnier, 2000). Les domaines d'expérience de vie servent de contexte de réalisation de tâche intégratrice ou de projet.

Appuyées par des connaissances (connaissances déclaratives), les compétences seront utilisées efficacement, dans des situations ayant un caractère commun. Lasnier (2000) poursuit en mentionnant que l'expression "caractère commun » signifie qu'une compétence est contextualisée, c'est-à-dire qu'elle s'applique à une certaine 
famille de tâches. Au-delà de cette famille de tâches, l'élève doit exécuter un « transfert » de ces compétences (annexe 3).

Ainsi, la compétence est dite transversale lorsqu'elle permet d'agir efficacement dans une large variété de situations ayant des composantes disciplinaires et thématiques diverses. «Elles font partie des apprentissages essentiels. Leur développement est progressif. Elles doivent être l'objet des préoccupations de tout le personnel de l'école » (PFEQ, 2001). Cette dimension, très novatrice, du programme de formation répond à une attente sociale : amener l'élève à « apprendre à apprendre » (Sandrin-Berthon, 1997).

D'autre part, le développement de compétences servira à mieux outiller l'élève pour l'aider à faire face à la vie quotidienne et l'aidera à mieux vivre sa vie sur les plans personnel et social. Par conséquent, les compétences d'ordre personnel et social sont liées au développement et à l'affirmation de l'identité personnelle et collective de l'élève (PFEQ, 2001). Structurer son identité et coopérer touchent les dimensions sociale, mentale, physique, émotionnelle, sexuelle et spirituelle de l'être. Ces deux compétences font appel à certaines attitudes d'ouverture et de souplesse d'esprit, d'engagement et d'entraide (PFEQ, 2001). Tous les domaines d'apprentissage contribuent à développer l'identité personnelle, sociale et culturelle de l'élève. 
Le développement des compétences transversales quelles qu'elles soient se fera toujours en contextualisant les apprentissages dans une situation réelle. La plupart du temps, le domaine général de formation (DGF) est la source qui sert à contextualiser les tâches intégratrices afin de rendre les compétences disciplinaires et transversales authentiques et signifiantes. Autrement dit, l'élève s'approprie les domaines d'expérience de vie au moyen des compétences disciplinaires et transversales. Toutefois, si nous décidons d'insérer une compétence transversale dans une tâche intégratrice, il est primordial de se préoccuper de l'apprentissage explicite de celle-ci (Lasnier, 2000). Dans la présente étude, nous tiendrons compte des manifestations perçues pour les deux compétences transversales d'ordre personnel et social en portant une attention particulière à l'estime de soi.

\subsection{Les visées de l'estime de soi : définition}

L'estime de soi est perçue par les théoriciens comme l'un des principaux facteurs du développement humain (Arcand et al., 1998) ou comme la valeur qu'un individu s'accorde globalement (Legendre, 2005). Elle fait appel à la confiance fondamentale qu'a l'être humain en lui-même ainsi qu'en son efficacité. Duclos (2000) croit, pour sa part, que l'estime de soi réside dans un processus de conscientisation que le jeune développe à l'égard de sa valeur personnelle et ce, dans différents domaines. Cela suppose une conscience de ses difficultés, de ses capacités et de ses limites personnelles. En d'autres mots, pour être capable de structurer son 
identité et de coopérer avec ses pairs (compétences transversales 7 et 8 du PFEQ), l'enfant doit développer une perception réaliste de son être (Duclos, 2000).

L'estime de soi constitue un facteur de protection (Ledoux, 2002; Hamel, 2001; Arcand et al., 1998) dont la littérature fait largement état. Par exemple, le groupe interministériel sur les curriculums, volet Santé et bien-être (Arcand et al., 1998) place ce déterminant au cour de nombreuses stratégies visant à rendre les individus en mesure d'assumer leurs responsabilités, de s'affirmer, de savoir répondre à leurs besoins, d'avoir des buts et de prendre les moyens pour les atteindre. Ce groupe considère qu'une forte estime de soi constitue une arme pour prévenir de nombreux problèmes tant en santé qu'en éducation. Dans le même ordre d'idées, Hamel (2001), tout comme Duclos (2000), considère que l'estime de soi constitue un déterminant majeur tant en santé qu'en éducation, mais soulève que les théoriciens se sont en premier lieu penchés sur le concept de soi. Selon Hamel (2001), l'estime de soi revêt un caractère évaluatif faisant référence à l'appréciation subjective du concept de soi. L'Écuyer (1994) mentionne que le terme « estime de soi » était utilisé par Coopersmith (1967) et Ziller (1973) au lieu du terme « concept de soi ». Selon l'Écuyer (1994), le terme estime de soi se définit comme un concept unique ne possédant qu'une dimension, tandis que le concept de soi revêt plusieurs dimensions, ayant la connotation d'un système multidimensionnel complexe (Kraft, 1999). Le modèle multidimensionnel et hiérarchique du concept de soi, élaboré par Shavelson, Hubner et Stanton (1976), schématise ces multiples dimensions (annexe 2). Tantôt, cette notion réfère au concept de soi global (ou non scolaire), lequel 
comprend les dimensions émotionnelle, sociale et physique, tantôt, elle réfère au concept de soi scolaire qui inclut des niveaux spécifiques selon les matières scolaires (mathématique, français, anglais, sciences).

Le concept de soi est l'élément central de la personnalité d'un individu (Herry \& Worth Gavin, 1996) et Rosenberg (1979) le considère comme un cadre de référence fondamental (Famose, 2001); ce déterminant est complexe et varie (Gueyaud \& Dassa, 1998) selon le degré de motivation de l'individu. Byrne et Shavelson (1986) soulèvent que la notion de concept de soi spécifique ne doit en aucun cas être évacuée en l'incorporant à un concept de soi global, son évaluation demande donc une grande rigueur. Toutefois, il est clair, selon plusieurs auteurs (Famose, 2001; Ayotte, 1995; Hamel, 2001), que c'est au fil des expériences, des comportements posés par l'individu (attributions causales) et des interactions avec l'environnement que s'acquièrent les perceptions consolidant ces différentes facettes du soi, dont l'estime de soi. Étant donné la complexité du concept de soi, nous préférerons observer, au cours de la présente étude, les manifestations de l'estime de soi au travers de l'évolution des compétences personnelles et sociales.

\subsubsection{La dimension physique du concept de soi et l'estime de soi}

Nous n'approfondirons pas chaque dimension du concept de soi, mais nous tenons à mentionner que des études récentes ont conféré au concept de soi physique neuf facettes : la force, la corpulence (embonpoint), l'activité physique, l'endurance, 
la compétence sportive, la coordination, la santé, l'apparence physique et la souplesse (Marsh et al., 1994 tiré de Famose, 2001). Famose (2001) estime que les deux aspects majeurs composant le concept de soi physique sont le concept de soi physique global et l'estime de soi. Les sentiments positifs développés à l'égard de son propre soi physique (estime de soi) se rapportent à l'apparence corporelle, à l'image de soi ou au développement d'habiletés physiques.

De leur côté, Brassard et Mongeau (2004) soutiennent que l'image corporelle s'acquière chez le jeune au cours de son développement et à travers ses relations sociales. Les interactions sociales sont donc fondamentales au développement du soi. D'ailleurs, le guide ${ }^{6}$ École en santé (Martin \& Arcand, 2005) inclut les compétences sociales parmi les facteurs-clés du développement du jeune au même titre que l'estime de soi.

Brassard et Mongeau (2004) identifient l'image de soi comme étant directement en lien avec le développement des attitudes et comportements alimentaires déviants. En outre, Famose (2001) insiste sur l'importance de développer une perception positive à l'égard des habiletés physiques de l'individu afin de favoriser la pratique régulière de l'activité physique. Très intimement liées à l'image corporelle (apparence physique), les habiletés physiques (compétences) se

${ }^{6}$ Préparé par un comité national d'orientation composé de représentants de l'Institut national de santé publique du Québec (INSPQ), du ministère de 1'Éducation, du Loisir et du Sport (MELS), du ministère de la Santé et des Services sociaux (MSSS), de l'Association québécoise d'établissements de santé et de services sociaux (AQESSS) et de la Table de concertation nationale en promotion et en prévention (TCNPP). 
développent conjointement sans pour autant suivre la même progression. Ainsi, lors d'une intervention expérimentale axée sur un programme d'entraînement à la condition physique, Marsh et Peart (1998, tiré de Famose, 2001) révèlent que les activités orientées vers la coopération sont plus bénéfiques que les activités orientées vers la comparaison sociale, c'est-à-dire lorsqu'il y a des gagnants et des perdants. Ce constat explique en partie pourquoi plusieurs programmes expérimentaux de plein air, favorisant la coopération, améliorent le concept de soi (estime) des participants (Neill \& Richard, 1998; Neill, 2000; Hattie et al., 1997; Ann Klint, 1999). Comme il sera défini dans les prochaines sections, une séquence d'aventure propose ainsi plusieurs activités susceptibles de faire émerger la coopération.

\subsubsection{Les compétences sociales (le soi social)}

Les compétences sociales représentent la capacité d'un jeune à maîtriser un certain nombre d'habiletés sociales, cognitives et affectives lui permettant de vivre des relations sociales de qualité (Bowen et al., 2001, tiré de Martin \& Arcand, 2005). Hamel (2001), tout comme Duclos (2001), affirme que le soutien social provenant en particulier des parents et des amis contribue à rehausser l'estime de soi par la fonction dite émotionnelle qui correspond à toute expression d'appréciation et de valorisation envers l'enfant et l'adolescent. Le soutien social de la famille influence le concept de soi social relatif à la famille, tandis que le soutien social des pairs agit sur le concept de soi relatif aux pairs (Bolognini et Plancherel, 1998, tiré de Hamel, 2001). Hamel (2001) souligne que le soutien social ainsi que l'environnement social 
favorisent la santé, et que l'apparence physique, les relations avec les pairs et les compétences physiques auraient une influence sur le soutien des pairs. Le développement des compétences sociales chez les jeunes rehausse leur estime de soi. Pour y parvenir, Martin \& Arcand (2005) considèrent l'importance de favoriser chez les jeunes la maîtrise de diverses habiletés. Ces habiletés sont identifiées dans la prochaine section.

\subsubsection{Une stratégie d'intervention afin d'orienter positivement l'estime de soi du jeune et les compétences sociales}

Duclos (2000), Arcand et al. (1998) et Martin et Arcand (2005) sont d'avis qu'une intervention efficace en matière de santé doit agir simultanément sur l'individu et son environnement. Les actions déployées doivent notamment dégager un sentiment de confiance, d'appartenance et de réussite. Ces auteurs soulignent la connaissance de soi comme une composante fondamentale qui se traduit dans le PFEQ sous de multiples paliers, et notamment par le développement d'habiletés d'ordre personnel et social.

En 1998, le groupe interministériel sur les curriculums, volet Santé et bien-être (Arcand et $a l .1998$ ), établit un consensus autour de six habiletés qui sont redéfinies sous cinq appellations différentes par le MELS (Martin \& Arcand, 2005). Ces habiletés rendraient l'individu apte à maintenir une bonne santé : habileté à se fixer 
des buts, habileté à résoudre des problèmes, habileté à gérer son stress, habileté à communiquer et habileté à exercer son jugement critique.

Ces habiletés font référence à certaines compétences transversales, par exemple, celles d'ordre intellectuel (CT. 2 - résoudre des problèmes), d'ordre de la communication (CT. 9 - communiquer de façon appropriée), etc. Priest et Gass (1997) croient que pour développer harmonieusement ces habiletés chez l'enfant d'âge scolaire, ce dernier doit dépasser ses limites et se trouver dans un mode d'apprentissage psychosocial lui permettant de comprendre qu'il est unique et différent.

À ce point de la recherche, il semble clair que le mode d'intervention choisi doit favoriser la coopération entre les pairs, les rapports sociaux équitables entre les garçons et les filles, le développement d'habiletés physiques telles que l'endurance, la coordination, la souplesse, et explorer différentes connaissances et savoirs essentiels inscrits dans le PFEQ (2001) au troisième cycle du primaire.

Bien que les cadres de référence, les attributions causales, les évaluations d'autrui significatives, les expériences de maîtrise et la centralité psychologique (Famose, 2001) soient susceptibles d'affecter l'estime de soi ou le développement des compétences sociales, la présente étude explore principalement les habiletés d'ordre personnel et social en utilisant, comme outil d'intervention, l'aventure et le plein air. Neill et Richard (1998) soulignent que l'aventure éducative en plein air 
permet de vivre des situations d'apprentissage concrètes, de construire des relations harmonieuses et de rehausser l'estime de soi. Ces trois éléments sont maintenant soulignés dans le PFEQ (2001).

Hattie et al. (1997) soulignent qu'une activité développée judicieusement avec des intentions éducatives spécifiques et encadrée par un ou des individus compétents offre de plus grands bénéfices. Les jeunes se sentent ainsi en confiance et prennent plaisir à découvrir l'environnement dans lequel ils évoluent; en plus, cela les incite à utiliser des stratégies adéquates, par exemple, communiquer entre eux, afin de relever les défis qu'ils se sont donnés.

Pour y parvenir, nous avons utilisé la séquence d'aventure telle qu'elle a été établie par Robb et Ewert (1993, tiré de Bisson, 1999, p. 207) et adaptée par Bilodeau (2005a) en la jumelant à une séquence d'apprentissage.

\subsection{L'éducation par l'aventure en plein air : un pas vers la santé! Les retombées des modèles expérimentaux}

En 1998, Neill et son équipe publient une métaanalyse portant sur l'éducation en plein air. Les résultats démontrent que ce type d'intervention recèle un grand potentiel pour le développement humain tant sur les plans affectif, cognitif que physique (voir tableau 2.4.1), et gagne du terrain dans les domaines de la psychologie et de l'éducation. 
L'aventure éducative en plein air aurait un impact positif sur la santé des enfants et des adolescents et contribuerait à développer de saines habitudes de vie. Plusieurs chercheurs (Ann Klint, 1999; Hopkins \& Putnam, 1997; Neill \& Richard, 1998; Hattie et al., 1997) sont d'avis que l'éducation par l'aventure engendre des impacts positifs sur le concept de soi, le leadership, les relations interpersonnelles et la personnalité du jeune qui y participe.

Dans le livre Adventure Programming, Ann Klint (1999) explique que l'aventure en plein air influencerait le concept de soi, la réalisation « de sens », la motivation et aussi la gestion du stress. Charbonneau (2001) ajoute que, vécu à l'intérieur du cadre scolaire, ce type d'activités favorise l'accomplissement et les rencontres de soi, des autres et de l'environnement.

Dans le même ordre d'idées, plusieurs recherches sur l'aventure ou l'éducation en plein air effectuées à travers le monde (Gass, 1993; Hattie et al., 1997; Neill, 2000) suggèrent que ce type d'activités améliore les relations interpersonnelles, la coopération et la communication, et développe l'esprit d'initiative, tout en favorisant les apprentissages disciplinaires. À la suite de l'analyse de 43 études portant sur des programmes d'aventures éducatives, l'équipe de Neill (1998) conclut que ce genre de programme, c'est-à-dire l'éducation ou l'aventure en plein air (outdoor education ou adventure education) et l'apprentissage expérientiel (experiential learning), conduit généralement vers une perception positive des participants à leur égard. 
Le tableau 2.4.1 présente un résumé des impacts perçus lors de l'évaluation de modèles expérimentaux. Pour chaque catégorie, les auteurs de l'étude ont mis en lumière la grandeur des effets à la suite de l'expérimentation et de la méthodologie des programmes examinés. Ils ont (Neill \& Richard, 1998) noté des effets plus marqués pour la catégorie du concept de soi et ce, à long comme à court terme.

Tableau 2.4.1 : Résumé des impacts perçus lors d'évaluations de modèles expérimentaux d'aventure éducative en plein air?

\begin{tabular}{|l|l|}
\hline Catégorie & Description \\
\hline Leadership & $\begin{array}{r}\text { Conscience, prise de décision, leadership, capacité organisationnelle, gestion } \\
\text { du temps, valeur, buts. }\end{array}$ \\
\hline Concept de soi & $\begin{array}{r}\text { Capacités physiques/apparence, relation avec les pairs, général, académique, } \\
\text { confiance, efficacité personnelle, famille, compréhension de soi, bien-être, } \\
\text { indépendance. }\end{array}$ \\
\hline Scolaire & $\begin{array}{c}\text { Mathématique, lecture, niveau supérieur à la moyenne, résolution de } \\
\text { problèmes. }\end{array}$ \\
\hline Personnalité & $\begin{array}{c}\text { Féminin/masculin, accomplissement, motivation, stabilité émotionnelle, } \\
\text { agressivité, affirmation, « locus of control », maturité, réduction de la } \\
\text { nervosité. }\end{array}$ \\
\hline Relation interpersonnelle & $\begin{array}{c}\text { Coopération, communication interpersonnelle, compétences sociales, } \\
\text { comportement, habiletés connexes. }\end{array}$ \\
\hline Aventure & Défis personnels, flexibilité, conscience environnementale, forme physique. \\
\hline
\end{tabular}

Comme il a été mis en lumière précédemment, l'éducation par l'aventure et le plein air présente des atouts intéressants comme mode d'intervention en matière d'éducation à la santé. Ce mode d'intervention éducative offre l'occasion d'identifier et de résoudre des problèmes réels de la vie et ce, dans un climat riche en stimulations. Afin d'établir clairement la ligne de conduite à suivre lors du

\footnotetext{
${ }^{7}$ Tableau inspiré de Neill et Richard (1998) : Table 1. Effect sizes (ESs) reported for outcome measures.
} 
développement de la stratégie (mode) d'intervention, nous explorerons, dans les prochaines sections, les assises théoriques de l'approche utilisant le plein air.

\subsubsection{L'éducation en plein air (outdoor education)}

Au début des années 1960, Smith (1963) estime qu'apprendre en plein air représente un prolongement et un enrichissement du curriculum scolaire à travers les

expériences vécues. À l'époque, l'éducation en plein air se définit comme une façon d'effectuer des apprentissages disciplinaires au cœur d'un laboratoire naturel. En 1977, Grenier définit l'éducation en plein air comme une éducation expérientielle, sensorielle, émotive et vécue. Quelques années plus tard, Bilodeau (1980) rapporte que le rythme de la vie moderne, dans lequel la rapidité et la performance sont « rois », engendre la détérioration accélérée de l'environnement naturel par la surexploitation ou la mauvaise gestion des ressources naturelles. Il ajoute que les individus consacrent plus de temps au travail et diminuent le temps accordé à la pratique de l'activité physique. Ford (1986) poursuit dans la même veine et décrit l'expression « éducation en plein air » comme un ensemble d'activités de loisirs véhiculant des valeurs. Il ajoute dans sa définition l'omniprésence de liens entre les interactions sociales et le respect des ressources naturelles, qu'il qualifie comme l'élément fondamental du troisième millénaire. Hopkins et son équipe (1997) décrivent l'éducation en plein air comme une approche organisée à travers laquelle 
les apprentissages peuvent faire différer le cheminement des individus et ce, directement au cœur de l'expérience.

Ainsi, Suzuki (2001) et Gass (1993) considèrent que l'humain désire accroître ses aptitudes mentales, physiques et spirituelles dans le but d'améliorer sa compréhension du monde écologique, explorer sa créativité, favoriser son ouverture d'esprit pour enrichir son équilibre spirituel. Gass (1993) croit que l'aventure facilite le cheminement de l'individu dans ce sens.

\subsubsection{L'éducation par l'aventure (adventure education)}

L'aventure se définit comme : « ce qui arrive d'imprévu, de surprenant » (Petit Robert, 1990). C'est l'éducation en plein air abordée sous l'angle du cheminement personnel, intrapersonnel et interpersonnel. Raiola et al. (1999) qualifient l'aventure en soi comme un besoin humain : «L'aventure c'est beaucoup plus qu'un mot, c'est une atmosphère, une attitude, un état d'esprit, concluent-ils, c'est la curiosité de voir de l'autre côté de la montagne! ") Bien qu'il n'y ait pas de consensus entre les chercheurs et les praticiens sur la question (Raiola et al., 1999; Priest et Gass, 1997) tous s'entendent pour dire que l'aventure comporte un élément d'excitation, d'incertitude, d'effort, de risque réel ou perçu et d'interactions avec l'environnement naturel. Le concept d'aventure s'apparente en tous points avec le concept d'éducation en plein air, mais comprend un élément supplémentaire particulièrement important : la notion de risque (Gass, 1993). Cette notion implique que l'individu qui 
participe à des activités dites d'aventure sera appelé à sortir de sa zone de confort, à dépasser ses limites, à explorer un monde encore inconnu (figure 2.4.1). Le risque comporte le potentiel de perdre quelque chose, que ce soit sur les plans physiques (se casser un bras), sur le plan psychologique (avoir peur) ou sur le plan social (être embarrassé par la présence des pairs). Le risque est créé par la présence du danger. Les activités d'aventure de groupe ou individuelles comportent une tâche complexe qui mène l'individu au dépassement et à l'accomplissement.

Par exemple, lors d'activités dites d'aventure en plein air, le participant rencontre l'inconnu, l'inconfort. Il doit alors cheminer dans des situations où ses connaissances, ses compétences et ses schèmes de références (famille ou autres), sont dépassés et ses repères habituels parfois absents. Ainsi, pour parvenir à réaliser le défi, il doit s'adapter à ces nouvelles situations en utilisant ses compétences.

Lorsque l'individu sort de sa zone de confort, il rencontre des situations problématiques pour lesquelles il doit rapidement trouver des solutions appropriées. Il va de soi qu'il doit exploiter son habileté à résoudre des problèmes. Gass (1993) soulève que le concept d'aventure en plein air est de plus en plus utilisé à des fins thérapeutiques. L'auteur note que son utilisation provoquerait une métamorphose des fonctions cognitives et émotionnelles. La figure 2.4.1 «Dépasser ses limites pour évoluer » présente les choix potentiels qu'un individu doit effectuer lorsqu'il participe à une activité dite d'aventure. Nadler (1993) croit que le fait, pour un individu, d'être exposé à des expériences extérieures à sa zone de confort, a un 
impact positif sur son développement global. Toutefois, celui-ci doit pouvoir se réfugier dans sa zone de confort, si cela est nécessaire. Ce principe rejoint la théorie de Maslow (point 2.1.1), qui souligne que la zone de confort est ce lieu sécuritaire dans lequel l'individu se réfugie une fois ses besoins de base comblés afin de mieux évoluer avec autrui (socialiser) et éventuellement accroître son estime personnelle. Bilodeau $(2000,2005 b)$ explique qu'en activité d'aventure en plein air chaque individu possède trois zones de confort: la zone de confort environnementale (nature), la zone de confort humain et la zone de confort de l'activité d'aventure en question. Les trois zones méritent d'être développées simultanément pour que l'individu ressente un bien-être dans la nature, avec les autres et dans l'activité proposée. Bilodeau $(2000,2005 b)$ mentionne que : « sortir de cette zone de confort crée un inconfort qui éveille les sens, éveille son soi et l'importance de sa présence dans un groupe ». Cette zone d'inconfort provoque l'incertitude, mais de retour dans la zone de confort, l'individu développe le bien-être et la confiance de vivre. Cette évolution se réalise lorsque l'individu réussit (ou accomplit) les défis qui lui sont présentés. 


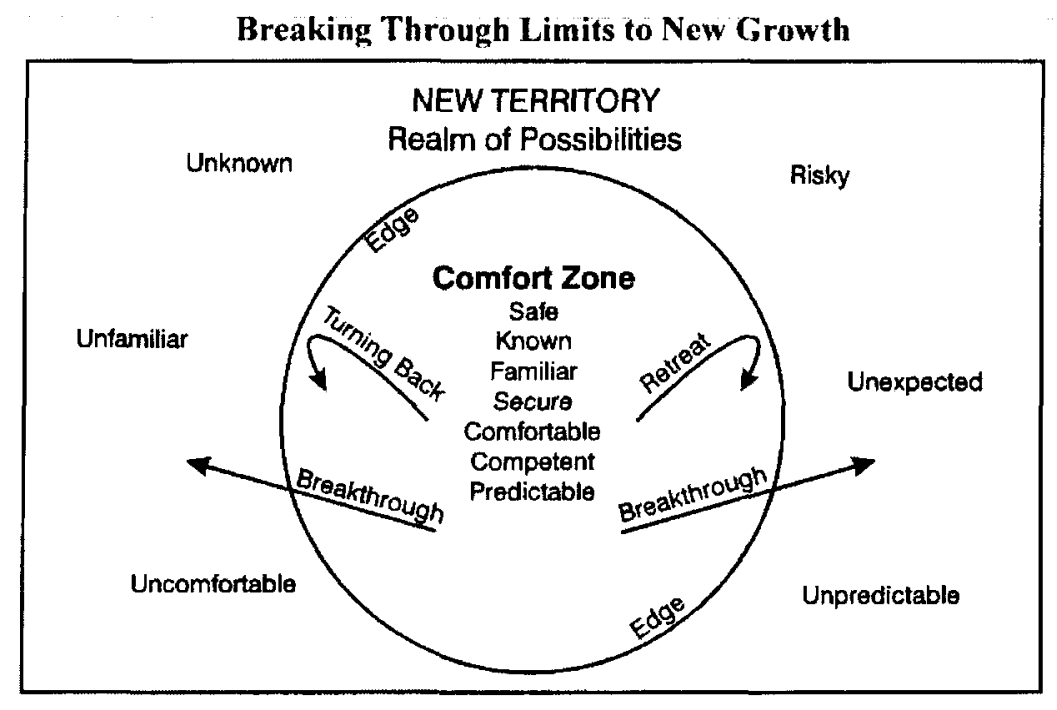

Figure 2.4.1 : «Dépasser ses limites pour évoluer ${ }^{8}$

Voguer entre le risque, l'inconnu et la sécurité conduit généralement vers le développement personnel. Hattie et al. (1997) et Gass (1993) estiment que le concept d'aventure en plein air favorise le développement de la personne sur plusieurs plans : scolaire, personnel, concept de soi, relations interpersonnelles, etc. Toutefois, il ne suffit pas de proposer (ou de pratiquer) une activité en plein air, et encore moins de s'y aventurer à l'aveuglette, pour atteindre un accomplissement personnel. Les auteurs (Kraft, 1999; Gass, 1993) sont clairs : l'orientation des actions et leur planification sont primordiales (programming) pour favoriser le cheminement des participants et ce, au même titre que le milieu scolaire l'exige. Neill et Richard (1998) considèrent que l'un des éléments les plus importants de l'aventure en plein air demeure la motivation. L'individu qui y participe doit y trouver un intérêt. L'expérience doit tenir compte du potentiel, des compétences et des capacités des

\footnotetext{
${ }^{8}$ Nadler (1993, p.58)
} 
individus qui la réalisent (Neill \& Richard, 1998 et Hopkins \& Putnam, 1997). De là l'importance de la planification et de l'évolution des situations d'apprentissage par l'aventure.

\subsubsection{L'apprentissage expérientiel en plein air (experientiel learning)}

Le meilleur moyen d'apprendre, selon Priest et Gass (1997), c'est d'être en contact avec l'apprentissage. Autrement dit, apprendre par l'expérience, être actif physiquement, avoir des stimulations cognitives significatives et être émotionnellement engagé.

En 1939, Dewey (Rabiola, \& O’Keefe 1999) croyait que les apprentissages s'effectuaient directement au cœur de l'expérience et que celle-ci influençait l'ensemble du développement humain sur les plans physique, social et émotionnel. Ce type d'expérience requiert de l'apprenant qu'il accepte la responsabilité de ses propres actions et qu'il soit disposé, à la suite de son expérience, à entreprendre une réflexion. L'apprentissage expérientiel combine un processus actif (expérience) à un moment passif (réflexion). Le processus d'apprentissage expérientiel, selon Joplin (2008), est généralement cyclique et se vit en cinq étapes. La première étape, le focus, inclut la présentation de la tâche en isolant l'attention de l'apprenant. En d'autres mots, l'intervenant définit le sujet étudié et présente à l'élève le défi à relever. La deuxième étape, l'action, place l'apprenant en situation de stress, car l'activité proposée exige de nouvelles connaissances ou habiletés. L'action peut être 
physique, mentale, émotionnelle, spirituelle ou toucher une autre dimension, et doit imputer la responsabilité à l'apprenant. La troisième étape, le support, se vit au cours de l'expérience à différents degrés. Le soutien assure à l'apprenant un minimum de stimulations et d'informations qui lui permettent de relever le défi demandé. Ce soutien est verbal, physique ou écrit. La rétroaction, quatrième étape du processus, comporte principalement l'adresse de commentaires constructifs à l'égard du travail accompli par l'apprenant. Cette rétroaction s'applique notamment en rapport avec la nature du travail accompli et les interactions sociales observées. Finalement, le compte rendu verbal aide l'apprenant à rendre compte véritablement de la situation, car il doit être capable de reconnaître, articuler et évaluer la situation vécue. Cette cinquième étape peut s'actualiser sous forme de discussion de groupe, de présentation ou de projet classe. Ce compte rendu verbal contribue à favoriser chez l'apprenant une juste perception de la situation. Joplin (2008) conclut que l'apprentissage expérientiel nécessite l'implication active de l'intervenant et de l'apprenant dans la situation.

Également, la littérature rapporte que l'évolution des schèmes de référence se modifie lors de situations dissonantes (Horwood, 1999; Sandrin-Berthon, 1997; Houssaye, 1994), par exemple, lors de l'inconfort du moment présent ou le désir futur d'une situation confortable (homéostasie corporelle). En présentant des situations de dissonance, l'individu évolue avec ses pairs dans la recherche de solutions. L'apprentissage par l'expérience est un processus d'apprentissage qui se construit et se modifie selon les circonstances (Priest \& Gass, 1997). 
Pour bien comprendre le potentiel de l'éducation par l'aventure et le plein air en milieu scolaire, nous établirons, au point 2.5.1, un survol des liens existant entre les philosophies du plein air et les théories de l'éducation.

\subsection{Les modèles expérimentaux en lien avec les théories de l'éducation}

Les modèles expérimentaux d'éducation en plein air s'effectuant au niveau du primaire misent généralement sur trois principes ${ }^{9}$ (Hopkins \& Putnam, 1997) :

1. Construire chez les jeunes une curiosité et un intérêt naturels.

2. Utiliser les avantages de l'environnement immédiat de l'école pour développer les activités d'aventure et la proximité.

3. Poursuivre l'expérience en classe et ensuite auprès des parents et dans la communauté afin de soutenir les activités (soutien de l'environnement social, volet de l'approche écologique).

L'éducation par l'aventure en plein air utilise les concepts d'éveil et de curiosité, concepts mis de l'avant par Bruner (1963). Bruner rapporte que l'éveil et la curiosité sont essentiels au développement de compétences et à toutes formes d'apprentissage. En outre, ce type d'approche propose des activités de confiance et de communication qui suscitent chez les participants un partage d'informations menant à des apprentissages sociocognitifs (Vygotski, 1985). L'organisation

${ }^{9}$ Traduction libre de Adventure Education In The Primary Phase Cleves Country Middle School (Case Study 6.1). 
d'activités communes, au travers desquelles les apprenants effectuent des échanges sociaux, devient une source de développement conceptuel de l'enfant. L'éducation par l'aventure en plein air suggère l'appropriation de l'environnement (culturel ou autre). Les auteurs soutiennent que les apprentissages émergent d'un processus social et interpersonnel lorsque l'enfant est actif à travers son environnement (Priest \& Grass, 1997; Miles, 1999).

Garnier, Bednarz et Ulanovskaya (1991) soulèvent que des chercheurs en didactique fondent le développement des dynamiques individuelles sur l'expérience sociale. Ces dynamiques interviennent à titre de premier constituant; toutefois, loin de considérer les interactions sociales comme le seul moteur de ce développement, il faut davantage saisir le processus d'apprentissage au fil des actions et des interactions.

L'éducation par l'aventure et le plein air est une méthode active qui favorise la résolution de problèmes (Priest \& Gass, 1997) et la prise de risques (Gass, 1993; Priest \& Gass, 1997). Cela permettrait à l'enfant, selon Turcotte (2006) de développer plusieurs des facteurs clés en matière de santé dont l'estime de soi, les compétences sociales, en plus d'offrir un terreau riche à l'actualisation disciplinaire. 


\subsubsection{Un survol des liens existants entre l'aventure éducative en plein air et les} théories de l'apprentissage

Dewey (Houssaye, 1994), grand philosophe américain des $\mathrm{XIX}^{\mathrm{e}}$ et $\mathrm{XXe}$ siècles, croit que les connaissances doivent toujours s'enraciner dans l'expérience et l'action de la personne. Il affirme que l'éducation doit se situer dans l'environnement naturel de l'être humain.

Pour soutenir l'intérêt et la motivation de l'apprenant, Dewey (Houssaye, 1994) suggère, entre autres, de rallier les dualismes traditionnels tels que la raison et le corps, le corps et l'âme, l'esprit et l'action, le psychologique et le social, l'individu et la société, l'élève et le curriculum, la théorie et la pratique, le travail et le loisir, l'activité pratique et l'activité intellectuelle ainsi que l'humain et la nature. Ces principes se situent à la base de l'éducation en plein air (Henton, 1996; Smith, 1963; Priest \& Gass, 1997). L'aventure éducative en plein air rallie ces dualismes et, à travers les défis, amène l'individu à les confronter. Grass (1993) mentionne que l'apprenant assimile davantage les apprentissages s'il y participe que lorsqu'il les reçoit comme spectateur. Aussi, l'auteur croit que l'aventure éducative en plein air permet d'explorer et d'étudier l'espace afin de mieux vivre les apprentissages. À titre d'exemple, De Koninck (2004) mentionne que l'éducation du coureur des bois ou du marin en mer est supérieure dans l'agir humain puisque leur sens sont aguerris et leur conscience sensorielle (awareness) est toujours plus éveillée. 
Par ailleurs, Vygotski (1985) considère que l'apprenant se développe grâce aux moyens qu'il trouve dans son environnement social et grâce à la multitude d'interactions sociales. La distance entre le niveau de développement actuel, ce que l'enfant peut apprendre seul, et le niveau de développement potentiel, ce que l'enfant peut apprendre avec l'aide de plus qualifié que lui, se nomme la zone proximale de développement. Dans les activités d'aventure éducative en plein air, la nature sociale des apprentissages (Vygotski, 1978, tiré de Henton, 1996) est vécue sous un angle différent. Car, dans ce type d'activité, lorsque le conflit prend place, les individus en cause doivent rapidement trouver une solution puisque généralement la réalisation de l'activité en dépend.

Le PFEQ propose une formation par compétences qui comporte l'organisation et l'intégration de plusieurs principes issus du cognitivisme et du constructivisme (PFEQ, 2001). Tout d'abord, le cognitivisme, parce qu'il rend compte des processus qui permettent à un individu d'intégrer de nouveaux savoirs à son système de connaissances et de les utiliser dans de nouveaux contextes. La théorie du cognitivisme soutient que l'apprenant s'adapte aux différentes situations en assimilant la réalité extérieure et en la modifiant selon ses schèmes de référence (Lasnier, 2000); toutefois, l'objet d'apprentissage, pour susciter l'intérêt de l'élève, doit être suffisamment complexe, sans être abstrait. Bruner (1963) reconnaît aussi le besoin des jeunes d'effectuer un apprentissage actif qui favorise les découvertes et les apprentissages personnels. 
Ensuite, le constructivisme, parce qu'il conçoit la connaissance comme la résultante des actions, d'abord réelles, puis intériorisées, de l'individu sur les objets, sur leur représentation ou sur des propositions abstraites. Tandis que le socioconstructivisme souligne la nature foncièrement sociale de la pensée et de l'apprentissage, les concepts étant des outils sociaux qui soutiennent l'échange de points de vue et la négociation de significations (Dontigny \& Roy, 2007).

Ainsi, le développement de compétences se manifeste dans des contextes d'une certaine complexité et son degré de maîtrise peut progresser tout au long du parcours scolaire et même au-delà, tout au long de la vie (PFEQ, 2001). Pour être à leur maximum les apprentissages sont effectués suivant une hiérarchie, sous forme de situations où se développe et se manifeste le savoir-agir.

La figure 2.5.1 présente la structure d'une tâche en conformité avec la formation par compétences. Lors de la réalisation d'un projet, la mobilisation requise des ressources implique que la personne compétente tienne compte des contraintes issues du contexte et ajuste son agir (PFEQ, 2001). Cette approche permet d'acquérir les connaissances et habiletés nécessaires au développement de compétences transversales et disciplinaires (Lasnier, 2000; Gagné, Belley, Legault \& Samson, 2003). Par conséquent, pour que les élèves réalisent un projet, les titulaires de classe effectuent des activités d'apprentissage spécifiques des domaines d'apprentissage ciblés, et proposent ensuite des tâches plus complexes en lien avec la réalisation du projet. Le projet, d'une durée indéterminée, peut être réalisé par un groupe-classe ou 
par plusieurs groupes-classes, d'un même cycle ou de cycles différents tandis qu'une tâche est : «une situation d'apprentissage contextualisée et complexe qui s'inscrit dans un domaine général de formation et qui permet aux élèves d'activer dans l'action une compétence transversale et une compétence disciplinaire " (Gagné et al. 2003, p.8).

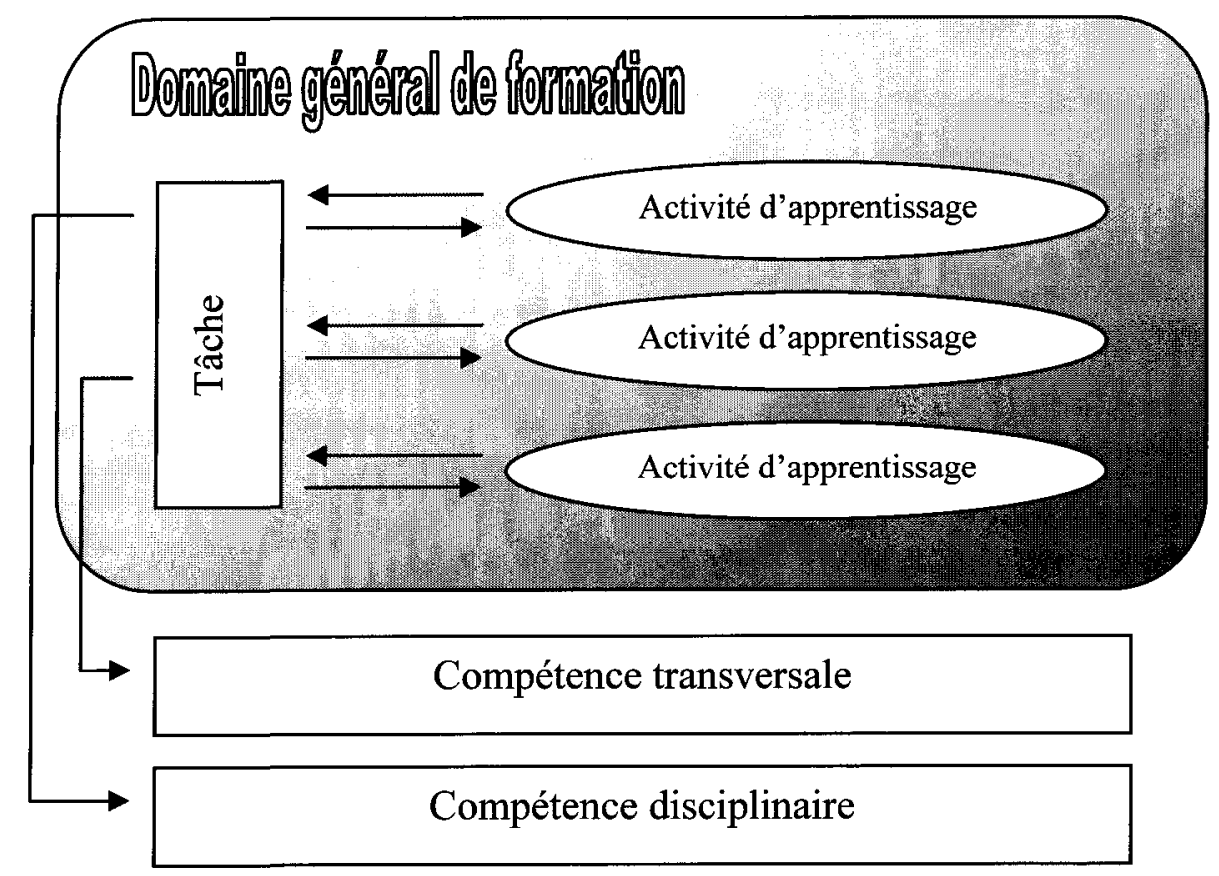

Figure 2.5.1 : Illustration de la structure d'une tâche en conformité avec la formation par compétences ${ }^{10}$

En admettant que la séquence d'aventure et d'apprentissage met l'accent sur la dimension relationnelle de l'apprentissage, l'aspect socioconstructiviste issu du constructivisme se bonifie. La dimension de contact avec les autres permet de construire les connaissances qui serviront lors d'apprentissages futurs, si, bien sûr, la

${ }^{10}$ Gagné, C. et coll. (2003, p. 8) 
notion de transferts s'opère (Burner, 1960; tiré de Gass, 1999). La séquence d'aventure intégrée à une séquence d'apprentissage doit permettre d'organiser et de regrouper de façon cohérente des tâches intégratrices et des activités d'apprentissage. Cette approche viserait l'intégration d'un ensemble de compétences et l'appropriation de contenus disciplinaires ayant un caractère commun (Lasnier, 2000, p. 211) et utiliserait, comme le suggère le PFEQ, les concepts de cognitivisme et de constructivisme.

\subsubsection{Intégrer la séquence d'aventure à la séquence d'apprentissage}

Les participants devraient expérimenter des méthodes, des notions et des concepts imaginés ou appris selon une séquence d'aventure (et d'apprentissage) prédéterminée. Le modèle original de la séquence d'aventure (Robb et Ewert (1993), tiré de Bisson, 1999, p.207) adapté par Bilodeau (2005a) propose une évolution des défis éducatifs en plein air en sept étapes (figure 2.5.2). Les premières étapes visent l'approche sensorielle et l'acclimatation (Bilodeau, 2005a), les activités de connaissance et les activités de désinhibition. Ces activités sont utiles pour préparer les participants à vivre des activités plus complexes de communication, de coopération, de confiance et de résolution de problèmes en situation d'aventure.

Comme le suggère Freinet (Boumard, 1996) dans sa méthode naturelle ou de tâtonnement expérimental, le principe se fonde aussi sur l'idée de la non-dissociation de l'affect et de l'intellect dans les apprentissages, auxquels les notions de réussite et 
de plaisir sont associées (Houssaye, 1994). Cette méthode naturelle d'apprentissage proposée par Freinet représente un tremplin vers les apprentissages significatifs réalisés en plein air. De plus, Bilodeau (2005a) met de l'avant à la figure 2.5.2., une période de réflexion, suivie d'une cérémonie de partage et d'une célébration dans un contexte d'accompagnement par les intervenants, qu'il qualifie de période de transfert. Par ailleurs, Gass (1993) ajoute que pour cheminer à travers les défis proposés dans l'éducation par l'aventure, le jeune doit utiliser l'ensemble de ses capacités personnelles sur les plans physique, cognitif, et social.

L'éducation par l'aventure en plein air, tout comme le recommande Dewey (Houssaye, 1994) et le suggèrent les théoriciens en promotion de la santé (Naidoo \& Wills, 1994; Turcotte, 2006), contextualise les apprentissages. Ce type d'intervention opte pour la continuité entre la raison et le corps, l'individu et la société, la personne et la nature.

Breton et al. (2002) suggèrent que l'environnement dans lequel le jeune évolue ainsi que les individus qu'il côtoie jouent un rôle important dans le développement de son estime et le développement de compétences qui s'effectuent généralement à travers un ensemble de situations comparables.

Dans cette optique, la progression de la séquence d'aventure qui propose des défis en milieu naturel revêt un intérêt particulier, car, tout comme le recommande le PFEQ (2001), il semble primordial de graduer le niveau de complexité en vue d'assurer un développement continu et progressif des compétences. La séquence 
d'aventure présentée à la figure 2.5.2 (Bilodeau, 2005a) s'amorce par des activités d'approche sensorielle et d'acclimatation qui encouragent la connaissance de soi, dans ce nouvel environnement physique et parfois social. Des activités de connaissance qui facilitent les échanges entre les participants viennent par la suite. Les activités de déshinibition contribuent à tisser des liens entre les participants dans un esprit ludique, ce qui les prépare à des activités plus complexes de communication, coopération et confiance mutuelle. L'évolution de la séquence accorde une importance particulière aux relations interpersonnelles, pour se terminer par des défis d'ordre personnel et groupal comme le recommandent certains théoriciens en matière de santé (Mérini, Jourdan, Victor, Berger \& De Peretti, 2004; Turcotte, 2006). La séquence d'aventure suggère l'évolution des défis passant de l'acclimatation des participants au milieu naturel, à l'expédition proprement dite. Afin d'assurer le développement de l'estime personnelle des participants, le caractère plus ou moins familier de certaines tâches (Dontigny \& Roy, 2007) et la complexité des problématiques interpersonnelles rencontrées sont au cœur de l'évolution de la séquence d'aventure. Plus précisément, l'enchaînement des activités doit être adapté au groupe. À ce sujet, Gass (1993) et Hirsch (1999) soulignent que le cheminement dans un groupe provoque des situations socialement significatives (conflit sociocognitif) et s'inscrit dans un processus où l'action et les interactions sociales sont intimement liées.

Mettre en place, au cœur du curriculum scolaire, un projet d'éducation à la santé par l'aventure et le plein air demande la collaboration de la direction, des 
enseignants et des parents. Des liens étroits coexistent entre les activités d'apprentissage, les tâches intégratrices et la séquence d'aventure pour conduire le groupe vers la réalisation d'un projet commun.

En s'enrichissant et en se nourrissant mutuellement, la séquence d'aventure et la séquence d'apprentissage formeraient un contexte favorisant le développement d'une santé globale, notamment par l'amélioration des compétences personnelles et sociales des jeunes et le rehaussement de leur estime. 


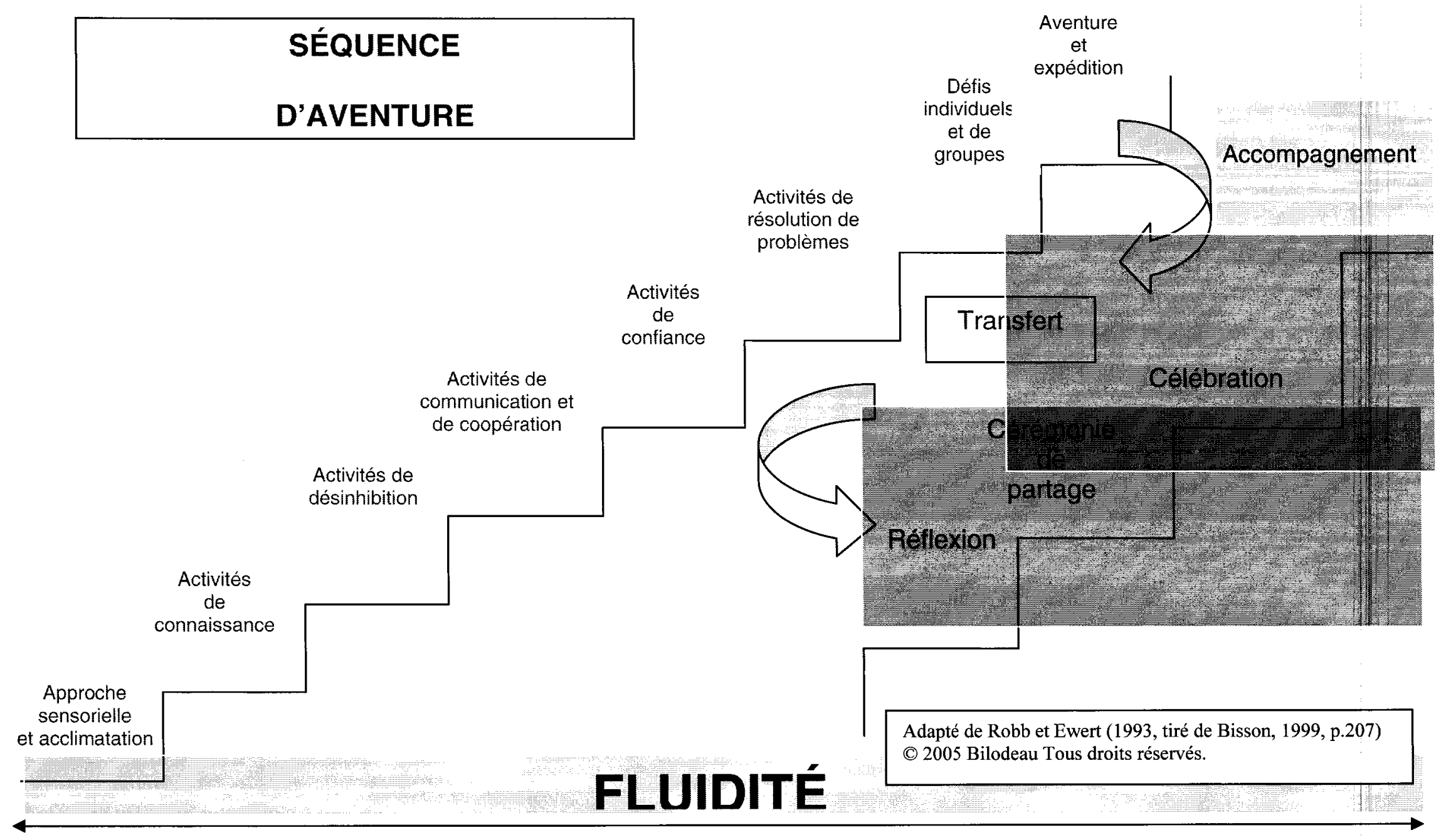

Figure 2.5.2 : Séquence d'aventure 


\section{CHAPITRE 3}

\section{Méthodologie}

Cette recherche utilise un cadre épistémologique naturaliste qui illustre une recherche-action de type pragmatico-interprétatif (Savoie-Zajc, 2001). La conception d'une stratégie d'intervention active en promotion de la santé, au cœur du système scolaire, produit plusieurs connaissances. L'analyse effectuée est de type qualitative/interprétative, en lien avec le domaine général de formation Santé et bienêtre et les compétences transversales d'ordre personnel et social ( 7 et 8), dévoilant le développement d'une conscientisation en matière de santé et la manifestation positive de l'estime de soi chez les jeunes. Finalement, un parallèle est établi avec certains savoirs essentiels de la troisième compétence (adopter un mode de vie sain et actif) du domaine d'apprentissage de l'éducation physique et à la santé. 


\subsection{Vue d'ensemble}

Le Programme de formation de l'école québécoise propose une véritable ouverture pour améliorer la santé et le bien-être des générations futures. Pour y parvenir, il semble indispensable (Turcotte, 2006; Motta, 1998) de passer par l'actualisation des disciplines scolaires. L'éducation par l'aventure recèle, selon plusieurs auteurs (Priset, 1997; Miles, 1999), d'innombrables occasions d'apprentissage. Ainsi, la présente recherche est une recherche-action qui porte sur l'intervention mise en place et effectuée en collégialité avec les acteurs du milieu scolaire, principalement deux titulaires du troisième cycle du primaire et l'intervenante (chercheure-acteure).

La recherche-action implique un processus cyclique d'action et de réflexion, qui s'inspire des études de Kurt Lewin (1946, dans Savoie-Zajc, 2001). La recherche-action propose alors de planifier l'action, d'agir et d'observer pendant l'action et, finalement, de réfléchir sur l'action (Savoie-Zajc, 2001). Cette pratique permet ainsi d'améliorer la conception des activités d'aventure et de les présenter sous forme de tâches intégratrices, menant à la réalisation du projet de classe.

Le premier défi de cette recherche réside dans la construction/conception d'un mode d'intervention efficace en matière de santé qui respecte les assises du renouveau pédagogique. Le deuxième défi est de favoriser, à travers la séquence 
d'aventure et d'apprentissage, l'émergence des facteurs de développement individuel favorables au maintien du bien-être et de la santé des participants et, finalement, le troisième défi est d'explorer les perceptions des intervenants sur les retombées potentielles en matière d'apprentissage scolaire.

\subsubsection{Les participants}

Les sujets fréquentent une école primaire pilote. Le groupe est composé de 28 jeunes, âgés de 10 à 12 ans. En tout, 17 garçons et 11 filles ont accepté de participer à la séquence d'aventure et d'apprentissage.

L'échantillonnage représente deux classes et compte l'ensemble des enfants inscrits au troisième cycle du primaire de cette école. La classe, composée majoritairement de $5^{\mathrm{e}}$ année, totalise 13 élèves, tandis que la classe composée uniquement de $6^{\mathrm{e}}$ année, compte 15 élèves. Il faut noter qu'en janvier, un jeune s'est joint au groupe et, lorsque pertinents, ses commentaires ou les propos des intervenants scolaires à son endroit ont été retenus pour l'analyse des résultats.

Aussi, à la suite de la réalisation de chaque atelier, les deux titulaires de classe et, selon le cas, les autres intervenants associés à la réalisation des ateliers (enseignant d'éducation physique et à la santé, le psychoéducateur et autres) qui ont participés aux périodes d'autorégulation sous la forme de groupe de discussion avec la chercheure sont considérés comme des acteurs ayant participé au déroulement de 
cette recherche. Leurs commentaires ainsi relevés ont servi à consolider l'investigation de la séquence d'aventure et d'apprentissage en plus d'encourager la réflexion sur le processus de recherche.

\subsubsection{Mise en place de la recherche-action}

En juin 2003, les démarches ont été entreprises avec la direction de 1'école et les titulaires de classe du troisième cycle de l'école primaire sélectionnée.

La séquence d'aventure conçue s'appuie sur les assises du DGF Santé et bienêtre, vise le développement des compétences transversales d'ordre personnel et social, puis respecte les apprentissages (savoirs essentiels) et compétences à développer au troisième cycle du primaire. Le mode d'intervention proposé auprès des élèves est développé conjointement avec les titulaires de classe et respecte les visées du projet éducatif de l'école. Ainsi, l'implication des titulaires de classe dans la recherche favorise la cohérence, le respect et la confiance à l'égard de la démarche d'éducation à la santé empruntée (Savoie-Zajc, 2001).

La planification et la conception de la séquence d'aventure ont été actualisées autour d'éléments problématiques identifiés par les intervenants du milieu (SavoieZajc, 2001) et soutenus par les études exposant la situation en matière de santé chez les jeunes (voir chapitre 1, p. 6). Ainsi, les points d'ancrage utilisés pour la présente étude sont: la méconnaissance de l'environnement naturel; la diminution de la 
pratique d'activité physique chez les jeunes; et surtout la prévalence croissante des problèmes de santé de cette clientèle qui fait naître des difficultés d'apprentissage et d'adaptation sociale (Institut national de santé publique du Québec, 2004). En ce sens, les opinions des titulaires de classe et de l'enseignante d'éducation physique et à la santé ont été précieuses pour orienter les actions et assurer la continuité des apprentissages entre la séquence d'aventure et le cadre scolaire conventionnel.

Bien que la progression ait été prétablie, au moins deux ateliers à l'avance, quant au nombre d'ateliers et à leur durée, la poursuite de la séquence d'aventure était conditionnelle au bon fonctionnement des élèves et à la pertinence du contenu pour l'émergence ou la consolidation d'apprentissages.

À la suite de la réalisation de chaque atelier, il a été prévu de procéder à une période d'autorégulation sous la forme de groupe de discussion entre la chercheure et les participants impliqués. Dans le cadre de la présente recherche-action, les commentaires recueillis en cours d'expérimentation ont permis de procéder à un ajustement du contenu des ateliers suivants et ce, afin de mieux répondre aux besoins du milieu.

\subsection{La planification du programme d'aventure éducative en milieu scolaire}

L'éducation à la santé priorise des actions portant sur le développement des compétences personnelles et sociales, l'adoption de saines habitudes de vie et le 
rehaussement de l'estime de soi (Arcand et al., 1998; Martin \& Arcand, 2005; Tucotte, 2006). Le MELS suggère fortement que l'ensemble de l'équipe-école participe à la mise en place d'actions favorisant, chez les jeunes, l'adoption de comportements sains en matière de santé (PFEQ, 2001; Martin \& Arcand, 2005).

Dans cette optique, l'introduction d'une séquence d'aventure au PFEQ pourrait servir d'outil d'intervention, car elle sollicite la collaboration de plusieurs intervenants de l'équipe-école et peut permettre d'éviter la multiplicité des programmes de promotion de la santé (Arcand et al., 1998) en milieu scolaire.

Les documents, programmes et philosophies suivantes ont inspiré l'élaboration et la conception de la séquence d'aventure et d'apprentissage :

- Les modèles expérimentaux en plein air tirés de Hopkins et Putnam (1997);

- la séquence d'aventure éducative en plein air élaborée par Robb et Ewert (1993, tiré de Bisson, 1999, p. 207) et adaptée par Bilodeau (2005a) présentant l'évolution des défis éducatifs (figure 2.5.2);

- les assises du document élaboré par le groupe interministériel sur les curriculums, volet Santé et bien-être (Arcand et al., 1998) qui dévoilent les compétences essentielles liées à la santé et au bien-être à intégrer au curriculum des enfants de l'éducation préscolaire et des jeunes du primaire et du secondaire au Québec; 
- Le modèle théorique de la santé par l'action (HAM) (Tones \& Green, 2004). Figure 2.1.3.

Tous ces modèles mettent en lumière des éléments gagnants pour faciliter l'implantation d'un outil d'éducation à la santé en milieu scolaire. Dans son application, la séquence d'aventure et d'apprentissage utilise des méthodes qui rejoignent les théories du socioconstructivisme et du cognitivisme en offrant des situations d'apprentissage contextualisées.

\subsubsection{La synthèse des ateliers selon le mode d'intervention adopté}

Le tableau 3.2.1 présente une synthèse des ateliers d'aventure éducative en plein air suivis par les élèves du troisième cycle du primaire. Ce tableau offre une vue d'ensemble de la progression de la séquence d'aventure en lien avec le développement des habiletés ciblées pour maintenir une bonne santé. La première partie du tableau 3.2.1 présente la nature des activités à accomplir et les cases identifiées par un point noir tracent l'évolution du projet selon la séquence d'aventure adaptée par Bilodeau (2005a). La deuxième partie présente les cinq habiletés soulevées par le groupe interministériel sur les curriculums, volet Santé et bien-être (Arcand et al., 1998; Martin \& Arcand, 2005), et les cases sélectionnées indiquent le résultat attendu de ces habiletés. Nous tenons à mentionner que, même si certains termes sont parfois identiques, la nature des activités se différencie du 
processus menant au développement d'habiletés à développer pour maintenir une bonne santé.

La séquence d'aventure se définit comme étant un procédé, une méthode employée pour parvenir à un certain résultat, tandis que le développement d'habileté est un processus, c'est-à-dire une évolution dans le temps (Legendre, 2005). 
Tableau 3.2.1 : Ateliers d'aventure éducative en plein air suivis par les élèves du troisième cycle du primaire

\begin{tabular}{|l|l|l|l|l|l|l|l|l|l|l|}
\hline \multicolumn{7}{|l|}{ Ateliers 1 à 10 } & \multirow{2}{*}{ Projet } \\
\hline A1 & A2 & A3 & A4 & A5 & A6 & A7 & A8 & A9 & A10 & \\
\hline
\end{tabular}

\begin{tabular}{|c|c|c|c|c|c|c|c|c|c|c|c|}
\hline \multicolumn{12}{|c|}{ Séquence d'aventure (Bilodeau, 2005a; adaptation de Robb et Ewert (1993, tiré de Bisson, 1999, p.207 } \\
\hline Approche sensorielle et acclimatation & - & & - & & & & & - & & & \\
\hline Activité de connaissance & - & & & & & & & & & & \\
\hline Activité de désinhibition & & - & - & - & & & & & & & \\
\hline Activité de communication et de coopération & - & - & च & & " & " & - & & $\square$ & & \\
\hline Activité de confiance & & & a & & घ & " & च & & " & घ & \\
\hline Activité de résolution de problèmes & & - & & & - & & - & $\square$ & घ & च & \\
\hline Défis individuels et de groupe & & & घ & च & & - & & I. & घ & घ & \\
\hline Aventure et expédition & & & & - & & & & - & " & च & \\
\hline Transfert & & & & & & & & - & - & घ & \\
\hline \multicolumn{12}{|c|}{ Compétences pour maintenir une bonne santé (Arcand et al., 1998; Martin \& Arcand, 2005) } \\
\hline Habileté de résolution de problèmes & $\square$ & - & & & $\mathbf{\square}$ & & $\square$ & 口 & ! & - & - \\
\hline Habileté de communication & - & च & - & " & " & च & - & I & ! & - & 口 \\
\hline Habileté à exercer son jugement critique & & & ! & 口 & - & च & " & घ & I & च & च \\
\hline Habileté à gérer son stress & & & 口 & - & & 口 & & $\mathbf{\square}$ & $\square$ & - & - \\
\hline Habileté à se fixer des buts et à les atteindre & घ & & & 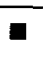 & & - & & - & - & - & - \\
\hline
\end{tabular}

Dans un premier temps, l'évolution suggérée par la séquence d'aventure Bilodeau (2005a) semble corroborer avec le développement d'habiletés pour maintenir une bonne santé (Arcand et al., 1998). Dans un deuxième temps, avant l'implantation de la séquence d'aventure, nous avons exploré à la loupe les assises du programme de formation pour le troisième cycle du primaire. 


\subsection{Les ateliers sous la loupe du PFEQ en lien avec l'éducation à la santé}

La suite d'ateliers d'éducation par l'aventure sert de point d'ancrage aux apprentissages. À titre d'exemple, les domaines d'éducation physique et à la santé, de la mathématique, de la science et de la technologie ainsi que du français, langue d'enseignement, sont majoritairement abordés à travers la séquence d'aventure. La coopération des titulaires de classe a grandement contribué à rendre les apprentissages actifs, car elles assuraient le lien entre les expériences vécues en atelier d'aventure et les apprentissages effectués en classe.

Comme le suggère Motta (1998), l'éducation à la santé doit transcender tous les programmes de formation et outrepasser le contenu disciplinaire du domaine d'éducation physique et à la santé. Ainsi, s'appuyant sur le modèle intégrateur de Godin (2002), et ce en lien avec les différents modèles et théorie de l'éducation à la santé privilégiés en Amérique du Nord (Turcotte, 2006) et Health Action Model (Tones \& Green, 2004), nous avons, avant l'expérimentation auprès des participants, effectué une classification des ateliers à l'aides des assises du domaine de formation Santé et bien-être (Tableau 3.3.1 à 3.3.3). Certes, cette classification n'est pas parfaite, mais à la lueur de notre expertise dans le domaine de l'éducation à la santé au Québec, elle nous a permis de constater que les ateliers offrent, ensemble, un portrait reflétant la majorité des facteurs clés du développement du jeune (annexe 1, tiré de Martin \& Arcand, 2005). 
De ce fait, la prochaine section de ce chapitre présente sous forme de tableau l'exercice de projection qui concerne la portée des 10 ateliers d'aventure éducative en plein air. Le tableau 3.3.1 trace un portrait de la place du domaine général de formation Santé et bien-être dans l'application de la séquence d'aventure. Le tableau 3.3.2 expose la place des deux compétences transversales d'ordre personnel et social, soit Structurer son identité et Coopérer, et leurs composantes. Tandis que le tableau 3.3.3 dégage les composantes des trois domaines d'apprentissages choisis, c'est-àdire l'éducation physique et à la santé, la science et technologie et le français.

À titre d'information, les composantes d'une compétence dynamisent la compétence et lui donnent un sens, tandis que les manifestations jouent deux rôles (Lasnier, 2000) : elles facilitent la compréhension du sens de la composante, puis permettent d'élaborer des critères pour un des aspects de l'évaluation formative. Celles-ci n'apparaissent pas dans le tableau 3.3.2, car elles sont nombreuses et variées.

La littérature rapporte que la manifestation du développement de ces compétences conduit les jeunes vers l'autonomie et l'accroissement de leur estime personnelle (Duclos, 2001; PFEQ, 2001; Martin \& Arcand, 2005). 


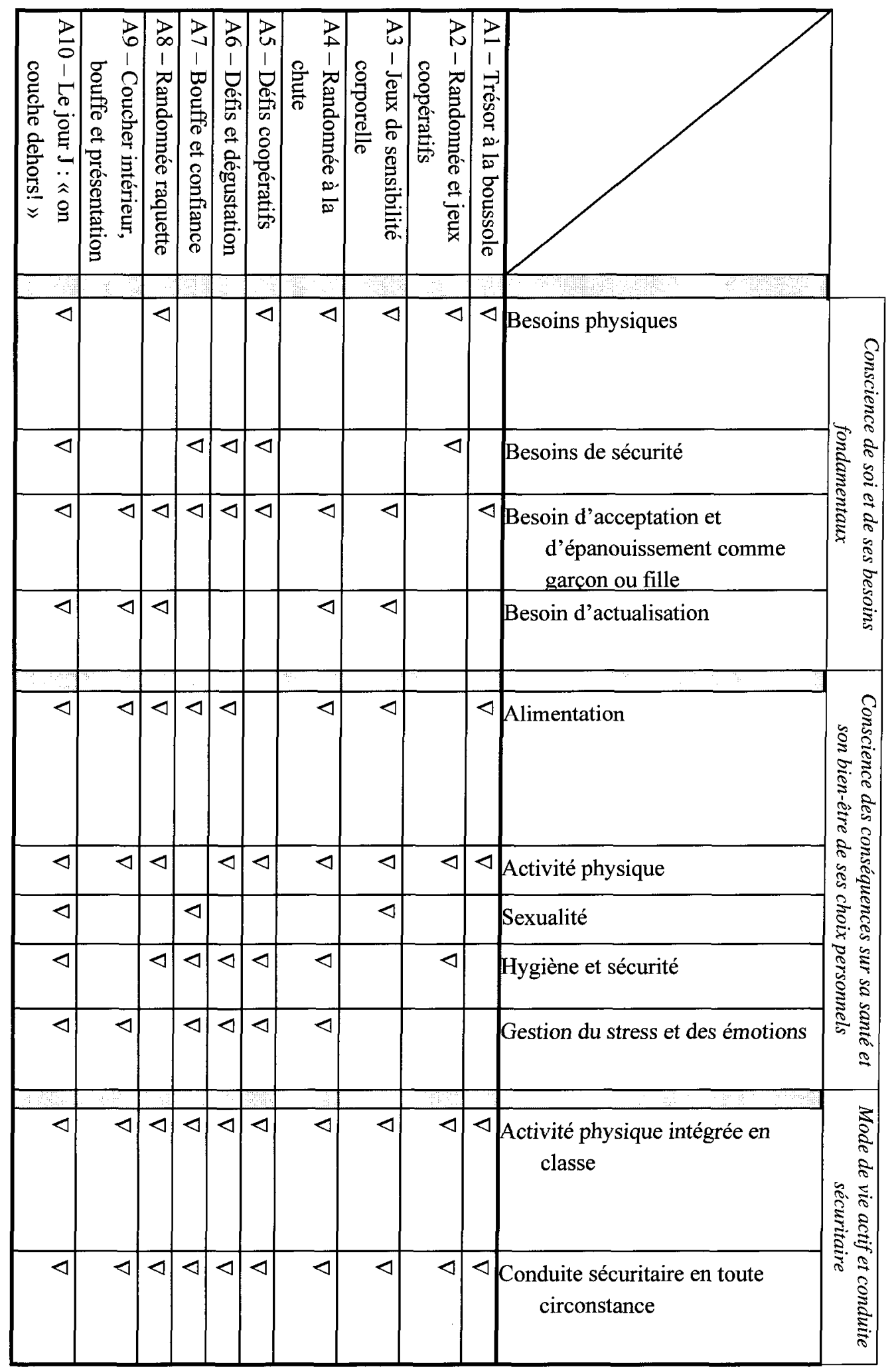

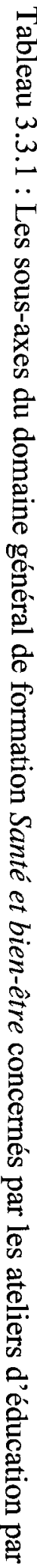


Tableau 3.3.2 : Compétences transversales d'ordre personnel et social (7 et 8 ) concernées par les ateliers d'éducation par l'aventure proposés dans le projet "Nature et Santé »

\begin{tabular}{|c|c|c|c|c|c|c|c|c|}
\hline & 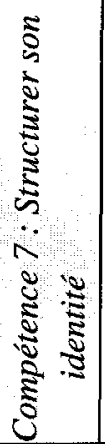 & 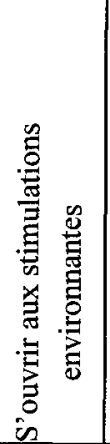 & 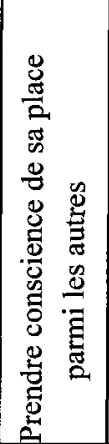 & 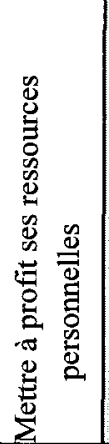 & 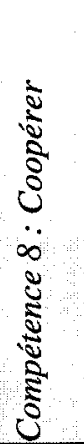 & 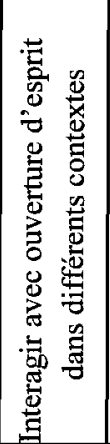 & 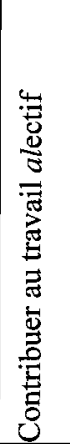 & 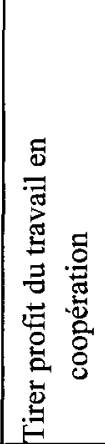 \\
\hline A1 - Trésor à la boussole & & $\nabla$ & $\nabla$ & $\nabla$ & & & $\frac{\nabla}{\nabla}$ & $\bar{\nabla}$ \\
\hline $\begin{array}{l}\text { A2 - Randonnée et jeux } \\
\text { coopératifs }\end{array}$ & & $\nabla$ & $\nabla$ & $\bar{\nabla}$ & & $\nabla$ & $\nabla$ & $\nabla$ \\
\hline $\begin{array}{l}\text { A3 - Jeux de sensibilité } \\
\text { corporelle }\end{array}$ & & $\nabla$ & $\nabla$ & $\bar{\nabla}$ & & $\nabla$ & & \\
\hline A4 - Randonnée à la chute & & $\nabla$ & $\bar{\nabla}$ & $\nabla$ & & $\nabla$ & $\bar{\nabla}$ & $\bar{\nabla}$ \\
\hline A5 - Défis coopératifs & & $\nabla$ & $\nabla$ & $\nabla$ & & $\nabla$ & $\nabla$ & $\nabla$ \\
\hline A6-Défis et dégustation & & $\bar{\nabla}$ & $\nabla$ & $\nabla$ & & $\nabla$ & $\nabla$ & $\nabla$ \\
\hline A7-Bouffe et confiance & & $\nabla$ & $\nabla$ & $\nabla$ & & $\nabla$ & $\nabla$ & $\nabla$ \\
\hline A8 - Randonnée raquette & & $\nabla$ & $\nabla$ & $\nabla$ & & $\nabla$ & $\nabla$ & $\nabla$ \\
\hline $\begin{array}{l}\text { A9- Coucher intérieur, } \\
\text { bouffe et présentation }\end{array}$ & & $\nabla$ & $\nabla$ & $\nabla$ & & $\nabla$ & $\nabla$ & $\nabla$ \\
\hline $\begin{array}{l}\text { A10 - Le jour J : « on } \\
\text { couche dehors! » }\end{array}$ & & $\nabla$ & $\nabla$ & $\nabla$ & & $\nabla$ & $\nabla$ & $\nabla$ \\
\hline Projet intégrateur & & & & & & & & \\
\hline
\end{tabular}

\subsubsection{Domaines d'apprentissage : éducation physique et à la santé, science et} technologie, français

En ce qui concerne l'acquisition de savoirs essentiels propres aux domaines d'apprentissage, ce sont les titulaires de classe qui ont piloté le projet intégrateur et effectué un suivi en classe à l'aide des stratégies d'enseignement ou d'apprentissages 
de leur choix. Le tableau 3.3.3 présente un aperçu des composantes ciblées pour le domaine des langues, de la science et de la technologie et de l'éducation physique et à la santé comme il a été planifié lors du développement des ateliers de la séquence d'aventure.

À titre d'exemple, pour la science et de la technologie, les savoirs essentiels ciblés se rapportent à différents éléments telles que L'Univers vivant, la Terre et l'espace, etc. Ainsi, l'organisation du vivant, les sources d'énergie des êtres vivants, l'étude des étoiles, de la galaxie ou du système Soleil-Terre-Lune font partie des savoirs essentiels ciblés.

Les savoirs essentiels servent à enrichir le contenu des ateliers et permettent d'assurer un suivi entre le temps vécu en classe et le temps passé en atelier. 
Tableau 3.3.3 : Domaines d'apprentissage et leurs composantes étroitement cernés par les ateliers d'éducation par l'aventure proposés dans le projet « Nature et Santé »

\begin{tabular}{|c|c|c|c|c|c|c|c|c|c|c|}
\hline & 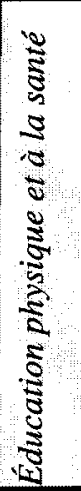 & 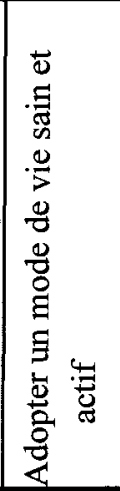 & 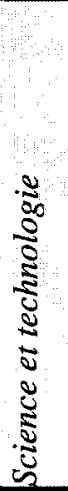 & 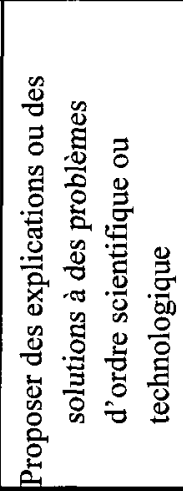 & 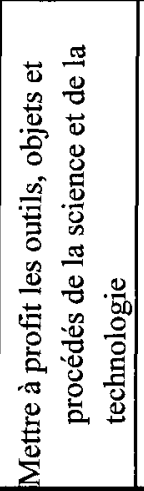 & 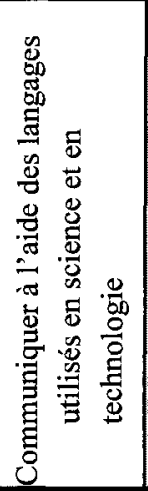 & ङ્ & 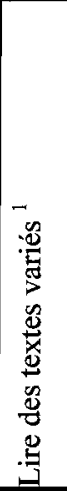 & 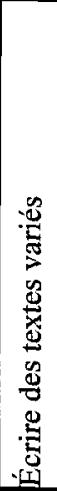 & 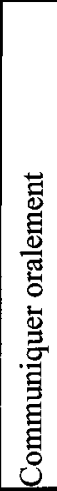 \\
\hline $\mathrm{Al}$ - Trésor à la boussole & & $\nabla$ & & $\nabla$ & $\nabla$ & & & & $\nabla$ & $\nabla$ \\
\hline $\begin{array}{l}\text { A2 - Randonnée et jeux } \\
\text { coopératifs }\end{array}$ & & $\nabla$ & & & $\nabla$ & $\nabla$ & & $\nabla$ & $\nabla$ & $\nabla$ \\
\hline $\begin{array}{l}\text { A3 - Jeux de sensibilité } \\
\text { corporelle }\end{array}$ & & $\nabla$ & & $\nabla$ & & & & & $\nabla$ & $\nabla$ \\
\hline $\begin{array}{l}\text { A4 - Randonnée à la } \\
\text { chute }\end{array}$ & & $\nabla$ & & $\nabla$ & $\nabla$ & $\nabla$ & & & $\bar{\nabla}$ & $\nabla$ \\
\hline A5 - Défis coopératifs & & $\nabla$ & & $\nabla$ & $\nabla$ & $\nabla$ & & & $\nabla$ & $\bar{\nabla}$ \\
\hline A6 - Défis et dégustation & & $\nabla$ & & & & & & & $\nabla$ & $\nabla$ \\
\hline A7 - Bouffe et confiance & & $\nabla$ & & & & & & $\nabla$ & $\nabla$ & $\nabla$ \\
\hline A8-Randonnée raquette & & $\nabla$ & & $\nabla$ & $\nabla$ & $\nabla$ & & $\nabla$ & $\nabla$ & $\nabla$ \\
\hline $\begin{array}{l}\text { A9- Coucher intérieur, } \\
\text { bouffe et présentation }\end{array}$ & & $\nabla$ & & $\bar{\nabla}$ & $\nabla$ & $\nabla$ & & & $\nabla$ & $\nabla$ \\
\hline $\begin{array}{c}\text { A10 - Le jour J : « on } \\
\text { couche dehors! » }\end{array}$ & & $\nabla$ & & $\bar{\nabla}$ & & $\bar{\nabla}$ & & & $\nabla$ & $\nabla$ \\
\hline Projet intégrateur $^{11}$ & & & & $\nabla$ & $\nabla$ & $\nabla$ & & $\nabla$ & $\nabla$ & $\nabla$ \\
\hline
\end{tabular}

\subsection{Description de la séquence d'aventure et d'apprentissage en plein air vécue} par les élèves du troisième cycle de l'école pilote

L'éducation par l'aventure et le plein air définit ce type d'apprentissage comme une approche expérientielle. La section qui suit présente la distinction entre

\footnotetext{
${ }^{11}$ La réalisation du projet final (intégrateur) a demandé une recherche sur le sujet choix et en conséquence la lecture de textes variés.
} 
les termes activités d'apprentissage, ateliers, excursion et expédition, en expliquant leur composition et la durée. L'annexe 5 présente, un exemple de fiche d'atelier avec le déroulement complet de l'atelier en lien avec la préparation nécessaire en classe.

\subsubsection{Activités d'apprentissage}

Les activités d'apprentissage comportent trois phases : préparation, réalisation et intégration (PFEQ, 2001). Dans la présente étude, la préparation et la réalisation s'effectuent successivement en classe et ensuite en atelier, tandis que la phase d'intégration se vit lors du retour sur les éléments d'apprentissage et lors de la réalisation d'une tâche intégratrice. Cette dernière phase s'effectue soit lors de l'atelier d'aventure et plein air, soit en classe et à l'aide de fiches de retour. Les activités d'apprentissage se réalisent souvent en série ce qui facilite les apprentissages globaux propres à la compétence visée (Lasnier, 2000).

\subsubsection{Ateliers d'une demi-journée classe (A1-A2-A3-A5-A6-A7-A9)}

Identifiés généralement comme des tâches intégratrices les ateliers comptent une série d'activités d'apprentissage conjointes qui visent la connaissance de soi, des autres ou de l'environnement naturel par l'exploitation d'une ou de plusieurs habiletés sociales : communication, coopération, résolution de problèmes, gestion du stress. Ces ateliers d'éducation par l'aventure ont pour objectif de favoriser le développement des compétences personnelles et sociales et de promouvoir une santé 
globale. Les expériences vécues individuellement ou en groupe mettent les intervenants scolaires et les jeunes en action en leur proposant des situations d'apprentissage actives et authentiques.

\subsubsection{Excursions et expédition $(A 4-A 8-A 10)$}

Les excursions ou l'expédition comportent un défi individuel (et de groupe) plus important que les ateliers. Elles favorisent le démarrage, l'actualisation ou l'accomplissement d'un projet. L'excursion se réalise en une journée, tandis que l'expédition implique au moins un coucher en camping ou en refuge. Les excursions et les expéditions, vécues dans un environnement sécuritaire, placent les participants en contact privilégié avec la nature. Elles sollicitent le dépassement de soi et l'affirmation de sa différence.

La prochaine section présente la composition d'une fiche d'intervention type.

\subsubsection{Fiche d'atelier}

Pour chaque atelier, excursion ou expédition, la chercheure a développé une fiche d'atelier composée de multiples éléments utiles à la cohésion des deux séquences, soit la séquence d'aventure développée par Robb et Ewert (1993, tiré de Bisson, 1999, p.207) et adaptée par Bilodeau (2005a), et la séquence d'apprentissage de Lasnier (2000) se rapportant aux assises PFEQ. En annexe 5, se trouve à titre 
d'exemple, la fiche de l'atelier A2- randonnée et jeux coopératifs qui illustre la formule adoptée. Les 10 fiches (A1 à A10) comportent une description complète des objectifs à atteindre, des thèmes abordés, des compétences transversales et disciplinaires ciblées, du matériel nécessaire à la réalisation, du déroulement, ainsi que des indications concernant la préparation et le retour en classe. Toutefois, étant donné la lourdeur du descriptif des ateliers pour l'ensemble des fiches d'intervention, celles-ci demeurent disponibles sur demande.

Chaque atelier est suivi de questions d'objectivation prenant la forme de fiches de retour (annexe 6). Ces fiches ainsi que les observations effectuées par les titulaires et la chercheure-acteure sont utilisées à titre d'outils de collecte de données.

\subsection{Les outils de collecte de données}

La présente recherche a été planifiée en utilisant plusieurs outils de collecte de données pour compenser les limites de chacun (Karsenti \& Savoie-Zajc, 2004). Ainsi, les élèves ont rempli des fiches de retour présentées sous forme de travaux scolaires orientés, les titulaires de classe ont de façon informelle participé à l'évolution de la séquence d'aventure, par leur présence lors des ateliers d'aventure et en s'exprimant librement sur l'évolution des apprentissages. Aussi, elles ont accepté de se soumettre à une entrevue semi-dirigée, tout comme l'enseignante d'éducation physique et à la santé. La direction de l'école, ainsi que les parents des 
participants ont rempli un questionnaire à leur intention et la chercheure-acteure a rigoureusement tenu un journal de bord.

\subsubsection{Fiches de retour}

Pour recueillir et stimuler la participation des enfants, chaque atelier était accompagné d'une fiche de retour qui comporte des questions d'objectivation (annexe 6). Ces fiches de retour, présentées comme des travaux scolaires orientés, devaient être complétées individuellement par chacun des élèves au meilleur de leurs connaissances et de leurs perceptions. Les 10 fiches de retour servent à dégager des informations générales et spécifiques concernant la perception des jeunes à l'égard d'eux-mêmes, d'autrui et, aussi, quant aux apprentissages vécus lors des activités.

Distribuée à la suite de la présentation du projet intégrateur à la communauté, la dernière fiche de retour explore davantage l'évolution des connaissances et le développement de la compétence Coopérer, dans la réalisation du projet intégrateur.

\subsubsection{Les entrevues}

À la suite de l'expérimentation, deux entrevues semi-dirigées ont été menées, l'une avec l'enseignante d'éducation physique et à la santé et l'autre avec les titulaires de classe. Ces entrevues visaient à mesurer plus précisément l'impact de la séquence d'aventure sur les méthodes d'enseignement des titulaires, ainsi que les 
limites d'un tel outil d'éducation à la santé pour le milieu scolaire. D'autre part, l'entrevue cherchait à évaluer l'influence des ateliers sur le développement des facteurs favorisant le maintien de la santé des jeunes participants, dont l'évolution des relations interpersonnelles au cours de l'expérimentation. Les grilles d'entrevue détaillées se trouvent aux annexes 7 et 8 .

\subsubsection{Enseignantes collaboratrices}

Au cours de la dernière semaine de classe, une entrevue d'environ une heure a été effectuée avec les deux titulaires de classe. Pour enrichir la cueillette de données, la suppléante régulière ayant participé à l'expédition finale, qui remplaçait l'enseignante numéro 1 , a été invitée à se joindre à l'entrevue semi-dirigée (annexe 7). Celle-ci a été réalisée à l'extérieur des murs de l'école, au cours de la sortie récompense de fin d'année. Libérées de leur temps d'enseignement, les enseignantes ont participé volontairement à l'exercice.

Les commentaires recueillis lors de l'entrevue semi-dirigée ont été enregistrés sur une vidéocassette et retranscrits intégralement afin de conserver la totalité des propos.

\subsubsection{Enseignante d'éducation physique et à la santé}

À la fin du projet, une entrevue de 50 minutes avec 1'enseignante du domaine de développement personnel, éducation physique et à la santé, a été réalisée. Dans le 
cadre d'une entrevue semi-dirigée, les propos ont été enregistrés sur vidéocassette et recueillis dans un endroit calme, loin du regard des élèves (annexe 8).

Tout comme pour l'entrevue réalisée avec les titulaires de classe, un compte rendu intégral a été effectué afin de conserver la totalité des propos.

\subsubsection{Questionnaires à l'intention de la direction}

En toute fin de projet, un questionnaire a été distribué à la direction de l'école afin de recueillir et de cerner son opinion sur le projet, et plus particulièrement sur sa pertinence en milieu scolaire et son impact sur le développement et la réussite des élèves. En tant que solution potentielle pour favoriser l'éducation à la santé en milieu scolaire, le questionnaire comportait aussi une partie sur les étapes d'implantation d'un tel projet (annexe 9).

\subsubsection{Questionnaires à l'intention des parents}

À la suite de l'expérimentation, un questionnaire a été distribué aux parents afin de connaître leur opinion sur leur projet, ainsi que leur perception quant à l'impact de l'expérience vécue sur le développement, le comportement et les relations interpersonnelles de leur enfant (annexe 10). Ce questionnaire n'a toutefois pas été validé auprès d'un large échantillon. 


\subsubsection{Journal de bord}

À la suite de chaque rencontre avec les jeunes et à la suite des rencontres d'autorégulation avec les enseignantes, la chercheure-acteure a transcrit systématiquement des réflexions dans son journal de bord. Elle y a noté ses impressions et relaté les événements (Karsenti \& Savoie-Zajc, 2004). À cette étape, un constat sur la réalité peut être formulé sous forme de réflexion personnelle. Cela permet d'évaluer sommairement la situation, d'entrevoir l'évolution des actions éducatives (Savoie-Zajc, 2001) et de cibler les adaptations qui s'imposent. L'ajustement des actions éducatives contribue à perfectionner la pratique dans le développement des compétences personnelles et sociales, ainsi que l'adoption de saines habitudes de vie. La rédaction du journal de bord sert aussi à relever les faits saillants des ateliers, ainsi que les attitudes et comportements des jeunes au cours de l'activité.

Finalement, les nombreuses lectures et relectures du journal de bord ont servi à identifier les éléments constitutifs de la dynamique de terrain afin de reconstituer, une fois le travail terrain terminé, l'atmosphère qui a prévalu au cours de la recherche (Karsenti \& Savoie-Zajc, 2004; Paillé \& Mucchielli, 2008). 


\subsection{Déontologie}

\subsubsection{Lettre de consentement destinée aux parents}

Les parents ont été formellement rencontrés en début de projet et tous ont signé une lettre de consentement (annexe 4). Lors de l'atelier 9, quelques semaines avant 1'expédition, une seconde rencontre de mise au point a été organisée. L'objectif de cette rencontre était de rassurer principalement les parents sur le déroulement de la sortie finale (l'expédition) au Lac Emmuraillé. Lors de cette rencontre, les deux titulaires de classe, l'intervenante (chercheure-actrice) et les deux accompagnateurs spécialisés en plein air et tourisme d'aventure (annexe 12) étaient présents.

\subsubsection{Plan d'urgence}

Le plan d'urgence a été adapté, à la réalité du milieu, par un étudiant au baccalauréat en plein air et tourisme d'aventure, de l'Université du Québec à Chicoutimi, afin d'assurer la sécurité des participants et de rassurer les parents. 


\subsection{Analyse du contenu}

L'analyse de contenu cherche à savoir ce qui se cache derrière les paroles et les actions (Bardin, 2007). Ainsi, au cours de la réalisation de la séquence d'aventure et d'apprentissage, un ensemble d'outils de collecte de données (voir section 3.5, du présent chapitre) et de technique d'analyse (triangulation, catégorisation, analyse phénoménologique et de l'analyse thématique) ont été utilisés afin d'obtenir une production explicite de connaissances conduisant à des résultats fiables (Bardin, 2007).

L'analyse de contenu du présent mémoire est d'abord réalisée par la triangulation et catégorisation des propos émis par les différents acteurs impliqués afin d'en faire émerger les multiples facteurs clés du développement des jeunes (annexe 1).

\subsubsection{Triangulation et catégorisation}

Selon Karsenti et Savoie-Zajc (2004, p. 146) la triangulation poursuit deux objectifs : «Le premier est de permettre au chercheur d'explorer le plus de facettes possible du même problème étudié en recueillant des données qui font ressortir des perceptives diverses. Le second vise à mettre la triangulation au cœur du processus de co-construction des connaissances et à supporter l'objectivation du sens produit 
pendant la recherche. » En ce qui concerne le présent mémoire, les instruments utilisés sont :

a) Les écrits recueillis dans les fiches de retour remplies par les jeunes participants après chaque atelier.

b) Les commentaires et les propos des titulaires de classe, de l'enseignante d'éducation physique et à la santé, des parents et de la direction d'école.

c) Les perceptives recueillies dans le récit du journal de bord de la chercheure-acteure.

Par la suite, la ventilation des résultats obtenus a été effectuée à l'aide d'une opération de classification d'éléments constructifs recueillis par les outils de collecte de données qui ont servi d'assise à la catégorisation (Bardin, 2007). Comme il est expliqué précédemment, la ventilation des citations, propos ou écrits des participants, des intervenants scolaires et de la chercheure-acteure, a été effectuée en s'appuyant sur les assises du PFEQ, par catégories identifiées.

La catégorisation est une opération de classification d'éléments constitutifs d'un ensemble par différenciation, puis par regroupement analogique des critères préalablement définis (Bardin, 2007). En ce qui concerne le présent mémoire, il s'agit de démontrer l'impact de la séquence d'aventure comme outil d'éducation à la santé en milieu scolaire. Les résultats recueillis à l'aide des différents outils de collecte de données ont été agencés principalement en fonction des axes et sous-axes 
du DGF Santé et bien-être (tableau 3.3.4) et des six composantes des compétences transversales d'ordre personnel et social (tableaux 3.3.5 et 3.3.6). Les différentes descriptions des compétences transversales et du DGF Santé et bien-être fournies dans le PFEQ (PFEQ, 2001) sont à la fois complémentaires et pertinentes pour le milieu scolaire et souscrivent aux principes de l'éducation à la santé. Aussi, elles illustrent clairement les variables traitées dans l'analyse des résultats et favorisent également leur homogénéisation (Bardin, 2007). 
Tableau 3.3.4 : Exemple de relevé des thèmes pour le DGF Santé et bien-être

\begin{tabular}{|c|c|c|c|}
\hline $\begin{array}{l}\text { Domaine général de } \\
\text { formation (rubrique) }\end{array}$ & Axes (Sous-rubrique) & Sous-axes (thèmes) & $\begin{array}{l}\text { Extraits des outils de } \\
\text { collecte de données }\end{array}$ \\
\hline \multirow[t]{3}{*}{$\begin{array}{l}\text { DGF } \\
\text { Santé et bien-être }\end{array}$} & $\begin{array}{l}\text { Conscience de soi et de } \\
\text { ses besoins } \\
\text { fondamentaux }\end{array}$ & $\begin{array}{l}\text { Besoins physiques } \\
\text { Besoins de sécurité } \\
\text { Besoin d'acceptation et } \\
\text { d'épanouissement comme } \\
\text { garçon ou fille }\end{array}$ & $\begin{array}{l}\text { "extraits des écrits } \\
\text { recueillis dans les fiches } \\
\text { de retour remplies par les } \\
\text { élèves» } \\
\text { " commentaires et propos } \\
\text { des titulaires de classe, de } \\
\text { l'enseignante d'éducation } \\
\text { physique et à la santé, } \\
\text { entrevues » } \\
\text { "réponses de la direction } \\
\text { et des parents aux } \\
\text { questionnaires » } \\
\text { "extraits du journal de } \\
\text { bord de la chercheure- } \\
\text { actrice» }\end{array}$ \\
\hline & $\begin{array}{l}\text { Conscience des } \\
\text { conséquences sur sa } \\
\text { santé et son bien-être } \\
\text { de ses choix } \\
\text { personnels }\end{array}$ & $\begin{array}{l}\text { Alimentation } \\
\text { Activité physique } \\
\text { Sexualité } \\
\text { Hygiène et sécurité }\end{array}$ & $\begin{array}{l}\text { "extraits des écrits } \\
\text { recueillis dans les fiches } \\
\text { de retour remplies par les } \\
\text { élèves » } \\
\text { " Commentaires et propos } \\
\text { des titulaires de classe, de } \\
\text { l'enseignante d'éducation } \\
\text { physique et à la santé, } \\
\text { entrevues » } \\
\text { "réponses de la direction } \\
\text { et des parents aux } \\
\text { questionnaires" } \\
\text { «extraits du journal de } \\
\text { bord de la chercheure- } \\
\text { acteure» }\end{array}$ \\
\hline & $\begin{array}{l}\text { Mode de vie actif et } \\
\text { conduite sécuritaire }\end{array}$ & $\begin{array}{l}\text { Activité physique } \\
\text { intégrées en classe } \\
\text { Conduite sécuritaire en } \\
\text { toute circonstance }\end{array}$ & Etc. \\
\hline
\end{tabular}


Tableau 3.3.5: Exemple de relevé des thèmes pour la compétence transversale Structurer son identité

\begin{tabular}{|c|c|c|c|}
\hline $\begin{array}{l}\text { Compétences } \\
\text { (rubriques) }\end{array}$ & $\begin{array}{l}\text { Composantes } \\
\text { (Sous-rubriques) }\end{array}$ & Manifestations (thèmes) & $\begin{array}{l}\text { Extraits des outils de collecte de } \\
\text { données }\end{array}$ \\
\hline \multirow[t]{3}{*}{$\begin{array}{l}\text { Structurer son } \\
\text { identité }\end{array}$} & $\begin{array}{l}\text { S'ouvrir aux } \\
\text { stimulations } \\
\text { environnantes }\end{array}$ & $\begin{array}{l}\text { Réagir aux faits, aux } \\
\text { situations ou aux événements. } \\
\text { Identifier ses perceptions, ses } \\
\text { sentiments, ses réflexions à } \\
\text { leur égard. Percevoir } \\
\text { l'influence du regard des } \\
\text { autres sur ses réactions. } \\
\text { Augmenter son bagage } \\
\text { culturel par les échanges, la } \\
\text { lecture et le contact avec des } \\
\text { œuvres médiatiques variées. } \\
\text { Accueillir les références } \\
\text { morales et spirituelles } \\
\text { de son milieu. }\end{array}$ & $\begin{array}{l}\text { " extraits des écrits recueillis } \\
\text { dans les fiches de retour } \\
\text { remplies par les élèves " } \\
\text { "commentaires et propos des } \\
\text { titulaires de classe, de } \\
\text { l'enseignante d'éducation } \\
\text { physique et à la santé, } \\
\text { entrevues» } \\
\text { "réponses de la direction et des } \\
\text { parents aux questionnaires» } \\
\text { "extraits du journal de bord de } \\
\text { la chercheure-acteure» }\end{array}$ \\
\hline & $\begin{array}{l}\text { Prendre } \\
\text { conscience de sa } \\
\text { place parmi les } \\
\text { autres }\end{array}$ & $\begin{array}{l}\text { Reconnaître ses valeurs et } \\
\text { ses buts. Se faire confiance. } \\
\text { Développer ses opinions et } \\
\text { ses choix. } \\
\text { Reconnaître son appartenance } \\
\text { à une collectivité. } \\
\text { Manifester une ouverture à la } \\
\text { diversité culturelle et } \\
\text { ethnique. }\end{array}$ & $\begin{array}{l}\text { "extraits des écrits recueillis } \\
\text { dans les fiches de retour } \\
\text { remplies par les élèves" } \\
\text { "commentaires et propos des } \\
\text { titulaires de classe, de } \\
\text { l'enseignante d'éducation } \\
\text { physique et à la santé, entrevues } \\
\text { " } \\
\text { "réponses de la direction et des } \\
\text { parents aux questionnaires » } \\
\text { "extraits du journal de bord de } \\
\text { la chercheure-acteure» }\end{array}$ \\
\hline & $\begin{array}{l}\text { Mettre à profit ses } \\
\text { ressources } \\
\text { personnelles }\end{array}$ & $\begin{array}{l}\text { Exploiter ses forces et } \\
\text { surmonter ses limites. } \\
\text { Juger de la qualité et de la } \\
\text { pertinence de ses choix } \\
\text { d'action. } \\
\text { Manifester de plus en plus } \\
\text { d'autonomie et } \\
\text { d'indépendance. }\end{array}$ & Etc. \\
\hline
\end{tabular}


Tableau 3.3.6 : Exemple de relevé des thèmes la compétence transversale Coopérer

\begin{tabular}{|c|c|c|c|}
\hline $\begin{array}{l}\text { Compétences } \\
\text { (rubriques) }\end{array}$ & $\begin{array}{l}\text { Composantes } \\
\text { (Sous-rubriques) }\end{array}$ & Manifestations (thèmes) & $\begin{array}{l}\text { Extraits des outils de collecte de } \\
\text { données }\end{array}$ \\
\hline \multirow{3}{*}{ Coopérer } & $\begin{array}{l}\text { Interagir avec } \\
\text { ouverture d'esprit } \\
\text { dans différents } \\
\text { contextes }\end{array}$ & $\begin{array}{l}\text { Accueillir l'autre avec ses } \\
\text { caractéristiques. } \\
\text { Être attentif à l'autre et } \\
\text { reconnaître ses intérêts et } \\
\text { ses besoins. } \\
\text { Échanger des points de vue } \\
\text { avec l'autre, écouter et } \\
\text { accueillir } \\
\text { les divergences. } \\
\text { Adapter son comportement. }\end{array}$ & $\begin{array}{l}\text { "extraits des écrits recueillis dans } \\
\text { les fiches de retour remplies par } \\
\text { les élèves" } \\
\text { " commentaires et propos des } \\
\text { titulaires de classe, de } \\
\text { l'enseignante d'éducation } \\
\text { physique et à la santé, entrevues" } \\
\text { " réponses de la direction et des } \\
\text { parents aux questionnaires» } \\
\text { " extraits du journal de bord de la } \\
\text { chercheure-acteure» }\end{array}$ \\
\hline & $\begin{array}{l}\text { Contribuer au } \\
\text { travail collectif }\end{array}$ & $\begin{array}{l}\text { Participer aux activités de la } \\
\text { classe et de l'école de façon } \\
\text { active et dans un esprit de } \\
\text { collaboration. } \\
\text { Planifier et } \\
\text { réaliser un travail avec } \\
\text { d'autres. } \\
\text { Accomplir sa } \\
\text { tâche selon les règles } \\
\text { établies en groupe. }\end{array}$ & $\begin{array}{l}\text { "extraits des écrits recueillis dans } \\
\text { les fiches de retour remplies par } \\
\text { les élèves » } \\
\text { " commentaires et propos des } \\
\text { titulaires de classe, de } \\
\text { l'enseignante d'éducation } \\
\text { physique et à la santé, entrevues » } \\
\text { « réponses de la direction et des } \\
\text { parents aux questionnaires" } \\
\text { "extraits du journal de bord de la } \\
\text { chercheure-acteure» }\end{array}$ \\
\hline & $\begin{array}{l}\text { Tirer profit du } \\
\text { travail en } \\
\text { coopération }\end{array}$ & $\begin{array}{l}\text { Reconnaitre les tâches qui } \\
\text { seront réalisées plus } \\
\text { efficacement dans un travail } \\
\text { collectif. } \\
\text { Apprécier sa participation et } \\
\text { celle de ses pairs à un } \\
\text { travail d'équipe. } \\
\text { Identifier les éléments qui } \\
\text { ont facilité ou entravé la } \\
\text { coopération. } \\
\text { Cerner les améliorations } \\
\text { souhaitables pour sa } \\
\text { prochaine participation à un } \\
\text { travail collectif. }\end{array}$ & Etc. \\
\hline
\end{tabular}




\subsubsection{Examen phénoménologique et analyse thématique}

L'examen de la cueillette de données issue de l'introduction d'un phénomène nouveau en milieu scolaire, soit la réalisation d'une séquence d'aventure et d'apprentissage, selon les auteurs consultés (Paillé \& Mucchielli, 2008), est de type phénoménologique. D'autre part, la catégorisation des données a été effectuée en tenant compte d'arbres thématiques articulés selon les assises du PFEQ et selon les principes de l'analyse thématique (Paillé \& Mucchielli, 2008; Bardin, 2007).

L'examen phénoménologique est une construction sociale qui appartient aux acteurs de la situation (Paillé \& Mucchielli, 2008). Dans le cas de la présente recherche, les élèves, les titulaires de classe et l'intervenante (chercheure-acteure) se sont tous retrouvés au cour de l'action. L'expérimentation produite propose une nouvelle démarche pour le milieu de l'éducation et de la santé. Les multiples outils de collecte de données utilisés ont permis, une fois agencés et classifiés, la compréhension du phénomène étudié. Ensemble, ils diffusent la réalité humaine dans laquelle l'expérimentation et les apprentissages ont été réalisés. La séquence d'aventure et d'apprentissage a créé des situations de défis personnels et de groupe, notamment sur le plan de la relation avec l'autorité et avec les pairs. L'expérimentation a donné lieu à l'émergence d'une multitude d'émotions générées par les conditions physiques et environnementales et par les apprentissages proposés dans l'action. Pour Paillé et Mucchielli (2008), l'examen phénoménologique émerge lorsque le chercheur prend acte, suit dans le détail et trace un portrait de la situation 
vécue par les acteurs. Dans le cas de la séquence d'aventure et d'apprentissage, les élèves et l'ensemble des intervenants scolaires sont tous des acteurs de la situation.

La force de cette analyse réside dans l'importance accordée au potentiel de la réalisation de l'expérimentation de la séquence proposée sur le terrain (Paillé \& Mucchielli, 2008). Le temps accordé à l'analyse des différents événements émergents de l'expérimentation a produit un message authentique et sans équivoque chez la chercheure-acteure.

Comme il a été mentionné antérieurement, l'analyse des résultats de la réalisation de la séquence d'éducation par l'aventure en milieu scolaire repose sur le domaine général de formation (DGF) Santé et bien-être, ainsi que sur les deux compétences transversales d'ordre personnel et social. Le choix de ces thèmes d'analyse permet de tracer un portrait des apprentissages susceptible de ressortir pendant et à la suite de la réalisation de l'expérimentation. Ces thèmes ont permis la conception de grilles d'analyse sous la forme d'arbres thématiques. Par conséquent, pour la présente étude, les appellations «axe » et «sous-axe » sont utilisées pour illustrer les éléments relatifs au DGF Santé et bien-être, tandis que les termes « composantes » et «manifestations » sont utilisés au lieu des appellations sousrubriques et thèmes, comme ils ont été proposés par Paillé et Mucchielli (2008). Les tableaux 3.3.4 à 3.3.6 présentés précédemment illustrent la logique de la classification effectuée. 
L'analyse thématique consiste en un regroupement des thèmes abordés dans les comptes rendus intégraux, les questionnaires, les fiches de retour orientées et les notes d'observation recueillies dans le journal de bord. L'analyse du contenu subjectif vise à démontrer que les impressions et les jugements intuitifs véhiculés au cours de la réalisation des ateliers conduisent à des résultats fiables (Bardin, 2007).

Le but est d'identifier les points communs des expériences et des événements vécus par les élèves et les intervenants scolaires (titulaires et chercheure-acteure) et de présenter, à l'aide de ces grilles thématiques, le contenu des apprentissages véhiculés et les compétences développées, lors de la réalisation de la séquence d'aventure et d'apprentissage. Les grilles thématiques (tableaux 3.3.4 à 3.3.6) présentent les rubriques (DGF ou compétences), sous-rubriques (axes ou composantes) et thèmes (sous-axes ou manifestations) qui servent d'indicateurs pour produire les connaissances par catégorisation et fournir par condensation une représentation simplifiée des données brutes (Bardin, 2007). Cette technique d'analyse conduit à une déduction logique (ou inférence) des connaissances relatives à la réception des différents messages émergents au cours de réalisation de la séquence d'aventure et d'apprentissage. Ainsi la décomposition-reconstruction, joue un rôle dans l'identification de correspondance entre les messages et la réalité sousjacente (Bardin, 2007). 
L'exercice est forcément interprétatif; or, les arbres thématiques utilisés, basés sur le PFEQ (PFEQ, 2001), sont bien articulés et suffisamment éloquents pour assurer une vraisemblance aux données émergentes.

Afin de standardiser le vocable utilisé pour l'analyse des résultats, les documents émis par le MELS concernant l'approche École en santé (Martin \& Arcand, 2005), et plus précisément les facteurs clés du développement des jeunes sous l'angle individuel (annexe 1), ont servi d'assise.

Finalement, dans la dernière partie de l'analyse, à titre informel, un regard a été porté sur la pluridisciplinarité, ainsi que sur la pertinence de son implantation en milieu scolaire. 


\section{CHAPITRE 4}

\section{Résultats et analyse}

Cette recherche a pour but d'évaluer les effets de l'intégration d'ateliers éducatifs réalisés dans un contexte d'aventure en plein air sur le développement d'une conscientisation en matière de santé auprès d'un groupe d'élèves du troisième cycle du primaire. Dans le but d'arrimer l'analyse des résultats aux visées du MELS et du MSSS, le vocable établi par l'approche École en santé (AES) (Martin \& Arcand, 2005) sera utilisé.

La première partie de l'analyse présente les visées éducatives en matière de Santé et bien-être de chaque atelier. La deuxième partie aborde le développement de l'estime de soi, des compétences sociales selon les manifestations observées pour chacune des composantes des deux compétences transversales de l'ordre personnel et social. La troisième partie traite du volet des habitudes de vie, en lien avec l'acquisition de connaissances ciblées par la troisième compétence du domaine personnel : Éducation physique et à la santé. Cette partie présente également des éléments ayant trait à la conduite sécuritaire dans les loisirs et les sports. La quatrième partie expose la synthèse des résultats de la séquence d'aventure. 
Finalement, la cinquième partie présente l'opinion des enseignants envers le potentiel de la séquence d'aventure et d'apprentissage sur la pluridisciplinarité.

\subsection{Les visées éducatives en matière de santé et de bien-être et les cibles atteintes pour chaque atelier}

Au cours de la présente étude, 29 jeunes du troisième cycle du primaire du de l'école pilote ont vécu, à l'intérieur du cadre scolaire, 10 ateliers d'éducation par l'aventure en plein air qui comprenaient plusieurs éléments constitutifs du PFEQ. La conception de la séquence d'aventure et d'apprentissage s'est appuyée sur le procédé mis de l'avant par Robb et Ewert (1993, tiré de Bisson, 1999, p. 207) visant le développement des habiletés suggérées par Arcand et al. (1998) pour le maintien d'une bonne santé (voir tableau 3.2.1).

Les ateliers comportent des défis qui exigent l'utilisation des habiletés de communication, de résolution de problèmes et le développement d'une confiance (respect) mutuelle pour atteindre les buts suggérés. Il est toutefois essentiel de mentionner que les visées éducatives en matière de santé et de bien-être créent un ancrage pour la progression de la séquence des ateliers. Les tableaux 4.1.1 à 4.1.3 font état des intentions éducatives reliées à la réalisation de chacune des activités.

Afin d'identifier les retombées potentielles d'un tel mode d'intervention sur la conscientisation en matière de santé chez les participants, un portrait de l'évolution 
de la perception des jeunes a été tracé en rassemblant, par atelier, leurs réponses écrites et orales.

\subsubsection{L'émergence de la connaissance en matière de Santé et bien-être auprès des élèves}

De façon générale, les observations effectuées par les titulaires de classe et la chercheure viennent appuyer les propos des jeunes. À mi-parcours, à la suite de la réalisation de 1'atelier 6 , les commentaires des jeunes et des titulaires de classe dénotent une progression des connaissances en matière de santé et de bien-être, par exemple, se positionner adéquatement pour accroître son endurance ou inclure, à titre de collation, davantage de fruits (tableau 4.5.1, p. 161).

Dans le même ordre d'idées, les troisièmes colonnes des tableaux 4.1.1 à 4.1.3 intitulées : «cibles atteintes », présentent l'état de la situation comme elle est perçue par la chercheure-acteure, à la suite de la réalisation de chaque atelier. Finalement, le contenu des encadrés, observations de la chercheure, relate brièvement quelques faits saillants de l'atelier en question, par l'observation de réactions d'élèves ou par les échanges verbaux entretenus avec les titulaires de classe. Bien que tous les ateliers poursuivent des objectifs précis en matière d'éducation à la santé, c'est l'ensemble de la séquence vécue qui modifie les intentions et transforme les comportements en actions de santé (Tones \& Green, 2004; Godin, 2002). 
D'ailleurs, il est intéressant d'observer que le nombre de commentaires pertinents s'accroît au cours de la réalisation de la séquence d'aventure et d'apprentissage. La chercheure-acteure attribue l'évolution du contenu des échanges verbaux sur la santé et le bien-être aux changements de perception des élèves et à l'amélioration progressive de la zone de confort des participants sur les plans individuel, environnemental et social (Bilodeau, 2001; 2005b). 
Tableau 4.1.1 : Intentions éducatives et cibles atteintes en matière de Santé et bien-être pour les ateliers 1 à 5

\begin{tabular}{|c|l|l|}
\hline & $\begin{array}{c}\text { Intention éducative en matière de } \\
\text { Santé et bien-être }\end{array}$ & $\begin{array}{l}\text { Cibles atteintes } \\
\text { (ou partiellement atteintes) }\end{array}$ \\
\hline $\begin{array}{c}\text { A1 - Trésor à la } \\
\text { boussole }\end{array}$ & Adaptation à l'environnement & $\begin{array}{c}\text { Éveil sensoricorporel } \text { envers } \\
\text { soi-même et à }\end{array}$ \\
& physique. & l'environnement. \\
& Conscience de soi. & Habileté à communiquer. \\
\hline
\end{tabular}

Observations de la chercheure

Les jeunes sont animés et participent bien. Je sens un désir de partage et d'ouverture envers la nouveauté. (voir annexe 11)

\begin{tabular}{|l|l|l|}
\hline $\begin{array}{c}\text { A2 - Randonnée et jeux } \\
\text { coopératifs }\end{array}$ & $\begin{array}{c}\text { Habileté à communiquer et à résoudre } \\
\text { des problèmes. } \\
\text { Habiletés psychomotrices. }\end{array}$ & $\begin{array}{l}\text { Habileté à communiquer et à } \\
\text { coopérer. } \\
\text { Coordination corporelle. }\end{array}$ \\
\hline
\end{tabular}

Observations de la chercheure

Ateliers intéressants pour observer l'élaboration de stratégies de communication au sein du groupe classe. Les titulaires de classe ont remarqué la présence de leaders différents. Offrir plus de temps à l'activité sur l'étude de l'écosystème.

\begin{tabular}{|l|l|l|}
\hline $\begin{array}{c}\text { A3 - Jeux de sensibilité } \\
\text { corporelle }\end{array}$ & $\begin{array}{l}\text { Habiletés de coordination. } \\
\text { Adaptation à l'environnement } \\
\text { physique. }\end{array}$ & $\begin{array}{l}\text { Régulation personnelle. } \\
\text { Connaissances. }\end{array}$ \\
\hline
\end{tabular}

Observations de la chercheure

Atelier qui semble avoir été très apprécié, car les jeunes ont pris soin de me le mentionner et de me remercier personnellement. Lors de l'exercice d'imagerie mentale jumelée à des jeux d'habiletés physiques, $\mathrm{j}$ 'ai perçu le maintien de concentration et la manifestation de détente mentale. Connaissance explorée : le phénomène de la respiration.

\begin{tabular}{|l|l|l|}
\hline $\begin{array}{c}\text { A4 - Randonnée à la } \\
\text { chute }\end{array}$ & $\begin{array}{l}\text { Adaptation à l'environnement } \\
\text { physique. } \\
\text { Interactions sociales. }\end{array}$ & $\begin{array}{l}\text { Connaissances sur : } \\
\text { l'alimentation; }\end{array}$ \\
& $\begin{array}{l}\text { Connaissances : } \\
\text { l'activité physique (bienfait } \\
\text { physiologique); }\end{array}$ \\
& $\begin{array}{l}\text { l'alimentation; } \\
\text { l'activité physique (Bienfait } \\
\text { physiologique); }\end{array}$ & $\begin{array}{l}\text { Habiletés : } \\
\text { psychomotrices; } \\
\text { communication; }\end{array}$ \\
& l'environnement naturel. \\
& Développer la condition physique. & se fixer des buts et les atteindre. \\
& & Satisfaction personnelle. \\
\hline
\end{tabular}

Observations de la chercheure

Tous les élèves ont réussi, quel que soit leur potentiel initial. J'ai profité des temps de pause et de ravitaillement pour enrichir les connaissances en matière d'alimentation et de pratique d'activité physique responsable (comportements sains et sécuritaires). J'ai perçu une grande réception de la part des élèves.

\begin{tabular}{|l|l|l|}
\hline A5 - Défis coopératifs & $\begin{array}{l}\text { Habileté à résoudre des problèmes, } \\
\text { communiquer et coopérer. } \\
\text { Adaptation à l'environnement } \\
\text { physique. }\end{array}$ & $\begin{array}{l}\text { Connaissance de soi. } \\
\text { Habileté à coopérer. } \\
\text { Adaptation à l'environnement } \\
\text { physique et socioculturel. }\end{array}$ \\
\hline
\end{tabular}

Observations de la chercheure

Les conditions climatiques ( vent et froid) et le manque de formation de l'une des titulaires de classe pour l'utilisation du matériel (boussole) ont compliqué la réalisation de l'atelier. Tout le monde (jeunes et intervenants scolaires) a effectué des apprentissages sur les plans personnel et social. L'enseignante 2 estime que malgré le froid, cet atelier a été constructif et formateur pour les jeunes. Connaissance acquise : tenue vestimentaire adéquate. 
Tableau 4.1.2 : Intentions éducatives et cibles atteintes en matière de Santé et bien-être pour les ateliers 6 à 8

\begin{tabular}{|l|l|l|}
\hline & $\begin{array}{l}\text { Intention éducative en matière de } \\
\text { Santé et bien-être }\end{array}$ & $\begin{array}{l}\text { Cibles atteintes } \\
\text { (ou partiellement atteintes) }\end{array}$ \\
\hline $\begin{array}{c}\text { A6 - Défis et } \\
\text { dégustation }\end{array}$ & $\begin{array}{l}\text { Conscience de soi; habileté } \\
\text { psychomotrices } \\
\text { Habileté à } \\
\text { gérer son stress; } \\
\text { communiquer; } \\
\text { exercer son jugement critique. }\end{array}$ & $\begin{array}{l}\text { Régulation personnelle dans la } \\
\text { gestion du stress. } \\
\text { Habileté à } \\
\text { résoudre des problèmes; } \\
\text { exercer son jugement critique; } \\
\text { coopérer. }\end{array}$ \\
\hline
\end{tabular}

Observations de la chercheure

Plus habiles dans leurs mouvements, les jeunes travaillent ensemble. Ils sont attentifs, se déplacent en se taquinant et en chantant. Les activités proposées dans l'atelier 6 demandent de la concentration ainsi que du respect mutuel. Bien que la performance individuelle soit importante, c'est le résultat de l'équipe qui prime. Les titulaires de classe disent aimer voir évoluer les jeunes dans un autre contexte que la classe. Elles disent découvrir la VRAIE personnalité des jeunes. Connaissance en évolution : l'utilisation du langage approprié et la connaissance de soi.

\begin{tabular}{|l|l|l|}
\hline A7 - Bouffe et confiance & $\begin{array}{l}\text { Connaissance sur l'alimentation. } \\
\text { Confiance mutuelle. }\end{array}$ & $\begin{array}{l}\text { Connaissance sur l'alimentation } \\
\text { Confiance mutuelle : habileté à } \\
\text { avoir des relations sociales } \\
\text { de qualité. }\end{array}$ \\
\hline
\end{tabular}

Observations de la chercheure

À partir de maintenant, nous sommes en phase de préparation pour réaliser l'expédition. Les jeunes fonctionnent bien et les titulaires de classe sont prêtes à poursuivre. Cet atelier demande du temps passé en classe. Bien que les jeunes participent, je sens un léger manque d'intérêt. Le contenu de l'atelier est trop important, c'est-à-dire que les activités proposées sont trop nombreuses et trop difficiles pour le groupe.

\begin{tabular}{|c|c|c|}
\hline $\begin{array}{l}\text { A8 - Randonnée } \\
\text { raquette }\end{array}$ & $\begin{array}{l}\text { Habiletés: } \\
\text { Psychomotrices; } \\
\text { à se fixer des buts et les atteindre; } \\
\text { à gérer son stress; } \\
\text { Connaissances : } \\
\text { l'activité physique (bienfait } \\
\quad \text { physiologique et psychologique). } \\
\text { L'environnement naturel. }\end{array}$ & $\begin{array}{l}\text { Eveil sensoricorporel envers soi- } \\
\quad \text { même et l'environnement } \\
\text { Connaissances sur : } \\
\text { alimentation; } \\
\text { l'activité physique (bienfaits } \\
\quad \text { physiologiques et } \\
\quad \text { psychologiques); } \\
\text { l'environnement naturel; } \\
\text { Habiletés } \\
\text { Psychomotrices : } \\
\text { interactions sociales; } \\
\text { se fixer des buts et les atteindre; } \\
\text { Comportement sain et sécuritaire. } \\
\text { Satisfaction personnelle. }\end{array}$ \\
\hline \multicolumn{3}{|c|}{$\begin{array}{l}\text { Observations de la chercheure } \\
\text { Premier essai raquette, les jeunes sont animés. Cet atelier semble attendu et propose un amalgame } \\
\text { d'activités de coopération et d'acclimatation servant à consolider les connaissances acquises et } \\
\text { certaines habiletés psychomotrices. Tout au long du parcours, les jeunes partagent leur découverte, } \\
\text { s'entraident, se taquinent. Ils semblent avoir apprécié leur journée, ils m'ont dit être fiers d'eux et } \\
\text { avoir adoré les activités collectives. }\end{array}$} \\
\hline
\end{tabular}


Tableau 4.1.3 : Intentions éducatives et cibles-atteintes en matière de-Santé et bien-être pour les ateliers 9 et 10

\begin{tabular}{|l|l|l|}
\hline & $\begin{array}{l}\text { Intention éducative en matière de } \\
\text { Santé et bien-être }\end{array}$ & $\begin{array}{l}\text { Cibles atteintes } \\
\text { (ou partiellement atteintes) }\end{array}$ \\
\hline $\begin{array}{l}\text { A9 - Coucher intérieur, } \\
\text { bouffe et présentation }\end{array}$ & $\begin{array}{l}\text { Habileté à : } \\
\text { communiquer; } \\
\text { coopérer; } \\
\text { résoudre des problèmes; } \\
\text { exercer son jugement critique. } \\
\text { Connaissances sur : } \\
\text { l'alimentation. } \\
\text { Comportements sains et sécuritaires. }\end{array}$ & $\begin{array}{l}\text { Connaissances sur : } \\
\text { l'alimentation; } \\
\text { Habiletés : } \\
\text { résoudre des problèmes; } \\
\text { communiquer; } \\
\text { coopérer; } \\
\text { Satisfaction personnelle. }\end{array}$ \\
\hline $\begin{array}{l}\text { Observations de la chercheure } \\
\text { Les jeunes sont très excités, ils sont incapables d'accomplir la chaise humaine, un exercice qu'ils ont } \\
\text { effectué avec brio plus d'une fois. Cet exercice demande d'exploiter leur habileté de coopération et } \\
\text { de gestion du stress. Il est toutefois intéressant de constater que quelques élèves parmi le groupe sont } \\
\text { capables de décrire l'état d'excitation dans lequel le groupe classe se trouve. L'activité de relaxation } \\
\text { détend l'atmosphère et semble inspirer les jeunes. L'atelier cuisine est riche en échange, les jeunes } \\
\text { doivent coopérer : résoudre des problèmes et exercer leur habileté de communication et ce, durant } \\
\text { de la confection et la présentation des mets. Les parents qui ont été témoins de cet atelier démontrent }\end{array}$ \\
$\begin{array}{l}\text { une satisfaction et un intérêt à l'égard des exploits de leur progéniture et des nouveautés culinaires } \\
\text { présentées. }\end{array}$
\end{tabular}

\section{Présentation des projets intégrateurs}

Les jeunes ont réalisé de merveilleux projets. Toutes les équipes utilisent du support visuel (photos, affiches, maquettes). Leur présentation recèle beaucoup d'informations; les parents semblent enchantés et surpris de la qualité des présentations. Les titulaires de classe considèrent que les sorties en plein air ont permis aux jeunes de ramasser des objets, d'observer des phénomènes et parfois de trouver réponse à leur questionnement.

$\mathrm{Au}$ lever, l'ambiance s'apparente à celle d'un essaim d'abeilles. Chacun est à son affaire, ramasse ses objets personnels et s'installe pour le déjeuner. Tout doit être rangé avant le début des classes. Ils sont fiers et complices. Je sens que le groupe classe vit un sentiment de satisfaction et d'accomplissement.

A10 - Le jour $\mathrm{J}:$ : on couche dehors! »

Environnement social et pédagogique de qualité.

Connaissances sur : l'alimentation;

l'activité physique (bienfaits physiologiques et psychologiques);

l'environnement naturel;

Habiletés : psychomotrices;

interactions sociales;

se fixer des buts et les atteindre;

Comportement sain et sécuritaire.

Satisfaction personnelle.
Environnement social et pédagogique de qualité. Connaissances sur : l'alimentation;

l'activité physique (bienfaits physiologiques et psychologiques).

l'environnement naturel; Habiletés : psychomotrices; interactions sociales; se fixer des buts et les atteindre; Comportement sain et sécuritaire. Satisfaction personnelle.

Observations de la chercheure

Dans l'ensemble, je crois avoir atteint la majorité des intentions éducatives que je m'étais fixée en début de projet. Je sens que les jeunes retirent de l'expérience une grande satisfaction personnelle. L'expédition finale était un défi individuel et de groupe. Cette situation a donné naissance à des moments de plénitude et d'accomplissement personnel. Plusieurs jeunes m'ont témoigné leur appréciation envers l'expérience. J'ai eu l'occasion de les observer dans des situations diverses et mon plus grand constat serait la présence d'un respect mutuel qui laisse sous-entendre l'émergence d'un sentiment d'identité au sein du groupe. 


\subsection{L'éducation à la santé en milieu scolaire favorisant le développement de}

\section{l'estime de soi}

L'estime de soi fait appel à la confiance fondamentale que porte l'être humain envers lui-même (Duclos, 2000) et se traduit comme une perception plus réaliste de son être. L'estime de soi est l'un des plus importants facteurs de protection rapportés dans la littérature (Arcand et al., 1998; Hamel, 2001; Ledoux, 2002) et se construit progressivement à la suite de réalisations porteuses de sentiments positifs.

Les éléments de réponse obtenus à l'aide des outils de collecte de données ont été classifiés selon les axes et sous-axes du DGF Santé et bien-être (tableaux 4.2.1 à 4.2.4). Ces éléments illustrent les principales constituantes du phénomène étudié (Paillé \& Mucchielli, 2008) et dévoilent l'émergence de trois facettes prédominantes: la conscience corporelle, la modification des repères environnementaux exigeant de la coopération, et la prise de conscience sur le rapport avec la nature conduisant vers le désir d'accomplissement.

\subsubsection{Conscience corporelle}

Les grilles d'analyse (tableaux 4.2.1 à 4.2.3) évoquent que l'expression des besoins physiques, de sécurité, d'acceptation et d'épanouissement, ainsi que le besoin d'actualisation émergent davantage au cours des ateliers 2 et 3 qui utilisent 
notamment l'approche sensorielle comme outil didactique, et durant les ateliers 4, 8 , 9 et 10 qui exigent un dépassement personnel sur les plans physique et mental.

La triangulation des méthodes (Karsenti \& Savoie-Zajc, 2004) permet de dégager que les ateliers qui emploient principalement une approche sensorielle (activités d'acclimatation), ou qui proposent des défis personnels, seraient plus favorables à l'éveil des besoins physiques (tableau 4.2.1), à l'acceptation de l'autre et à l'épanouissement personnel et ce, autant chez les garçons que chez les filles (tableau 4.2.3). L'émergence d'une conscience corporelle incite aussi les jeunes participants à se questionner à l'égard du fonctionnement de leur corps ou de phénomènes scientifiques tels que l'étude du vivant et du non-vivant. 
Tableau 4.2.1: Besoins physiques

\begin{tabular}{|c|c|}
\hline $\begin{array}{l}\text { Extraits du journal de bo } \\
\text { Commentaires de la } \\
\text { chercheure-acteure }\end{array}$ & Citations (ou écrits) des jeunes \\
\hline $\begin{array}{l}\text { A9 } \\
\text { Nous voilà en début d'activité } 9 \text {, } \\
\text { les jeunes possèdent } \\
\text { plusieurs notions leurs } \\
\text { signaux corporels semblent } \\
\text { plus aiguisés, l'esprit } \\
\text { d'équipe est installé et ils } \\
\text { s'apprêtent à vivre quelque } \\
\text { chose d'excitant : Le } \\
\text { coucher à l'école. [...] c'est } \\
\text { intéressant, de remarquer } \\
\text { que les malaises (ou besoins) } \\
\text { physiques sont exprimés en } \\
\text { premier par les jeunes. } \\
\text { L'état physique, ensuite } \\
\text { l'état mental } \\
\text { (psychologique) dans lequel } \\
\text { ils se trouvaient fut } \\
\text { mentionné. (Extrait du } \\
\text { journal de bord) }\end{array}$ & $\begin{array}{l}\text { A2 - Q1 : Selon toi, quel lien as-tu avec la nature? } \\
\text { S2 : On a besoin d'eau pour vivre, et de l'air. } \\
\text { S18 : Les arbres respirent et moi aussi. } \\
\text { S23 : J'ai un lien vital avec la nature, quand je respire, quand je bois } \\
\text { de l'eau et quand je mange. } \\
\text { A3 - Q2 : Décris-moi en tes mots la respiration? } \\
\text { S2 : La respiration nous sert à vivre. } \\
\text { S3 : Si on ne respire pas, on meurt. Cela est important. } \\
\text { S7 : On respire de l'air pour vivre, on rejette le gaz carbonique. } \\
\text { S16: La respiration sert à nous faire vivre et à digérer. } \\
\text { A4 - Q2 : Selon toi, l'eau est-elle essentielle pour ton organisme? } \\
\text { S1 : Sans eau, on serait déshydraté (réponse similaire pour S6 - S19- } \\
\text { S24). } \\
\text { S3 : Mon corps est composé de 70\% d'eau, donc j'en ai besoin pour } \\
\text { vivre. } \\
\text { S5 : (l'eau) ça nous hydrate et aussi les muscles vont prendre l'eau } \\
\text { pour nous donner de l'énergie. } \\
\text { S10: L'eau nous donne de l'énergie. } \\
\text { S11 : Si je ne prends pas d'eau, mes muscles n'auront pas d'énergie. } \\
\text { Car pour envoyer l'énergie plus vite dans le sang, il faut de l'eau. } \\
\text { S13 : (l'eau est essentielle) pour l'énergie de notre corps. } \\
\text { S14 : Pour rester en forme, pour s'hydrater. } \\
\text { S15 : Il faut hydrater notre organisme. L'eau est très importante dans } \\
\text { notre corps [...] } \\
\text { S16: (l'eau est essentielle) pour nos muscles. } \\
\text { S18 : L'eau nous aide à grandir. } \\
\text { S21 : Quand on fait de l'activité physique, nos muscles forcent, ils ont } \\
\text { besoin d'eau. }\end{array}$ \\
\hline \multicolumn{2}{|c|}{$\begin{array}{l}\text { A9 (propos spontanés des élèves - extraits du journal de bord) } \\
\text { À la suite d'une série d'exercices vécus à l'extérieur, je remarque l'excitation. Alors avant de rentrer, je } \\
\text { fais un retour. Maintenant, expliquez-moi comment vous vous sentez? L'un me répond : «j'ai les } \\
\text { pieds gelés! », L'autre : «j'ai soif! », puis un troisième mentionne (S16) quelque chose du genre : je } \\
\text { crois que nous sommes excités! (Extrait du journal de bord A-9) }\end{array}$} \\
\hline
\end{tabular}




\subsubsection{Modification des repères}

L’environnement physique suggéré par les ateliers d'aventure en plein air crée en soi de l'inconfort et ébranle la sécurité des jeunes habitués au milieu scolaire conventionnel. Par exemple, la présence potentielle de bêtes sauvages, le changement de température et les grands espaces deviennent pour certains des facteurs insécurisants. Le groupe devient alors synonyme de confort et de sécurité pour les participants (tableau 4.2.2). Les activités d'acclimatation (approche sensorielle) axées sur l'ouverture aux stimulations environnantes, composante 1 de la compétence transversale Structurer son identité contribue à la mise en place d'un climat de confiance (annexe 11 - tableau A). 
Tableau 4.2.2: Besoin de sécurité

Propos des enseignantes aux entrevues finales et extraits du journal de bord - Commentaires de la chercheure-acteure

Enseignante d'éducation physique et à la santé

[...] t'sais le fait de n'avoir vécu d'autres... c'était moins de l'inconnu. Pis entre eux les jeunes se connaissaient. Si quelqu'un est moins « sécure », ben y'a son ami qu'est là [...]T'sais les enfants, comment ça fait. T'as ton ami qu'est là, ben tu vas la (le) chercher comme pour te sécuriser. Pour le projet, c'est génial, la sécurité, tout était là. En plus, à la dernière sortie t'avais des gens de l'UQAC.

Extrait du journal de bord - A10

J'étais en train d'organiser les jeunes qui désiraient coucher à l'extérieur... lorsque j'entre dans le chalet et l'enseignante 2 leur dit : « On est en plein bois, y'a pas d'électricité ici ». T'sais, elle leur donne toutes les mises en garde possibles et inimaginables, puis finit par leur dire : « Si jamais... t'sais... Vous vous sentez mal, vous avez envie de vous confier, Intervenant 5 et moi, on est là... ». À ce moment-là [...] en sourdine j'entendais : « Ben voyons (enseignante 2.), on aura pas peur, inquiète toi pas... on se sent bien [...] ». Ils se sentaient bien, ils se sentaient en sécurité $[. .$.$] il était en gang! J'ai trouvé ça très coquet.$

Au cours de la séquence d'aventure, les titulaires de classe ont observé une réorganisation du groupe en fonction des forces de chacun, la hiérarchisation a été redéfinie (Neill et Richard, 1998). Le journal de bord de la chercheure rapporte que, dès l'atelier 2, les titulaires de classe observent l'émergence de nouveaux leaders. Puis, lors de l'entrevue finale, elles soulignent le développement de la composante contribuer au travail collectif de la compétence 8 (Annexe 11 - tableau D-E et F) et dévoilent que les ateliers $2,5,6,7$ et 9 qui proposent davantage d'activités de communication et de coopération ont contribué à accroître la confiance des jeunes participants.

Les activités de communication et de coopération vécues notamment lors des ateliers 2 et 5 amorcent de nombreux échanges entre les participants et les intervenants scolaires. Tous les intervenants affirment avoir vu naître au cours de 
l'expérimentation- une évolution de l'acceptation mutuelle des pairs (Neill \& Richard, 1998; Hattie et al., 1997) et un climat social porteur de sérénité (tableau 4.2.3). Selon les données recueillies à l'aide des différents outils de collecte de données, la chimie du groupe s'est édifiée grâce à l'évolution graduelle de la séquence d'aventure en plein air. De façon générale, les observations des titulaires de classe, de la chercheure-acteure et des parents vont dans le même sens. Tous estiment que les jeunes ont exprimé des émotions et des sentiments positifs pendant ou à la suite des actions réalisées avec le groupe. Ils ont aussi remarqué que les élèves démontrent un intérêt grandissant pour l'aventure, la nature, l'école et leur bien-être. Ces aspects sont discutés dans une prochaine section de ce chapitre. 
Tableau 4.2.3: Besoin d'acceptation et d'épanouissement comme garçon ou fille

\begin{tabular}{|c|c|c|}
\hline $\begin{array}{l}\text { Extraits du journal de } \\
\text { bord - } \\
\text { Commentaires de } \\
\text { la chercheure- } \\
\text { acteure }\end{array}$ & $\begin{array}{c}\text { Propos des enseignantes } \\
\text { lors des entrevues } \\
\text { finales }\end{array}$ & Citations (ou écrits) des jeunes \\
\hline $\begin{array}{l}\text { A10 } \\
\text { Lors du retour final, le } \\
\text { sujet a mentionné } \\
\text { ceci : « Je sais } \\
\text { aujourd'hui que les } \\
\text { filles sont capables } \\
\text { de faire ce que les } \\
\text { gars sont capables } \\
\text { de faire. » }\end{array}$ & $\begin{array}{l}\text { Enseignante d'éducation } \\
\text { physique et à la santé } \\
\text { Ben, moi aussi, ça me } \\
\text { permettait de mixer les } \\
\text { groupes [...] Je n'ai pas } \\
\text { vu de différence entre } \\
\text { les gars et les filles. } \\
\text { J'pense tout le monde } \\
\text { était à l'aise. Je n'ai pas } \\
\text { senti que les filles } \\
\text { étaient (moins) à l'aise. } \\
\text { Enseignante } 2 \\
\text { S5, le dépassement. S11, } \\
\text { relever des défis, ne pas } \\
\text { lâcher et les filles [...] } \\
\text { elles sont meilleures } \\
\text { qu'elles pensaient. } \\
\text { Enseignante } 1 \\
\text { J'dirais qu'au travers des } \\
\text { activités, il se permettait } \\
\text { plus [...] ils } \\
\text { s'exprimaient plus... }\end{array}$ & $\begin{array}{l}\text { A3-Q3 : Trouves-tu facile de faire confiance } \\
\text { aux autres? } \\
\text { S1 : Dans notre classe, on fait confiance les } \\
\text { uns, les autres. } \\
\text { S7 : Je suis pareille (forte et très vite), il y a } \\
\text { des personnes plus fortes que moi et } \\
\text { d'autres moins fortes. } \\
\text { A8-Q2 : Décris-moi un moment où tu t'es } \\
\text { senti émerveillé, où tu as appris } \\
\text { quelque chose : } \\
\text { S21 : Je me suis émerveillée quand j'étais } \\
\text { seule dans le bois, j'étais dans ma bulle, } \\
\text { j'étais seule et concentrée par le bruit } \\
\text { alentour. J'étais bien. } \\
\text { A10-Q1 : Au cours de l'activité tu as vécu } \\
\text { différentes situations, décris-moi un } \\
\text { moment où tu as pris conscience de ton } \\
\text { corps : } \\
\text { S1 : Je me suis senti en pleine forme et en } \\
\text { santé, en montant au lac. } \\
\text { Commentaires généraux (retour final) } \\
\text { S7 : J'ai beaucoup appris sur mon corps, } \\
\text { comment il est fort. }\end{array}$ \\
\hline
\end{tabular}

\subsubsection{Prise de conscience sur leur rapport avec la nature conduisant vers le désir d'accomplissement}

Pour combler le besoin d'actualisation de soi, l'individu doit connaitre son potentiel, ses habiletés et ses compétences. Ainsi, la connaissance de soi-même devient essentielle. Il est intéressant de constater que certains ateliers (A2, A3, A4, 
A6, A8 et A10) semblent proposer aux jeunes une prise de conscience sur le rapport qu'ils entretiennent avec la nature. Certains écrits des jeunes présentés dans le tableau 4.2.4 suggèrent, dès l'atelier 2 que l'environnement naturel conduit vers une prise de conscience de soi et dévoilent le désir d'accomplissement personnel ou d'actualisation de soi.

Tableau 4.2.4 : Besoin d'actualisation de soi

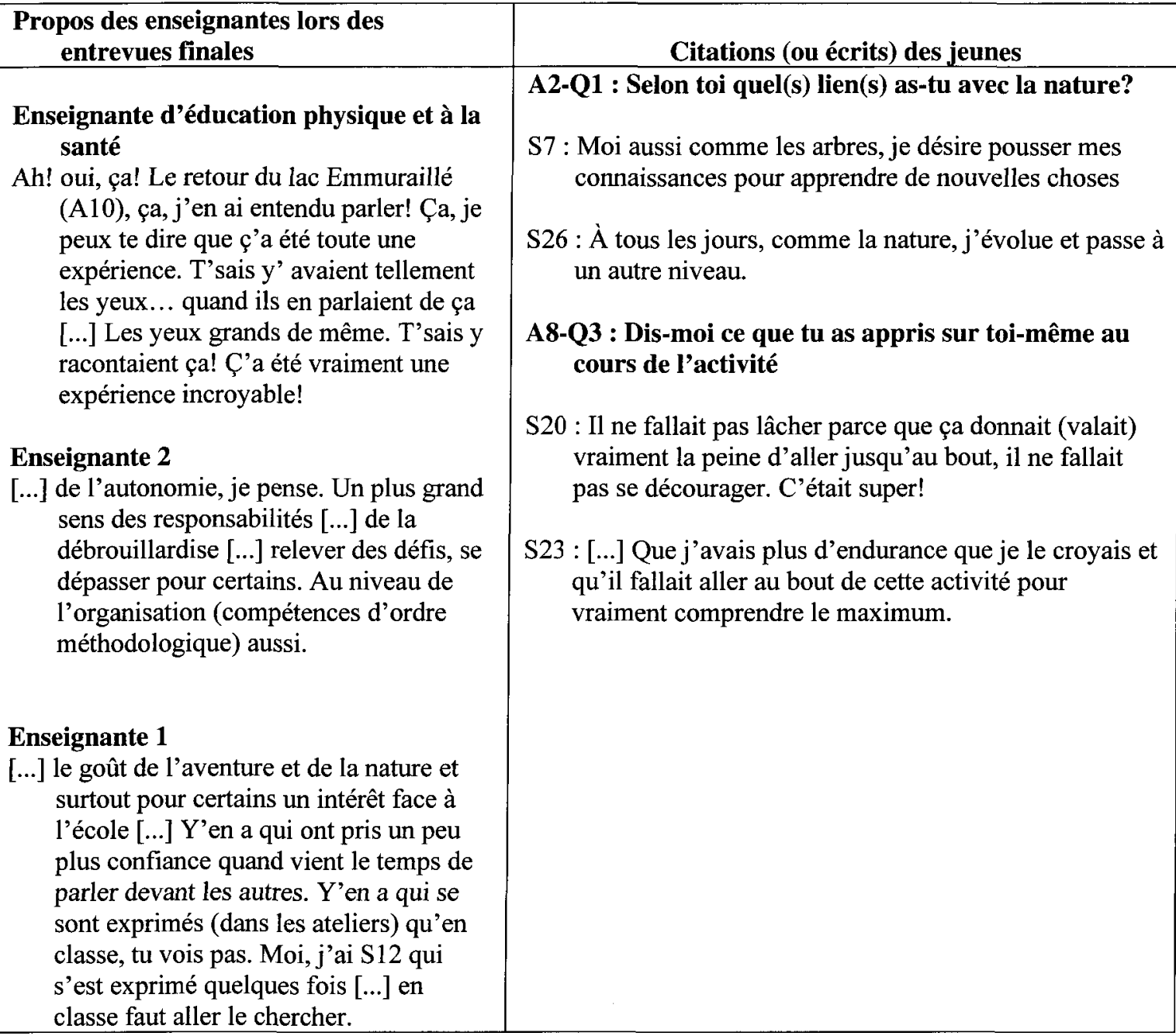


Dans le même ordre d'idées, l'enseignante d'éducation physique et à la santé se souvient d'avoir perçu de la fierté dans les yeux de nombreux élèves, lors du retour du coucher en forêt (l'expédition finale).

Les titulaires de classe estiment que l'expédition finale fut une « expérience de vie incroyable! » Elles considèrent que le défi personnel demandé contribue au développement de plusieurs compétences personnelles et favorise l'émerveillement des jeunes.

De plus, lors du retour final, à l'écrit comme à l'oral, les sujets 10,20,21, 23 et 28 soulignent qu'ils savent maintenant relever des défis, réaliser de «grosses choses ». Le sujet 7 se voit maintenant capable de réussir des épreuves; les sujets 22 et 28 mentionnent qu'ils sont capables de marcher huit kilomètres. Le sujet 24 s'est ainsi exclamé : « je suis fière de mes réalisations! » Ces témoignages qui se trouvent aux tableaux 4.2.5 et 4.2.5.1 évoquent l'expression d'une satisfaction personnelle ou d'un sentiment positif à l'égard de soi-même ou d'un accomplissement personnel. 
Tableau 4.2.5:-Estime de soi - Extraits du journal de bord de la chercheure-acteure et citations (ou écrits) des jeunes

\begin{tabular}{|c|c|}
\hline $\begin{array}{l}\text { Extraits du journal de bord - Commentaires de } \\
\text { la chercheure-acteure }\end{array}$ & Citations (ou écrits) des jeunes \\
\hline $\begin{array}{l}\text { A8 } \\
\text { Avant de traverser le pont, je demande aux jeunes, } \\
\text { en un mot de faire un bref retour sur leur } \\
\text { journée et l'excursion. Dans l'ensemble, ils ont } \\
\text { beaucoup apprécié, ils sont fiers d'eux et ont } \\
\text { adoré les activités collectives. } \\
\text { A9 } \\
\text { Les jeunes sont enchantés de leur expérience, } \\
\text { beaucoup de satisfaction personnelle, il se } \\
\text { dégage un sentiment d'appartenance au } \\
\text { groupe, lorsque avant de quitter le groupe, ils } \\
\text { ont réussi avec succès la chaise humaine. Cette } \\
\text { activité est devenue un incontournable à la fin } \\
\text { de chaque atelier. } \\
\text { A10 } \\
\text { Selon l'enseignante 2, les ateliers auront servi à } \\
\text { leur apprendre la persévérance, la patience, à } \\
\text { développer leur endurance et leur fierté à la } \\
\text { suite d'une réalisation. } \\
\text { Plusieurs jeunes sont simplement venus me voir } \\
\text { pour me démontrer leur reconnaissance. Deux } \\
\text { d'entre eux l'ont démontré plus fortement, ils } \\
\text { sont fiers de leur réalisation et encore plus } \\
\text { parce qu'ils vivent des difficultés personnelles. } \\
\text { S15, S24 et S5 étaient rayonnants. Je sens que } \\
\text { tous les jeunes ont trouvé le moyen de } \\
\text { s'épanouir dans le calme et le respect d'autrui. }\end{array}$ & $\begin{array}{l}\text { L'activité } 3 \\
\text { S5 : J'ai bien aimé cette activité (les exercices les } \\
\text { yeux bandés) ça m'a fait (donné) confiance en } \\
\text { moi. } \\
\text { L'activité } 8 \\
\text { S5 : Confiance en moi, voilà ce que j'ai appris sur } \\
\text { moi-même. } \\
\text { A10 - Q2 : } \\
\text { S1 : J'étais content de moi. } \\
\text { S24 : De la fierté. } \\
\text { S28 : J'étais contente, j'étais fière de moi. } \\
\text { A10 - Q4 } \\
\text { S28 : Métamorphose. } \\
\text { A10 - Q5 } \\
\text { S5 : J'ai pris confiance en moi. } \\
\text { S10 : Que j'étais capable de me rendre loin. } \\
\text { S20 : Que j'étais capable de relever des défis. } \\
\text { S21 : Que je peux relever des gros défis. } \\
\text { S22 : Que j'étais fort pour surmonter } 4 \text { kilomètres } \\
\text { de marche à raquette. } \\
\text { S23 : Que j'étais capable de faire de grosses choses. } \\
\text { S27 : Que je suis capable de marcher huit } \\
\text { kilomètres avec un gros sac à dos. } \\
\text { S28 : Que je suis capable de réaliser des défis. } \\
\text { Retour oral deux semaines après l'expédition } \\
\text { finale, propos transcrits par les titulaires de } \\
\text { classe. (numéro de sujet non mentionné) } \\
\text { Confiance en soi (4); persévérance; ténacité; } \\
\text { débrouillardise (2); maturité (2); sécurité; } \\
\text { meilleure connaissance de soi; capacité de } \\
\text { reconnaître ses faiblesses. }\end{array}$ \\
\hline
\end{tabular}


Tableau 4.2.5.1: Estime de soi - Propos des enseignantes et des parents

\begin{tabular}{l}
$\begin{array}{l}\text { Propos des titulaires de classe - entrevue finale, et de l'enseignante d'éducation physique et à la } \\
\text { santé - entrevue finale. }\end{array}$ \\
\hline Enseignante d'éducation physique et à la santé \\
Ah! oui, ç'a été frappant! Lui, c'est vraiment un exemple concret. Écoute, je me rappelle le premier cours \\
d'éducation physique, y'avait pas son costume. La première chose qu'il m'avait dit : « moi, \\
l'éducation physique, j'peux pas trop en faire parce que j'ai fait des pneumonies, je fais de l'asthme \\
[...] ». T'sais y'avait une grosse barrière. Pis là, tu vois, la sortie de la chute (activité 4) [...] pis les \\
autres (sorties). C'est pas le même ti-gars que j'ai vu au mois d'août. Y'est rendu là (représentation \\
gestuelle), c'est incroyable ça! À la suite de l'activité 4, j'pense qu'y a réalisé que dans le fond: \\
« Cout'donc, j'suis capable de l'faire. Oui, ça me demande un certain effort, mais [...] ». Peut-être \\
y'avait plus d'intérêt? Mais, y'a réalisé qu'y était capable de faire les choses, de surmonter un défi. \\
Peut-être que c'est un p'tit gars, qu'avait jamais été (poussé)? Peut-être au départ, un peu surprotégé? \\
Je l'sais pas! Mais là, il s'est aperçu, qu'y était capable. Ça été vraiment l'fun de le voir évoluer. \\
(entrevue finale)
\end{tabular}

Propos des titulaires de classe lors de l'entrevue finale en réponse à la question : « Avez-vous perçu des changements chez les jeunes dans leur développement, relation ou comportement ou motivation »

S3 : Combler un besoin d'accomplissement.

S4 : Plus d'initiative, plus d'assurance, une meilleure estime personnelle.

$\mathrm{S} 10$ : Capable de réalisation personnelle.

S12 : Amélioration de son assurance personnelle et de sa capacité de s'exprimer en groupe

S14 : Plus d'intérêt pour les activités offertes par l'école, outre qu'académique.

S22 : Amélioration face à son insécurité personnelle - confiance en lui.

S24 et S29 : Contribuer à leur intégration dans le groupe - Selon les enseignantes, le S29 avai,t l'an dernier, un comportement violent qui s'affichait à son arrivée, mais se serait estompé en cours d'année, notamment grâce aux activités d'aventure éducatives.

Commentaires des parents (questionnaire final)

$\mathrm{S} 2:[\ldots]$ je crois que ça lui a donné de l'assurance envers lui-même.

S5 : Cela l'a rendu plus responsable et un peu plus mature. [...] et plus persévérant [...] et plus conciliant.

$\mathrm{S} 7$ : Elle a appris de nouvelles choses auxquelles, elle ne s'attardait pas auparavant [...] se débrouiller en forêt et la respecte $[. .$.

S9: Plus débrouillard, plus de confiance en lui et mieux organisé.

S10: Les ateliers ont eu un impact sur son sens de l'organisation.

S14 : Il a appris à se débrouiller dans la nature et à respecter l'environnement.

S15: Ma fille était motivée à participer à chacune des sorties. Elle a acquis une plus grande confiance en elle. Je la sens capable de prendre des décisions elle-même, de faire des choix. [...] capable de reconnaitre ses capacités physiques et intellectuelles. [...] plus responsable et plus mature [...] amélioration de la complicité avec ses amis (es).

S21 : L'expression de ses émotions, plus débrouillarde, plus réceptive face aux idées des autres tout en sachant exprimer ses idées de façon respectueuse.

S24 : [...] l'attitude de S24 avec les autres élèves de son groupe, sa capacité à prendre sa place. Il a évolué dans sa relation avec ses pairs, sa place dans le groupe.

S28: [...] une meilleure confiance en elle. 
Plus spécifiquement, le sujet 5 exprime à trois reprises au cours de l'expérimentation avoir pris confiance en lui, l'enseignante d'éducation physique et à la santé, les titulaires de classe et même ses parents sont aussi du même avis. Les titulaires de classe semblent avoir également perçu, chez sept de leurs élèves, une évolution des compétences personnelles et sociales. Tandis que plus de huit parents ont inscrit sur le questionnaire final des commentaires divulguant l'impact positif de la séquence sur le développement, le comportement ou les relations interpersonnelles des jeunes participants (tableau 4.2.5.1).

\subsubsection{Manifestation de l'estime de soi}

Les grilles d'analyse développées ont permis de recueillir de nombreux éléments convergents vers une manifestation positive de l'estime de soi des jeunes participants. Tous les outils de collecte de données montrent plusieurs exemples relatant une satisfaction personnelle des élèves et l'émergence de sentiments positifs à l'égard de leurs réalisations (tableau 4.2.5). L'analyse des données indique qu'au cours de la réalisation de la séquence d'aventure et d'apprentissage, les jeunes ont exprimé à plusieurs reprises des émotions traduisant le développement d'un sentiment d'identité, de sécurité ou d'appartenance à leur groupe-classe. De plus, les titulaires de classe ainsi que quelques parents ont même remarqué chez certains élèves une évolution de leur degré d'autonomie et une amélioration de leur confiance personnelle. 
Le tableau 4.2.5.1 expose des exemples cités par les titulaires de classe lors de l'entrevue finale qui vont dans le sens des observations de la chercheure-acteure (tableau 4.2.5) ou des commentaires des parents (tableau 4.2.5.1). Par exemple, elles estiment que le sujet 14 dégage plus d'intérêt pour les activités offertes à l'école, que le sujet 10 est davantage capable de réalisation personnelle grâce à la réalisation de la séquence d'aventure et d'apprentissage. Elles ont noté que le sujet 22 s'affiche maintenant avec plus d'assurance et elles ajoutent que les ateliers d'aventure éducative peuvent contribuer à faciliter l'intégration au groupe (S24 et S29).

Le tableau 4.2.6 expose une classification des ateliers selon les facettes prédominantes mises de l'avant dans la première partie de ce chapitre. La stratégie de recherche utilisée établit que la séquence d'aventure et d'apprentissage réunit plusieurs conditions favorisant l'éveil de la conscience de soi, le rehaussement de l'estime de soi et annonce l'impact positif d'une telle réalisation sur le développement de la compétence sociale au sein du groupe. 
Tableau 4.2.6: Facettes prédominantes des ateliers relativement à la conscience de soi et au développement de l'estime de soi

\begin{tabular}{|c|c|c|c|}
\hline & $\begin{array}{l}\text { Conscience } \\
\text { corporelle }\end{array}$ & $\begin{array}{l}\text { Modification des } \\
\text { repères } \\
\text { environnementaux } \\
\text { exigeant de la } \\
\text { coopération }\end{array}$ & $\begin{array}{c}\text { Prise de conscience } \\
\text { sur leur rapport avec } \\
\text { la nature conduisant } \\
\text { vers le désir } \\
\text { d'accomplissement }\end{array}$ \\
\hline $\begin{array}{c}\text { A1 }- \text { Trésor à la } \\
\text { boussole }\end{array}$ & 宋- & & \\
\hline $\begin{array}{l}\text { A2 - Randonnée et jeux } \\
\text { coopératifs }\end{array}$ & & 次 & 㔖 \\
\hline $\begin{array}{l}\text { A3 - Jeux de sensibilité } \\
\text { corporelle }\end{array}$ & 要 & & 次 \\
\hline $\begin{array}{l}\text { A4 - Randonnée à la } \\
\text { chute }\end{array}$ & $\not x$ & & 况 \\
\hline A5-Défis coopératifs & & 拄 & \\
\hline $\begin{array}{l}\text { A6 - Défis et } \\
\text { dégustation }\end{array}$ & & 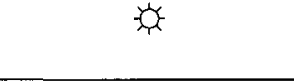 & \\
\hline $\begin{array}{c}\text { A7 - Bouffe et } \\
\text { confiance }\end{array}$ & & 冻 & \\
\hline $\begin{array}{l}\text { A8 - Randonnée } \\
\text { raquette }\end{array}$ & 次 & & 宗 \\
\hline $\begin{array}{l}\text { A9- Coucher intérieur, } \\
\text { bouffe et } \\
\text { présentation }\end{array}$ & & 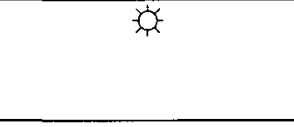 & 荌 \\
\hline $\begin{array}{c}\text { A10 - Le jour J : « on } \\
\text { couche dehors! }\end{array}$ & - & & - \\
\hline \multirow[t]{2}{*}{$\begin{array}{l}\text { Manifestations de } \\
\text { l'estime de soi } \\
\text { selon Martin et } \\
\text { Arcand (2005); } \\
\text { Duclos (2000). } \\
\end{array}$} & $\begin{array}{l}\text { Possibilité d'un } \\
\text { meilleur rapport avec } \\
\text { son identité }\end{array}$ & $\begin{array}{l}\text { Développement } \\
\text { possible de } \\
\text { l'appartenance au } \\
\text { groupe }\end{array}$ & $\begin{array}{l}\text { Satisfaction } \\
\text { personnelle }\end{array}$ \\
\hline & \multicolumn{3}{|c|}{ Compétences diverses } \\
\hline
\end{tabular}




\subsection{Structurer son identité et coopérer}

Les compétences transversales d'ordre personnel et social visent l'affirmation de l'identité du jeune, dans le respect de l'autre (PFEQ, 2001). Structurer son identité et coopérer contribuent au maintien de la santé psychologique de l'individu (Brannon \& Feist, 2007). La stratégie d'analyse des résultats pour cette partie est construite sur l'évolution du développement de ses compétences et de leurs six composantes.

\subsubsection{Structurer son identité : compétence 7}

Structurer son identité s'effectue au moyen d'expériences variées. L'éducation par l'aventure, parce qu'elle suggère la construction de la connaissance dans un environnement naturel, diffère des expériences conventionnelles vécues en milieu scolaire. Berceau d'innombrables stimuli, ce nouvel environnement offre plusieurs occasions de développer l'identité personnelle, sociale, d'élargir les horizons et de mobiliser les facultés (PFEQ, 2001).

Lors d'ateliers vécus en plein air, les jeunes sont constamment exposés à de nouvelles stimulations pour permettre de structurer leur identité, compétence transversale 7. Par exemple, les ateliers d'acclimatation renferment souvent une partie d'exploration sensorielle (ateliers 1, 3 et 8 ) qui suggère aux participants 
l'ouverture aux stimulations environnantes à l'aide de leurs cinq sens : identification de bruits (ou de sons), d'odeurs ou de textures. La réaction à de multiples sensations, l'identification de leurs perceptions et parfois la manifestation ou l'émergence d'une émotion sous-jacente, sont des éléments qui favorisent une ouverture d'esprit à l'égard de soi-même, des autres et de l'environnement (PFEQ, 2001). Les instants vécus en excursions et durant l'expédition sont remplis d'occasions qui permettent aux jeunes de vivre des émotions et d'amorcer des réflexions sur eux-mêmes. Leurs propos suggèrent des manifestations d'émotions agréables et de détente mentale. À quelques reprises, les jeunes ont inscrit sur leur fiche de retour des éléments qui démontrent une curiosité d'esprit par rapport aux stimuli environnants et un accueil aux références morales et spirituelles de leur milieu.

Pour la composante s'ouvrir aux stimulations environnantes, les données recueillies à la suite de la ventilation des résultats se trouvent au tableau 4.3.1 et à l'annexe $11 \mathrm{du}$ tableau A. Le tableau 4.3.1, S'ouvrir aux stimulations environnantes : exemples et manifestations, présente un des nombreux exemples de mise en situation proposée aux jeunes participants, tandis que la colonne de droite énumère quelques réactions écrites des jeunes envers trois des manifestations de la composante ciblée. 
Tableau 4.3.1 : S'ouvrir aux stimulations environnantes $:$ exemples et manifestations

\begin{tabular}{|c|c|}
\hline \multicolumn{2}{|l|}{ Exemples de mise en situation : } \\
\hline \multicolumn{2}{|c|}{$\begin{array}{l}\text { Ils sont loin! Ils sont proches! Ils sont forts! Ils sont sourds! Cherchez à comprendre d'où ils } \\
\text { proviennent. Identifiez-moi, un bruit près de vous...je laisse écouler un peu de temps [...] Identifiez- } \\
\text { en un loin de vous [...]. Un très sourd [...] grave. [...] En deuxième partie, les yeux fermés, les jeunes } \\
\text { sont invités à goûter les fruits du vinaigrier. La consigne : « Identifiez les sensations gustatives } \\
\text { perçues; acide, sucré, rugueux [...] ». Tous ont bien participé, ils étaient concentrés et attentifs! } \\
\text { (extraits du journal de bord, atelier } 8 \text { ) }\end{array}$} \\
\hline $\begin{array}{l}\text { Manifestations de la } \\
\text { composante }\end{array}$ & Propos des jeunes recueillis lors des ateliers 8 et 10 \\
\hline $\begin{array}{l}\text { Réagir aux faits, aux situations ou } \\
\text { aux événements }\end{array}$ & $\begin{array}{l}\text { Il fallait aller dans le bois et entendre les bruits aigus, graves, etc. } \\
\text { Là, j'ai vraiment compris que quand on est dans la forêt, il y a } \\
\text { beaucoup de bruits très différents que quand, par exemple, } \\
\text { quelqu'un habite près d'une autoroute (S22). Moi, quand je } \\
\text { suis rentré dans le chalet pour me coucher, j'ai ressenti la } \\
\text { chaleur du feu se rendre sur mon corps. J'étais très bien (S18). }\end{array}$ \\
\hline $\begin{array}{l}\text { Identifier ses perceptions, ses } \\
\text { sentiments, ses réflexions à leur }\end{array}$ & $\begin{array}{l}\text { Dans la nature... Il y a des bruits d'animaux et de l'eau qui coule } \\
\text { (S1). } \\
\text { [...] j'étais seul et concentré par le bruit alentour, j'étais bien } \\
\text { (S21). } \\
\text { J'ai ressenti : } \\
\text { l'épuisement (S8); l'intrigue (S12); l'émerveillement (S20); } \\
\text { l'impatience (S21); la fierté (S24); la plénitude (S28). } \\
\text { Quand j'ai marché en raquettes, pour me rendre au lac } \\
\text { Emmuraillé, j'étais fatiguée, mais en même temps heureuse } \\
\text { (S15; S17). } \\
\text { Quand je me suis couchée, j'étais contente de ma très belle } \\
\text { journée avec toutes les activités et aussi très excitée de savoir } \\
\text { ce qu'on va faire demain, en plus j'étais avec mes amies. } \\
\text { (S7); nous avons vécu une euphorie...d'être entre ami(e)s } \\
\text { (S10 et S22). } \\
\text { (À la suite de l'expédition-effort physique) [...] j'étais vraiment } \\
\text { très détendue, j'avais chaud et j'étais très calme (S28). } \\
\text { J'étais heureuse parce que c'était la plus belle expérience de toute } \\
\text { ma vie. J'ai ressenti la joie de vivre (S21). }\end{array}$ \\
\hline $\begin{array}{l}\text { Accueillir les références morales et } \\
\text { spirituelles de son milieu }\end{array}$ & $\begin{array}{l}\text { Je me suis émerveillé quand j'étais seul dans le bois. J'étais dans } \\
\text { ma bulle, j'étais concentré sur les bruits autour (S21). }\end{array}$ \\
\hline
\end{tabular}

Le tableau 4.3.2 présente la composante, Prendre conscience de sa place parmi les autres, de la compétence Structurer son identité. Cette composante est étroitement liée à la confiance que le jeune se porte en situation de relations interpersonnelles (MEQ 2001). Élaborer ses opinions et définir ses choix dans 
l'accomplissement d'une tâche ou d'un projet ne semble pas toujours facile, tout comme manifester une ouverture d'esprit envers les valeurs ou aux idées d'autrui.

La perception du jeune à l'égard de ses propres valeurs, la confiance qu'il a en ses capacités (habiletés et compétences) influencent nécessairement ses réactions. Dans son ensemble, la séquence d'aventure et d'apprentissage aura permis de tisser des liens interpersonnels et d'approfondir certaines valeurs de référence des participants. Des citations évoquent même que des élèves plus timides se sont impliqués et exprimés davantage en groupe. À ce sujet, l'annexe 11 présente au tableau B davantage opinions et réflexions des participants relativement à deux des manifestations de la composante Prendre conscience de sa place parmi les autres. 
Tableau 4.3.2 : Prendre conscience de sa place parmi les autres : manifestations et propos

\begin{tabular}{|c|c|}
\hline Manifestations de la composante & Propos des jeunes \\
\hline $\begin{array}{l}\text { Se faire confiance; reconnaître la } \\
\text { valeur de ses pairs }\end{array}$ & $\begin{array}{l}\text { Ce sont mes ami(e)s et je leur fais confiance }[. . .] \text { c'est comme } \\
\text { mes frères et mes sœurs (S2). Je savais qu'ils ne me } \\
\text { laisseraient jamais tomber, alors je leur faisais confiance } \\
\text { (S15) (Atelier 7). } \\
\text { J'ai appris : } \\
\text { [...] que j'étais capable de me faire confiance (S17); [...] que } \\
\text { je pouvais être sérieux (S29) (retour atelier 10). }\end{array}$ \\
\hline $\begin{array}{l}\text { Reconnaître son appartenance à une } \\
\text { collectivité }\end{array}$ & $\begin{array}{l}10 \text { des } 27 \text { sujets ayant participé à l'expédition (A10) ont } \\
\text { inscrit sur leur fiche de retour que le fait d'être ensemble } \\
\text { leur a fait vivre une émotion positive. } \\
\text { Ex. : Le fait d'être avec mes amies et vivre quelque chose que } \\
\text { je n'aurais peut-être pas (jamais) vécu un jour m'a fait du } \\
\text { bien (S17). La chaleur du feu qui me réchauffait et de } \\
\text { coucher à l'extérieur de chez nous avec nos amis (S18), } \\
\text { m'a rassuré. }\end{array}$ \\
\hline \multicolumn{2}{|c|}{ Propos de l'enseignante d'éducation physique et à la santé (entrevue finale): } \\
\hline \multicolumn{2}{|c|}{$\begin{array}{l}\text { [...] lui j'peux dire que ça été super bon [...] même (dans) sa relation avec les autres. T'sais au départ } \\
\text { les autres avaient tendance à pas trop le vouloir dans leur équipe, mais à la fin, y'était intégré }[\ldots] \\
\text { ouais. }\end{array}$} \\
\hline \multicolumn{2}{|c|}{$\begin{array}{l}\text { Commentaires de titulaires de classe - inscrits dans le journal de bord à la suite du retour à mi-parcours } \\
\text { (A6) }\end{array}$} \\
\hline \multicolumn{2}{|c|}{$\begin{array}{l}\text { J'ai apprécié voir évoluer les jeunes dans un autre contexte que la classe. J'ai appris à découvrir } \\
\text { l'enseignement sous un autre angle (enseignante } 2 \text { ). }\end{array}$} \\
\hline \multicolumn{2}{|c|}{ Propos de parents (questionnaire): } \\
\hline \multicolumn{2}{|c|}{$\begin{array}{l}\text { [...] plutôt avec les autres élèves dans l'attitude en groupe, sa capacité à prendre sa place. J'ai perçu une } \\
\text { évolution dans son acceptation dans le groupe (S24). } \\
\text { [...] " Nouveau concept » pour apprendre à se connaître et vivre de nouvelles expériences à l'extérieur } \\
\text { de l'école avec ses amis, enseignants [...] (S15) }\end{array}$} \\
\hline
\end{tabular}

Les grandes manifestations de la composante, Mettre à profit ses ressources personnelles (PFEQ, 2001) se résument à la manifestation de plus d'autonomie et d'indépendance; à la capacité d'exploiter ses forces et de ses limites et de juger de la qualité et de la pertinence de ses choix d'actions. Ces facteurs favorisent la consolidation de l'estime personnelle du jeune (voir les tableaux 4.2.5 et 4.2.5.1). 
Les nombreux échanges avec les enseignantes nous portent à croire que la séquence d'aventure et d'apprentissage développe, de façon générale, l'autonomie et la débrouillardise chez les jeunes. Selon elles, l'éducation par l'aventure possède un potentiel sans équivoque sur le développement des élèves, en lien avec cette composante. L'enseignante 2 mentionne à ce sujet :

Oui y'a eu des gains, pis oui, ils vont y penser face à une autre difficulté et c'est plusieurs projets comme ça qui vont amener un enfant à se développer (Enseignante 2).

Les titulaires de classe et l'enseignante d'éducation physique et à la santé considèrent par ailleurs que la manifestation de cette composante s'est faite graduellement (annexe 11, tableau C). Selon ces intervenants scolaires, la planification de la séquence d'aventure a permis aux élèves de réaliser des ateliers plus exigeants et de dépasser leurs limites en mettant à profit leurs ressources personnelles. Voici la réflexion que porte l'enseignante d'éducation physique et à la santé à l'égard d'un des participants concernant ce sujet :

La première sortie (A4 - randonnée à la chute). Ben, j'vois S5! (Le défi) il n'était pas si gros [...], mais pour lui, ça devait être gros, pis tu vois y'a été capable de faire la dernière sortie (qui était un défi personnel plus important), le coucher en forêt [...] t'sais ça y'a permis de voir [...] oui c'est difficile, mais: "hop! J'ai passé au travers, je suis capable d'évoluer là-dedans».

Au fil des ateliers, les jeunes en sont venus à prendre des risques. Par exemple, les sujets $8,9,10,11,13$ ont réalisé qu'ils étaient capables de marcher une longue 
distance. Le sujet 12 note qu'il a appris que lorsqu'il veut réaliser quelque chose, il le peut : «[...] si je veux, je peux!» a-t-il écrit. D'autres ont réalisé qu'ils sont capables de relever des défis (S7 et S9) avec un gros sac sur le dos (S4 et S18) et ce, sur une longue distance (S12 et S16). La classification des éléments constructifs recueillis à l'aide des grilles d'analyse traduit plusieurs manifestations de cette composante. L'arbre thématique construit évoque que la réalisation de la séquence d'aventure et d'apprentissage favorise le développement de la composante Mettre à profit ses ressources personnelles sous plusieurs angles.

\subsubsection{Coopérer : compétence 8}

Les ateliers proposent des mises en situation qui obligent les élèves à interagir avec ouverture d'esprit dans différents contextes, car la réalisation de petits défis d'aventure exige généralement une contribution collective, sans quoi le but fixé ne peut être atteint. Les réalisations collectives telles que le projet intégrateur, les préparations culinaires et l'ensemble du cheminement menant à l'expédition au lac Emmuraillé, en sont des exemples. La catégorisation des données recueillies (Bardin, 2007) et la triangulation des sources (Paillé \& Mucchielli, 2008; Karsenti \& SavoieZajc, 2004) ont contribué à découvrir que la réalisation de la séquence d'aventure et d'apprentissage possède un potentiel étonnant pour développer la compétence transversale 8: Coopérer (Annexe 11, tableau D à F). Cette compétence tirée du PFEQ fait référence à la compétence sociale soulevée par Martin et Arcand (2005) dans le guide-École en santé (annexe 1). 
Les titulaires de classe, l'enseignante d'éducation physique et à la santé et la chercheure-acteure ont toutes observé une amélioration de la collaboration au sein du groupe. Aussi, certains parents ont mentionné avoir perçu des changements dans l'attitude de leur enfant envers la relation qu'il entretient avec les autres élèves du groupe. À ce sujet, au moins trois parents ont mentionné trouver leur enfant plus réceptif et respectueux envers les idées des autres (Annexe 11, tableau D).

Les paragraphes qui suivent présentent quelques perceptions et commentaires recueillis par les outils de collecte de données qui démontrent une situation de coopération en évolution dès l'atelier 1 :

Pour arriver à découvrir la boîte cachée, ils devaient travailler en équipe. Une équipe a tardé à trouver le premier azimut, car ces derniers essayaient de résoudre le problème de façon individuelle. Lorsqu'ils se sont décidés à travailler en équipe, ils ont rapidement trouvé le trésor caché. (Extrait du journal de bord-Annexe 11, tableau E)

La situation présentée ci-dessus mentionne que certaines équipes ont eu plus de difficulté que d'autres à coopérer, mais toutes ont finalement réussi le défi proposé. Ceci n'est qu'un exemple d'atelier d'apprentissage demandant de la coopération: l'étude de l'écosystème (A2), le projet d'intégration (A4 à A9), les défis coopératifs (A5), l'étude d'une recette et sa préparation (A7 à A9), l'expédition finale (A10) sont tous des exemples de situations qui ont demandé aux jeunes une participation collective. 
En outre, la collaboration, l'entraide et l'apprentissage du travail en collaboration font partie des éléments marquants de l'expérience, selon les jeunes participants. À titre d'exemple, à mi-parcours, les sujets 7 et 26 disent avoir appris l'entraide en équipe, tandis qu'à la fin du projet, lorsque nous leur avons demandé de décrire en un mot ce qu'ils ont vécu, 5 des 29 participants, soit environ $20 \%$ des jeunes, ont répondu spontanément : coopération et entraide (Annexe 11 - tableau F). La séquence d'aventure et d'apprentissage a permis aux élèves de se conscientiser et de développer des apprentissages en matière de coopération.

L'analyse des résultats évoque que les activités de résolution de problèmes (ateliers $2,5,7,8,9$ et 10 ) créent des situations interactives conduisant les jeunes à des échanges interpersonnels. L'atelier 2, dont l'un des objectifs est de sensibiliser les participants à l'importance de l'entraide et de la collaboration avec autrui et avec l'environnement, en est un bon exemple. Précédée d'une situation d'apprentissage et d'évaluation sur l'écosystème et l'interdépendance des êtres vivants (tirée de Toussaint \& Lavigne, 2001), la fiche de retour porte sur la collaboration (annexe 6). L'analyse des trois tableaux ayant servi à la classification des données, propose que plus le nombre d'ateliers réalisés est grand, plus les réflexions et commentaires sont éloquents. Le développement de la compétence Coopérer s'effectue progressivement et les élèves cheminent en ce sens. L'éveil commence à l'atelier 2 comme le démontrent les commentaires de S6 et S17 qui soulignent que la collaboration permet l'échange d'idées et d'opinions; S23 soutient que collaborer garde la bonne humeur et le sujet 20 considère qu'il faut s'entraider pour réussir notre vie. Aussi, les 
sujets 4 et 16 croient que, si l'on ne collabore pas, on n'a pas d'amis (es), tandis que les sujets 9 et 11 estiment que pour réussir quelque chose, on a besoin des autres. Finalement, le sujet 15 juge la collaboration primordiale pour la survie de 1'humanité, pour vivre et pour grandir (Annexe 11 - tableau D).

Bien que dès le deuxième atelier les jeunes ont été invités à réfléchir sur le sujet, ce n'est qu'à la fin du quatrième atelier que les intervenants scolaires ont perçu certaines manifestations des composantes de la compétence Coopérer. Il est à noter qu'au cours de l'excursion (A4), les jeunes commencent la cueillette d'informations pertinentes pour la réalisation du projet intégrateur. Ainsi, à la suite de cet atelier, les jeunes doivent travailler ensemble à la réalisation de leur projet qui se déroule sur une période de quatre mois, soit de novembre à février. Selon les titulaires de classe, les jeunes démontrent, durant les périodes de travail collectif en classe, une grande motivation à l'égard de la planification de ce projet. Elles observent aussi que les élèves profitent de chacune des sorties ultérieures, pour recueillir des informations pertinentes; consolider le tout et créer leur présentation (A9). Voici le propos de l'enseignante 2 au sujet de la réalisation du projet intégrateur :

Beaucoup de motivation parce que c'était lié directement au projet (éducation par l'aventure). Le choix s'était fait en sortie, pis dans les autres sorties, ils accumulaient des informations sans compter les informations recueillies dans des livres et sur le Web qui contribuaient à enrichir leur projet. 
Selon les titulaires de classe, chacun des jeunes participants a contribué, à sa façon, au travail collectif et a accompli sa tâche selon les règles qu'ils avaient préalablement établies. L'analyse des résultats révèle que la composante tirer profit du travail en coopération s'est davantage concrétisée dans la réalisation du projet intégrateur, puisque les jeunes ont dû identifier les tâches à réaliser, amasser des informations et concevoir la présentation finale.

D'autre part, la réalisation de la séquence d'aventure et d'apprentissage, dans sa totalité, a influencé le développement de cette composante, car l'accomplissement de plusieurs tâches individuelles influence l'atteinte de l'objectif collectif, c'est-àdire la réalisation des recettes (A9), la préparation de l'expédition (A10), etc. Pour S1, l'esprit d'équipe l'habite au point que seul face à sa fiche de retour il inscrit : «c'était froid, et on a lutté tous ensemble! ».

En effet, plusieurs intervenants scolaires ont identifié une évolution des attitudes et des comportements des jeunes indiquant des actions d'entraide et de coopération (Annexe 11 - tableau F) :

Le fait d'encourager les autres. T'sais même si quelqu'un a plus de difficulté [...] ben t'sais: "on lâche pas!" (Enseignante 1) [...] les jeunes allaient aider les autres à arranger leur sac. C'était beau! (Entrevue finale propos de la suppléante qui a accompagné le groupe lors de l'expédition finale). 
C'est ça au fur et à mesure, j'pense qu'ils se sont en allés vraiment comme ça $[. .$.$] Ça (la séquence d'aventure et$ d'apprentissage) construit de meilleurs liens [...] plus de coopération [...] Ça forme un groupe plus homogène! (enseignante d'éducation physique et à la santé- entrevue finale)

La mise en place d'une séquence d'aventure et d'apprentissage nécessite la coopération des acteurs du milieu. Par conséquent, les forces de tous les jeunes conjuguées à celles des intervenants scolaires sont primordiales pour réaliser un tel projet. Sans coopération des acteurs du milieu, l'introduction d'un atelier d'éducation par l'aventure est impossible. Les données recueillies indiquent que l'environnement social créé par la réalisation de la séquence d'aventure et d'apprentissage, combiné au soutien de la direction de l'école, du conseil d'établissement et des parents suscite l'ouverture d'esprit des jeunes envers les apprentissages, stimule leur désir d'accomplir les tâches collectivement et d'apprécier la participation de tous pour enrichir le travail accompli. D'ailleurs, un des jeunes participants remarque que la complémentarité des habiletés relationnelles (de communication, de résolution de problèmes, l'exercice du jugement critique, la gestion du stress, la cible et l'atteinte de buts communs) de ses coéquipiers facilite la réalisation des activités en contexte naturel (Annexe 11 - tableau F). Reasoner (1982) souligne que l'appréciation des différences individuelles développe le sentiment d'identité et conduit vers l'amélioration de l'estime personnelle. 


\subsection{Habitudes de vie et comportements sécuritaires}

Au cours de la séquence d'aventure et d'apprentissage, les jeunes ont acquis, par l'action, des connaissances multiples. L'analyse des résultats tend à démontrer que le chevauchement planifié des activités d'apprentissage en classe et des ateliers en plein air, que la cohésion des intervenants scolaires, ainsi que le support de la direction, des parents et du conseil d'établissement ont contribué à l'émergence de multiples facteurs de développement en matière de santé (Martin \& Arcand, 2005). La section qui suit abordera l'adoption de saines habitudes de vie et de comportements sécuritaires. Afin de procéder à l'analyse de ces deux thématiques, la classification des résultats a été orchestrée selon deux des axes et sous-axes du DGF Santé et bien-être: Conscience des conséquences sur sa santé et son bien-être, de ses choix personnels et mode de vie actif et conduite sécuritaire (PFEQ, 2001).

\subsubsection{Habitudes de vie}

La triangulation des données recueillies à l'aide des outils de collecte de données indique que la réalisation de la séquence d'aventure et d'apprentissage influence l'acquisition de connaissances en matière de santé. Certains témoignages livrés par les jeunes et les actions observées par les intervenants scolaires indiquent parfois l'atteinte de savoirs essentiels liés à la troisième compétence du domaine du

développement personnel, éducation physique et à la santé. La catégorisation des résultats révèle que les sujets auraient acquis des connaissances sur les bienfaits 
psychologiques, physiologiques et sociaux de l'adoption d'un mode de vie sain, en matière de gestion du stress, de relaxation et d'hygiène corporelle; et aussi certaines habiletés liées au maintien de la condition physique : flexibilité, posture, endurance cardiovasculaire et vigueur musculaire.

L'analyse des résultats démontre en ce sens que plusieurs élèves ont pris conscience au cours de l'action des conséquences de leur choix personnel sur leur santé et leur bien-être, tantôt, sous forme de questionnement, tantôt, sous forme de prise de conscience. Par exemple, à la question : «Qu'as-tu appris au cours du projet Nature et santé? », les jeunes ont tenu, pour la majorité, des propos en lien avec la conscience de leurs choix personnels sur leur santé et leur bien-être (tableau 4.5.1). Par exemple : aller jouer dehors, respirer et bien manger.... Ainsi, l'évolution de la séquence d'aventure et d'apprentissage a un impact sur l'assimilation des différents concepts en lien avec les habitudes de vie: l'alimentation, l'activité physique, la gestion du stress.

\subsubsection{Alimentation}

L'analyse des grilles thématiques montre une évolution des choix alimentaires des jeunes participants (tableau 4.4.1). Ils semblent avoir acquis des connaissances sur la composition des aliments et leur fonction physiologique. À titre d'exemple, les intervenants scolaires ont noté lors des ateliers 8 et 10 que les lunchs des élèves se composaient davantage d'aliments sains. Leurs commentaires suggèrent que les 
jeunes ont remplacé certains aliments, par exemple, les chips et le chocolat par des fruits ou des barres tendres. Un autre exemple, en période d'activité d'endurance (excursions et expédition), les jeunes semblent de plus en plus donner la priorité à l'eau.

L'enseignante d'éducation physique et à la santé estime que, petit à petit, les jeunes ont effectué des apprentissages en matière d'alimentation, car ils ont eu l'occasion de mettre leurs connaissances en application lors d'activités ultérieures. 
Tableau 4.4.1 : Alimentation

\section{Extraits du journal de bord - Commentaires de la chercheure-acteure et propos des enseignantes lors des entrevues finales \\ Enseignante d'éducation physique et à la santé \\ [...] Même au niveau de l'alimentation [...] t'apportes de « cochonneries », bien tu te rends compte que ça n'apporte rien, alors tu fais attention à la prochaine sortie [...]. Je te dirais que le discours a changé. Comme des fois, ils pouvaient passer des messages, sans s'en rendre compte : «Ah! T'sais ça... telle affaire [...] » À la sortie de... la grosse sortie (A10) [...] ben au niveau de l'alimentation, pis pour en avoir parlé après avec Enseignante 2, pis Enseignante 1, les élèves semblaient bien avoir respecté ce qu'ils devaient avoir comme bouffe.}

\section{Extrait du journal de bord- A4}

L'heure de la collation. J'en profite pour leur donner quelques notions sur les glucides : l'énergie nécessaire pour les muscles.

[...] Les fruits sont la première source d'énergie pour les muscles, que les barres tendres sont bien parce qu'elles contiennent des céréales et souvent des noix, mais que les chips et le chocolat friandise peuvent nous mettre à plat. [...] J'observe leur lunch. Je remarque la présence de +++ pain blanc, jambon, boissons, chips, chocolat $[\ldots]$.

\section{Extrait du journal de bord- $A 8$}

On bouffe!!! Certains d'entre eux examinent le lunch des autres. Après un survol, $j$ 'évalue que dans l'ensemble les lunchs sont plus complets et mieux équilibrés qu'à la première sortie.

Un jeune fait remarquer à Enseignante 2 que sa barre de chocolat friandise n'est pas un bon choix. Je souris et lui laisse expliquer pourquoi!

Extrait du journal de bord- A9

Lors de la dégustation, plusieurs n'ont pas aimé les canneberges,...trop acides..., ils l'ont trouvé euxmêmes. Bravo! Votre goût se développe. [...] Miso, couscous, canneberges... de nouveaux aliments. Les jeunes ont vécu une exploration gustative! Le premier essai, pas toujours facile. Dans ce genre de situation, goûter est fondamental. Après, tu décides si tu aimes ou non! La majorité ont relevé le défi.

\section{Citations (ou écrits) des jeunes}

\section{Retour oral à mi-parcours}

S25 : J'ai appris que l'eau était importante pour la digestion et pour notre vie

S3 : J'ai découvert de la nouvelle nourriture.

S27 : Qu'il y avait deux sortes de chocolat, le vrai et celui des friandises [...]

Retour oral à la fin du projet

S5 : Je connais plus de champignons, la bannique et comment cuisiner des nouvelles recettes.

S15 : J'ai découvert de nouveaux aliments et de nouvelles techniques pour cuisiner.

Ce que je vais améliorer - propos recueillis dans le compte-rendu demandé par les enseignantes à la suite de l'activité 10

S2 : Je vais plus prendre de l'eau parce que lors de la montée, j'ai eu beaucoup chaud.

S14 : [...] Je devrais améliorer c'est manger des bonnes choses comme des fruits : des pommes, des bananes, des oranges et aussi des légumes comme des fèves, etc.

S16 : J'ai découvert de nouveaux aliments, j'en ai goûtés et je sais aussi maintenant qu'on peut manger certaines branches d'arbre.

Données recueillies en lien avec la compétence 3 du domaine personnel : éducation physique et à la santé

Mon corps prend beaucoup de place et mon corps à besoin d'eau pour avoir de l'énergie (S11).

J'ai appris que nos muscles avaient besoin d'eau (S16). 
Les écrits et gestes des élèves démontrent une évolution des connaissances en matière d'alimentation. Les commentaires des titulaires de classe, de l'enseignante d'éducation physique et les perceptions inscrites au journal de bord de la chercheureacteure abondent dans le même sens : la réalisation de la séquence d'aventure et d'éducation par l'aventure en plein air propose aux jeunes de multiples occasions d'effectuer des découvertes alimentaires et d'approfondir leurs connaissances en la matière. À titre d'exemple, l'atelier 7 leur propose une recherche en classe sur de nouveaux aliments. Les thèmes de l'hydratation, les collations riches en glucides (fruits, barres tendres...) pour entretenir l'énergie musculaire, la manifestation de l'hypoglycémie, l'ingestion d'aliments gras et la vitesse de digestion, par exemple un lunch, sont des sujets abordés en atelier, principalement lors d'excursions (A4-A8).

Ainsi, la réponse physiologique $\mathrm{du}$ corps induit le questionnement $\mathrm{du}$ participant et la poursuite des ateliers favorise la mise en application des nouvelles connaissances. Graduellement, les jeunes intègrent certains concepts inscrits à titre de savoirs essentiels des domaines de la mathématique, de la science et de la technologie (tableau 4.4.1 et 4.5.1, p 161). À titre d'exemple, une conscientisation du phénomène de sudation et de thermorégulation: "Je vais plus prendre de l'eau, parce que lors de la montée, j'ai eu beaucoup chaud» (S2).

Finalement, lors de l'atelier 9 (le coucher à l'intérieur), les réalisations culinaires ont créé une synergie au sein du groupe. Chacun a mis la main à la pâte et présenté (en petit groupe) sa mixture au reste du groupe qui a dégusté. Ainsi, lors du 
retour, plus de $15 \%(5 / 29)$ des élèves ont mentionné avoir découvert de la nouvelle nourriture, de nouveaux aliments.

\subsubsection{Activité physique}

La séquence d'aventure met à profit toutes les occasions de bouger et de développer des habiletés de souplesse, de force, de coordination et d'endurance. La littérature rapporte que dès l'enfance, la pratique régulière d'activité physique favorise la santé (Sallis \& Owen, 1999) et la réussite scolaire. Car en plus d'être associée à une meilleure estime de soi (Thibault, 2000; Martin \& Arcand, 2005), elle vise l'amélioration de la concentration.

Les ateliers 2, 4, 8 et 10 ont demandé pour leur réalisation de la force et de l'endurance, tandis que les ateliers 1, 3, 5, 6 et 9 ont exigé le déploiement d'habiletés physiques comme l'équilibre, la souplesse ou la dextérité. Selon les observations de l'enseignante d'éducation physique et à la santé, les jeunes ont appris, au moyen de la séquence d'aventure et d'apprentissage, à doser leur effort et varier plusieurs habiletés pour faciliter la pratique d'activités physiques.

La séquence d'aventure et d'apprentissage permet aux jeunes d'effectuer une prise de conscience personnelle par rapport à la pratique d'activité physique, l'endurance cardiovasculaire, le dosage d'effort, la posture et la respiration. Presque tous ces éléments sont des savoirs essentiels ciblés par la troisième compétence du 
domaine du développement personnel Éducation physique et à la santé. Le tableau

qui suit présente quelques propos des jeunes et les observations des intervenants

scolaires à l'égard de la pratique d'activités physiques.

Tableau 4.4.2: Activité physique

\begin{tabular}{|c|c|}
\hline $\begin{array}{l}\text { Extraits du journal de bord - } \\
\text { Commentaires de la } \\
\text { chercheure-acteure et propos } \\
\text { des enseignantes lors des } \\
\text { entrevues finales }\end{array}$ & Citations (ou écrits) des jeunes \\
\hline $\begin{array}{l}\text { Enseignante d'éducation physique } \\
\text { et à la santé } \\
\text { L'endurance physique, par exemple, } \\
\text { tu peux avoir une faiblesse aux } \\
\text { jambes, pis tu continues pareil. } \\
\text { Pis à la fin t'es [...], car ils l'ont } \\
\text { dit [...] t'es fière de toi parce que } \\
\text { t'es allée jusqu'au bout [...]. La } \\
\text { respiration aussi [...] importante } \\
\text { pour récupérer rapidement! } \\
\\
\text { Extrait du journal de bord - A8 } \\
\text { L'esprit d'équipe s'installe. } \\
\text { Rapidement, je revois avec eux } \\
\text { des notions sur la respiration, le } \\
\text { positionnement, l'alimentation et } \\
\text { la cadence à maintenir pour une } \\
\text { activité d'endurance. }\end{array}$ & $\begin{array}{l}\text { Ce que je vais améliorer - propos recueillis dans le compte- } \\
\text { rendu demandé par les enseignantes à la suite de l'activité } \\
\mathbf{1 0} \\
\text { S5 : Je devrais améliorer mon effort physique pour une autre } \\
\text { randonnée, parce qu'il faut toujours que je m'arrête puisque } \\
\text { je ne fais pas beaucoup de sport. } \\
\text { S14 : Je devrais améliorer ma respiration, car je manque d'air très } \\
\text { facilement et j'ai de la difficulté à respirer. Deuxièmement, je } \\
\text { devrais améliorer ma ficelle (mon positionnement), car j'ai } \\
\text { toujours le dos croche et j'ai de la difficulté à monter dans les } \\
\text { collines très abruptes. } \\
\text { S17 : Il faudrait que je marche plus rapidement (avec un rythme), } \\
\text { car je prenais beaucoup de pauses inutiles qui me faisaient } \\
\text { perdre de l'énergie. } \\
\text { S27: J'aimerais améliorer les montées. Parce que quand je } \\
\text { monte, il faut que j'arrête le moins possible pour moins me } \\
\text { fatiguer. C'est la même chose pour les descentes. }\end{array}$ \\
\hline \multicolumn{2}{|c|}{$\begin{array}{l}\text { Retour oral à la fin du projet } \\
\text { J'ai appris le positionnement (S5) } \\
\text { Se tenir droit et prendre de bonnes respirations aide quand on monte quelque chose (S6) } \\
\text { J'ai appris à me tenir droit pour avoir plus de facilité à marcher (S19) } \\
\text { Ma position quand je marche, comment respirer correctement (S21) } \\
\text { Apprentissage de l'exercice de respiration (S9) } \\
\text { En lien avec la compétence } 3 \text { du domaine personnel : éducation physique et à la santé }\end{array}$} \\
\hline
\end{tabular}


Les excursions (A4 et A8) ainsi que l'expédition (A10) sont des défis exigeants qui demandent des capacités d'endurance cardiovasculaire. Ces ateliers semblent générer chez les jeunes un désir d'améliorer les paramètres facilitateurs tels que la respiration et le positionnement, car un rythme intermittent crée une fatigue précoce (Brannon \& Feist, 2007). Dès la première excursion, les jeunes ont émis des idées à l'égard de leur pratique d'activité physique. Lors de l'atelier 8 (excursion) et de l'expédition (A10), les perceptions recueillies dans le récit du journal de bord de la chercheure-acteure évoquent une modification de l'approche des jeunes concernant la respiration, le dosage d'effort physique, leur positionnement corporel et leur tenue vestimentaire.

De concert avec le cours d'éducation physique et à la santé, la séquence d'éducation par l'aventure en plein air peut favoriser chez le jeune la prise de conscience de ses choix sur son bien-être en matière d'activité physique et faciliter l'intégration de connaissances liées à l'adoption d'un mode de vie sain et actif.

\subsubsection{Bien-être psychologique et habileté à gérer son stress et ses émotions}

À quelques reprises au cours de l'expérimentation, des jeunes ont rapporté avoir vécu des moments de sérénité et ce, principalement lors d'exercices de respiration (A3), d'ateliers de relaxation (A9) ou lors d'activités de confiance (A7). À la lueur des données recueillies, il semble possible que les élèves aient développé progressivement des habiletés à gérer leur stress par des exercices de respiration et 
de relaxation. En fait, les jeunes participants racontent avoir apprécié les activités et exercices de relaxation; toutefois, le constat des intervenants scolaires en rapport avec cette facette est peu éloquent.

Tableau 4.4.3 : Relaxation, gestion du stress, des émotions. Citations (ou écrits) des jeunes A7-Q1 : Au centre du cercle, lorsque tu avais les yeux bandés, comment t'es-tu senti(e)?

$\mathrm{S} 7$ : $\mathrm{J}$ 'imaginais mes amis, je me concentrais beaucoup, je pratiquais ma respiration, je me sentais bien (au milieu d'un cercle d'amis, les yeux bandés)

S15: Molle et j'étais dans ma bulle, je me laissais pousser d'un côté à l'autre.

A9 : Raconte-moi, en tes mots, comment as-tu vécu l'ensemble de l'activité... quelles émotions astu ressenties? Quel(s) moments (s) as-tu préféré(s)?

S3 : J'ai beaucoup aimé la relaxation, car je suis une personne assez stressée [...] la chose qui m'a impressionnée, c'est que nous n'étions pas trop énervés.

S8 : Quand on a fait la relaxation, je me suis senti bien.

$\mathrm{S} 12$ : Pendant la relaxation, je me suis senti très bien et calme.

$\mathrm{S} 22:[\ldots]$ la relaxation, ça nous a fait réfléchir, c'était vraiment plaisant.

S26: [...] nous avons relaxé et cela m'a détendu.

Extraits du journal de bord - Commentaires de la chercheure-acteure et propos des enseignantes lors des entrevues finales

Extraits du journal de bord - A3

Je leur demande de trouver un élément qui peut aider à la concentration; rapidement le sujet 15 me répond : «la respiration ».

J'ai eu conscience qu'ils prenaient soin de respecter les consignes et qu'ils prenaient davantage soin de leur affaire (propos de l'enseignante d'éducation physique et à la santé).

Propos des titulaires de classe en lien avec l'A8 (Entrevue finale)

Parmi les activités significatives, j'ai entendu parler d'une activité avec les yeux fermés [...], sur la respiration aussi [...], le fait de goûter [...] et le mime en duo... mixe de confiance et de sensorialité, où ils devaient se concentrer.

Extrait du journal de bord - A6

J'ai appris que certaines personnes respiraient en surface, mieux vaut respirer avec le ventre, maintenant je le fais avant de me coucher (commentaire de S5 à la chercheure-acteure).

\section{Extrait du journal de bord - A9}

Enseignante 2 a pris soin d'aménager sa classe pour une relaxation avec imagerie mentale. Les jeunes s'installent et je débute par des respirations, suivies d'étirements, de relâchements, de contractions musculaires $[\ldots]$

Je leur demande alors d'imaginer un endroit plaisant.... Enseignante 2 met la musique et me dit de continuer... Fait-il chaud ou froid?...Vente-t-il ou est-ce le clame plat?... Entendez-vous quelque chose? Que faites-vous? Sentez-vous des arômes, des odeurs, de fruits, de pains, de maïs soufflé, d'épices, de sueurs? [...].

Extrait du journal de bord - A10

Les jeunes sont calmes et efficaces. Ils rangent leurs effets personnels. 
Bien que ces sujets n'aient pas été approfondis, il demeure que la séquence d'aventure et d'apprentissage recèle plusieurs moments propices aux échanges, par exemple, autour du feu, lors de moments de collation, de repas ou de temps libre, pouvant servir de tremplin à l'évolution des connaissances sur ces sujets.

\subsubsection{Comportements sains et sécuritaires}

La séquence d'aventure et d'apprentissage a proposé des conditions favorables qui permettent aux jeunes de prendre conscience de l'importance d'entretenir une bonne hygiène de vie, soit au sujet de leur habillement, du sommeil, de l'alimentation ou de comportements préventifs dans les loisirs et les sports.

L'analyse des résultats démontre que c'est l'ensemble de la réalisation de la séquence qui a contribué au déploiement du troisième axe Hygiène et conduite sécuritaire en toutes circonstances. Les propos recueillis au tableau 4.4.4, rapportent l'impact de l'expédition finale (A10) sur cet aspect.

\subsubsection{Hygiène et sécurité}

L'hygiène de vie est un ensemble de mesures destinées à préserver la santé. Avoir une hygiène de vie contribue à son propre bien-être. L'oxygénation du corps (respiration), l'hydratation, l'alimentation, la pratique d'activité physique et le 
sommeil font partie d'une bonne hygiène corporelle, tandis qu'avoir confiance en soi et s'estimer contribuent à préserver son hygiène mentale (Brannon \& Feist, 2007).

À titre d'exemple, des jeunes ont mentionné à quelques reprises, et dans diverses circonstances au cours du projet, être mal ou trop peu habillés, leur prise de conscience a donné lieu à un changement de comportement et ce, pour l'ensemble des participants. L'enseignante d'éducation physique et à la santé considère en matière d'hygiène que les élèves ont effectué un cheminement sur plusieurs plans, dont l'habillement et l'utilisation de l'équipement.

Parmi les exemples explicites, le sujet 11 mentionne le manque de sommeil et le sujet 1 explique plus globalement que pour être en bonne santé, il faut faire des exercices, aller jouer dehors. Le port adéquat du sac à dos fait aussi l'objet de questionnement. Un sac à dos ajusté est essentiel pour maintenir une bonne hygiène corporelle (comportement sécuritaire). L'installation (l'ajustement), la charge (poids) et l'équilibre sont des éléments qui concernent l'utilisation sécuritaire du matériel et de l'équipement. Une prise de conscience à l'égard du port du sac à dos et de la tenue vestimentaire vise l'adoption d'un comportement préventif dans les loisirs et les sports (Martin \& Arcand, 2005). Le tableau 4.4.4 expose l'ensemble des commentaires recueillis sur l'hygiène et la sécurité. 
Tableau 4.4.4: Hygiène et conduite sécuritaire en toutes circonstances

\section{Extraits du journal de bord - Commentaires de la chercheure-acteure et propos des enseignantes lors des entrevues finales}

\section{Citations (ou écrits) des jeunes}

\section{Enseignante 2}

T'sais, ils se sont aperçus que quand t'es mal organisé avec un sac à dos « chambranlant », c'est difficile [...] si j'avais un bon sac à dos [...]

Enseignante d'éducation physique et à la santé L'équipement est important quand tu te déplaces. Ça, les enfants l'ont dit! L'habillement, c'est ressorti aussi. Tu t'habiles pas pour aller en plein air comme tu t'habilles pour aller au centre d'achat... parce que j'ai déjà vu des filles en talons hauts (sortie ponctuelle) [...] c'est vrai, on voyait ça, mais pas dans ce cas-ci.

Extrait du journal de bord - A5

Cet atelier a permis d'effectuer des apprentissages face à la façon de se vêtir et de se comporter dans des situations plus difficiles.

Extrait du journal de bord - A10

[...] Coucher à l'extérieur l'hiver, implique des risques. La première règle d'or, avant de se glisser dans son sac de couchage : porter des vêtements secs [...] Je leur rappelle que l'humidité appelle le froid. [...] vous en avez déjà fait l'expérience. Imaginez ce qui peut arriver si vous couchez à l'extérieur l'hiver avec des vêtements humides! [...] Risque d'hypothermie et même de ne pas vous réveiller. Les jeunes se résignent. Nous rentrons tous à l'intérieur. [...] J'ai une bonne conversation avec Intervenant 1 . Il se sent coupable. Je lui explique qu'il n'a pas à sentir ainsi, il a agi de façon responsable. Pour rejoindre les assises de PFEQ, le plein air doit demeurer sécuritaire. Les jeunes ont à se responsabiliser. Ils n'étaient pas prêts à coucher dehors.
Ce que je vais améliorer à la suite de la réalisation de l'expédition $\mathbf{A 1 0}$

S2 : La prochaine fois... je vais m'habiller mieux, et même si j'ai chaud, je vais enlever des couches.

S7 : M'apporter... plus de linge.

S11 .... pour avoir plus d'énergie, je devais essayer de dormir un peu plus.

S12: La prochaine fois, je vais faire attention à mon bagage, les affaires inutiles, je ne les amènerai pas et mon sac à dos va être équilibré et bien attaché.

S15 : Lorsque je suis partie en expédition, mon sac penchait beaucoup plus vers la droite et j'avais mal à l'épaule droite. La prochaine fois, je vais beaucoup mieux équilibrer mon sac.

S18 : Je devrais installer mon sac mieux que la dernière sortie.

S19 : Pour cette randonnée, j'aurais pu mettre moins de « stock» dans mon sac à dos.

S23 : J'ai eu beaucoup froid aux pieds, alors la prochaine fois je prendrai plus de bas de rechange.

S24 : J'aurais dû apporter une autre paire de bas parce que mes bas étaient « trempes ».

Mettre le poids de mon sac égal de chaque côté et de m'acheter un meilleur sac pour faire du sport.

S25 : Ce serait l'habillement et la charge de mon sac à dos.

S28 : Je vais amener des vêtements de rechange [...]. Aussi je vais mieux attacher mon sac à dos.

Commentaires généraux (retour final)

Pour être en bonne santé, il faut faire des exercices, aller jouer dehors (S1). 
Véhiculée par le concept d'aventure, la notion de risque renforce l'importance d'adopter une conduite sécuritaire. Plus l'activité comporte des risques, plus la sécurité entourant la pratique de l'activité est importante. Ainsi, avant l'expédition finale, une soirée d'information a été tenue pour les parents, deux étudiants au baccalauréat en plein air et tourisme d'aventure de l'Université du Québec à Chicoutimi se sont ajoutés à l'équipe d'intervenants et un plan d'urgence a été mis en place.

En tout temps, l'introduction de la séquence d'aventure et d'apprentissage dans le PFEQ contribue à rejoindre le troisième axe du développement du DGF Santé et bien-être. Bien que certains parents aient ressenti des craintes, d'autres ont souligné la qualité de la planification de la séquence d'aventure et l'encadrement que certains ont qualifié d'exemplaire. Pour favoriser le déploiement de cet axe du DGF Santé et bien-être, nous avons sollicité, à plusieurs reprises au cours de l'expérimentation, la participation des parents et d'acteurs de la communauté (A4-A8-A9-A10).

Au tableau 4.4.5, l'enseignante d'éducation physique et à la santé explique que la séquence d'aventure et d'apprentissage a permis d'intégrer des activités physiques en classe ainsi que dans la famille. Quelques parents rapportent aussi que leur enfant parlait de son expérience à la maison. 
Tableau 4.4.5: Activité physique intégrée en classe, à l'école, dans la famille et dans d'autres milieux

Propos des enseignantes lors des entrevues finales

\section{Enseignante 2}

La séquence d'aventure et d'apprentissage permet de nous rapprocher d'eux [...] de leur montrer que t'es pas juste une enseignante, mais que tu es une personne comme les autres qui possède des compétences et des incompétences, qui a des forces et des faiblesses et qui a des défis à relever.

Enseignante d'éducation physique et à la santé

Ben les parents [...] je repense à la mi-chute. C'était le fun d'les voir. Y'étaient là, pis y'étaient impliqués, pis y'ont ben vu que leurs enfants apprenaient des choses [...]. Ce n'était pas une sortie pour sortir, pis aller se promener sans rien [...] et les parents y'ont embarqué là-dedans à $100 \%$. [...] quand c'est bien présenté, c'est sécuritaire [...] ça va!

Excuse-moi l'expression, mais ce n'était pas « pitcher ». Dans le fond, quand ils sont arrivés là, c'était moins de l'inconnu parce qu'ils avaient couché ici avant (gymnase de l'école). C'est encore (l'idée de) progression, ça se passe bien, hop, là [...] on s'en va dans le bois! [...]. Je ne sais pas s'ils n'avaient pas eu la petite préparation ici [...] comment ça [...] ça aurait peut-être été correct, mais [...]

\section{Commentaires des parents (questionnaire final)}

$\mathrm{S} 1$ : Sensibiliser les jeunes à la sécurité en nature.

$S 2:[\ldots]$ J'espère que d'autres enfants pourront vivre ça [...], et même des plus jeunes seraient tellement contents de faire ça.

S3 : Je trouve bien que les enfants vivent de nouvelles expériences.

S11 : Nous étions certains que tout était bien préparé.

S14 : Il parlait de son expérience à la maison.

S15 : J'avais entièrement confiance aux compétences des organisateurs et aux enseignantes

S18: Il raconte différentes choses qui se passent à l'école ainsi qu'en soirée avec ses amis. Il aime partager et expliquer ses activités.

S24 : [...] c'était bien planifié et sécuritaire. J'espère que ça va continuer parce que j'ai seulement de bonnes critiques de mon enfant.

Les commentaires recueillis portent à croire que l'introduction d'une séquence

d'aventure et d'apprentissage dans l'horaire scolaire régulier favorise un mode de vie

actif et sécuritaire dans la classe, mais aussi dans la famille et dans d'autres milieux, comme l'environnement physique naturel situé à proximité de l'école. 


\subsection{Synthèse des résultats de la réalisation de la séquence d'aventure et les retombées à court et moyen terme}

Afin de mieux mesurer la portée de la séquence d'aventure sur les apprentissages, nous avons à deux reprises, soit à mi-parcours et à la toute fin du projet, demandé aux jeunes si de façon générale le projet Nature et santé leur a permis de découvrir (d'apprendre) de nouvelles choses à l'école. Tant à mi-parcours qu'à la fin du projet, tous les jeunes ont répondu par l'affirmative. Les propos, bien que diversifiés, rejoignent directement plusieurs éléments du PFEQ : la relation de l'homme avec son environnement naturel, la posture, le dosage de l'effort, le fonctionnement du système respiratoire, etc. Ces propos ont été synthétisés et classifiés dans le tableau 4.5.1. 
Tableau 4.5.1 : Synthèse des réponses recueillies à mi-parcours et au retour de l'expédition finale : « Est-ce que le projet Nature et santé leur a permis de découvrir (d'apprendre) de nouvelles choses à l'école ?"

\begin{tabular}{|c|c|c|}
\hline Apprentissages (ou prise de conscience face à) & Retour à mi-parcours & $\begin{array}{l}\text { Retour à la suite de } \\
\text { l'expédition }\end{array}$ \\
\hline $\begin{array}{l}\text { Santé globale (dit avoir senti un impact sur sa } \\
\text { santé) }\end{array}$ & $\mathrm{S} 28-\mathrm{S} 1-\mathrm{S} 18$ & \\
\hline $\begin{array}{l}\text { Alimentation et découverte gustative (bannique, } \\
\text { chocolat noir, clémentines et autres) }\end{array}$ & $\begin{array}{l}\text { S28, S2, S3, S4, S8, S9, } \\
\text { S10, S12, S15, S17, S18, } \\
\text { S19, S20, S21, S22, S23, } \\
\text { S24, S27 }\end{array}$ & $\mathrm{S} 14^{*}, \mathrm{~S} 26$ \\
\hline Hydratation & S11, S16 & S2 \\
\hline Sommeil & & $\mathrm{S} 11, \mathrm{~S} 17, \mathrm{~S} 22, \mathrm{~S} 27$ \\
\hline Habillement & & S2, S7, S23, S24, S26 \\
\hline Respiration & $\begin{array}{l}\text { S28, S6, S8, S9, S12, S15, } \\
\text { S21, S21 }\end{array}$ & S14 \\
\hline Positionnement & $\begin{array}{l}\text { S28, S6, S15, S19 S20, } \\
\text { S21, S23 }\end{array}$ & S14 \\
\hline Effort physique (dosage) & S6, & $\begin{array}{l}\text { S5, S14, S16, S17, } \\
\text { S20, S23, S27 }\end{array}$ \\
\hline Endurance & & S16 \\
\hline Équilibre & $\mathrm{S} 5, \mathrm{~S} 10, \mathrm{~S} 17, \mathrm{~S} 20, \mathrm{~S} 26$ & \\
\hline Souplesse & & $\mathrm{S} 20$ \\
\hline Sécurité (mode) & & $\mathrm{S} 18, \mathrm{~S} 25, \mathrm{~S} 26$ \\
\hline \multicolumn{3}{|l|}{ Compétences transversales } \\
\hline Utilisation des cinq sens & $\mathrm{S} 8, \mathrm{~S} 12, \mathrm{~S} 24^{*}$ & \\
\hline Perception corporelle & S11, S7, S19, S23 & \\
\hline Être en équipe (travailler avec les autres) & S7, S5, S8, S24, S26 & \\
\hline Organisation (méthodologie) & & $\begin{array}{l}\text { S7, S9, S10, S11, S12, } \\
\text { S15, S16, S18, S19, } \\
\text { S21, S22, S23, S26, } \\
\text { S28 }\end{array}$ \\
\hline \multicolumn{3}{|l|}{ Domaines d'apprentissage } \\
\hline Général sur la nature & $\mathrm{S} 1, \mathrm{~S} 18$ & $\mathrm{~S} 3, \mathrm{~S} 8, \mathrm{~S} 5$ \\
\hline Relation entre l'Homme et son environnement & $\mathrm{S} 21$ & S19 \\
\hline Boussole & $\begin{array}{l}\mathrm{S} 6, \mathrm{~S} 10, \mathrm{~S} 12, \mathrm{~S} 17, \mathrm{~S} 20 \\
\mathrm{~S} 21, \mathrm{~S} 22, \mathrm{~S} 24, \mathrm{~S} 25, \mathrm{~S} 26\end{array}$ & \\
\hline $\begin{array}{l}\text { Écosystème (biosphère, abiotique, biotique, } \\
\text { producteur, consommateur, } \\
\text { décomposeur...) }\end{array}$ & $\mathrm{S} 10-\mathrm{S} 15$ & \\
\hline Galaxie (planètes, système solaire) & & $\begin{array}{l}\text { S4, S7, S10, S15, S16, } \\
\text { S18, S20, S23, S24, } \\
\text { S26 }\end{array}$ \\
\hline Reconnaître les traces d'animaux & S3 & \\
\hline Autres & & \\
\hline Faire des nœuds & $\mathrm{S} 5, \mathrm{~S} 17, \mathrm{~S} 21, \mathrm{~S} 23, \mathrm{~S} 25$ & S8 \\
\hline Faire un feu & S24 & S2, S24 \\
\hline Débrouillardise & S9, S24 & S4, S21 \\
\hline
\end{tabular}




\subsection{1 À mi-parcours}

Dans le cadre de la séquence d'aventure, le domaine le plus facile à aborder est sans contredit celui des sciences et technologie, car la nature et l'activation du corps humain offrent de multiples situations d'apprentissage.

En fait, nous avons constaté qu'à mi-parcours, les réponses des jeunes quant à leur apprentissage sont davantage orientées vers la science et la technologie pour l'étude du vivant. Certains, comme les sujets 12 et 15, abordent plus largement la question de la santé (respiration, positionnement, alimentation, endurance physique...), de l'utilisation des cinq sens et de leur découverte gustative : la bannique.

Des principes tels que l'écosystème, la biosphère, le producteur, le consommateur, le décomposeur ont été soulevés par les sujets S10 et S15. Les sujets $17,20,21,12,10,24$ et 25 nous ont mentionné avoir notamment appris à manipuler la boussole.

\subsection{2 À la fin du projet}

Les réponses obtenues sont encore très vastes. Cette fois-ci, nous avons noté que plusieurs élèves relèvent des apprentissages en lien avec le domaine de formation Santé et bien-être et les compétences transversales, principalement celles 
d'ordre personnel et social et d'ordre méthodologique, en ce qui a trait au sens de l'organisation.

Ces réponses nous ont permis de comprendre que l'introduction d'une séquence d'aventure et d'apprentissage recèle un potentiel d'apprentissage beaucoup plus important que nous ne pouvions l'imaginer sur le développement personnel du jeune. L'orientation du projet doit cependant demeurer l'éducation à la santé et le rehaussement de l'estime de soi, même si ceci n'empêche pas que d'autres orientations puissent être mises de l'avant par les titulaires de classe, en lien avec les apprentissages disciplinaires.

\subsubsection{Deux semaines après l'expédition finale, de la séquence d'aventure et d'apprentissage}

Deux semaines après l'expédition finale, nous avons effectué un retour oral avec les jeunes concernant leurs impressions à la suite de la réalisation de la séquence d'aventure et d'apprentissages. Bien que plusieurs jeunes aient encore évoqué des apprentissages théoriques divers, ils ont surtout parlé de confiance en soi (4 sujets), et de connaissance de soi (2 sujets) par, notamment, la capacité à reconnaître ses faiblesses ( 1 sujet). D'autres ont évoqué que l'évolution de la séquence d'aventure a contribué à développer chez eux de la débrouillardise (2 sujets), de la ténacité, de la persévérance et de la maturité ( 2 sujets) (voir tableau 4.5.1). 
Lors du retour -final, la découverte de la nature et son respect ont été mentionnés par au moins deux jeunes. Les aspects de la sécurité en tout temps et du travail d'équipe ont aussi été soulevés. Les jeunes de l'école pilote vivent dans un environnement qui les place fréquemment en contact avec la nature, et la connaissance de cette dernière leur permet d'éprouver un sentiment d'appartenance qui les valorise. Ce type d'atelier semble accroître grandement la motivation des jeunes à l'égard de l'école, ainsi qu'à l'égard des apprentissages liés notamment au corps et à la nature de la science et de la technologie. Il est à noter que le taux d'absentéisme aux ateliers a été minime. Toutefois, pour être évaluées ces questions devront faire partie de recherches ultérieures.

\subsection{La transversalité et la pluridisciplinarité}

Le jumelage des apprentissages disciplinaires aux ateliers d'aventure est primordial à sa réussite, notamment parce que l'éducation par l'aventure offre l'occasion d'établir des relations interprofessorales. À ce sujet, l'enseignante d'éducation physique et à la santé souligne que la réalisation de ce projet lui a permis d'établir une collaboration avec les titulaires de classe de $5^{\mathrm{e}}$ et $6^{\mathrm{e}}$ année et de motiver les jeunes à des apprentissages de techniques moins populaires comme la raquette. Voici son propos :

Bon exemple, je fais une sortie raquette cet hiver [...]. Sans avoir de but la sortie raquette (A8-consolidation du projet intégrateur), ça aurait peut-être été un peu plus difficile... 
surtout pour les motiver. Là en leur disant, on va faire une belle heure de raquette aujourd'hui parce que dans 15 jours, 3 semaines peu importe, vous allez y aller en raquette pour votre projet en classe. Ben t'sais, c'est l'fun. T'as l'objectif au bout! T'sais quand t'as un but à l'autre bout, c'est l'fun. Tu travailles là-dedans, pis c'est motivant, et les élèves aiment plus ça [...]

Ainsi, la mise en place d'une séquence d'aventure et d'apprentissage semble faciliter le travail de l'enseignant d'éducation physique et à la santé, car cette réalisation contextualise les apprentissages et rend les élèves actifs. De plus, elle mentionne que la progression des ateliers semble bien adaptée au rythme physiologique et psychologique des enfants. Selon ces propos, la réalisation de la séquence a contribué à développer les habiletés des élèves pour se maintenir en santé. Voici ses propos :

Tu commences tranquillement pas vite [...] c'était j'pense, même pour les élèves autant [...] mental que physique fallait que ça s'aligne comme ça. [...] les activités étaient toujours ben, ben adaptées. Pis, je te dirais que justement dans cette progression-là [...] ça permettait à tout le monde de vivre le dernier atelier.

Cette opinion est partagée par les titulaires de classe qui sont d'avis que la séquence doit demeurer la même, que le rythme de réalisation et la progression suggérée sont adéquats, que les ateliers sont en interrelation. Selon ces dernières, ce type d'approche enrichit le contenu du curriculum scolaire et encourage le 
décloisonnement des disciplines en mettant en place des situations d'apprentissage authentiques. Voici un survol de leurs opinions :

C'est difficile de couper (la séquence d'aventure et d'apprentissage) [...] c'est un cheminement (Enseignante 1).

Je pense que c'est un projet-année. Si tu coupes, là ça ne sera plus une situation authentique. Une situation authentique, tu l'ouvres, tu la vis et tu refermes. Ce n'est pas juste de vivre une activité [...]. Ça, c'est l'ancien programme. J'pense que ça ne se défait pas. L'atelier vécu avait une progression de 10 ateliers et c'est une situation qui se vit dans une classe réformée (Enseignante 2).

Le projet que j'ai vécu, avec une progression de 10 ateliers, c'est une situation authentique, qui se vit dans une classe, où la réforme est implantée. Une situation authentique t'ouvre, tu vis et tu refermes [...] vivre qu'une activité, ça, c'est l'ancien programme. Ici, nous avons vécu un projet année, c'est difficile de couper, c'est un cheminement [...] c'est dur à juger, mais j'pense que ça se défait pas. (Enseignante 2)

La réalisation de la séquence d'aventure et d'apprentissage est une situation expérientielle dans le contexte approprié sur une période suffisamment longue pour permettre l'intégration des connaissances. La progression des ateliers permet aux élèves de mieux saisir les apprentissages effectués en classe et offre l'occasion de développer leur potentiel et leurs compétences sur les plans physique et relationnel.

Tous les intervenants scolaires semblent d'avis que si l'expérience est répétée, la séquence d'aventure et d'apprentissage, comme elle est construite lors de la 
présente étude doit être conservée, avec de légers ajustements concernant l'arrimage des apprentissages aux ateliers d'aventure et la logistique de certains ateliers.

\subsubsection{La séquence d'éducation par l'aventure : une pédagogie intuitive}

Les titulaires de classe qualifient cette approche de "pédagogie intuitive ». Elles expliquent que le déploiement des compétences disciplinaires demande plus de travail et d'imagination de leur part. Or, elles sont d'accord pour dire que l'introduction d'ateliers d'aventure au PFEQ propose de multiples occasions d'intégrer des apprentissages disciplinaires :

De mon souvenir [...]. Ce que nous avons intégré: les mathématiques, la circonférence [...] avec les recettes [...] toutes les notions de mesures : kilomètre, litre, millilitre... les transferts de grammes, les fractions, les équivalents de fractions [...]. Après ça le français, y'a des lectures [...] y'a eu du vocabulaire [...] y'a eu des projets d'écriture (Enseignante 2).

Ouais, quelques activités de grammaire. Les arts [...] y'a eu des dessins, de la pastel. Art dramatique : improvisation à deux (Enseignante 1).

L'une d'entre elles avance que le potentiel de la séquence d'aventure et d'apprentissage est supérieur à ce qu'elles ont accompli, lors de la présente expérimentation. L'une des titulaires de classe estime qu'elle aurait pu intégrer davantage d'apprentissages disciplinaires; voici son explication : 
Si je le vis dans deux ans, je saurai vers quoi me diriger et je vais être capable de greffer beaucoup plus de disciplines [...] mon vocabulaire, mes lectures, mes recherches seront faites d'avance [...] je serai plus outillée (Enseignante 2).

Les multiples entretiens avec les titulaires de classe démontrent la place de l'intégration des domaines d'apprentissage dans la séquence d'aventure. ̇̀ titre d'exemple, le domaine des langues s'enseigne par des lectures de textes préparatoires et préalables aux sorties, par la rédaction de textes variés en lien avec les ateliers vécus et par l'évaluation de la communication orale des jeunes en cours d'atelier en plein air.

Le domaine de la mathématique et de la science et de la technologie est exploré au cours des observations en nature, d'expérimentations concrètes en atelier, d'utilisation d'instruments scientifiques et lors de la réalisation du projet intégrateur, et aussi, lors de la réalisation de recettes culinaires. C'est notamment ce que soulève l'enseignante 1 :

(La réalisation de recettes) Tu travailles les fractions, les pourcentages, les rapports [...] et y'a un but! Les mises en situation étaient bonnes et partaient du vécu de l'enfant, c'était du concret pour l'enfant.

Par la réalisation de projets en classe, les jeunes sont invités à utiliser leurs connaissances en établissant des liens entre leur vécu et la théorie. Bien qu'il soit 
difficile d'évaluer si les jeunes participants ont réalisé des transferts de connaissances, ils ont vu, touché, senti des choses, ils ont cherché des informations (livres, Internet...), et ils ont effectué des liens avec leur vécu :

Ils vont passer des commentaires liés à ce qu'ils ont appris en plein air. T'sais, ils vont faire des liens avec leur vie de tous les jours [...] comme avec une nouvelle connaissance, ils vont essayer de la rattacher à leur vécu [...] (Enseignante 2).

Bien que l'introduction de notions disciplinaires au cœur de la séquence d'aventure et d'apprentissage soit possible, il semble essentiel que :

a) Les apprentissages s'effectuent dans un contexte de gestion de la classe qui respecte les principes du renouveau pédagogique et ce, malgré environnement physique naturel. L'élève doit connaître les intentions éducatives de l'atelier et se sentir responsabilisé par une tâche ou un rôle qu'on lui a confiés.

b) Le personnel scolaire impliqué dans la réalisation de la séquence d'aventure et d'apprentissage connaît et approuve dès le début de l'année scolaire l'ordonnancement et le contenu des ateliers constituant la séquence.

c) Les titulaires de classe impliqués disposent d'un certain temps pour s'approprier les intentions éducatives de la séquence, afin de construire 
des - situations d'apprentissage et d'évaluation (SAE) réalisables dans ce contexte.

d) Le leadership partagé selon les compétences des intervenants scolaires, par exemple le titulaire de classe, l'enseignante d'éducation physique et à la santé et le coordonateur-intervenant, etc., et le respect mutuel des rôles d'autorité entre les intervenants en relation avec les élèves.

Si ces conditions sont respectées, la mise en place d'une séquence d'éducation par l'aventure propose d'innombrables possibilités d'apprentissage, notamment dans les champs disciplinaires du français, de la mathématique, de la science et de la technologie, de la géographie et surtout de l'éducation physique et à la santé.

\subsection{Les commentaires généraux des différents adultes impliqués à la suite de réalisation de la séquence}

Dans l'espoir de saisir le potentiel de ce type d'intervention éducative en matière de santé (Savoie-Zajc, 2001) et de qualifier le processus de transformation des pratiques, les enseignantes, la direction et les parents ont été questionnés au sujet de leur perception par rapport à l'expérience. La majorité des commentaires semble converger dans le même sens : l'expérience doit se répéter pour favoriser l'épanouissement des jeunes en milieu scolaire et intégrer les principes de l'éducation relative à la santé! 


\subsubsection{La direction de l'école}

Selon la direction de l'école, le projet cadre avec les visées du projet éducatif et génère de multiples effets positifs. La direction est assurée que les titulaires de classe ont mis à l'essai des méthodes d'enseignement et des stratégies d'apprentissage différentes faisant référence aux ateliers vécus : observations et expérimentation communes pour le maître et des élèves en nature et pluridisciplinarité. Il considère également avoir perçu un changement d'attitude chez des élèves quant à leur motivation à l'égard de l'école.

Relativement à l'implantation d'un tel projet à la grandeur de l'école, les propos de la direction de l'école sont sans équivoque : "L'implication de tout le personnel de l'école est essentielle ». Pour qu'un tel projet soit bien perçu dans le milieu scolaire, sa portée éducative doit absolument être présentée à tous les intervenants scolaires. Ainsi, il est essentiel d'accorder un temps de concertation avec le coordonnateur et les intervenants du milieu afin de mobiliser tout le personnel de l'école.

\subsubsection{Les parents}

La mise en place de la séquence d'aventure n'aurait pas été possible sans l'approbation de la direction et le soutien des parents. Or, malgré les deux rencontres formelles d'information, certains parents éprouvaient toujours des craintes envers la 
pertinence du projet en milieu scolaire. Toutefois, lors de l'atelier 9 (présentation par les jeunes du projet intégrateur à la communauté), plusieurs parents ont ouvertement appuyé la démarche. Voici quelques commentaires en ce sens :

Je croyais que lorsque les jeunes allaient dehors, ils n'apprenaient rien, mais je constate que c'est tout autrement.

Je sens que mon enfant se réalise dans ce type d'activités. Il m'en parle beaucoup à la maison.

Nous sommes intéressés par les apprentissages effectués particulièrement en ce qui a trait à son bien-être.

Ainsi, selon la perception des parents la séquence d'aventure et d'apprentissage propose de nombreuses situations qui renforcent les compétences des jeunes, tant sur le plan personnel que scolaire.

Les parents qui ont assisté à des sorties antérieures mentionnent la pertinence et l'émerveillement des jeunes, sans compter les multiples apprentissages effectués, qui se rendent jusqu'à la maison. Plusieurs parents étaient heureux de constater que leur enfant apprenait lors des sorties (Extrait du journal de bord de la chercheure-acteure - A9)

Ce cheminement éducatif novateur et sécuritaire s'effectue dans le respect de la nature et des autres. La séquence d'éducation par l'aventure conduit à de multiples apprentissages et développe le potentiel des jeunes participants. Croire en soi, apprendre à travailler en équipe, avoir une meilleure connaissance de soi, être capable de reconnaître ses faiblesses et voir son corps comme une petite machine 
amusante remplie de compétences, d'habiletés et de potentiel, voilà quelques éléments que les jeunes ont retenu du projet d'aventure éducative en santé au cœur du PFEQ : Nature et santé. 


\section{CHAPITRE 5}

\section{Discussion}

La discussion est construite à partir de l'analyse et de l'interprétation des résultats de l'expérimentation de la séquence d'aventure. L'objectif principal de cette expérimentation qui s'est déroulé avec 29 élèves du troisième cycle du primaire, sur une période de 7 mois, consistait à vérifier les effets de l'introduction d'ateliers d'éducation par l'aventure en plein air dans le programme de formation de l'école québécoise.

L'analyse des résultats démontre que la séquence d'aventure et d'apprentissage comme elle a été vécue dans la présente étude :

- Soulève une conscientisation en matière de santé;

- produit des retombées potentiellement positives sur l'ensemble des facteurs de développement individuel du jeune (annexe 1), dont l'estime de soi;

- enrichit certains apprentissages ciblés pour le troisième cycle du primaire. 
La mise en place d'une telle séquence en milieu scolaire exige une structure particulière qui respecte une progression spécifique (Bilodeau, 2005a; Joplin, 2008) et adopte une approche d'autonomisation (Parcel et al., 2000). Martin et Arcand (2005) estiment à ce sujet que pour mettre en valeur des actions de promotion et de prévention auprès des jeunes, les acteurs et concepteurs du projet doivent avoir une vision globale et systémique des actions, et proposer un projet qui rejoigne l'intérêt des jeunes, tout en répondant à leurs besoins. Sans ces éléments, Priest et Gass (1997), ainsi que Neill et Richard (1998) affirment que les objectifs visant notamment le rehaussement de l'estime de soi pourraient être compromis.

L'analyse des résultats présente les effets manifestés sur le développement du jeune sur une base individuelle (annexe 1), soit le rehaussement de l'estime de soi, le développement de compétence sociale, l'adoption de saines habitudes de vie et de comportements sains et sécuritaires en matière de Santé et bien-être. Les opinions des intervenants scolaires et de la chercheure corroborent les écrits de Neill et Richard (1998) concernant le lien entre les éléments émergents à la suite de l'expérimentation et les modèles expérimentaux d'aventure éducative en plein air (tableau 2.4.1).

La littérature consultée sur l'apprentissage expérientiel (Joplin, 2008; Rabiola \& O'Keefe, 1999) suggère que l'introduction d'ateliers d'éducation en plein air améliore la perception des élèves face à eux-mêmes et leur permette de découvrir leur potentiel. Aussi le dépassement de soi exigé par les différents ateliers d'aventure 
contribue à-1'émergence d'un sentiment de fierté et de satisfaction personnelle (Duclos, 2000; Reasoner, 1982).

Les prochaines sections abordent les avenues potentielles à l'introduction d'ateliers d'éducation par l'aventure dans le programme de formation de l'école québécoise. La lecture du PFEQ (2001) ainsi que l'analyse des données recueillies au cours de la présente étude ont dressé plusieurs constats sur la place d'une telle séquence dans le système scolaire québécois. Ils ont donné naissance à un modèle descriptif (schémas 5.1.1) expliquant la vision systémique de l'introduction d'une séquence d'aventure sur le PFEQ.

Ensuite, la réflexion s'étend à l'impact des ateliers vécus selon les modèles théoriques d'éducation à la santé, et sur les facteurs clés du développement du jeune en matière de Santé et bien-être. Finalement, pour favoriser l'évolution des défis en matière de santé en milieu scolaire, nous proposons un modèle d'implantation de la séquence d'aventure en milieu scolaire.

\subsection{Une vision systémique de la séquence d'ateliers}

La littérature (Martin \& Arcand, 2005; Motta, 1998; Mérini et al., 2004; Turcotte, 2006) rapporte que l'introduction d'ateliers d'éducation à la santé doit s'effectuer en complémentarité avec un projet réalisé en classe. En ce qui concerne la présente étude, les ateliers réalisés s'inscrivent dans le plan de réussite du projet 
éducatif de l'école. Le jumelage des orientations de la séquence aventure à une séquence d'apprentissage a servi à multiplier le nombre de situations propices à l'accomplissement de tâches intégratrices ou d'activités d'apprentissage spécifiques, en plus de satisfaire les élèves (Dugual, 1999; Lapointe et al., 2000; Famose, 2001; Godin, 2006).

À la suite de l'analyse des résultats et en accord avec les écrits de Neill (2000) et Gass (1999), la séquence d'aventure élaborée et réalisée pour la présente étude se révèle un instrument doté d'un grand potentiel pour le développement et la consolidation de compétences sociales et de compétences disciplinaires.

Le schéma 5.1.1, réalisé par la chercheure, illustre la place de l'éducation par l'aventure dans le PFEQ (PFEQ, 2001). L'introduction d'une séquence d'aventure se juxtapose à un projet de classe et transcende les assises du PFEQ. 


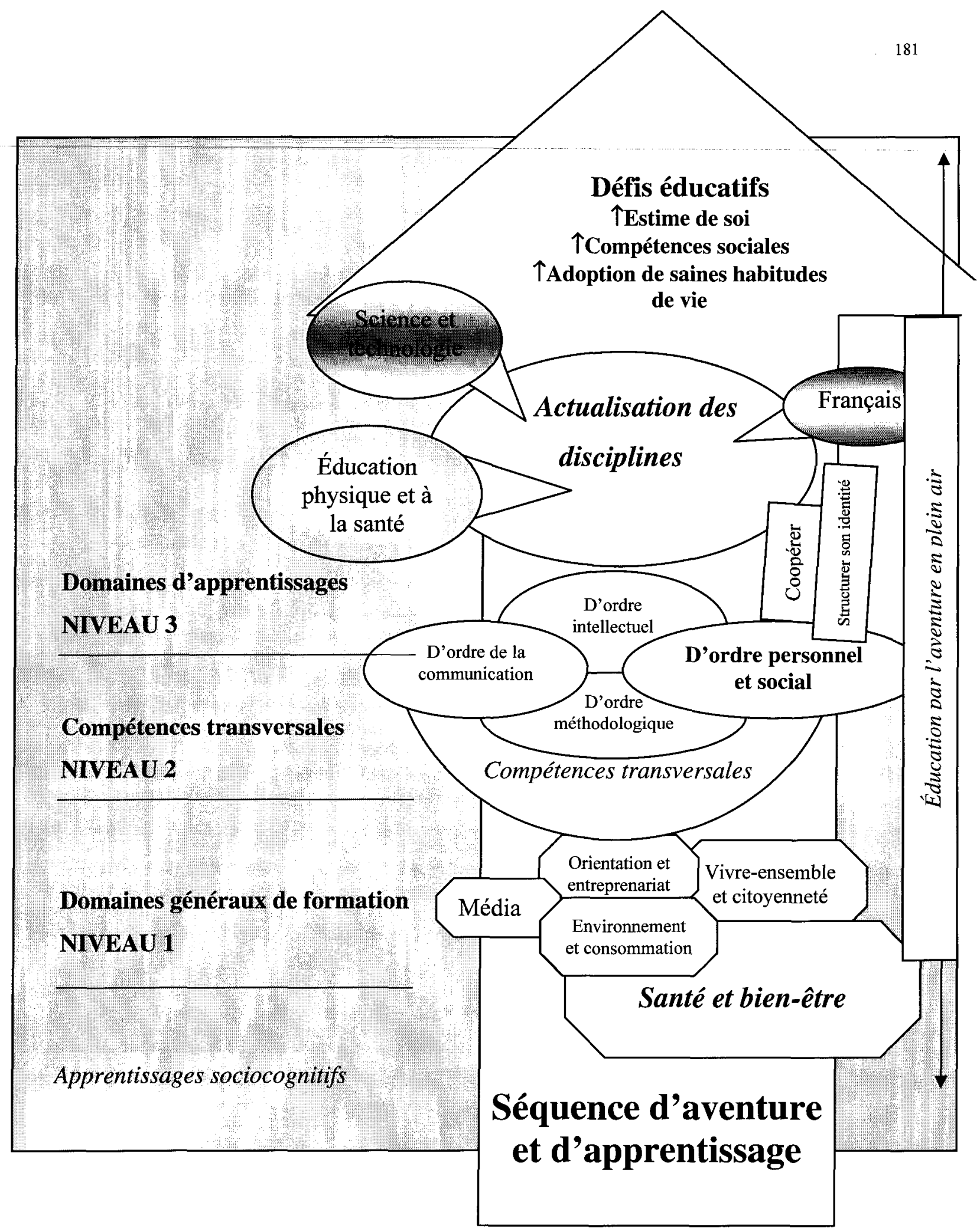

Schéma 5.1.1 : Modèle descriptif de l'éducation par l'aventure et le plein air dans le PFEQ 
La lecture du schéma descriptif, ci-dessus, s'effectue de bas en haut suivant la flèche intitulée "Séquence d'aventure et d'apprentissage ». Le premier niveau du schéma 5.1.1. illustre que l'éducation par l'aventure prend ancrage sur le DGF Santé et bien-être et propose une approche fondant le développement des dynamiques individuelles sur l'expérience sociale, c'est-à-dire le sociocognitif (Vygotski, 1985; Garnier et al., 1991; Bowen et al. 2001, tiré de Martin \& Arcand, 2005) Le deuxième niveau du même schéma souligne l'émergence de compétences transversales d'ordre personnel et social, comme il a été démontré lors de l'analyse de résultats, et aussi invite au déploiement d'autres compétences de ce niveau. Le troisième niveau du schéma expose les domaines d'apprentissage rejoints ou abordés, lors des ateliers d'éducation par l'aventure, vécus par les jeunes du troisième cycle du primaire. Enfin, le haut de la flèche présente les éléments émergents de la séquence d'aventure et d'apprentissage soit le développement des saines habitudes de vie, les compétences sociales et le rehaussement de l'estime de soi.

L'analyse de contenu montre que la réalisation de la séquence d'aventure et d'apprentissage en milieu scolaire présente de nombreux défis éducatifs et ce, tant pour les intervenants scolaires (enseigner dans un nouvel environnement en utilisant d'autres stratégies pédagogiques) que pour les jeunes vivant l'aventure (accomplir des efforts physiques soutenus, s'adapter à de nombreuses situations nouvelles). Par exemple, les ateliers d'éducation par l'aventure proposent des expériences qui comportent des éléments d'excitation, d'incertitude, d'effort, de risques (réels ou 
perȩus) et ce, pour l'ensemble des participants (Priest \& Gass, 1997). Aussi, dans le but d'instruire, de socialiser et de qualifier en tenant compte du principe de l'égalité des chances pour tous (PFEQ, 2001), ce mode d'intervention privilégie l'élaboration conjointe de situations d'apprentissage (titulaires de classe et enseignante d'éducation physique et à la santé) et l'utilisation de l'environnement naturel.

Également, le soutien et la collaboration des titulaires de classe, de la direction de l'école, des parents et de la communauté ont grandement contribué à la réalisation et à l'harmonisation de la séquence d'aventure avec les assises du PFEQ (2001). Ainsi, l'analyse des résultats démontre que les situations d'apprentissage produites par la réalisation des ateliers ont contribué au développement de multiples compétences chez les jeunes participants ainsi qu'à l'acquisition de nouvelles connaissances, particulièrement dans les disciplines suivantes : français, science et technologie et éducation physique et à la santé. 


\subsection{Les portées pédagogiques de la séquence d'aventure}

Martin et Arcand (2005) et Martinot (2001) affirment que la réussite éducative repose sur l'émancipation du jeune. La séquence d'aventure et d'apprentissage, selon l'analyse de résultats, aurait le potentiel de :

a) Rallier l'équipe-école autour d'un même projet.

b) Favoriser les apprentissages pluridisciplinaires en milieu naturel.

c) Renouveler certaines pratiques pédagogiques.

Le titulaire de classe et l'enseignant en éducation physique ont effectué une mise en commun des contenus théoriques qui sont susceptibles d'émerger en cours d'ateliers. Chacun a accepté d'inclure, lors de la réalisation des ateliers, une partie de la responsabilité éducative de l'autre. Selon Lasnier (2000), toutes les stratégies pédagogiques pertinentes se composent de situations d'apprentissage contextualisées et complexes qui favorisent le décloisonnement des disciplines. La séquence d'aventure et d'apprentissage représente, par définition, une stratégie pédagogique novatrice.

Plusieurs auteurs rappellent qu'une vision globale de la santé et de la réussite va au-delà de la simple superposition d'activités (Martin \& Arcand, 2005; Turcotte, 2006). Donner un sens aux apprentissages s'effectue notamment par le développement de compétences multiples (savoir agir) (Lasnier, 2000) autour d'un 
même projet. La séquence d'aventure est une progression d'ateliers qui mène au développement de plusieurs compétences transversales et disciplinaires (annexe 5) et aussi au rehaussement de l'estime de soi et l'acquisition de saines habitudes de vie.

Le tableau 5.2.1 présente les compétences perçues comme émergentes et utilise un système de codification étoilé pour démontrer l'impact de chaque atelier sur leur déploiement. La présence de trois étoiles signifie l'émergence majeure de cette compétence et une étoile signifie un déploiement modeste de la compétence. À titre explicatif, les ateliers 1, 3 et 6 demandent aux jeunes d'utiliser leurs sens pour accomplir certains défis, de s'ouvrir aux stimulations environnantes ou de mettre à profit leurs ressources personnelles, deux éléments qui constituent la compétence transversale 7, Structurer son identité. Les ateliers 2, 5, 7 et 9 travaillent davantage sur le développement de la coopération (compétence transversale 8), portant sur l'entraide, le respect mutuel et le respect de l'environnement. Les deux excursions (ateliers 4 et 8 ) et 1'expédition finale (atelier 10) exigent un effort cardiovasculaire supérieur. Ces ateliers contribuent donc à consolider les apprentissages et à accroître la conscientisation des jeunes par rapport aux conséquences de leurs choix personnels sur leur santé et leur bien-être (DGF Santé et bien-être, axe 2). Le développement de l'ensemble de ces compétences vise à accroître une conscience de soi, par la perception des signaux corporels, favorisant ainsi le bien-être physique et psychologique des individus (Martinot, 2001) et l'émergence de l'estime de soi positive (Reasoner, 1982). 
Tableau 5.2.1 : Éléments émergents des ateliers d'aventure et de plein air, réalisés par les jeunes du troisième cycle du primaire $\left(5^{\mathrm{e}}\right.$ et $6^{\mathrm{e}}$ année) École Fréchette de 1'Anse-Saint-Jean.

\begin{tabular}{|c|c|c|c|c|}
\hline & 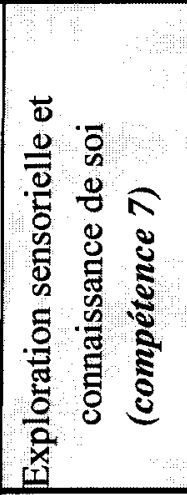 & 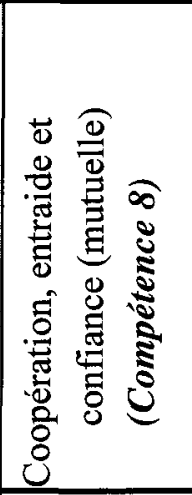 & 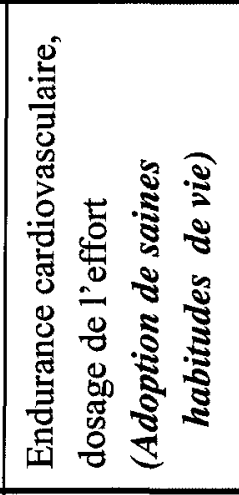 & $\begin{array}{l}\text { ÉLÉMENTS } \\
\text { ÉMERGENTS DANS } \\
\text { CHAQUE ATELIER } \\
\text { DE LA SÉQUENCE } \\
\text { D'AVENTURE }\end{array}$ \\
\hline $\begin{array}{l}\text { A1 - Trésor à la } \\
\text { boussole }\end{array}$ & *** & ** & * & Structurer son identité \\
\hline $\begin{array}{l}\text { A2 - Randonnée et } \\
\text { jeux coopératifs }\end{array}$ & * & *** & * & Coopérer \\
\hline $\begin{array}{c}\text { A3 - Jeux de } \\
\text { sensibilité } \\
\text { corporelle }\end{array}$ & $* * *$ & * & ** & Structurer son identité \\
\hline $\begin{array}{l}\text { A4 - Randonnée à la } \\
\text { chute }\end{array}$ & * & ** & *** & $\begin{array}{c}\text { Adoption de saines } \\
\text { habitudes de vie }\end{array}$ \\
\hline A5-Défis coopératifs & $*$ & $* * *$ & $* *$ & Coopérer \\
\hline $\begin{array}{l}\text { A6- Défis et } \\
\text { dégustation }\end{array}$ & **** & * & ** & Structurer son identité \\
\hline $\begin{array}{l}\text { A7- Bouffe et } \\
\text { confiance }\end{array}$ & *** & $* * *$ & * & Coopérer \\
\hline $\begin{array}{l}\text { A8 - Randonnée } \\
\text { raquette }\end{array}$ & ** & $*$ & $* * *$ & $\begin{array}{l}\text { Adoption de saines } \\
\text { habitudes de vie }\end{array}$ \\
\hline $\begin{array}{l}\text { A9- Coucher } \\
\text { intérieur, bouffe et } \\
\text { présentation }\end{array}$ & * & $* * *$ & ** & Coopérer \\
\hline $\begin{array}{c}\text { A10 - Le jour J : « on } \\
\text { couche dehors! » }\end{array}$ & ** & * & *** & $\begin{array}{l}\text { Adoption de saines } \\
\text { habitudes de vie }\end{array}$ \\
\hline
\end{tabular}




\subsection{Un modèle d'éducation à la santé}

À ce jour, plusieurs théories en matière d'éducation à la santé s'entendent pour dire qu'une attitude gagnante en matière de santé se développe, par les relations sociales, le renforcement de compétences personnelles (Martin \& Arcand, 2005; Turcotte, 2006) et l'émergence de sentiments positifs à l'égard des expériences vécues. La séquence d'aventure offre une suite d'ateliers qui modifient le contexte environnemental conduisant vers une modification des comportements.

La figure 5.3.1 est en quelque sorte un des pivots centraux servant à la compréhension de l'impact potentiel d'un tel mode d'intervention en matière d'éducation à la santé. L'ensemble des constats émergents retenus dans le présent mémoire y est présenté. Exposant une vision holistique des différents modèles et théories de l'éducation à la santé privilégiés en Amérique du Nord, la figure 5.3.1 reprend fidèlement la figure 2.1.2, et introduit six encadrés qui précisent l'ensemble des éléments, selon les variables ou facteurs ciblés par la présente recherche. Par exemple, la séquence d'aventure et d'apprentissage (variable externe, encadré 1) influence les comportements des jeunes en améliorant leurs attitudes sur les plans cognitifs (encadré 2) et affectifs (encadré 3), ce qui modifie les normes qu'ils perçoivent et le contrôle qu'ils peuvent exercer sur leurs comportements (encadré 5). Quant à l'encadré 6, il énumère les ressources et facteurs qualifiés de facilitants, car ceux-ci ont la capacité d'influencer les jeunes à adopter des comportements sains. 


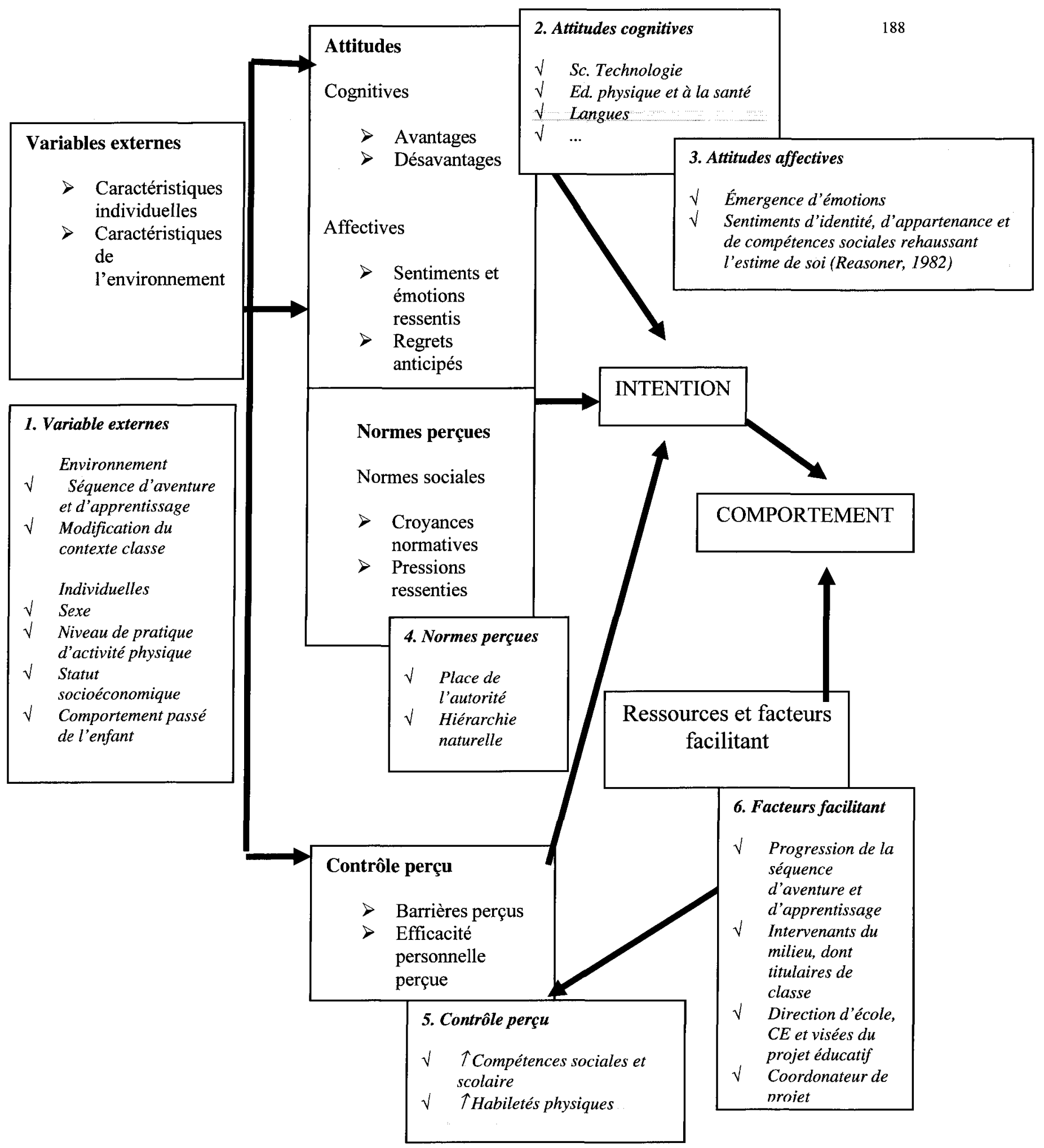

Figure 5.3.1 : Modèle intégrateur de Godin (2002) en lien avec les différents modèles et théories de l'éducation à la santé privilégiés en Amérique du Nord (Turcotte 2006) adapté selon les résultats d'analyse de la présente étude. 
La figure 5.3.1 situe donc les différents constats soulevés dans l'analyse des résultats. Ces facteurs exercent une influence sur la motivation des jeunes participants à adopter un changement de comportement en matière de santé (Godin, 2006). Le contenu de cette figure (figure 5.3.1) sert d'assise à toute la section 5.3 de la discussion du présent mémoire.

\subsubsection{Variables externes}

La séquence d'aventure et d'apprentissage engendre des modifications sur le plan de la gestion du groupe-classe (encadré 1), un remaniement du contexte «classe » s'impose auprès des élèves. L'introduction des ateliers d'aventure au curriculum entraîne une série de répercussions, notamment à l'égard des normes de comportement à adopter et des croyances dans les rôles sociaux normatifs. La modification de repères déstabilise le jeune sur le plan environnemental et sur le plan social, transformant ainsi la dynamique de groupe. En effet, la littérature rapporte que l'aventure provoque des situations relationnelles multiples avec les compagnons de route et avec nous-mêmes (Bilodeau, 2001; Cheryl \& Lupton, 1999) favorisant ainsi l'émergence de compétences sociales (Martin \& Arcand, 2005), d'où la présence plus fréquente d'échanges entre les élèves vers la fin de la séquence.

Par ailleurs, la catégorisation des résultats et l'analyse de contenu (Bardin, 2007) ont montré que la modification du contexte-classe a contribué à soutenir 
l'intérêt des jeunes pour le milieu scolaire. Par exemple, à la suite de l'expérimentation les titulaires de classe ont affirmé avoir perçu un accroissement de la motivation des élèves envers l'école particulièrement dans la réalisation de tâches, en lien avec le projet, par exemple, la recherche d'informations sur Internet, la conception de maquette ou d'affiche. Aussi, plusieurs jeunes participants affirment avoir partagé l'évolution de la recherche d'informations avec leurs parents et avoir ressenti de la satisfaction tout au long de la réalisation de la séquence d'aventure et d'apprentissage, en découvrant notamment que l'école peut leur offrir davantage. Il s'agit de l'exploration de nouvelles techniques, de connaissance de nouveaux aliments, d'exploration du milieu naturel, etc.

\subsubsection{Dimension cognitive}

La progression des ateliers offre plusieurs occasions d'effectuer des apprentissages contextualisés. L'apport de la dimension cognitive dans l'enseignement en éducation à la santé doit inclure un processus d'analyse des conditions personnelles, sociales et environnementales concernant les habitudes de vie et de santé (Turcotte, 2006; Tones \& Green, 2004). L'expérimentation vécue encourage l'acquisition d'attitudes cognitives étroitement en lien avec le domaine des langues, de la science et de la technologie et le domaine de l'éducation physique et à la santé (encadré 2, figure 5.3.1). Les connaissances disciplinaires acquises par les jeunes en classe ou lors des ateliers d'aventure contribuent à identifier les avantages et désavantages de leurs actions, principalement en ce qui a trait à la 
condition physique, à la tenue vestimentaire, à l'alimentation et à la relaxation. Induite par un processus d'apprentissage expérientiel (Joplin, 2008; Dewey, 1939 tiré de Rabiola \& O'Keefe, 1999), l'évolution des connaissances s'est manifestée notamment à l'étape de rétroaction (Joplin, 2008) qui suit chaque atelier. L'observation de phénomènes naturels ou physiologiques conduit les jeunes vers une compréhension de leurs besoins fondamentaux et des conséquences de leurs choix personnels sur leur santé et leur bien-être. Les jeunes participants ont édifié des liens au cours de la réalisation de la séquence entre la sensation perçue et le comportement à adopter pour mieux répondre à leur bien-être.

\subsubsection{L'environnement naturel et son impact sur l'estime des jeunes}

L'environnement naturel, riche en stimulations, expose les élèves à de nombreux questionnements laissant l'occasion aux titulaires de classe de les guider dans la recherche de réponses. Inscrits comme des savoirs essentiels ciblés au troisième cycle du primaire, l'univers vivant, de la Terre, de l'espace et de l'interaction entre l'humain et son milieu viennent, par leur étude d'enrichir le contenu de la séquence d'aventure. Le PFEQ (2001) spécifie que, pour être à l'aise dans son milieu et éprouver un sentiment de valorisation et d'appartenance à l'égard de celui-ci, il est nécessaire d'y vivre, de le connaître et de se connaître.

Inscrit comme le premier apprentissage commun du domaine $\mathrm{du}$

développement personnel, l'estime de soi correspond à la valeur que l'individu 
s'accorde (Martinot, 2001) et constitue un facteur éthique et d'efficacité recherché en matière de santé et de réussite scolaire. Turcotte (2006), Martinot (2001), Duclos (2000) et Arcand et al. (1998) estiment que des interventions efficaces en éducation à la santé dégagent un sentiment de confiance, d'appartenance et de réussite.

L'analyse démontre que la réalisation de ce projet a généré chez les jeunes une gamme d'émotions (encadré 3, figure 5.3.1) qui a contribué à rehausser l'estime de soi des jeunes (Reasoner, 1982). Gass $(1993,1999)$ explique que le principe d'aventure concerne davantage l'aspect affectif (personnel ou social) et physiologique de l'attitude. Ainsi, la progression de la séquence propose le développement des zones de confort humain et de confort environnemental qui encourage la prise de conscience du jeune avec la nature et avec lui-même. Ce phénomène a des impacts positifs sur la santé des participants (Ann Klint, 1999) et les conduit vers un désir d'accomplissement personnel. Neill et Richard (1998) et Hattie et al. (1997) évoquent que les efforts physique et mental exigés dans les activités d'aventure ramènent l'individu vers une conscience de lui-même qui renforce son estime et son efficacité personnelles et ce, plus que tout autre programme d'éducation. Ainsi, l'introduction de la séquence d'aventure et d'apprentissage contribue à améliorer le rapport des jeunes avec leur identité personnelle, développe un sentiment d'appartenance au groupe et favorise l'émergence de sentiments positifs tels que la satisfaction personnelle (tableau 4.2.1). 
L'expression chez les élèves de la fierté relative à leurs réalisations et leur implication physique à l'effort façonne le développement des compétences sociale et scolaire des participants (encadré 5, figure 5.3.1), qui progressivement, sous l'angle affectif, contribuent à solidifier leur estime de soi (encadré 3, figure 5.3.1).

\subsection{Compétences sociales et habiletés physiques}

La plupart des ateliers de la séquence demandent de la force, de la souplesse, de l'endurance physique, de la coordination et de l'équilibre, dimension du soi physique (Famose, 2001). Les résultats de nos analyses démontrent que la séquence d'aventure modifie la perception des jeunes envers leur efficacité personnelle (encadré 4, figure, 5.3.1) dans la pratique d'activité physique. Les ateliers de confiance, de communication et de résolution de problèmes exigent pour la majorité des participants une adaptation qui met l'accent sur la dimension relationnelle et contribue graduellement à générer une transformation du savoir social (Vygotski, 1985). La réalisation des ateliers d'aventure demande aux jeunes de se dépasser sous plusieurs dimensions de leur être, tout en cultivant leur compétence sociale.

Par définition, l'aventure comporte des risques qui génèrent des situations précaires (Priest \& Gass, 1997). À titre d'exemple, d'octobre à avril, les élèves ont eu à affronter des conditions climatiques variables : la pluie a été au rendez-vous à l'atelier 4; la température a été glaciale $\left(-25^{\circ} \mathrm{C}\right.$, avec le facteur vent) lors de l'atelier 
5 et le vent a été de la partie lors de 1'expédition finale. Plusieurs participants ont surmonté des épreuves personnelles d'ordre physique ou relationnel et l'analyse des résultats indique que la progression de la séquence d'aventure contribue à l'éclosion de sentiments d'appartenance au groupe et d'autoefficacité (Martinot, 2001).

\subsubsection{Relations des élèves avec les différents intervenants impliqués}

Tout comme les élèves, les enseignants participent aux excursions et à l'expédition. L'analyse des résultats montre que ce type d'approche redéfinit la relation enseignant-élèves. Les observations démontrent une transformation de l'image de l'autorité, perçue comme plus authentique et humaine (encadré 4, figure 5.3.1). Selon Bilodeau (2005b), l'un des plus grands bienfaits de l'éducation par l'aventure serait l'évolution de la relation de confiance entre le jeune et les intervenants et entre les jeunes eux-mêmes. Accompagner les jeunes dans leurs apprentissages et vivre avec eux des situations concrètes modifieraient, selon l'analyse de contenu effectué, les perceptions des jeunes et des intervenants scolaires par rapport à eux-mêmes et à 1'égard des pairs et de l'autorité. La participation des titulaires des classes aux ateliers bonifierait alors l'image de l'autorité, en plus d'intervenir sur la hiérarchie qu'on retrouve habituellement en classe. Leur confiance à l'égard du leadership de la chercheure-acteure a contribué à transformer la perception relativement aux relations interpersonnelles des jeunes et à encourager la mise en place d'un climat de confiance. Ainsi, en modifiant le contexte en classe, le 
déploiement des habiletés-de communication et-de coopération opère différemment et métamorphose les dimensions relationnelles des participants.

\subsubsection{La relation avec les pairs}

Les défis, reliés à l'aventure en plein air, vécus lors des ateliers préparatoires, les excursions et l'expédition, exigent à différents niveaux le dépassement personnel des participants et développent les habiletés de communication, de résolution de problèmes, de gestion du stress et de prise de décisions (Neill \& Richard, 1998; Arcand et al., 1998). La totalité des ateliers fait appel à la coopération. La réalisation de la séquence d'aventure et d'apprentissage engendre de multiples interactions sociales qui aident le jeune à découvrir ce qui influence son comportement (Tones \& Green, 2004) et, progressivement, contribue à développer ses capacités d'expression, de réflexion et d'analyse (Sandrin-Berthon, 1997).

Bien que la littérature mentionne une légère diminution de la structure du soi social entre 8 et 12 ans (Henry, 1996), les manifestations de coopération et d'entraide sont abondantes et ce, tout au long de l'expérimentation. Les jeunes rapportent une considération grandissante envers leurs pairs, sans égard au sexe de l'individu. L'enseignante d'éducation physique et à la santé signale avoir remarqué, au cours de l'année scolaire, une homogénéisation progressive de ce groupe, reflétant l'acceptation de tout un chacun avec leurs forces et leurs faiblesses. Dans le 
même ordre d'idées, nos analyses soulignent le développement de la compétence Coopérer (CT. 8) et l'amélioration de la collaboration au sein du groupe, en classe et lors des ateliers d'aventure éducative. La progression des ateliers a favorisé la manifestation d'un gain d'autonomie, un éveil de conscience (awareness ${ }^{12}$ ) et surtout une meilleure collaboration entre les participants. Dans ce nouveau contexte, les élèves découvrent progressivement leur rôle au sein du groupe-classe.

\subsection{L'impact des ateliers sur les facteurs clés du développement du jeune en matière de Santé et bien-être}

L'éveil sensoriel que propose la séquence d'aventure sous forme d'ateliers d'acclimatation semble, selon De Koninck (2004), généralement oublié dans l'éducation de nos enfants. Or, la connaissance de soi est une dimension centrale du développement psychique (Brassard \& Mongeau, 2004) et passe principalement par l'ouverture aux stimulations environnantes par les sens.

Le modèle de santé par l'action (HAM - figure 2.1.3) propose, pour modifier les actions des individus en matière de santé, de travailler sur trois plans: l'environnement, les habiletés et les connaissances. Les caractéristiques de l'environnement étant déjà modifiées par l'introduction des ateliers en plein air,

12 La langue anglaise utilise le terme awareness pour expliquer une conscience sensorielle toujours plus éveillée. Neill et al. (1998) ainsi que Kiewa (1999) mentionnent que l'aventure exige une totale concentration à l'égard de ses perceptions physiologiques et psychologiques. Ils expliquent que cet état de conscience sensoriel crée chez l'individu un bien-être qu'il désirera retrouver. 
l'analyse en concordance avec les principes de Paillé et Mucchielli (2008) met en évidence la pertinence de la progression de la séquence. La progression des ateliers selon la séquence d'aventure (Bilodeau, 2005a) génère ainsi des situations qui contribuent à solidifier les deux autres dimensions soit les habiletés et les connaissances. Par ailleurs, quelques commentaires des élèves mentionnent que certains comportements adoptés en ateliers sont maintenant présents dans leurs gestes quotidiens. Par exemple, prendre le temps de bien respirer pour se calmer, envisager d'aller marcher seul ou en famille dans leur temps libre, boire de l'eau plus souvent (Annexe 11).

\subsubsection{Comportements sains et sécuritaires en matière de santé dans la pratique d'activités de plein air}

L'aventure comporte des risques (Priest \& Gass, 1997), soit des dangers plus ou moins probables. Au cours de l'expérimentation, la notion de risque est présentée aux jeunes. Ils sont à différents niveaux invités à composer avec l'inconnu. Dans le nouveau paradigme de l'aventure expérientielle, le risque doit être proportionnel aux compétences des individus (Gass, 1999; Joplin, 2008). Lorsque le risque est inférieur aux compétences des participants, l'activité vécue revêt le titre d'exploration et d'expérimentation, ce qui prépare les élèves à affronter des situations plus dangereuses. La séquence d'aventure proposée aux jeunes favorise l'adoption d'un mode de vie actif et d'une conduite sécuritaire, tel que le mentionne le troisième axe du DGF Santé et bien-être du PFEQ (PFEQ, 2001), car les jeunes apprennent à 
reconnaître leurs habiletés et à tester leurs limites de façon sécuritaire. Priest (1999; voir aussi Priest \& Gass 1997) estime que la perception réelle des participants à l'égard de leur compétence ou de la tâche réduit le potentiel de risque. Cette méthode permet à l'individu de reconnaître le risque potentiel réel (Priest, 1999) et d'actualiser les compétences essentielles à la réalisation du défi à venir.

\subsection{La séquence d'aventure, l'évolution des défis éducatifs : le modèle imaginé}

L'expérimentation de la présente étude a permis de dégager certains constats qui nous ont conduits à imaginer un modèle graphique (schéma 5.6.1) pour l'implantation d'une séquence d'aventure et d'apprentissage en milieu scolaire.

L'émergence du modèle présenté au schéma 5.6.1 résulte de l'adaptation de la séquence d'aventure introduite à la figure 2.5.2. Ce modèle diffère du modèle de Bilodeau (2005a) dans le sens qu'il tient compte des principes de promotion de la santé en privilégiant une vision holistique de l'individu (Naidoo \& Wills, 1994; Tones \& Green, 2004). Le modèle graphique, présenté au schéma 5.6.1, expose l'évolution anticipée des compétences personnelles et sociales (axe de l'ordonnée) au cours de l'année scolaire (axe de l'abscisse), lors de la réalisation d'une séquence d'aventure et d'apprentissage.

Ce modèle cadre avec les besoins du milieu scolaire, car le nombre d'ateliers, ainsi que le temps accordé à leur réalisation au cours de l'année scolaire, s'ajuste à la 
séquence d'apprentissage du titulaire de classe. Le tracé continu de la courbe met en évidence l'aspect de fluidité qui réside entre la séquence d'aventure et la séquence d'apprentissage.

L'analyse des résultats à partir des axes et sous-axes du DGF Santé et bien-être nous amène à conclure en une modification d'au moins trois des facteurs clés du développement du jeune sur le plan individuel soit l'estime de soi, les compétences sociales et les habitudes de vie (annexe 1), en plus de favoriser l'acquisition de connaissances diverses.

Les propos des titulaires de classe corroborent les écrits de Lasnier (2000), à savoir que pour créer une situation authentique, l'actualisation de ce mode d'intervention doit se vivre dans une classe, où la réforme est implantée, et compter une dizaine d'ateliers.

Le modèle présenté à la figure 5.6.1, compte sept ateliers d'une demi-journée comprenant des activités de communication, de confiance, de coopération et de résolution de problèmes en suivant une progression précise (Bilodeau, 2005a; adaptation de Robb et Ewert, 1993, tiré de Bisson, 1999, p.207). L'aspect de la fluidité, représenté par la ligne continue, laisse place à une flexibilité d'intervention dans le but de coordonner la progression des apprentissages vécus en classe, et dans les ateliers d'aventure. 
Les excursions et l'expédition comportent, d'une part, des défis personnels qui se vivent en groupe (Mérini et al., 2004) et marquent, d'autre part, une évolution en intensité, c'est-à-dire des efforts physiques et psychologiques, et des risques (Priest, 1999; Gass, 1999). Dans ce contexte, à la différence de la démarche en classe, si l'un des jeunes s'arrête, c'est le groupe entier qui doit s'arrêter. Ainsi, les participants développent leurs habiletés, en ce qui a trait notamment à la gestion du stress et à l'atteinte des buts ciblés (Neill \& Richard, 1998).

La flèche transversale au cœur du graphique (schéma 5.6.1) positionne la notion d'acclimatation par l'approche sensorielle soulevée par Bilodeau (2005a). Cette notion, toujours présente, favorise :

a) La transformation de la zone de confort humain (Bilodeau, 2001; 2000; Nadler, 1993) par le développement de compétences comme structurer son identité et coopérer.

b) L'agrandissement de la zone de confort environnemental (Bilodeau, 2001; 2000; Nadler, 1993) par la compréhension de phénomènes variés et/ou par des activités qui exigent le franchissement d'obstacles environnementaux.

L'analyse des résultats montre que, tout au long de la séquence d'aventure, les jeunes s'encouragent, persévèrent, célèbrent le succès de leurs réalisations et accentuent leurs efforts. L'utilisation de leurs capacités et de leurs stratégies pour 
améliorer leur efficacité personnelle contribue, selon les écrits de Martinot (2001), à l'épanouissement de l'individu, au rehaussement de son estime et de ses compétences d'ordres personnelles et sociales.

Le schéma 5.6.1 indique donc que les derniers ateliers peuvent être propices au transfert des compétences et des apprentissages, mais ce sujet demeure à évaluer.

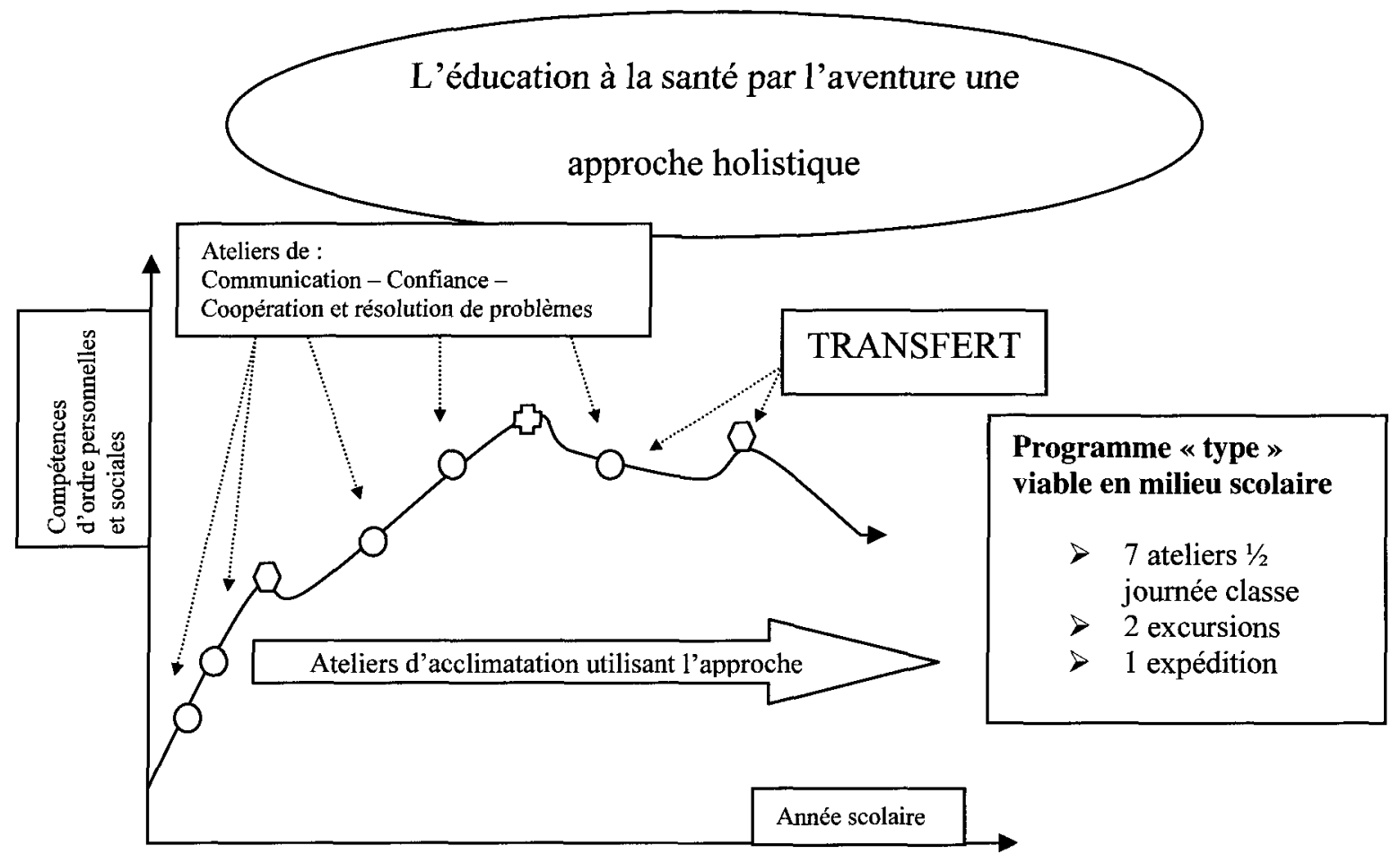

Légende

Q Ateliers $1 / 2$ journée classe

Excursion

Expédition

Schéma 5.6.1 : L'évolution anticipée des compétences personnelles et sociales au cours de l'année scolaire, lors de la mise en place d'une séquence d'aventure et d'apprentissage adoptant une approche holistique d'éducation à la santé. 


\subsection{Les pistes d'implantation}

Par manque de formation dans ce domaine, l'intégration du volet santé est généralement peu développée par les enseignants du primaire (Turcotte, 2006, Dontigny \& Roy, 2007). Aussi, l'analyse des résultats démontre que la manipulation d'instruments comme la boussole et l'approfondissement de concepts scientifiques demeurent précaires pour les titulaires de classe. Suivant ces conditions, la présence d'un coordonnateur de projet, c'est-à-dire un leader en aventure et en plein air, ou bien un spécialiste en santé (kinésithérapeute, nutritionniste ou un enseignant d'éducation physique et à la santé...) semble essentielle afin d'assurer la réussite d'un projet sur l'éducation à la santé. Il est important de souligner que l'encadrement sécuritaire des excursions et l'expédition requièrent des compétences précises de la part des intervenants scolaires. Ainsi, la mise en place de la séquence d'aventure et d'apprentissage nécessite :

- La participation des titulaires de classe pour cibler des savoirs essentiels à couvrir pour le cycle visé dans la présente étude, le troisième cycle du primaire;

- la présence d'un coordonnateur (agent de liaison) qui contribue à la planification et au suivi des ateliers d'éducation par l'aventure en plein air;

- l'engagement de l'enseignant d'éducation physique et à la santé pour parfaire le cheminement des élèves quant aux habiletés physiques, aux habitudes de vie et à l'adoption des comportements sains et sécuritaires.

- le remaniement potentiel des horaires et des tâches professorales. 
La séquence d'aventure doit servir à renforcer l'ensemble des compétences et des apprentissages ciblés par les intervenants scolaires en fonction de leur discipline respective. Par exemple, l'apprentissage de disciplines est encadré par les titulaires de classe; le développement des habiletés et des aptitudes physiques se poursuit durant les périodes réservées à l'éducation physique et à la santé; la logistique et la coordination du jumelage des deux séquences sont assurées par un coordonnateur spécialisé.

Le renouveau pédagogique et les récentes collaborations interministérielles entre le MELS et le MSSS, afin de favoriser la réussite éducative, la santé et le bienêtre des jeunes, ouvrent la porte à de nouvelles stratégies et approches pédagogiques. La séquence d'aventure et d'apprentissage expérimentée dans la présente étude réunit plusieurs conditions de réussite émises par le comité national d'orientation École en santé et ses collaborateurs (Martin \& Arcand, 2005) et peut certainement être développée dans d'autres écoles québécoises.

\subsection{Les obstacles à l'intervention}

Pour parvenir à introduire un tel mode d'intervention, il semble essentiel de présenter aux parents et acteurs du milieu scolaire les liens coexistants entre la séquence d'aventure, les apprentissages scolaires et les compétences des acteurs pédagogiques. Tous les intervenants scolaires impliqués dans le projet doivent avoir 
une vision holistique des participants et adopter une approche systémique de la situation. Cette attitude des intervenants facilite, chez les élèves, le développement de compétences et l'acquisition de connaissances.

Dans le but de faciliter la planification de la séquence d'aventure et d'apprentissage et favoriser l'acceptation auprès des parents, les cibles (compétences et apprentissages disciplinaires) du PFEQ doivent être clairement déterminées à l'avance par les titulaires de classe et connues des différents acteurs du milieu scolaire. Nous considérons que les plus importants obstacles rencontrés lors de la réalisation de la séquence d'aventure et d'apprentissage vécue au cours de la présente étude sont de type organisationnel :

- Persuader les différents acteurs du milieu décisionnel (MELS et direction d'école);

- réaménager la plage horaire;

- embaucher une personne-ressource, soit un coordonnateur de projet en éducation à la santé;

- réaliser un projet commun au sein du milieu scolaire (vision du projet éducatif, réalisation conjointe d'un même niveau de cycle, implication des intervenants à tous les niveaux). 


\subsection{Les forces et les limites de la recherche}

La force de ce mode d'intervention est de revêtir un caractère global et de proposer une éducation à la vie, tel que le recommandent Mérini et al., (2004). Les résultats obtenus démontrent que l'éducation par l'aventure marque une continuité au sein du milieu et plus précisément entre les différentes disciplines (pluridisciplinarité). Enfin, il instaure une cohérence avec les modèles d'éducation à la santé privilégiés en Amérique du Nord.

Or, l'échantillon utilisé dans la présente étude représente une limite, les résultats obtenus conviennent uniquement à la réalité des régions éloignées. Aussi, l'expérimentation effectuée se limite à une année scolaire, il est donc impossible, à ce stade, de généraliser les résultats obtenus. D’autres études devront être menées afin de valider les résultats obtenus.

Il serait intéressant d'ajouter dans la formation initiale des maîtres, plus précisément dans le cours de didactique des sciences, une formation sur l'utilisation de la boussole ou d'autres instruments scientifiques. De plus, des mises à jour concernant l'utilisation de ces instruments s'imposent, car même si cela a antérieurement été démontré dans certains cours de didactique, il est possible qu'à la suite de plusieurs années de non-utilisation, les techniques se perdent. 
L'analyse des résultats démontre que cette approche exige une planification et la collaboration de plusieurs acteurs pédagogiques. Cette situation constitue un aspect limitatif (Manidi \& Dafflon-Arvanitou, 2000) à l'implantation de la séquence d'aventure et d'apprentissage dans le milieu scolaire. Nous recommandons, pour encadrer l'évolution de la séquence, la présence d'un coordonnateur ou d'un agent de liaison afin d'orchestrer et adapter les séquences aux besoins du milieu.

\subsection{Des pistes pour de futures recherches}

Les résultats d'analyse exposent plusieurs possibilités pour induire chez les jeunes une démarche réflexive en matière de Santé et bien-être en créant des situations d'apprentissage authentiques. Dans l'optique où les enseignants éprouvent généralement de la difficulté à trouver des situations d'apprentissage qui poussent les jeunes à prendre des décisions à l'égard de leur santé et de leur bien-être (Turcotte, 2006), la séquence d'aventure et d'apprentissage est un outil d'éducation à la santé qui mérite d'être approfondi et ce, sur plusieurs facteurs de développement du jeune, tant environnementaux qu'individuels. Des études ultérieures devraient à notre avis être effectuées avec un nombre plus important de participants et étendues à plusieurs niveaux scolaires. Nous suggérons également, pour augmenter la crédibilité de l'étude, d'utiliser un groupe expérimental et un groupe témoin. Finalement, comme la séquence d'aventure et d'apprentissage semble alimenter la dynamique motivationnelle des élèves qui y participent, il serait intéressant d'évaluer son impact sur les facteurs de motivation relatifs à l'école, et à la vie en classe (Viau, 1999). 


\section{CONCLUSION}

À la lueur des résultats recueillis au cours de la présente étude, un des effets inattendus est l'apport des titulaires de classe concernant l'enrichissement du contenu, leur participation assidue aux ateliers et leur ouverture d'esprit envers la nouveauté et le partage du leardership avec la chercheure-acteure. L'acceptation tacite qu'elles ont accordée à la chercheure-acteure concernant l'animation et le déroulement de la séquence d'aventure et d'apprentissage a indéniablement eu des effets positifs sur la motivation des jeunes à l'égard du projet. Dans ces conditions, la séquence d'aventure et d'apprentissage, telle qu'elle a été exprimée, gagnerait à être considérée comme outil d'intervention en éducation à la santé en milieu scolaire, parce qu'elle :

- Développe une démarche de partenariat entre les éducateurs physiques, les titulaires de classe et les autres intervenants du milieu (Turcotte, 2006) en matière d'éducation à la santé;

- permet d'intégrer des stratégies pédagogiques recommandées dans le PFEQ: pédagogie par projet, apprentissage coopératif, tutorat par les pairs;

- favorise le développement des principaux facteurs clés du développement des jeunes soit l'estime de soi, les compétences sociales, les habitudes de vie (Martin \& Arcand, 2005); 
- utilise des outils et des méthodes qui mettent les élèves en situation d'apprentissage active (Turcotte, 2006) et authentique;

- expérimente des situations d'enseignement-apprentissage propres à l'éducation à la santé.

Également, la planification et l'application de la séquence d'aventure et d'apprentissage en milieu scolaire sont des moyens facilitant la conscientisation à l'égard d'une autonomisation de la santé et du bien-être du jeune, concept préconisé par le MEQ (2001) au primaire et au premier cycle du secondaire (MEQ, 2003). Ce mode d'intervention invite les apprenants à être actifs (Tones \& Green, 2004; Godin, 2002; Turcotte, 2006); cet élément est aussi primordial pour permettre aux élèves de développer les compétences nécessaires à l'adoption de saines pratiques en matière de santé.

D’autre part, Mérini et al. (2004) soulignent que pour y parvenir le système éducatif doit évoluer et incorporer aux stratégies pédagogiques des habiletés de vie. Les résultats obtenus par cette étude portent à croire que l'inclusion de l'activité physique au cœur des domaines d'apprentissage, à l'aide d'une séquence d'aventure et d'apprentissage, semble avoir des effets bénéfiques sur la perception des jeunes à l'égard de la pratique d'activité physique, dans leurs moments de loisir. 
La séquence d'aventure et d'apprentissage propose une approche globale qui modifie le système normatif, le système de croyance des jeunes participants (HAM, Tones \& Green, 2004) et leur donne une vision intégrale d'eux-mêmes. Les titulaires de classe qualifient cette approche expérientielle «d'éducation à la vie », ce qui assurerait aux jeunes, selon Mérini et al. (2004), une meilleure maîtrise de leur vie, de leur santé et de leur environnement et ce, tout en contribuant à soutenir la motivation des jeunes à l'égard des apprentissages et de l'école.

Finalement, nous tenons à souligner que les jeunes ayant participé à la séquence d'aventure et d'apprentissage se sont démarqués par leur participation et leur implication lors d'ateliers ultérieurs proposés par l'école. Ils ont été perçus comme des leaders positifs, démontrant de l'intérêt et de la motivation. 


\section{BIBLIOGRAPHIE}

Ann Klint, K. (1999). New direction for inquiry into self-concept and adventure experience. In Miles, J. \& Priest, S. (Ed.), Adventure programming (pp. 163-168). State College, Pennsylvanie : Venture.

Arcand, L., Daigle, J-Y., Lapointe, S., Moreau, M. \& Rodrigue, Y. (1998). Les compétences essentielles liées à la santé et au bien-être à intégrer au curriculum des enfants de l'éducation préscolaire et des jeunes du primaire et du secondaire au Québec. Groupe interministériel sur les curriculums, Volet Santé et bien-être. Québec : Gouvernement du Québec.

Ayotte, V. (1995). Évaluation d'un programme visant à développer chez les jeunes une estime positive d'eux-mêmes (Rapport de recherche). Montréal : Régie régionale de la santé et des services sociaux de Montréal-Centre.

Bardin, L. (2007). L'analyse de contenu. Paris : Presses Universitaires de France.

Barnett, T. \& Hamel, L. (2002). Activité physique. In J. Aubin, Enquête sociale et de santé auprès des enfants et des adolescents québécois 1999 (pp. 232-249). Québec : Institut de la statistique du Québec. 
Bastien, R. (2001). La mise en scène de la sortie et de la prévention dans un milieu scolaire : construction sociale d'une pratique et d'un discours. Thèse de doctorat. Montréal : Université de Montréal.

Bernier, S. \& Brochu, D. (2002). Usage de la cigarette. In J. Aubin, Enquête sociale et de santé auprès des enfants et des adolescents québécois 1999 (pp. 196-209). Québec : Institut de la statistique du Québec.

Bertrand L., Nadeau, M., Stan, S. \& Paquette, M. (2002). Comportements alimentaires. In J. Aubin, Enquête sociale et de santé auprès des enfants et des adolescents québécois 1999 (pp. 293-308). Québec : Institut de la statistique du Québec.

Beunard, B. (1999). Que pensent les lycéens de l'éducation physique? Revue Éducation Physique \& Sport, 280, 76-79.

Bilodeau, M. (1980). Le besoin du plein air dans la société et en milieu scolaire: Didactique du plein air à l'élémentaire. Manuscrit non publié. Université du Québec à Chicoutimi.

Bilodeau, M. (2000). Extrait de citations, In Souris Marie, la vie est belle. Ellesmere Island Expedition. Produit par La Fondation Sur la pointe des pieds. 52 minutes : [Film].

Bilodeau, M. (2001, automne). L'aventure thérapeutique, présentation de 1 heure. In 13th conférence annuelle. Association canadienne des infirmières en oncologie. Québec. 
Bilodeau, M. (2005a). Notes de cours, Intervention éducative et thérapeutique par la nature et l'aventure. P4PPL100. Manuscrit non publié. Université du Québec à Chicoutimi.

Bilodeau, M. (2005b). Extrait de citations, In Chute Libre. Expédition sur la Rivière Georges, Olé Gjerstad. Produit par La Fondation Sur la pointe des pieds. 53:09 minutes : [Film].

Bisson, C. (1999). Sequencing the adventure experience. In Miles, J. \& Priest, S. (Ed.), Adventure programming (pp. 205-214). State College, Pennsylvanie : Venture.

Brannon, L. \& Feist, J. (2007). Health Psychology: an Introduction to Behavior and Health. San Francisco: Wadsworth. Edition Thomson.

Brassard, J. \& Mongeau L. (2001). «Bien dans sa tête, Bien dans sa peau ». Projet de promotion d'une image corporelle saine en milieu scolaire. Rapport synthèse des années 1 et 2, 1999. Manuscrit non publié. Montréal : Collectif action alternative en obésité.

Brassard J. \& Mongeau, L. (2004). Évaluation des effets du programme «Bien dans sa tête, Bien dans sa peau " au terme de cinq années d'implantation. Manuscrit non publié. Montréal : Collectif action alternative en obésité.

Breton, J.J., Légaré, G., Laverdure, J. \& D’Amours, Y. (2002). Santé mentale. In J. Aubin, Enquête sociale et de santé auprès des enfants et des adolescents québécois 1999 (pp. 433-450). Québec : Institut de la statistique du Québec.

Boumard, P. (1996). Célestin Freinet. Paris : Presses universitaires de la France. 
Bourdieu, P. (1992). Réponses : pour une anthropologie réflexive. Paris : Éditions du Seuil.

Bruner, J.S. (1963). The process of education. Cambridge: Harvard University Press.

Byrne, B.M. \& Shavelson, R.J. (1986). On the structure of adolescent self-concept. Journal of Education Psychology, 78, 474-481.

Caflish, M. \& Paris, V. (2001), L'adolescent obèse : un casse-tête ou un défi? ProAdo. Association canadienne pour la santé des adolescents. 10 (4), 28-31.

Chabot, G. (2004). Identification des facteurs conditionnant la réussite de l'implantation de l'intervention globale et concertée en milieu scolaire primaire sur un territoire de la région 02. Mémoire de maîtrise en sciences infirmières. Université du Québec à Chicoutimi.

Chamberland, C., Dallaire, N., Fréchette, L., Lindsay, J., Hébert, J., Cameron, S. \& Beauboin, G. (1996). Promotion du bien-être et prévention des problèmes sociaux chez les jeunes et leur famille : portrait des pratiques et analyse des conditions de réussite. Division des subventions nationales au bien-être social, Ministère du Développement des ressources humaines. Canada.

Charbonneau, P. (2001). Le modèle plein air Outward Bound : Sortir des sentiers battus. L'intervenant. 17 (3), 15-17.

Cheryl, E. \& Lupton, F. (1999). The Wilderness Education Association: History and Change. In Miles, J. \& Priest, S. (Ed.), Adventure programming (pp. 77-83). State College, Pennsylvanie : Venture. 
Claes, M. (1994). L'expérience adolescente. Liège : Éditions Mardaga.

Coopersmith, S. (1967). The antecedents of self-esteem. San Francisco : Consulting Psychologists Press.

Dale, D., Corbin, C.B. \& Dale, K.S. (2000). Restricting opportunities to be active during school time: do children compensate by increasing physical activity level after school? Research Quarterly for Exercise and Sport, 71 (3), 240-248.

De Koninck, T. (2004). Philosophie de l'éducation, essai sur le devenir humain. Paris : Presses Universitaires de France.

Deschênes, C. (2001). Les habitudes de vie des élèves à risque d'échec. Mémoire de licence en sciences de l'éducation. Chicoutimi : Université du Québec à Chicoutimi.

Dontigny, D., \& Roy, J. (2007). Motivation, soutien et évaluation : les clés de la réussite des élèves. Québec : Gouvernement du Québec.

Duclos, G. (2001). Guider mon enfant dans sa vie scolaire. Montréal : Hôpital Sainte-Justine.

Duclos, G. (2000). L'estime de soi, un passeport pour la vie. Montréal : Hôpital Sainte-Justine.

Dugual, S. (1999). Rapport sur la problématique des jeunes filles et la pratique de l'activité physique (Rapport de recherche). Kino-Québec.

Écuyer, L', R. (1994). Le développement du concept de soi de l'enfance à la vieillesse. Montréal : Presse de 1'Université de Montréal. 
Famose, J.P. (2001). La motivation en éducation physique et en sport. Paris : Édition Armand Colin.

Ford, P. (1986). Outdoor Education: Definition and philosophy, ERIC Clearinghousse on Rural Education and Small Schools Las Cruces NM. (pp. 1-4).

Gagné, C., Belley, G., Legault, C. \& Samson, G. (2003). Aventure, expéditions au cour de la science et de la technologie. Primaire, troisième cycle. Guide d'enseignement. Fascicule 1. Montréal : Édition Lidec.

Garnier, C., Bednarz, N. \& Ulanovskaya, I. (1991). Après Vygotski et Piaget: perspectives sociales et constructivistes : l'école russe et occidentale. Bruxelles : De Boeck-Wesmael.

Gass, M.A. (1993). Foundations of adventure therapy. In M.A. Gass, Adventure therapy. États-Unis: Kendall/Hunt.

Gass, M.A. (1999). Tranfer of learning. In Miles, J. \& Priest, S. (Ed.), Adventure programming (pp. 227-234). State College, Pennsylvanie : Venture.

Godin, G. (2002). Le changement des comportements de santé. In Fischer G-N, dir. Traité de psychologie de la santé (pp. 375-388). Paris: Dunod.

Godin, G. (2006). Enquête sur la pratique de l'activité physique chez les jeunes en milieu scolaire (Rapport de recherche, présenté à la commission scolaire des Découvreurs). Québec: Université de Laval.

Grenier, J. (1977, janvier). Une définition: L'éducation en plein air, Québec nature. 
Gueyaud, J.-A. \& Dassa, C. (1998). La configuration des corrélations entre le concept de soi et le rendement scolaire: une méta-analyse. Revue des sciences de l'éducation, 24 (2), 299-322.

Hamel, M. (2001). Le concept de soi ou 1'estime de soi. In Hamel, M.L., Blanchet, L. \& Martin, C. 6-12-17 nous serons bien mieux! Les déterminants de la santé et du bien-être des enfants d'âge scolaire (pp. 133-168). Sainte-Foy: Publications du Québec.

Hattie, J., Marsh, H.W., Neill, J. \& Richards, G.E. (1997). Adventure Education and Outward Bound : Out-of-Class Experiences That Make a Lasting Difference. Review of Educational Research, 67 (1), 43-87.

Henton, M. (1996). Adventure in the classroom: using adventure to strengthen learning and build a community of life-long learners. États-Unis : Kendall/Hunt.

Herry, Y. \& Worth Gavin, D.A. (1996). Polarité et congruence du concept de soi chez les élèves de 9 à 12 ans. Science et comportement, 25 (2), 223-238.

Hirsch, J. (1999). Developmental Adventure Programs. In Miles, J. \& Priest, S. (Ed.), Adventure programming (pp. 13-27). State College, Pennsylvanie : Venture.

Hopkins, D. \& Putnam, R. (1997). Personal growth though adventure. Londres : David Fulton publishers.

Horwood, B. (1999). Educational Adventure and Schooling. In Miles, J. \& Priest, S. (Ed.), Adventure programming (pp. 9-12). State College, Pennsylvanie : Venture. 
Houssaye, J. (1994). Quinze pédagogues, leur influence aujourd'hui : Rousseau, Pestalozzi, Fröbel, Robin, Ferrer, Steiner, Dewey, Decroly, Montessori, Makarenko, Ferrière, Cousinet, Freinet, Neill, Roger. Paris : Éditions Armand Colin.

Institut national de la santé et de la recherche médicale (Inserm). (2001). Éducation pour la santé des jeunes : démarches et méthodes. Paris : Les éditions Inserm.

Insel, P., Roth, W., Petersen, R., Rollins, M. \& Petersen, R. (1997). Core Concepts in Health: Brief. Mayfield : Pub Co. Date Published.

Institut national de santé publique du Québec. (2004). Huitième Journées annuelles de santé publique. Sur tous les fronts bâtir la santé. [www.inspq.qc.ca/jasp].

Institut national de santé publique du Québec. (2009). Analyse des interventions de promotion de la santé et de prévention en contexte scolaire québécois : cohérence avec les meilleures pratiques selon 1'approche. [www.inspq.qc.ca].

Jasper, S.H.Jr. \& Wurdinger, S.D. (1999). Philosophy of Adventure Education. In Miles, J. \& Priest, S. (Ed.), Adventure programming (pp. 155-122). State College, Pennsylvanie : Venture.

Joplin, L. (2008). On Defining Experiential Education. In Warren, K., Mitten, D., \& Loeffler, TA. (Ed.), Theory \& Practice of Experiential Education (pp. 16-23). United States : Association for experiential education.

Jutras, S. \& Bisson, J. (1994). La conception de la santé chez des enfants de 5 à 12 ans. Quelques clés pour la promotion de la santé. Sciences Sociales et Santé, 7 (2), 537. 
Karsenti, T. \& Savoie-Zajc, L. (2004). La recherche en éducation: étapes et approche. Sherbrooke : Édition du CRP, Université de Sherbrooke.

Kiewa, J. (1999). Kinesthetic Awareness : At Home in Our Bodies, In Miles, J. \& Priest, S. (Ed.), Adventure programming (pp. 353-356). State College, Pennsylvanie : Venture.

Kraft, R.J. (1999). Experiential Learning, In Miles, J. \& Priest, S. (Ed.), Adventure programming (pp. 181-186). State College, Pennsylvanie : Venture.

Lasnier, F. (2000). Réussir la formation par compétences. Montréal : Guérin éditeur.

Lapointe, N., Dubois, N. \& Lapointe, N. (2000). Enquête concernant la pratique d'activités physiques chez les jeunes de niveau scolaire de $\sigma^{e}$ année du primaire et $1^{r e}$ année du secondaire (Rapport). Régie régionale de la santé et des services sociaux des Laurentides.

Ledoux, J. (2002). Le programme "Bien dans sa tête, Bien dans sa peau»: Guide d'intervention sur le poids et l'image corporelle auprès des jeunes en milieu scolaire. Montréal : Collectif action alternative en obésité.

Ledoux, M., Mongeau, L. \& Rivard, M. (2002). Poids et image corporelle. In J. Aubin, Enquête sociale et de santé auprès des enfants et des adolescents québécois 1999 (pp. 311-339). Québec : Institut de la statistique du Québec.

Legendre, R. (2005). Dictionnaire actuel de l'éducation. Montréal : Guérin éditeur.

Manidi, M.-J. \& Dafflon-Arvanitou, I. (2000). Activité physique et santé : apport des sciences humaines et sociales, éducation à la santé par l'activité physique. Paris : Masson Paris. 
Martin, C. \& Arcand L. (2005). Guide à l'intention du milieu scolaire et de ses partenaires. Pour la réussite éducative, la santé et le bien-être des jeunes. École en santé. Québec : MELS.

Martinot, D. (2001). Connaissance de soi et estime de soi : ingrédients pour la réussite scolaire. Revue des Sciences de l'éducation, 27(3), 483-502.

Maslow, A. (1972). Vers la psychologie de l'être. Paris : Éditions Fayard.

Maslow, A. (2004). Accomplissement de soi - De la motivation à la plénitude. Paris : Éditions d'Organisation.

Mérini, C., Jourdan, D., Victor, P., Berger, D. \& De Peretti, C. (2004). Guide ressource pour une éducation à la santé à l'école élémentaire. Rennes : Éditions ENSP.

Miles, J. (1999). Adventure programming. State College, Pennsylvanie : Edited by John Miles and Simon Priest.

Ministère de l'Éducation du Québec (MEQ). (1981). Programme d'études. Secondaire. Éducation physique. Québec : Gouvernement du Québec.

Ministère de l'Éducation du Québec (MEQ). (1996). Les États généraux sur l'éducation, 1995-1996 : Exposé de la situation. Québec : Gouvernement du Québec.

Ministère de l'Éducation du Québec (MEQ). (2001). Programme de formation de l'école québécoise. Éducation préscolaire, enseignement primaire (PFEQ). Québec : Gouvernement du Québec. 
Ministère de l'Éducation du Québec (MEQ). (2003). Programme de formation de l'école québécoise. Enseignement secondaire, premier cycle. Québec: Gouvernement du Québec.

Ministère de la Santé et des Services sociaux (MSSS). (1997). Priorités nationales de santé publique, 1997-2002. Québec : Bibliothèque nationale du Québec.

Morissette, P., Dedobbeleer, N. \& Rojas Viger, M. (2002). Consommation de substances psychoactives. In J. Aubin, Enquête sociale et de santé auprès des enfants et des adolescents québécois 1999 (pp. 211-229). Québec : Institut de la statistique du Québec.

Motta, D. (1998). Éducation pour la santé et disciplines scolaires : Réflexions sur les expériences américaines et britanniques. Recherche et Formation, 28, 79-99.

Nadler, R.S. (1993). Therapeutic process of change. In Gass. M. A. (Eds.), Adventure therapy (pp. 57-69). États-Unis : Kendall/Hunt Publishing company.

Naidoo, J. \& Wills, J. (1994). Health Promotion, foundations for practice. Londres $(\mathrm{RCN})$ : Baillière Tindall.

Neill, J. (2000). Building healthy challenges for adolescent development. Presentation to Applied Psychology Symposium «Partnership in Promoting Young People's Health in the ACT », University of Canberra, Australia. $13^{\text {th }}$ june.

Neill, J. \& Richard, G.E. (1998). Does Outdoor Education Really Work? A Summary Of Recent Meta-Analyses. Australian Journal of Outdoor Education. 3 (1), 1-9. 
Ouellet, M. \& Stan, S. (2002). Milieu_scolaire et caractéristiques des jeunes à l'école. In J. Aubin, Enquête sociale et de santé auprès des enfants et des adolescents québécois 1999 (pp. 109-131). Québec : Institut de la statistique du Québec.

Parcel, G. S., Kelder, S. H. \& Basen-Engquist, K. (2000). The School as a Setting for Health Promotion. In Poland, B. D., Green, L. W. \& Rootman, I. (2000). Settings for Health Promotion. Sage : Londres.

Parent, A-M., Munroe, D.C., Filion, G., Lapointe, J., LaRoque, P., Mcllhone, J., Rocher, G., De Rome, M-L. \& Tremblay, A. (1964). Rapport de la Commission royale d'enquête sur l'enseignement dans la province de Québec. Deuxième partie ou tome II (suite): Les structures pédagogiques du système scolaire. Québec: Gouvernement du Québec.

Paillé, P. \& Mucchielli, A. (2008). L'analyse quanlitative en science humaines et sociales. Paris : Armand Colin.

Priest, S. \& Gass, M.A. (1997). Effective leadership in adventure programming. Champaign : Human Kinetics.

Priest, S. (1999). The semantics of adventure programming. In Miles, J. \& Priest, S. (Ed.), Adventure programming (pp. 109-114). State College, Pennsylvanie : Venture.

(Promotion de la santé). (1986). Charte d'Ottawa, Promotion de la santé. Première conférence internationale pour la promotion de la santé, 1(4), iii-v.

Pronovost, G. (2002). Emploi du temps et pratiques culturelles. In J. Aubin, Enquête sociale et de santé auprès des enfants et des adolescents québécois 1999 (pp. 255268). Québec : Institut de la statistique du Québec 
Rabiola, E. \& O'Keefe, M. (1999). Philosophy in Practice: A History of Adventure Programming. In Miles, J. \& Priest S. (Ed.), Adventure programming (pp. 45-52). State College, Pennsylvanie : Venture.

Reasoner, R.W. (1982), Building Self-Esteem, Elementary Edition. Palo Alto, California: Consulting Psychologists Press inc.

Rey, A., Rey-Debove, J. \& Cottez, H. (1990). Petit Robert 1. Canada: Éditions Dictionnaire Robert - Canada.

Rosenberg, M. (1979). Conceiving the self. New York : Basic Books.

Rosenstock, I.M. (1974). Historical Origins of the Health Belief Model, Healh Education Monographs, 2, 328-335.

Sallis, J.F. \& Owen, N. (1999). Physical Activity and Behavioral Medicine, Thousand Oaks. États-Unis : Sage.

Sandrin-Berthon, B. (1997). Apprendre la santé à l'école. Collection pratiques \& enjeux pédagogiques. France : CFES (Comité français d'éducation pour la santé).

Savoie-Zajc, L. (2001). La recherche-action en éducation: ses cadres épistémologiques, sa pertinence, ses limites. In Anadon, M. Nouvelles dynamiques de recherche en éducation (pp. 15-49). Québec : Presses de l'Université Laval.

Schaefer C. \& Mongeau, L. (2000). L'obésité et la préoccupation excessive à l'égard du poids. Montréal : CAAO.

Shavelson, R. J., Hubner, J.J., \& Stanton, G.C. (1976). Validation of constructs interpretations. Review of Educational Research, 46, 407-441. 
Suzuki, D. (2001). Équilibre sacré. Montréal : Éditions Fides.

Thibault, G. (2000). L'activité physique, déterminant de la santé des jeunes. Avis du comité scientifique de Kino-Québec.

Thibault, G. (2001). L'activité physique. In Hamel, M.L., Blanchet, L. \& C. Martin, 6-12-17 nous serons bien mieux! Les déterminants de la santé et du bien-être des enfants d'âge scolaire (pp. 211-250). Sainte-Foy : Publications du Québec.

Tones, K. \& Green, J. (2004). Health Promotion, Planning and Strategies. Londres: SAGE publications Inc.

Toussaint, R. \& Lavigne, A. (2001). Apprentissage et enseignement des sciences et de la technologie au primaire. Boucheville, Québec : Gaëtan Morin éditeur.

Turcotte, S. (2006). L'inclusion de l'éducation à la santé en éducation physique : analyse des pratiques pédagogiques d'éducateurs physiques du primaire. Thèse de doctorat en sciences de l'éducation. Université du Québec à Montréal.

Viau, R. (1999). Pour une catégorisation des facteurs environnementaux qui influencent la motivation des élèves en contexte scolaire. Apprentissage et socialisation, 19 (2), 65-79.

Vygotski., L.S. (1985). Pensée et langage. Paris : Éditions sociales.

Ziller, R.C. (1973). The social self. N.Y : Pergamon Elmsford. 


\section{ANNEXES}

Annexe 1 Schémas des facteurs clés du développement, AES (Martin \& Arcand, 2005)

Annexe 2 Modèle multidimensionnel et hiérarchique du concept de soi

Annexe 3 Tree theories of transfert in Adventure Education (Gass, 1999)

Annexe 4 Lettre de consentement aux parents

Annexe 5 Exemple de fiche d'ateliers : Ateliers 2

Annexe 6 Fiches de retour des ateliers 1 à 10 et de la réalisation du projet

Annexe 7 Canevas d'entrevue pour les titulaires de classe

Annexe 8 Canevas d'entrevue pour l'enseignante d'éducation physique et à la santé

Annexe 9 Questionnaire pour la direction de l'école Fréchette

Annexe 10 Questionnaire destiné aux parents

Annexe 11 Tableaux synthèse des instruments de mesures

Annexe 12 Demande d'intervenants stagiaires en plein air et touriste d'aventure de I'UQAC. 


\section{Annexe 1}

Schémas des facteurs clés du développement, AES (Martin \& Arcand, 2005) 
Les facteurs clés du développement des jeunes

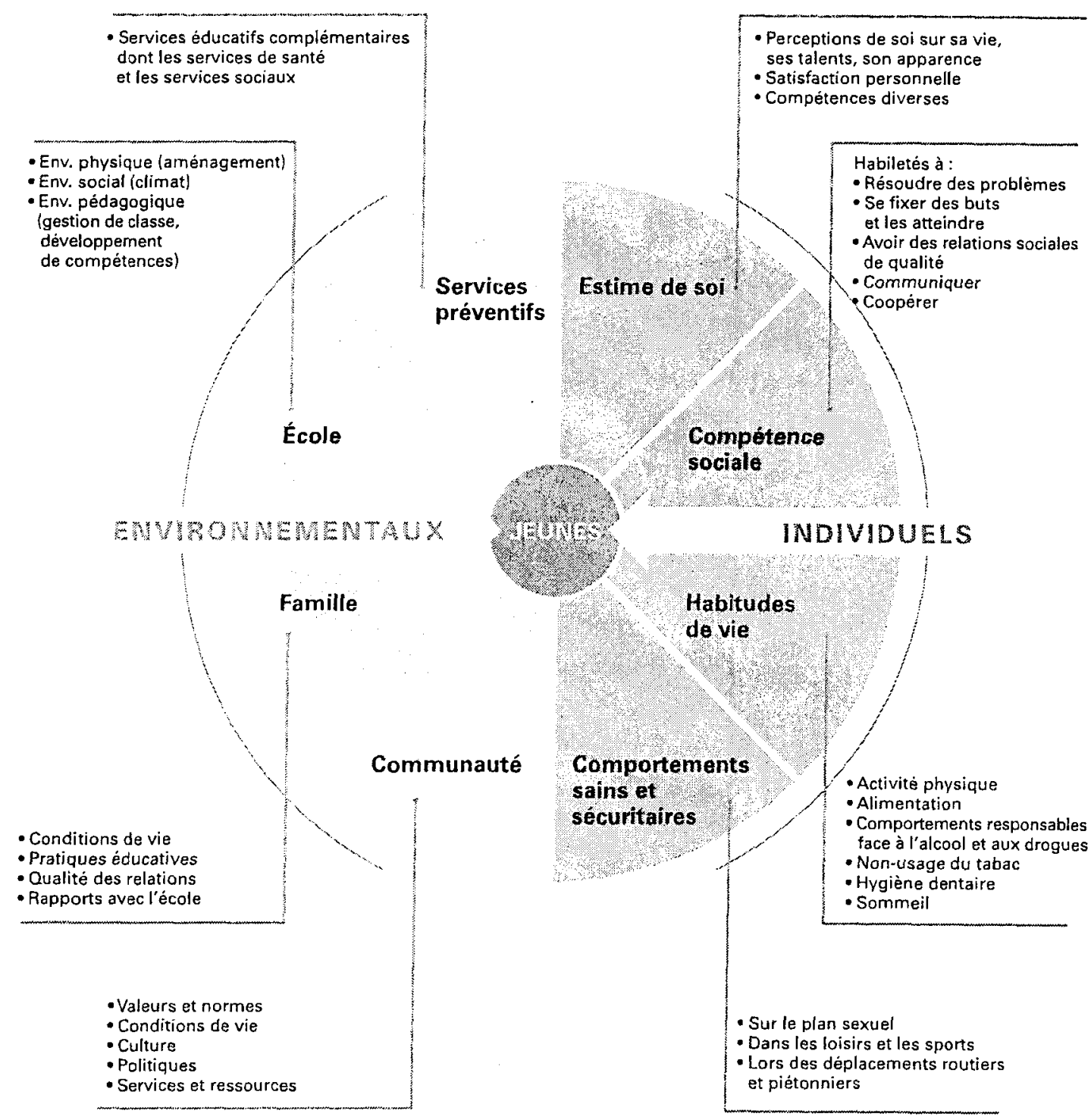




\section{Annexe 2}

\section{Modèle multidimensionnel et hiérarchique du concept de soi}

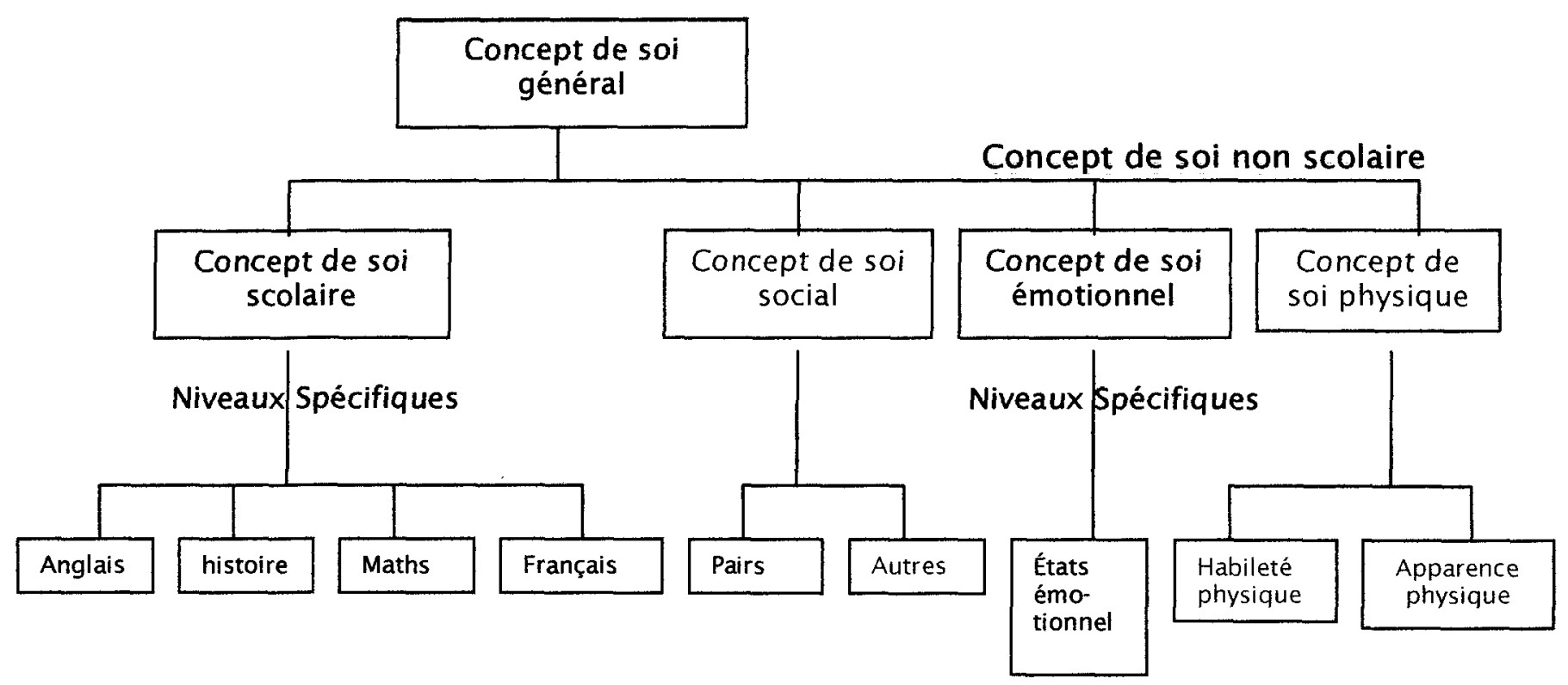

Adapté de Shavelson, Hubner et Stanton (1976) 


\section{Annexe 3}

Tree theories of transfert in Adventure Education (Gass, 1999) 
Figure 31.1 Three Theories of Transfer in Adventure Education

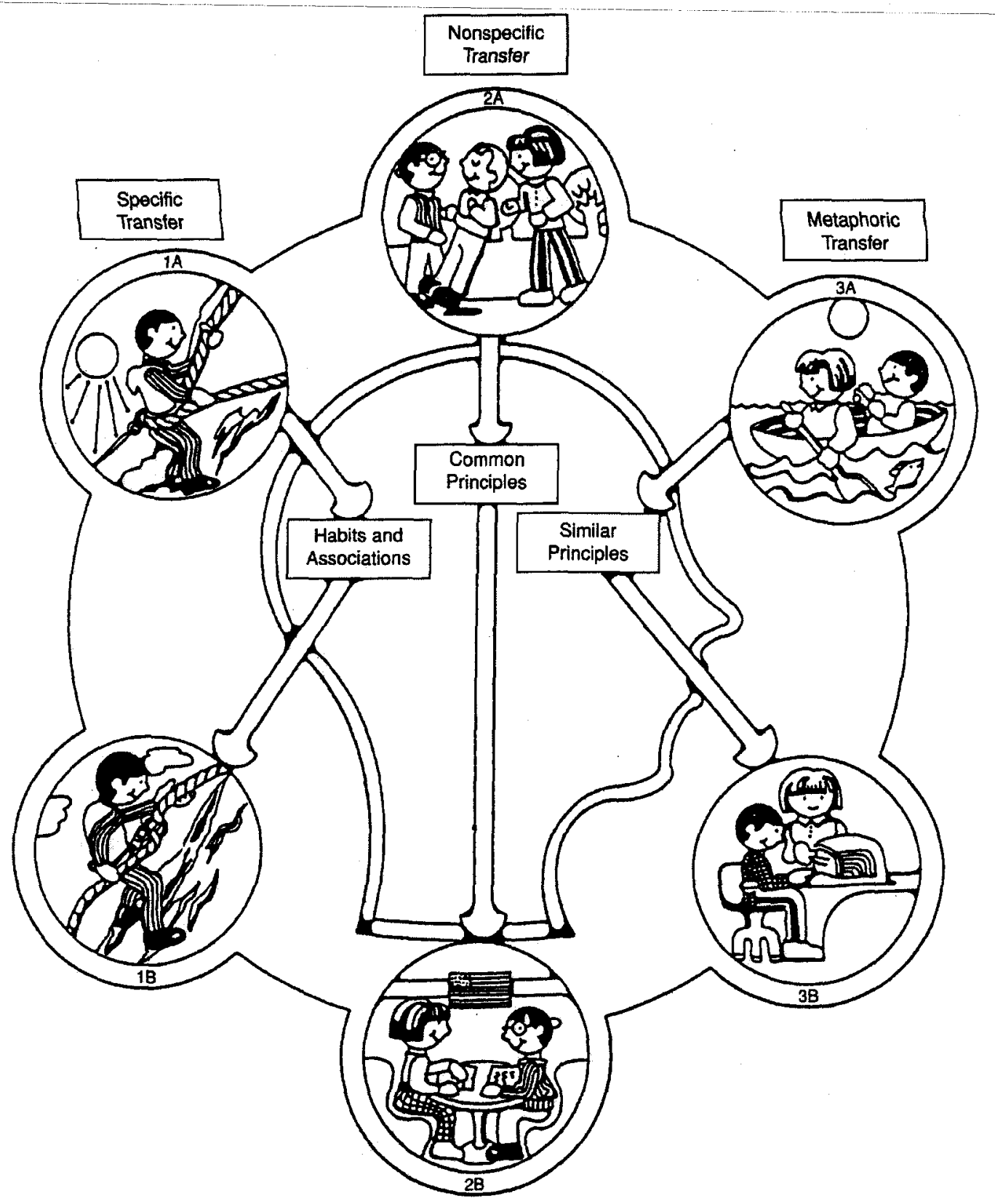

The above diagram illustrates how learning in adventure education is linked to future learning experiences. In the first theory, specific transfer, the learner takes the habits and associations acquired during a previous experience (Diagram $1 A$-the hand skills of belaying) and applies them to a new experience to assist him in developing a new skill Clagram 1B-the hand skills of rappelling.) In the second theory, nonspecific transfer, the learner generalizes the

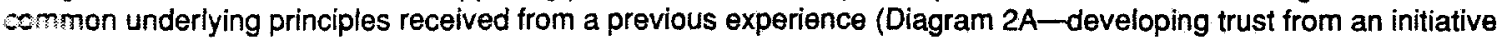
pame) and employs them in a new learning situation (Diagram $2 \mathrm{~B}$-developing trust with peers at school). The third teory, metaphoric transter, shows the learner transferring the similar underlying principles from canoeing (Diagram 34 to working with other individuals in a business corporation (Diagram 3B).

Saros: Gass. 1902. 2. 202 Fepinted with permission of Venture Pliblishing. Inc. 
Annexe 4

Lettre de consentement aux parents 
9 octobre, 2003

\section{Objet : $\quad$ Consentement des parents concernant la participation des élèves du $3^{\mathrm{e}}$ cycle du primaire à participer au projet Nature et santé}

Chers parents,

La présente est pour vous informer que votre enfant aura l'opportunité de participer à une étude concernant l'estime de soi chez les jeunes. Dans un premier temps, nous lui ferons passer un questionnaire de façon confidentielle et il sera uniquement utilisé par un chercheur de l'Université du Québec à Chicoutimi.

Aussi, dans le but d'améliorer les activités d'apprentissage et de stimuler la participation des élèves, chaque atelier sera suivi d'une fiche de retour présentée comme des travaux scolaires orientés qu'il devra compléter individuellement au meilleur de ses connaissances.

Afin de permettre à votre enfant de participer à cette étude, nous vous demandons par la présente votre consentement écrit.

Merci de votre collaboration.

Josiane Gagnon, Dt. P.

Diane Gauthier, Ph. D.

Étudiante à la maîtrise à l'UQAC

Professeure à l'UQAC

J'accepte que mon enfant participe à l'étude : Oui ___ Non

Nom de l'enfant :

Signature :

S.V.P. veuillez retourner cette partie à l'école 


\section{Annexe 5}

\section{Exemple de fiche d'ateliers}




\section{$\underline{\text { A2 - Randonnée et jeux coopératifs }}$}

\section{Les thèmes}

1. Entraide et coopération

2. Exploitation des habiletés corporelles.

3. Survol du lien entre l'écosystème terrestre et le corps humain.

\section{L'atelier}

Faire participer les jeunes à des activités de coopération et d'entraide. Les sensibiliser à l'importance de la collaboration qui existe sur la terre, entre eux, mais aussi avec les éléments (eau, terre, air).

\section{Les objectifs à générer}

- Favoriser la coopération avec les pairs;

- Concevoir la place de l'humain dans l'écosystème terrestre.

\section{Domaines de formation}

Santé et bien-être : conscience de soi et de ses besoins fondamentaux.

Environnement et consommation : construction d'un environnement viable dans une perceptive de développement durable.

\section{Compétences transversales}

D'ordre intellectuel :

Résoudre des problèmes:

- L'élève sera capable de mettre en valeur ses forces.

Mettre en ceuvre sa pensée créatrice:

- L'élève sera en mesure d'organiser un plan d'action. 
D'ordre de la communication :

- L'élève devra s'approprier un vocabulaire précis et l'utiliser de façon appropriée.

D'ordre personnel et social :

Structure son identité :

- L'élève sera en mesure de percevoir sa place dans l'équilibre de l'écosystème et parmi les autres.

Coopérer :

- L'élève devra contribuer au travail collectif de façon active et accomplir sa tâche selon les règles établies par le groupe.

\section{Domaines d'apprentissage}

\section{Mathématique, science et technologie :}

- Interaction entre l'humain et son milieu (piste cyclable, forêt boréale...)

- L'Univers vivant

- pyramides alimentaires

- compréhension de l'écosystème

- La terre et l'espace :

- phénomène naturel; rivière.

\section{Langue :}

- Communiquer oralement : exprimer sa pensée de façon cohérente et structurée en utilisant un vocabulaire adapté à la situation d'apprentissage, soit l'écosystème.

Développement personnel/éducation physique et à la santé :

- Adopter un mode de vie actif : respecter les règles de sécurité et adapter son comportement aux situations dangereuses. 


\section{Matériel nécessaire}

- Balle de laine

- Images d'éléments de l'écosystème (eau, terre, animaux, champignons, insectes, plantes, etc.)

- Corde de 15 mètres

\section{Préparation en classe}

Cette section présente aux titulaires de classe un angle de traitement didactique qui permet d'ouvrir la porte à une activité d'apprentissage qui sera effectuée (ou a été effectuée) lors de l'atelier d'aventure en plein air. Cette étape est primordiale afin de tisser les liens entre la séquence d'aventure et la séquence d'apprentissage. La préparation en classe est généralement effectuée par le titulaire de classe.

1. Voir en classe les quelques éléments théoriques de l'écosystème (Toussaint \& Lavigne, 2001, p.57, activité 4.3). L'écosystème et l'interdépendance des êtres vivants.

2. Demander aux élèves de trouver une image de leur choix représentant un élément de l'écosystème. L'image doit être cartonnée (ou plastifiée) et couvrir une surface d'au moins $60 \mathrm{~cm}^{2}$.

\section{Déroulement de l'atelier}

Il est à noter que les activités présentées ci-dessous contribuent à créer un climat propice aux apprentissages. Pour l'atelier 2, les activités présentées aux jeunes devraient favoriser le développement des habiletés de l'ordre de la communication et de la résolution de problèmes. 
Jeux de réchauffement

Ensemble on se lève : En équipe de 2, les élèves doivent se placer dos à dos et se tenir par les coudes, puis tenter de se lever. Lorsqu'ils ont réussi, le défi est lancé de l'essayer à 4,8 et 16 ... selon la grosseur du groupe

Passe la passe : Sur un grand terrain, diviser la classe en deux groupes, puis identifier les équipes à l'aide de foulard. Chacun des membres de l'équipe doit trouver une place de son choix sur le terrain. Lorsque les joueurs sont éparpillés, donner le ballon à un des joueurs. Au signal, l'équipe possédant le ballon doit se le passer afin que chaque membre de l'équipe y touche avant de se le faire intercepter par l'autre équipe. Tous les membres ont le droit de se déplacer.

\section{Activité d'apprentissage réalisée en plein air portant sur l'écosystème}

Existe-t-il une coopération ou une collaboration entre les éléments qui le composent?

Définition : l'écosystème est une unité écologique de base formée par le milieu vivant et les organismes animaux et végétaux qui y vivent.

Exercice : Distribuer un carton représentant un élément de l'écosystème (eau, plante, champignon, animal, insecte) à chaque élève. Donner une balle de laine à l'un d'entre eux, puis demander à cet élève de lancer la balle à un ami qui possède un carton représentant un élément en lien avec le sien (ex : poissons et algues). Poursuivre le déroulement jusqu'à ce que tous les regroupements imaginés par les jeunes soient effectués.

À la suite de l'activité d'apprentissage vécue en classe, l'intervenant questionne les jeunes sur leurs connaissances à l'égard de l'écosystème. Voici des exemples de questions :

- Quelle est l'importance des éléments (l'air, l'eau et la terre) dans la survie de la plupart des organismes vivants?

- D'où provient l'énergie nécessaire à la croissance des organismes vivants? 


\section{Aide mémoire pour l'intervenant et lien avec le fonctionnement physiologique}

La fonction primaire de l'écosystème est la circulation de l'énergie et la circulation des substances nutritives. Dans la chaîne alimentaire, on retrouve généralement trois sortes d'organismes : producteurs, consommateurs et décomposeurs.

Par exemple: Une plante qui consomme l'énergie du soleil pour la convertir en nourriture est producteur. Un animal qui se nourrit de la plante est consommateur tandis qu'une bactérie (ou autre organisme) aidera à la décomposition d'un animal (ou d'une plante morte) afin de redonner des éléments nutritifs à la terre.

Établir un lien avec 1'humain : est-il producteur ou consommateur? Peut-il modifier l'écosystème par ses gestes quotidiens? D'où provient l'énergie nécessaire à sa survie (sous quelle forme retrouve-t-on cette énergie (fruits et légumes, céréales, fromage)? Comment le corps humain utilise-t-il cette énergie?

Variante : Demander aux élèves concernés par la mise en situation de lâcher le fil.

Discuter ensuite avec les élèves de leur apprentissage.

\section{Utiliser les forces de chacun pour un travail d'équipe :}

Installer entre deux arbres une corde à environ 1,2 mètre du sol (pour l'installation respecter les principes de leave no trace).

Tous du même côté de la corde, les jeunes se placent en équipe de deux, puis effectuent un jeu de pouce (deux par deux). Les perdants du jeu doivent passer de l'autre côté de la

corde. À la fin, le vainqueur se retrouvera seul de son côté alors que l'ensemble du groupe se retrouvera du côté opposé de la corde. 
Le but devient alors de trouver une astuce pour que tous les jeunes traversent de l'autre côté de la corde et rejoignent le vainqueur en passant par-dessus celle-ci sans la toucher. Ils auront droit d'utiliser trois objets de leur choix; souche, roche... La collaboration et utilisation du jugement critique est de mise afin d'arriver rapidement au but.

Après l'atelier, discuter de leur expérience.

\section{Bibliographie :}

Daniel, K.S. (2000) Notes de cours protection de l'environnement AEC en tourisme d'aventure. Cégep de Saint-Laurent.

Toussaint, R. et Lavigne, A. (2001) Apprentissage et enseignement des sciences et de la technologie au primaire. Gaëtan Morin éditeur, 278 pages.

Questions d'objectivation : document ci-joint - Fiche de retour : Atelier 2. (Annexe 6). 


\section{Annexe 6}

Fiches de retour des ateliers 1 à 10 et de la réalisation du projet 
Fiche de retour

\section{Activité 1 - Nature et santé}

Nom :

Date:

Objet choisi :

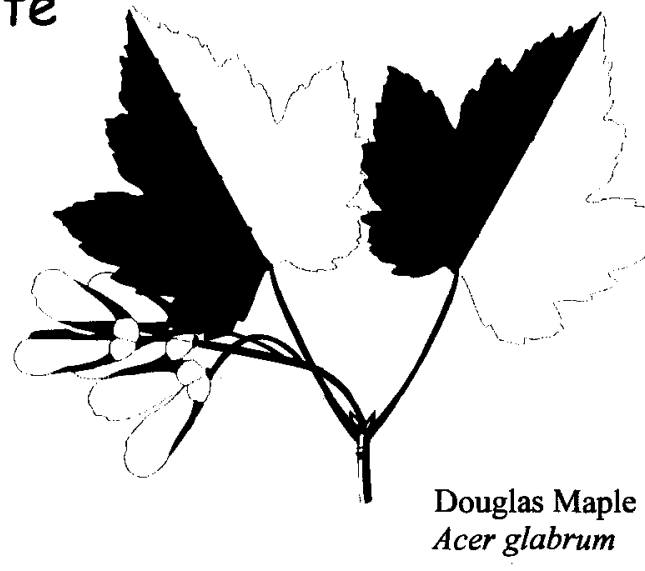

1. Connaissais-tu cet objet avant de le choisir?

2. Raconte-moi comment tes sens (l'ouie, la vue, etc.) t'ont attiré vers l'objet que tu as choisi?

3. Pourquoi as-tu préféré cet objet à un autre?

4. Explique-moi comment cet objet peut te représenter ou comment tu te retrouves dans cet objet?

\footnotetext{
${ }^{1}$ Josiane Gagnon, projet Nature et santé.
} 
Fiche de retour

\section{Activité 2 - Nature et santé ${ }^{2}$}

Nom :

Date :

1. Selon toi, quel(s) lien(s) as-tu avec la nature?

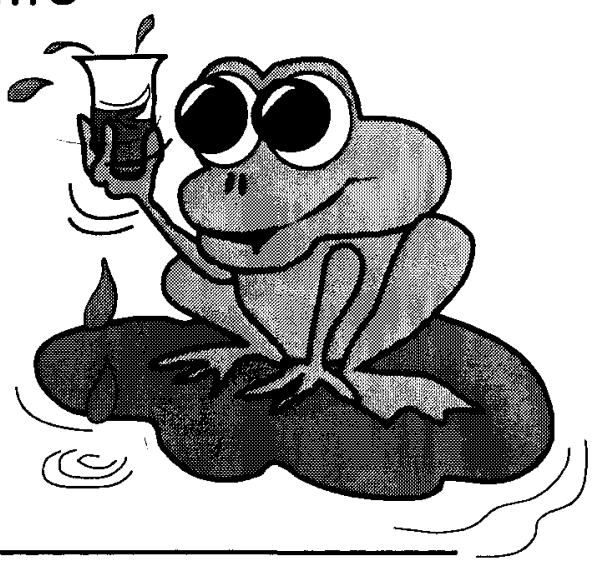

2. Nomme-moi trois partenaires dans la nature:

a. Un producteur :

b. Un consommateur :

c. Un décomposeur :

3. Crois-tu important de collaborer avec les autres?

Oui : Non :

Pourquoi?

\footnotetext{
${ }^{2}$ Josiane Gagnon, projet Nature et santé.
} 
Fiche de retour

\section{Activité 3 - Nature et santé 3}

Nom :

Date:

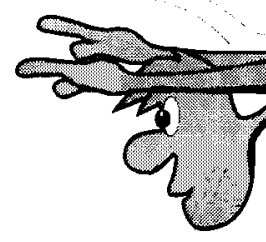

1. Explique-moi à la lueur des exercices que nous avons faits ensemble, comment perçois-tu ton corps? 


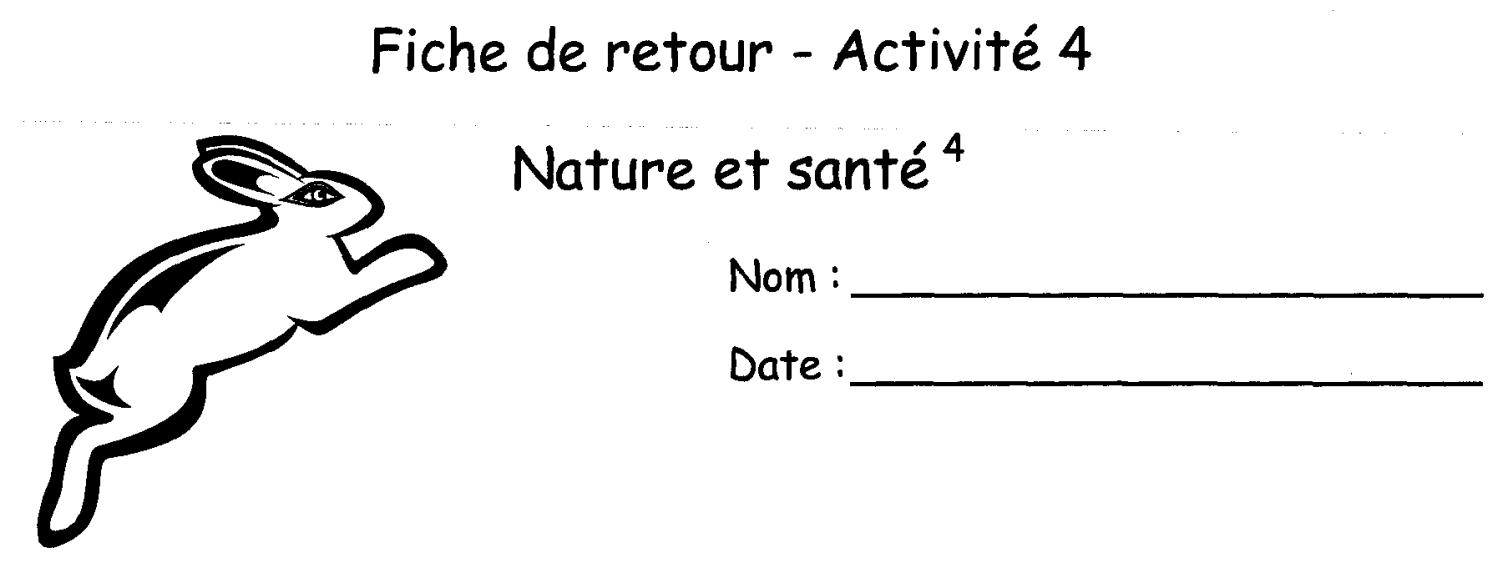

1. Au cours de la randonnée à la chute, nous avons vu de l'eau sous différentes formes. Nomme-moi trois (3) ÉTATS différents où tu as pu observer l'eau lors de la randonnée :

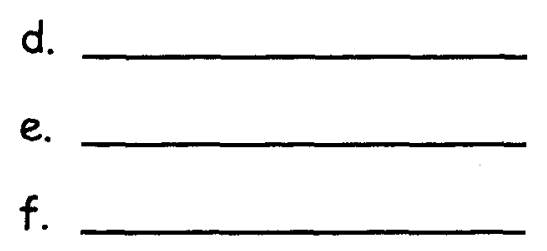

2. Selon toi, l'eau est-elle essentielle pour ton organisme? Oui_ Non

Explique-moi pourquoi?

3. Outre l'humain, nomme-moi deux organismes vivants qui ont besoin d'eau pour croître (grandir) ou tout simplement pour vivre :

g.

h.

${ }^{4}$ Josiane Gagnon, projet Nature et santé. 
Fiche de retour

\section{Activité 5 - Nature et santé 5}

Nom:

Date :

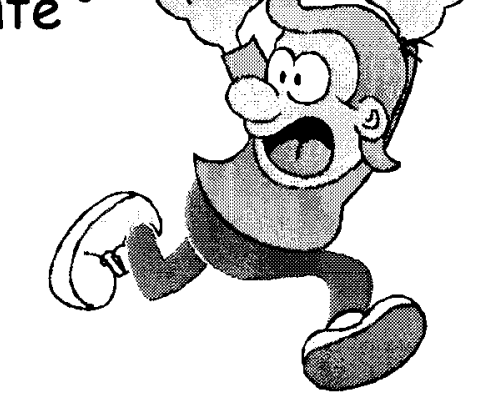

Décris-moi quatre bruits (ou sons) que tu as entendus au cours de l'activité et à quelle(s) occasion(s):

1.

2.

3.

4.

Explique-moi les difficultés que tu as ressenties lors de l'activité du cerceau. (Problème d'équilibre, difficulté à trouver ta place au centre du groupe, etc.)

${ }^{5}$ Josiane Gagnon, projet Nature et santé. 
Fiche de retour

\section{Activité 6 - Nature et santé 6}

Nom :

Date :

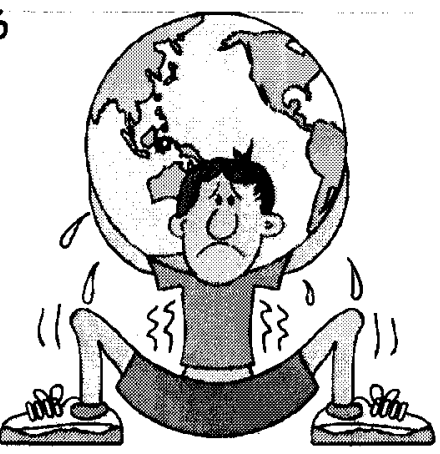

Ton corps possède plusieurs habiletés physiques (force, souplesse, agilité, endurance, dextérité, adresse, précision, équilibre, etc.). Nomme-moi trois habiletés qui t'ont été utiles pour la réalisation des défis proposés lors de l'activité 6, puis explique-moi pourquoi.

1.

2.

3.

Lors de la chorégraphie (le minisketch), comment as-tu trouvé l'échange avec tes partenaires? As-tu rencontré des difficultés à communiquer?

Préfères-tu le chocolat contenant $70 \%$ de cacao ou le chocolat au lait?

Pourquoi?

\footnotetext{
${ }^{6}$ Josiane Gagnon, projet Nature et santé.
} 


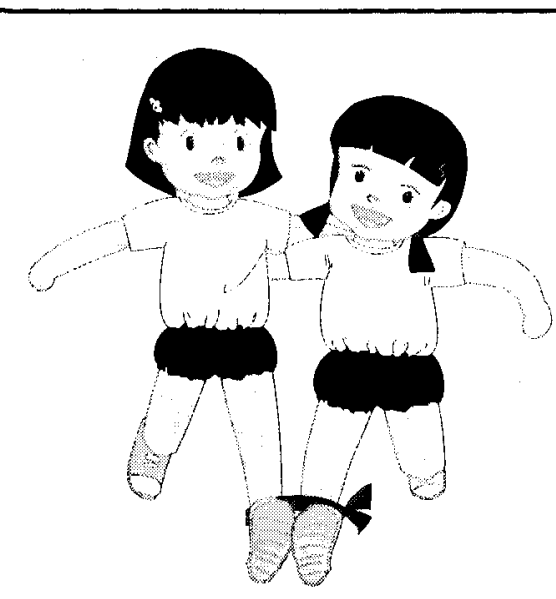

Fiche de retour - Activité 7

Nature et santé 7

Nom :

Date :

1. Au centre du cercle, lorsque tu avais les yeux bandés, comment t'es-tu senti(e)?

2. As-tu trouvé facile ou difficile de faire confiance aux autres? Facile Difficile

Explique-moi pourquoi?

\footnotetext{
${ }^{7}$ Josiane Gagnon, projet Nature et santé.
} 


\section{Fiche de retour}

\section{Activité 8 - Nature et santé 8}

Nom :

Date :

1. Nomme-moi un élément de la nature que tu as :

Vu

Entendu

Senti

Goûté

Touché

2. Décris-moi un moment où tu t'es senti émerveillé, où tu as appris quelque chose :

3. Dis-moi ce que tu as appris sur toi-même au cours de l'activité?

\footnotetext{
${ }^{8}$ Josiane Gagnon, projet Nature et santé.
} 
Fiche de retour

\section{Coucher intérieur - Nature et santé 9}

Nom :

Date:

1. Raconte-moi, dans tes mots, comment tu as vécu l'ensemble de l'activité (relaxation, bouffe, présentation aux parents, coucher à l'école). Quelles émotions as-tu ressenties? Quel(s) moment(s) as-tu préféré(s)? Est-ce que certaines choses t'ont impressionné?

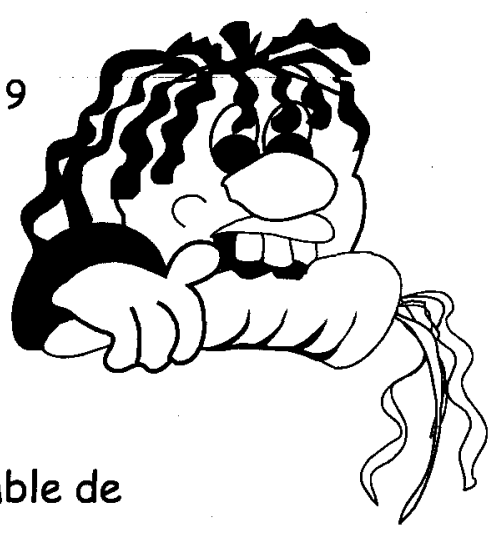$$
\text { preferé(s)? Est-ceque certaines choses t'ont impressionne? }
$$

\footnotetext{
${ }^{9}$ Josiane Gagnon, projet Nature et santé.
} 
Fiche de retour

Lac Emmuraillé - Nature et santé ${ }^{10}$

Nom:

Date :

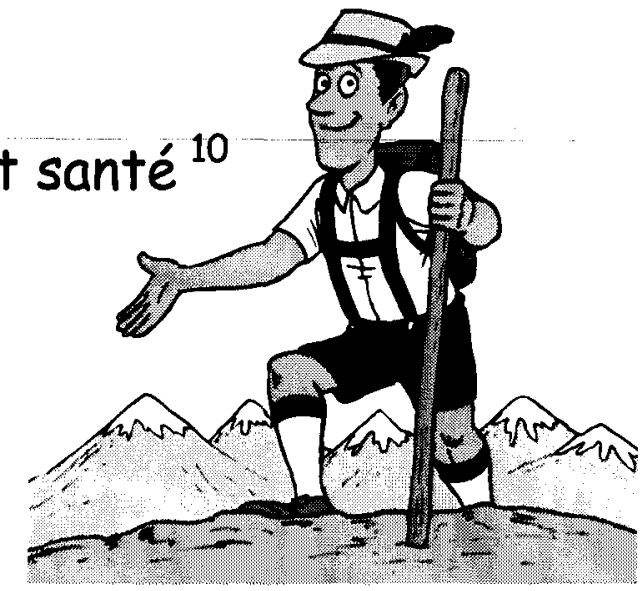

1. Au cours de l'activité tu as vécu différentes situations, décris-moi un moment où tu as pris conscience de ton corps :

2. Nomme-moi deux émotions que tu as ressenties lors de ce moment :

i.

ii.

3. Selon toi quels éléments (froid, vent, exercice, l'euphorie d'être entre amis, le fait d'avoir réalisé quelque chose...), ont pu causer ces émotions?

4. En un seul mot, décris-moi ce que tu as vécu :

En une seule phrase, dis-moi ce que tu as appris sur toi même:

${ }^{10}$ Josiane Gagnon, projet Nature et santé. 


\section{Questionnaire final Nature et santé $e^{11}$ - Jeune}

1. Nomme-moi le sujet choisi (pistes d'animaux, champignons, eau, etc.) lors de la randonnée à la chute :

2. Quel était le nom de tes partenaires :

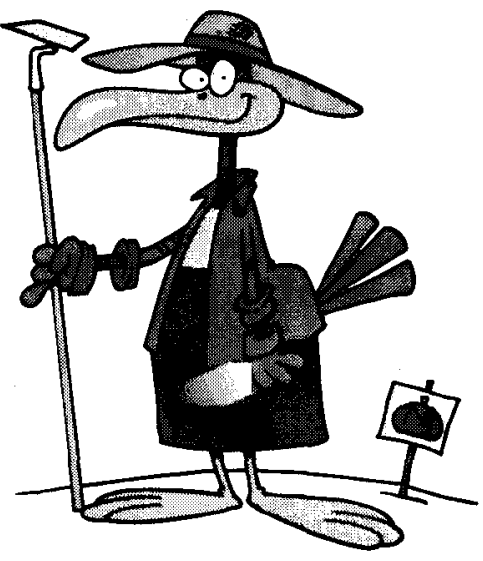

3. Explique-moi pourquoi ton équipe a préféré ce sujet :

4. Quel rôle as-tu joué dans l'élaboration de ton projet? Explique-moi le déroulement :

${ }^{11}$ Josiane Gagnon, projet Nature et santé. 
5. Aujourd'hui qu'est-ce que ce sujet représente pour toi?

\section{De façon générale}

6. Est-ce que le projet Nature et santé t'a permis de découvrir de nouvelles choses à l'école?

Merci beaucoup de ta collaboration! 


\section{Annexe 7}

Canevas d'entrevue pour les titulaires de classe 


\section{Canevas d'entrevue pour les titulaires de classe}

1. Avez-vous effectué des changements au niveau de vos méthodes et stratégies d'enseignement?

$\checkmark \quad$ Dans la façon d'aborder les jeunes;

$\checkmark \quad$ dans la façon de proposer les activités en classe;

$\checkmark \quad$ dans la façon de gérer votre classe ou de présenter la théorie.

2. Avez-vous perçu des changements chez les jeunes dans leur :

$\checkmark$ Développement;

$\checkmark$ relation;

$\checkmark$ comportement;

$\checkmark$ motivation.

3. Avez-vous observé des changements dans la relation parent - enfant?

4. Votre vision de l'école a-t-elle changé?

5. Qu'avez-vous trouvé de plus difficile dans l'implantation d'un tel projet? Par étape...

6. Comment avez-vous intégré les activités vécues dans le cadre du projet dans votre pratique quotidienne de l'enseignement? 


\section{Annexe 8}

Canevas d'entrevue pour l'enseignante d'éducation physique et à la santé 


\title{
Canevas d'entrevue pour l'enseignante Éducation physique et à la santé
}

\author{
Durée : 50 minutes
}

1. Quelle est votre perception par rapport à l'expérience vécue par les jeunes de $5^{\mathrm{e}}$ et $6^{\mathrm{e}}$ année?

2. Toujours en lien avec l'expérience :

$\sqrt{ }$ Avez-vous remarqué des changements au niveau de leur développement?

$\checkmark$ Avez-vous remarqué des changements de comportements chez les jeunes?

$\sqrt{ }$ Avez-vous remarqué des changements dans leurs relations interpersonnelles?

$\sqrt{ }$ Avez-vous remarqué d'autres changements?

3. Croyez-vous qu'une évolution « $\mathrm{x}$ » est attribuable à l'expérience qu'ils ont vécue?

4. Quels impacts, croyez-vous, le projet a-t-il eus sur les jeunes?

5. Croyez-vous que le projet a modifié votre relation avec les enseignantes qui y participaient? 
Annexe 9

Questionnaire pour la direction de l'école Fréchette 


\section{Questionnaire destiné à la direction \\ de l'école Fréchette ${ }^{1}$}

\section{Coordonnées du directeur de l'école Fréchette}

Nom :

Téléphone :

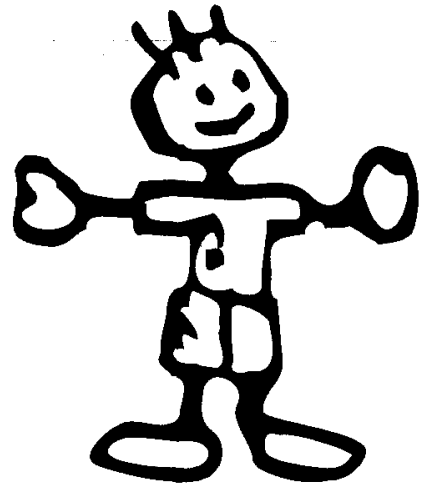

1. Lorsque vous avez entendu parler du projet Nature et santé, quelle a été votre réaction relativement à la mise en place de cette expérience?

2. Maintenant, quelle est votre perception par rapport à l'expérience que les élèves de $5^{\mathrm{e}}$ et $6^{\mathrm{e}}$ année ont vécue?

3. a) Au cours de l'expérience avez-vous ressenti certaines craintes?

b) Comment aurions-nous pu pallier ces craintes? Expliquez :

' Josiane Gagnon, projet Nature et santé. 
4. Au cours de la présente année scolaire, avez-vous perçu chez les enseignantes ayant participé à l'expérience des changements quant à l'utilisation de certaines méthodes ou stratégies pédagogiques?

5. Au cours de la présente année scolaire, avez-vous perçu un changement au niveau des relations interpersonnelles établies entre les enfants ayant participé à l'expérience et leurs enseignantes?

6. Au cours de la présente année scolaire, avez-vous perçu un changement de motivation à l'égard de l'école chez les enfants ayant participé à l'expérience?

7. À votre avis, les activités d'aventure et plein air vécues par les élèves ont-elles eu un impact sur leur développement, sur leur comportement ou sur leurs relations interpersonnelles?

Précisez : 
8. Au cours de l'année scolaire comment avez-vous perçu les réactions des parents par rapport à l'expérience?

9. Selon vous, quelle est la perception des autres enseignants envers le projet Nature et santé?

Enseignants du primaire :

Enseignants du secondaire :

10. Estimez-vous que le projet Nature et santé a eu un impact sur l'ensemble de l'école?

Expliquez: 
11. L'implantation d'un tel projet à la grandeur d'une école requiert un processus d'intégration. Selon vous, quels sont les facteurs favorisant la mise en place d'une approche Aventure et plein air en milieu scolaire? Énumérez dans l'ordre les éléments essentiels à une implantation réussie :

Merci de votre collaboration!

Sachez que je vous informerai des résultats obtenus.

Josiane Gagnon Dt. P. et étudiante à la maîtrise en Éducation, Aventure et plein air 


\section{Annexe 10}

\section{Questionnaire destiné aux parents}


Objet : Questionnaire aux parents (ou tuteurs) concernant l'estime de soi des élèves de $5^{e}$ et $6^{e}$ année du primaire.

Chers parents ou tuteurs,

À la suite des expériences nature et plein air qu'ont vécues les jeunes inscrits en $5^{\mathrm{e}}$ et $6^{\mathrm{e}}$ année du primaire de l'école Fréchette de L'Anse-Saint-Jean, la présente a pour but de vous demander de répondre aux questions ci-jointes pour connaître votre opinion.

Sachez que votre implication nous aidera à améliorer les interventions futures et favorisera un meilleur épanouissement chez les jeunes de notre milieu.

Merci de votre collaboration.

Nadia Rose, enseignante

Julie Bergeron, enseignante

Josiane Gagnon, Intervenante Nature et santé.

Étudiante à la maîtrise à l'UQAC

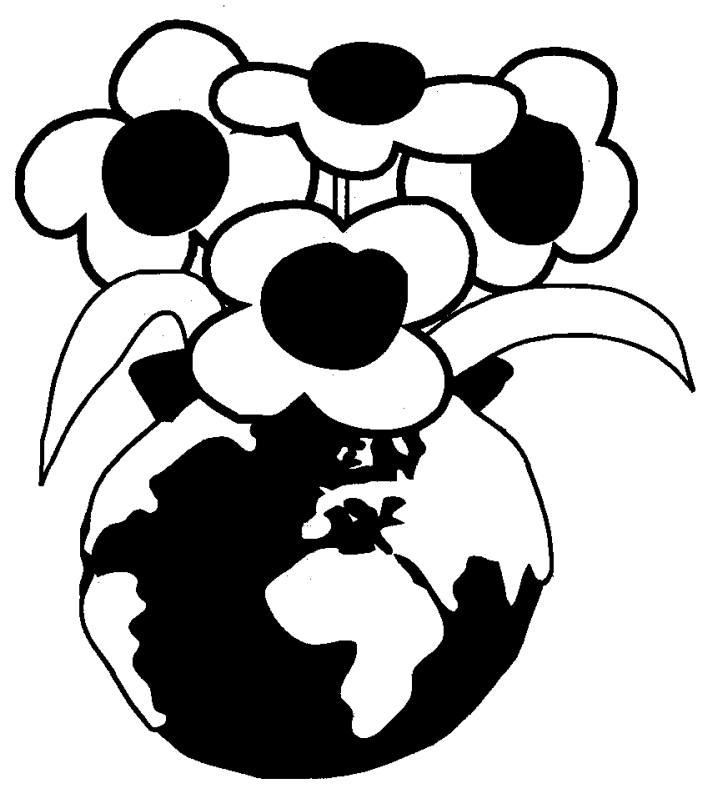

S.V.P. veuillez retourner le questionnaire dûment rempli à l'école 


\section{Questionnaire parents (ou tuteurs) Nature et}

santé

1. La première fois que vous avez entendu parler du projet Nature t santé, quelle a été votre réaction relativement à la mise en place de cette expérience?

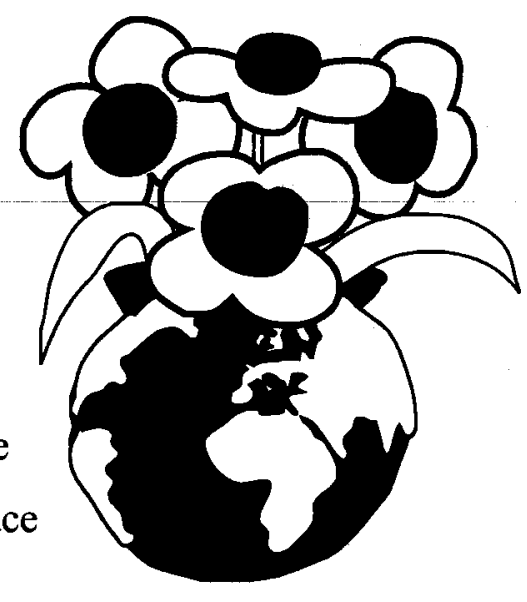

2. À la suite de la participation de votre enfant à ce projet, maintenez-vous toujours la même opinion?

Oui

Non

Expliquez :

3. Au cours de l'expérience avez-vous ressenti des craintes?

4. Y a-t-il des informations pertinentes que vous auriez aimé savoir avant et au cours des activités?

${ }^{2}$ Josiane Gagnon, projet Nature et santé. 
5. Au cours de la présente année scolaire, avez-vous perçu un changement au niveau de la relation que vous entretenez avec votre enfant?

6. À votre avis, les activités en plein air qu'a vécues votre enfant ont-elles eu un impact sur son développement, sur son comportement ou sur ses relations interpersonnelles? Si oui, précisez.

Impact sur son développement :

Impact sur son comportement :

Impact sur ses relations interpersonnelles (amis(es), frère(s) et saur(s), parent(s)):

7. Si l'expérience était reconduite (refaite) quelles recommandations auriez-vous à nous faire? 


\section{Annexe 11}

Tableaux synthèse des instruments de mesures Compétences transversales d'ordre personnel et social

Légende :

$$
\begin{aligned}
& A=\text { Ateliers (Al à A10); } \\
& Q=\text { Questions posées (fiche de retour ou retour oral); } \\
& S=\text { Sujet. }
\end{aligned}
$$




\title{
COMPÉTENCES TRANSVERSALES D'ORDRE PERSONNEL ET SOCIAL
}

\author{
Compétence transversale 7 : Structurer son identité
}

Tableau A : S'ouvrir aux stimulations environnantes

\begin{abstract}
Extraits du journal de bord - Commentaires de
la chercheure-acteure et propos des

enseignantes lors des entrevues finales

\section{Extrait du journal de bord - A1}

Les jeunes ont senti, touché, écouté, goûté et entendu des choses, ils ont exploré avec leurs cinq sens, leur trésor.
\end{abstract}

Extraits du journal de bord - A8

"Touchez-le! Goûtez-le! Sentez-le! », voilà la consigne que les jeunes avaient afin d'identifier leur arbre ami. En deuxième étape, ils devaient, les yeux bandés, retrouver l'arbre en question. Les jeunes ont semblé apprécier cette activité. Certains m'ont dit qu'après l'avoir bien senti et palpé, l'arbre était facile à trouver et ce, même les yeux fermés.

Un éveil auditif et gustatif: (Les bruits) Ils sont loin! Ils sont proches! Ils sont forts! Ils sont sourds! Cherchez à comprendre d'où ils proviennent. Identifiez-moi un bruit près de vous...je laisse écouler un peu de temps...Identifiez-en un loin de vous.... Un très sourd... grave. [...] En deuxième partie, les yeux fermés, les jeunes sont invités à goûter les fruits du vinaigrier. La consigne : "Identifiez les sensations gustatives perçues; acide, sucré, rugueux... ». Tous ont bien participé, ils étaient concentrés et attentifs!

Extrait du journal de bord - A9

Les jeunes sont craintifs de goûter certains mets ou aliments, mais ils doivent tout de même y goûter par respect pour les cuisiniers, pour la découverte et l'ouverture sur le monde.
A2-Q1 : Selon toi, quel(s) lien(s) as-tu avec la nature? S1: Il y a des bruits d'animaux et de l'eau qui coule Act8-Q2 : Décris-moi un moment où tu t'es senti émerveillé et où tu as appris quelque chose :

S21 : [...] J'étais seul et concentré par le bruit alentour, $j$ 'étais bien.

S22 : Quand avec l'activité de Intervenant 5, Il fallait aller dans le bois et entendre les bruits aigus, graves, etc. Là, $j$ 'ai vraiment compris que quand on est dans la forêt, il y a beaucoup de bruits très différents que quand par exemple quelqu'un qui habite près d'une autoroute

A10-Q1 : Au cours de l'activité, tu as vécu différentes situations. Décris-moi un moment où tu as pris conscience de ton corps : S7 : Quand je me suis couchée, j'étais contente de ma très belle journée avec toutes les activités et aussi très excitée de savoir ce qu'on va faire demain en plus j'étais avec mes amies et je m'amusais énormément.

S15: Quand j'ai marché en raquette, pour me rendre au Lac Emmuraillé, j'étais fatiguée, mais en même temps heureuse. S17 : J'étais heureuse d'être arrivée, mais aussi un peu fatiguée. S18: Moi, quand je suis rentré dans le chalet pour me coucher, j'ai ressenti la chaleur du feu se rendre sur mon corps. J'étais très bien.

A10 -Q2 Nomme-moi deux émotions que tu as ressenties lors de ce moment :

S8: L'épuisement

S12: L'intrigue

S20: L'émerveillement

S21: L'impatience

S24: La fierté

S28: La plénitude

A10-Q3 : Selon toi quels éléments (froid, vent, exercice, l'euphorie d'être entre amis, le fait d'avoir réalisé quelque chose...), ont pu causer ces émotions.

$S 17$ : Etre avec mes amies et vivre quelque chose que je n'aurais peut-être pas vécu un jour.

S18: La chaleur du feu qui me réchauffait et de coucher à l'extérieur de chez nous avec nos amis. 
Tableau B : Prendre conscience de sa place parmi les autres

\begin{tabular}{|c|c|}
\hline $\begin{array}{l}\text { Extraits du journal de bord - } \\
\text { Commentaires de la chercheure- } \\
\text { acteure et propos des enseignantes lors } \\
\text { des entrevues finales }\end{array}$ & Citations (ou écrits) des jeunes \\
\hline $\begin{array}{l}\text { Enseignante d'éducation } \\
\text { physique et à la santé } \\
\text { [...] lui j'peux dire que ça été super } \\
\text { bon [...] même (au niveau de) sa } \\
\text { relation avec les autres. T'sais au } \\
\text { départ les autres avaient tendance à } \\
\text { pas trop le vouloir dans leur équipe, } \\
\text { mais à la fin, y'était intégré... ouais. } \\
\text { Extraits du journal de bord } \\
\text { A2 } \\
\text { L'enseignante } 2 \text { trouve très } \\
\text { intéressant de voir évoluer les jeunes } \\
\text { dans un autre contexte. Cet atelier } \\
\text { m'a permis de voir les leaders se } \\
\text { démarquer } \\
\text { A5 } \\
\text { Selon l'enseignante 2, l'activité a } \\
\text { permis de démontrer aux jeunes que } \\
\text { personne ne possède la vérité et que } \\
\text { tout le monde peu se tromper, il faut } \\
\text { apprendre à s'adapter et à dépasser } \\
\text { ses limites }\end{array}$ & $\begin{array}{l}\text { A7-Q2 : As-tu trouvé facile ou difficile de faire confiance aux } \\
\text { autres? } \\
\text { S2: Ce sont mes ami(e)s et je leur fais confiance...c'est comme } \\
\text { mes frères et mes saurs. } \\
\text { S15 : Je savais qu'il ne me laisserait jamais tomber, alors je leur } \\
\text { faisais confiance. } \\
\text { A8-Q3 : Dis-moi ce que tu as appris sur toi-même au cours de } \\
\text { l'activité? } \\
\text { S17: J'ai appris que j'étais capable de me faire confiance. } \\
\text { S29: Que je peux être sérieux. } \\
\text { A10-Q3 : Selon toi, quels éléments (froid, vent, exercice, } \\
\text { l'euphorie d'être entre amis, le fait d'avoir réalisé quelque } \\
\text { chose, etc.), ont pu causer ces émotions. } \\
\text { S10: L'euphorie d'être entre ami(e)s. }\end{array}$ \\
\hline \multicolumn{2}{|c|}{$\begin{array}{l}\text { Commentaires de titulaires de classe - inscrits dans le journal de bord à la suite du retour à } \\
\text { mi-parcours (A6) } \\
\text { J'ai apprécié voir évoluer les jeunes dans un autre contexte que la classe. J'ai appris à } \\
\text { découvrir l'enseignement sous un autre angle (enseignante 2). } \\
\text { J'ai appris que les comportements en classe cachaient la personnalité de jeunes (enseignante 1 }\end{array}$} \\
\hline
\end{tabular}


Tableau $\mathrm{C}$ : Mettre à profit ses ressources personnelles

Extraits du journal de bord - Commentaires de la chercheure-acteure et propos des enseignantes lors des entrevues finales

Extraits du journal de bord

$\mathbf{A 3}$

Les jeunes habitent leur corps utilisent leur potentiel corporel ainsi que l'ensemble de leurs compétences pour parvenir à leurs fins.

[...] Respiration, jeux d'habiletés physiques et exercices de concentration jumelés à des activités de créativité (utilisant leur imagination) sont des éléments qui permettent aux jeunes de mieux se connaitre et de s'apprécier davantage comme des êtres uniques.

\section{Enseignante d'éducation physique et à la santé}

La première sortie (A4 - randonnée à la chute). Ben, j'vois S5. (le défi) était pas si gros, mais pour lui ça devait être gros, pis tu vois y'a été capable de faire la dernière sortie (qui était un plus gros défi), le coucher en forêt [...] t'sais ça y'a permis de voir...oui c'est difficile, mais : " hop! J'ai passé au travers, je suis capable d'évoluer là-dedans ».

S15 T'sais son attitude, j'pense qu'elle était souvent, pas très ouverte à ce qu'on faisait (avant)... pas très souriante aussi [...] Finalement, là-dedans (la séquence d'aventure et d'apprentissage), elle s'est ouverte. Elle a enlevé son masque, parce que t'sais, elle est bourrée de potentiel. Elle a du potentiel, pis elle est capable! Capable de tout faire ce qu'il y'a à faire

\section{Enseignante 2}

$J$ 'pense que quand y (S5) va arriver devant une autre difficulté, il va penser à ça pis ça va l'aider à relever le défi finalement De l'autonomie, je pense plus de responsabilité, de la débrouillardise [...].

Relever des défis, se dépasser. Pour certains aussi au niveau de l'organisation.

Oui y'a eu des grains, pis oui, ils vont y penser face à une autre difficulté et c'est plusieurs projets comme ça qui vont amener un enfant à ce développer

Les jeunes avaient du plaisir [...] y'étaient fiers d'eux-mêmes, de montrer à leurs parents que c'est eux qui avaient "popoté ".

\section{Enseignante 1}

... l'autonomie s'est développé... les compétences... manuelles... d'organisation; l'échange avec les parents. Par exemple, la cuisine c'était vraiment l'fun d'les voir.
Citations (ou écrits) des jeunes

A8-Q3 : Dis-moi ce que tu as appris sur toi-même au cours de l'activité

S9: Je ne me croyais pas capable de surmonter 5 kilomètres.

S10 : Je suis capable de durer longtemps.

S11 : Je pouvais marcher une longue distance, je ne sais pas que j'étais capable.

S13 : J'étais capable de marcher aussi longtemps.

S21 : J'ai appris (...) que si je veux, je peux.

A10-Q3 : Selon toi, quels éléments (froid, vent, exercice, l'euphorie d'être entre amis, le fait d'avoir réalisé quelque chose, etc.), ont pu causer ces émotions. $S 1: Q$ 'on est plein d'énergie. S4: J'ai appris que j'étais capable de transporter un gros sac sur le dos.

S5: J'ai appris à avoir confiance en moi.

S7 : Je suis capable de faire des défis.

S8 : Quej'étais capable de marcher 4 kilomètres.

S9: Quej'étais capable de relever des défis.

S10 : Que j'étais capable de marcher loin.

S12: Que je pouvais marcher longtemps de même. [...] si je veux, je peux!

S13: Quej'avais de l'imagination. S16:J'ai appris que j'étais capable de transporter mon sac à dos pendant une grande distance. S18: Que j'ai relevé le défi de marcher avec un gros sac sur le dos. 


\section{Compétence transversale 8 : Coopérer}

Tableau D : Interagir avec ouverture d'esprit dans différents contextes

\begin{tabular}{|c|c|c|}
\hline $\begin{array}{l}\text { Extrait du journal de bord - } \\
\text { Commentaires de la chercheure- } \\
\text { acteure }\end{array}$ & $\begin{array}{l}\text { Propos des enseignantes lors des } \\
\text { entrevues finales }\end{array}$ & Citations (ou écrits) des jeunes \\
\hline $\begin{array}{l}\text { A2 } \\
\text { [...JJe souhaite que cet exercice ait } \\
\text { permis à plusieurs jeunes de } \\
\text { comprendre ou de visualiser, qu'il } \\
\text { existe plusieurs liens entre les } \\
\text { organismes vivant dans la nature. } \\
\text { [... Que tous doivent collaborer, } \\
\text { afin de se développer et de vivre } \\
\text { (survivre). L'exercice a certainement } \\
\text { permis à quelques-uns de cheminer } \\
\text { sur les connaissances vues en classe. } \\
\text { J'ai hâte de voir s'ils feront des liens } \\
\text { avec leur survie! } \\
\text { A3 } \\
\text { [...] j'ai remarqué que les jeunes } \\
\text { travaillaient de façon individuelle... } \\
\text { Je constate que la coopération et les } \\
\text { forces de chacun ne sont pas } \\
\text { exploitées au maximum, l'esprit } \\
\text { d'équipe est donc à développer. Les } \\
\text { jeunes sont individualistes. } \\
\text { A6 } \\
\text { Défi demandant beaucoup } \\
\text { d'entraide et de collaboration : Je } \\
\text { sens beaucoup d'enthousiasme de la } \\
\text { part des jeunes, ils sont tous présents } \\
\text { et très attentifs. «Intervenant } 5, \text { On } \\
\text { veut le refaire! ". " d'accord, une } \\
\text { autre fois ». Plus habiles dans leurs } \\
\text { mouvements et encore plus attentifs, } \\
\text { les jeunes s'en donnent à coeur joie } \\
\text { [...] Les enseignantes l'essaient } \\
\text { aussi. Je sens une belle frénésie! }\end{array}$ & $\begin{array}{l}\text { Enseignante d'éducation physique } \\
\text { et à la santé } \\
\text { J'pense que c'est plus, justement, } \\
\text { au niveau de la coopération et de } \\
\text { l'entraide. J'ai pas vu personne } \\
\text { d'isolée. Même au contraire, quand } \\
\text { y'en avait un (ou une), pour qui } \\
\text { c'était plus difficile,... (y'avait) tout } \\
\text { le temps quelqu'un pour l'aider. } \\
\text { (..) } \\
\text { je te disais tantôt, ce qui a été l'fun, } \\
\text { c'est vraiment qu'ils se sont mixés } \\
\text { et qu'on sent jamais qu'y'ait } \\
\text { d'élèves rejets. } \\
\text { Enseignante } 2 \\
\text { Beaucoup de motivation parce que } \\
\text { c'était lié directement au projet } \\
\text { (éducation par l'aventure). Le choix } \\
\text { s'était fait en sortie, pis dans les } \\
\text { autres sorties, ils accumulaient des } \\
\text { informations sans compter les } \\
\text { informations recueillies dans des } \\
\text { livres et sur le web qui } \\
\text { contribuaient à enrichir leur projet. }\end{array}$ & $\begin{array}{l}\text { A2 -Q3 Crois-tu important de } \\
\text { collaborer avec les autres? } \\
\text { S4 : Parce que si on ne collabore pas } \\
\text { avec les amis, ils ne joueront plus } \\
\text { avec nous. } \\
\text { S6: On peut échanger des idées. } \\
\text { S9: Les uns ont besoin des autres } \\
\text { pour vivre. } \\
\text { S11: Il faut collaborer pour réussir } \\
\text { quelque chose qu'on n'est pas } \\
\text { capable de faire tout seul. } \\
\text { S13: Parce que c'est important de } \\
\text { s'entraider. } \\
\text { S15: Pour la survie de l'humain, } \\
\text { pour vivre et pour grandir. Pour } \\
\text { l'épanouissement, pour les } \\
\text { expériences de la vie! } \\
\text { S16: Parce que si on ne collaborait } \\
\text { pas on aurait pas d'amis (es) et ce } \\
\text { serait difficiles d'apprendre. } \\
\text { S17: Pour s'aider et demander de } \\
\text { l'aide. Donner et demander des } \\
\text { opinions aux autres. } \\
\text { S20: Parce que parfois, il faut } \\
\text { s'entraider, on ne réussirait pas notre } \\
\text { vie sans les autres. } \\
\text { S23 Parce que ça garde notre bonne } \\
\text { humeur. }\end{array}$ \\
\hline \multicolumn{3}{|c|}{$\begin{array}{l}\text { Commentaires des parents (questionnaire final) } \\
\text { S15:[...] amélioration de la complicité avec ses amis (es). } \\
\text { S21:[...] plus réceptive face aux idées des autres tout en sachant exprimer ses idées de façon respectueuse. } \\
\text { S24: Il a évolué dans sa relation avec ses pairs. }\end{array}$} \\
\hline
\end{tabular}


Tableau E : Contribuer au travail collectif

\begin{tabular}{|c|c|c|}
\hline $\begin{array}{l}\text { Extrait du journal de bord - } \\
\text { Commentaires de la chercheure- } \\
\text { acteure }\end{array}$ & $\begin{array}{l}\text { Propos des enseignantes, entrevues } \\
\text { finales }\end{array}$ & Citations (ou écrits) des jeunes \\
\hline $\begin{array}{l}\text { A1 } \\
\text { Pour arriver à découvrir la } \\
\text { boîte cachée, ils devaient } \\
\text { travailler en équipe, une } \\
\text { équipe a tardé à trouver le } \\
\text { premier azimut, car ces } \\
\text { derniers essayaient de } \\
\text { résoudre le problème de } \\
\text { façon individuelle. } \\
\text { Lorsqu'ils se sont décidés à } \\
\text { travailler en équipe, ils ont } \\
\text { rapidement trouvé le trésor } \\
\text { caché. (extrait du journal } \\
\text { de bord) } \\
\text { A2 } \\
\text { Les titulaires de classe ont noté } \\
\text { au cours de l'atelier l'émergence } \\
\text { de manifestations reliés à la } \\
\text { composante: 'contribuer au } \\
\text { travail collectif". l'enseignante } 2 \\
\text { a ajouté qu'elle trouvait } \\
\text { intéressant de voir évoluer les } \\
\text { jeunes dans un autre contexte. } \\
\text { l...] cet atelier m'a permis de } \\
\text { voir de nouveaux leaders se } \\
\text { démarquer [...] et à l'élaboration } \\
\text { de stratégies de communication. }\end{array}$ & $\begin{array}{l}\text { Enseignante d'éducation physique et à } \\
\text { la santé : } \\
\text { T'sais sa relation avec les autres... Au } \\
\text { début, c'était pas facile. Il était pas } \\
\text { motivé. Il s'impliquait plus ou moins. Il se } \\
\text { démarquait par sa "non-présence" } \\
\text { (manque d'implication). Pis à la fin, il } \\
\text { passait comme les autres, pis t'sais, y } \\
\text { jouait comme les autres, pis y s'est } \\
\text { intégré au fond. [...] } \\
\text { Enseignante } 1 \\
\text {... t'sais (S5), il était fier de lui, ben y'en } \\
\text { a d'autres qui étaient fiers de lui aussi. } \\
\text { Quand il a réussi, il s'est fait féliciter } \\
\text { (référence à A4). } \\
\\
\text { Enseignante } 2 \\
\text { [...] ceux qui ont entraidé, ils vont } \\
\text { continuer à s'entraider. C'est démarré. } \\
\text { Ceux qui ont encouragé, pis qui } \\
\text { n'encourageaient pas y'vont... t'sais, ils } \\
\text { l'on vécu... ça va se poursuivre! }\end{array}$ & $\begin{array}{l}\text { A10-Q4 :En un seul mot décris- } \\
\text { moi ce que tu as vécu : } \\
S 2: \text { Beaucoup d'entraide } \\
S 11 \text { : Entraide } \\
S 22: \text { Entraide } \\
\text { Retour à mi-parcours : } \\
\text { Ce que j'ai appris: } \\
S 5: \text { A travailler avec les autres. }\end{array}$ \\
\hline
\end{tabular}


Tableau F : Tirer profit du travail en coopération

\begin{tabular}{|c|c|c|}
\hline $\begin{array}{l}\text { Extrait du journal de bord - } \\
\text { Commentaires de la chercheure- } \\
\text { acteure }\end{array}$ & $\begin{array}{l}\text { Propos des enseignantes, entrevues } \\
\text { finales }\end{array}$ & Citations (ou écrits)) des jeunes \\
\hline $\begin{array}{l}\text { A2 } \\
\text { Passe la passe, cette activité } \\
\text { demandait beaucoup d'entraide et } \\
\text { d'écoute de la part des } \\
\text { partenaires d'équipe. Par } \\
\text { exemple, S24 n'avait pas eu de } \\
\text { passe et } S 2 \text { (l'un des membres de } \\
\text { son équipe) ne l'a pas cru. } \\
\text { Résultat l'équipe a perdu faute } \\
\text { d'avoir réussi à passer le ballon à } \\
\text { chaque membre de l'équipe. } \\
\text { l...J Les jeunes ont perçu lors de } \\
\text { ces activités l'essentiel du travail } \\
\text { d'équipe et de la collaboration. } \\
\text { Ils ont pu comprendre et } \\
\text { visualiser qu'il existe plusieurs } \\
\text { liens entre les organismes vivant } \\
\text { dans la nature et que tous doivent } \\
\text { collaborer afin de vivre et de se } \\
\text { développer. }\end{array}$ & $\begin{array}{l}\text { Enseignante } 2 \\
\text { J'ai vu naître de l'entraide. } \\
\text { Enseignante } 1 \\
\text { [...] de l'entraide et de la } \\
\text { confiance en soi, c'est des grains } \\
\text { qui ont été faits. } \\
\\
\text { Le fait d'encourager les autres. } \\
\text { T'sais même si quelqu'un a plus } \\
\text { de difficulté... ben t'sais... on } \\
\text { lâche pas. } \\
\text { Suppléante } \\
\text { Je trouvais ça drôle (bien) quand } \\
\text { les jeunes allaient s'aider les } \\
\text { autres à arranger leur sac. c'était } \\
\text { bon! } \\
\text { Enseignante d'éducation } \\
\text { physique } \\
\text { C'est ça au fur et à mesure, } \\
\text { j'pense qu'ils se sont en allés } \\
\text { vraiment comme ça... Ça (la } \\
\text { séquence d'aventure et } \\
\text { d'apprentissage) construit de } \\
\text { meilleurs liens [...] plus de } \\
\text { coopération. Ça forme un groupe } \\
\text { plus homogène... ouais! C'est ça! }\end{array}$ & $\begin{array}{l}\text { Retour à mi-parcours - oral } \\
\text { J'ai appris que les autres sont } \\
\text { différents et ils peuvent nous } \\
\text { aider. } \\
\text { A10 - Q3 : selon toi quels } \\
\text { éléments, ont pu causer ces } \\
\text { émotions } \\
\text { S1 : c'était froid et on a lutté } \\
\text { tous ensemble. }\end{array}$ \\
\hline
\end{tabular}




\section{Annexe 12}

Demande d'intervenants stagiaires en plein air et touriste d'aventure de l'UQAC 
L'Anse St-Jean, le 3 Janvier 2004

Mario Bilodeau

Professeur Plein air et Tourisme d'aventure

Département des sciences humaines

555 boulevard de l'Université

Chicoutimi, (Québec) Canada

G7H 2B1

Objet : Demande d'intervenants en plein air

Monsieur Bilodeau,

Nous sommes présentement, à mettre en place à l'école primaire et secondaire Fréchette de l'Anse St-Jean, dans la région du Bas-Saguenay, un programme Nature. Ce programme comporte plusieurs volets dont un volet plein air (activités d'aventure). Pour arriver à bien ficeler le projet et intégrer aux activités éducatives, différentes activités de plein air et d'aventure nous avons besoin d'intervenants compétents pour créer, animer et encadrer ce genre d'activités.

Il est à noter que les activités mises en place, en plus de rejoindre les objectifs du Programme de formation de l'école québécoise, auront pour but de rehausser l'estime de soi des jeunes et de développer chez eux des comportements responsables face à leur santé (alimentation, activité physique...) et leur environnement. Une étude universitaire est présentement à évaluer ces composantes.

C'est afin de trouver des intervenants compétents que nous nous adressons à vous. Merci à l'avance d'aider les jeunes de notre région à vivre un contact privilégié avec la nature qui les entoure.

Jean-François Lanoue, coordonnateur du projet éducatif de l'école Fréchette

Josiane Gagnon, Dt.P. Intervenante en promotion de la santé et étudiante à la Maîtrise à en éducation plein air. 